\title{
Using Imperfect Semiconductor Systems for Unique Identification
}

\author{
Jonathan David Roberts
}

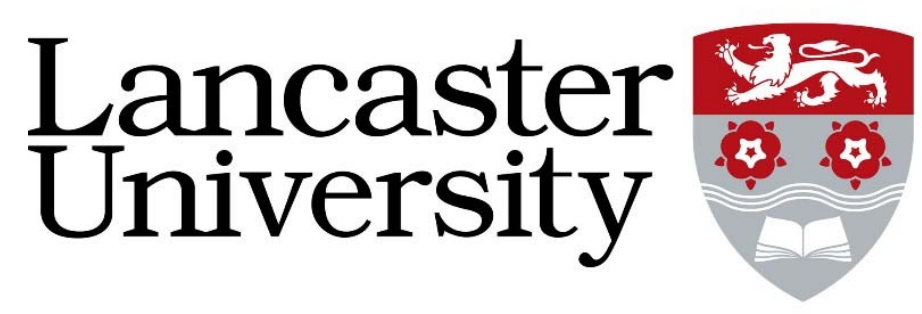

Department of Physics

Lancaster University

September 2016

This thesis is submitted in partial fulfilment of the requirements for the degree of Doctor of Philosophy 


\section{Declaration}

This thesis is the result of work carried out at Lancaster University between April 2013 and September 2016. I declare that the work presented was carried out by myself and contains nothing which is the outcome of work done in association with others, except as specified in the text. The thesis is not substantially the same as any I have already submitted, or that I am in the process of submitting, for any degree at any university. This thesis does not exceed 80,000 words.

J. D. Roberts, Lancaster, 2016. 


\section{Acknowledgements}

I would first and foremost like to thank my supervisor, Robert Young, for his excellent supervision and invaluable help and guidance. Your tenacity and passion for research should be an example to us all. Thanks for allowing me to take part in this project, it seems a very long time ago since we met in that crowded poster session in Manchester! Thanks for all the interesting projects offered and for the freedom I was given during my studies. You have helped define the researcher I am today and I do not doubt that without you, this project would not have been possible.

For introducing me to the Langmuir-Blodgett suite at Lancaster, Benjamin Robinson deserves a special mention. Without the interesting discussions we had, a lot of this work would never have happened. Thanks for all your help along the way.

Special thanks go out to Mo Missous, Mohamed Zawawi, James Sexton and Max Migliorato for providing the Resonant Tunneling Diode samples for electrical characterisation. Likewise, thanks to Maria Acebrón and Beatriz Juárez for providing the nanoparticle samples. These measurements would have been challenging without your help. Thanks guys.

I've been lucky enough to have the opportunity to work with a large number of people during my time at Lancaster; Chris, Yas, Matt, Yameng, Ramon, Andres, Peter, Phil, Alex, Utz, Ethem. Our fruitful intellectual discussions often provided moments of clarity on what to try next, in some cases anyway! Between you all, you've made my time at Lancaster extremely enjoyable, and I go away from this experience with many unforgettable memories, thanks.

To the guys on my DTC cohort, you made the first six months in Manchester fantastic, so thank you.

To say that I feel profound gratitude to my friends, family and especially Phoebe, my partner, would be a massive understatement. Any attempt to express my appreciation for your unwavering support would be incomplete. I love you all.

Compiling 3 years of your life into one document seems like a daunting task at the beginning, and for allowing me to clear my head and keep my sanity in times of need. I thank my little doggy Douglas.

Finally, I extend gratitude to the EPSRC NoWNano Doctoral Training Centre, for funding me throughout my amazing journey. 


\section{List of Publications}

1. J. Roberts, A. Black, B. J. Robinson, P. Tovee, C. S. Woodhead, Y. J. Noori, Y. Cao, R. Bernado-Gavito, and R. J. Young, "Graphene/Nanoparticle/Graphene Heterostructures Fabricated with the Langmuir-Blodgett Technique" (Manuscript in preparation, 2016).

2. J. Roberts, B. J. Robinson, P. Tovee, C. S. Woodhead, Y. J. Noori, Y. Cao, R. Bernado-Gavito, and R. J. Young, "Deposition of $\mathrm{MoS}_{2}$ Nanosheets Using the Langmuir-Blodgett Technique" (Manuscript in preparation, 2016).

3. R. Bernado-Gavito, J. Roberts, J. Sexton, B. Astbury, H. Shokeir, T. McGrath, Y. J. Noori, C. S. Woodhead, M. Missous, U. Roedig \& R. J. Young, "N-State Random Switching Using Quantum Confinement in Diodes" (under review, Nature Communications, 2016).

4. J. Roberts, R. Bernado-Gavito and R. J. Young, "Generating a Nondeterministic Response to a Challenge", GB1613002.3 (2016).

5. J. Roberts, R. Bernado-Gavito and R. J. Young, "Generating a Unique Response to a Challenge", GB1613002.3 (2016).

6. C. S. Woodhead, J. Roberts, Y. J. Noori, Y. Cao, R. Bernado-Gavito, P. Tovee, A. Kozikov, K. Novoselov and R. J. Young, "Increasing the Light Extraction and Longevity of TMDC Monolayers Using Liquid Formed Micro-lenses", 2D Materials, 4, 1 (2016).

7. Y. J. Noori, Y. Cao, J. Roberts, C. S. Woodhead, R. Bernado-Gavito, P. Tovee and R. J. Young, "Photonic Crystals for Enhanced Light Extraction from 2D Materials", ACS Photonics, Article ASAP.

8. J. Roberts, U. Roedig and R. J. Young, "Quantum Physical Unclonable Function", GB1611554.5 (2016).

9. J. Roberts, I. E. Bagci, M. A. M. Zawawi, J. Sexton, N. Hulbert, Y. J. Noori, M. P. Young, C. S. Woodhead, M. Missous, M. A. Migliorato, U. Roedig and R. J. Young, “Atomic-scale Authentication with Resonant Tunneling Diodes", MRS Advances, 5 (2016).

10. J. Roberts, P. Speed and R. J. Young, "Using Quantum Effects in Nanomaterials for Unique Identification", SPIE Newsroom, 3 (2016). 
11. J. Roberts, I. E. Bagci, M. A. M. Zawawi, J. Sexton, N. Hulbert, Y. J. Noori, M. P. Young, C. S. Woodhead, M. Missous, M. A. Migliorato, U. Roedig and R. J. Young, "Using Quantum Confinement to Uniquely Identify Devices", Scientific Reports, 16456 (2015).

12. J. Roberts, U. Roedig and R. J. Young, "Unique Identifier”, GB1406002.4 (2014).

13. M. P. Young, C. S. Woodhead, J. Roberts, Y. J. Noori, M. T. Noble, A. Krier, E. P. Smakman, P. M. Koenraad, M. Hayne and R. J. Young, "Photoluminescence Studies of Individual and Few GaSb/GaAs Quantum Rings", AIP Advances, 4, 117127 (2014).

14. R. A. Griffiths, A. Williams, C. Oakland, J. Roberts, A. Vijayaraghavan and T. Thomson, "Directed Self-assembly of Block Copolymers for use in Bit Patterned Media Fabrication", Journal of Physics D, 46, 503001 (2013). 


\section{Summary}

The secure identification of an object or electronic system is carried out through the provision of some unique internal or external characteristic. The most obvious examples of these include passwords and fingerprints that can identify a person or an electronic device, and holograms that can tag any given object to provide a check of its authenticity. Unfortunately, modern technology provides resources that enable the trust of these everyday techniques to be undermined.

Identification schemes have been proposed to address these issues by extracting the identity of a system from its underlying physical structure, which is constructed such that the system is hard-toclone or predict. These systems are known as Unique Objects (UNOs) and Physically Unclonable Functions (PUFs).

The aim of the work in this thesis is to create a novel type of UNO/PUF that utilises the atomic-scale uniqueness of semiconductor devices by measuring a macroscopic quantum property of the system. The variations in these quantum properties are amplified by the existence of such atomic-scale imperfections, meaning these devices would be the hardest possible system to clone, use the least resources and provide robust security. Such devices would be of great societal and political significance and would provide the biggest technological barrier between the good guys and the bad. Specifically, this work has introduced three distinct devices based on semiconducting systems that could provide atomic-scale unique identification:

- Electronically - Fluctuations in the current-voltage characteristics of Resonant Tunneling Diodes (RTDs) were found to provide a simple measurement of the underlying quantum state electronically.

- Optically - Macroscopic thin films of the two-dimensional material, $\mathrm{MoS}_{2}$, were created by the Langmuir-Blodgett technique for the first time and have laid the foundations for the formation of an optical analogue of an atomic-PUF/UNO system.

- Optoelectronically - The Langmuir-Blodgett technique's flexibility was utilised to fabricate complex heterostructures that couple graphene to semiconducting nanoparticles. This system should provide an ideal system with efficient electronic and optical characteristics that would be useful in a range of applications, including unique identification. 


\section{Table of Contents}

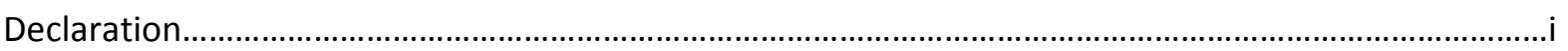

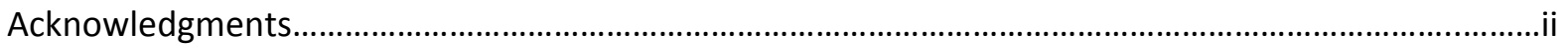

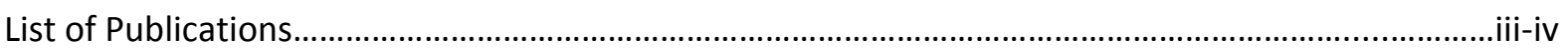

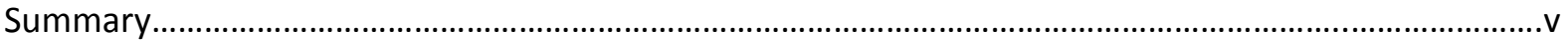

\section{Chapter 1: An Introduction to Security Based on Physical Disorder}

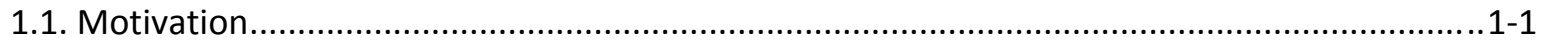

1.2 The Need for a New Era of Authenticity ..................................................................

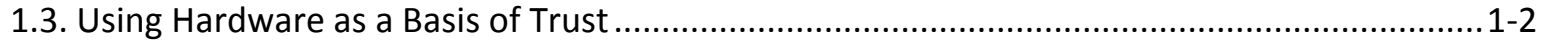

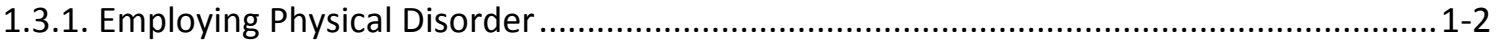

1.3.2. Physically Unclonable Functions ................................................................... 1-3

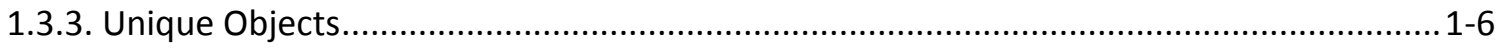

1.3.4. Physical Disorder at the Extreme ................................................................

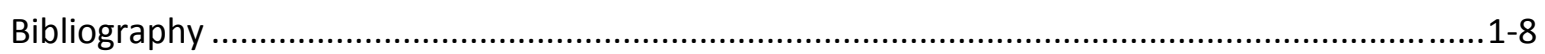

\section{Chapter 2: An Introduction to Semiconductors and Quantum Confinement}

2.1. Introduction ................................................................................................

2.2. Semiconducting Materials ....................................................................................

2.2.1. The Basics ................................................................................................. $2-1$

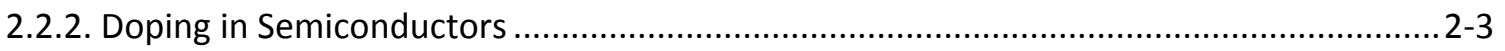

2.2.3. Semiconductor Band Structure ................................................................... 2-5

2.2.4. Direct vs. Indirect Semiconductors ................................................................. $2-8$

2.2.5. Compound Semiconductors...............................................................................

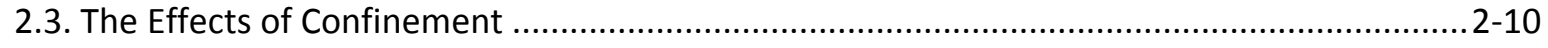

2.3.1. The 'Particle in a Box' Approximation............................................................. 2-10

2.3.2. Beyond 1 Dimension of Confinement ............................................................. $2-13$

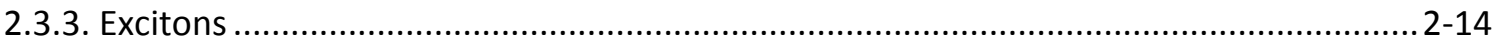

2.4. Aim of This Work: The Sensitivity to Imperfections...................................................2-15

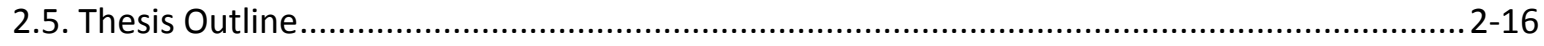

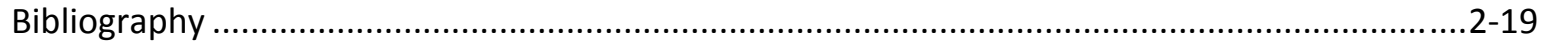




\section{Chapter 3: Sample Preparation and Experimental Techniques}

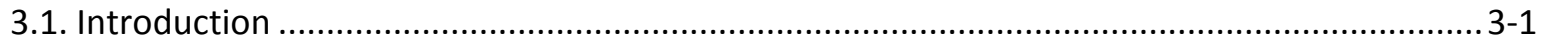

3.2. Production of Resonant Tunneling Diodes ......................................................................

3.2.1. Molecular Beam Epitaxy Growth .................................................................................

3.2.2. Device Fabrication ......................................................................................................

3.2.3. Probe Station Measurements .......................................................................................

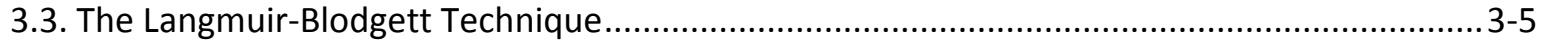

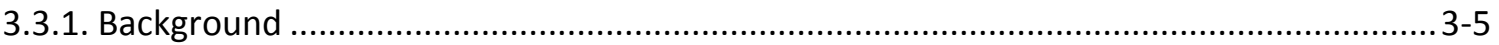

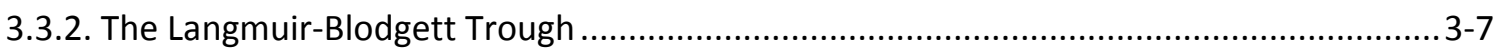

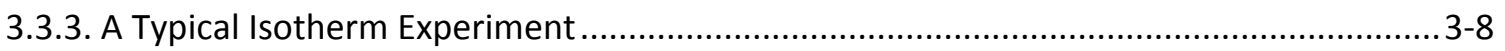

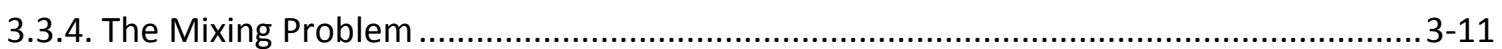

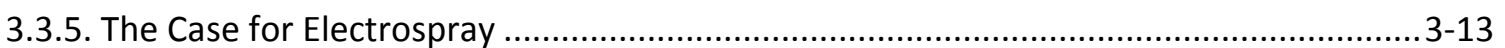

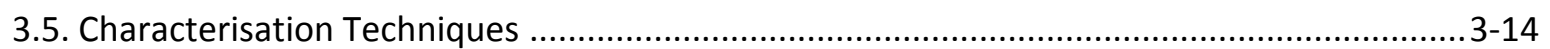

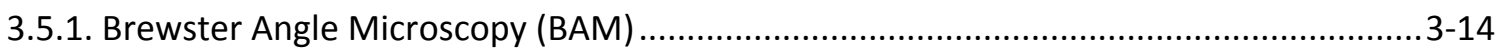

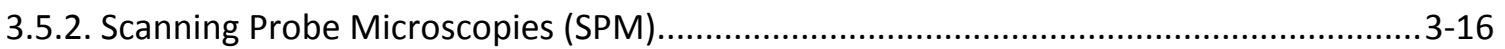

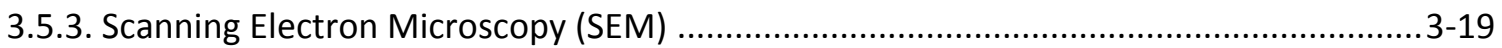

3.5.4. Photoluminescence, Raman and Fluorescence Spectroscopies ..................................... 3-21

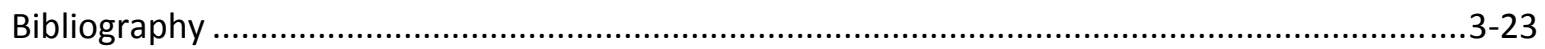

\section{Chapter 4: Unique Identification with Resonant Tunneling Diodes}

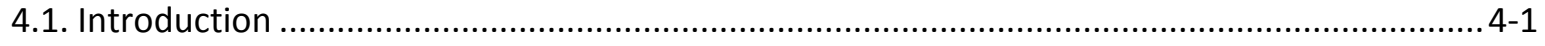

4.2. Uniqueness in Semiconductor Nanostructures .................................................................... 4-2

4.2.1. A Sensitivity to Quantum Confinement ........................................................................... 4-2

4.2.2. Imperfections and Defects in Nanostructures ................................................................. 4-3

4.2.3. Quantum Dots as an Example System ........................................................................... 4-4

4.3. Electronic Unique Identification using Quantum Confinement..............................................4-6

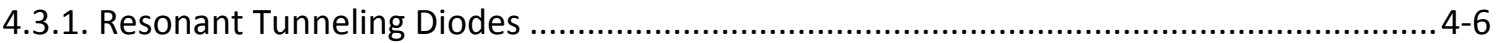

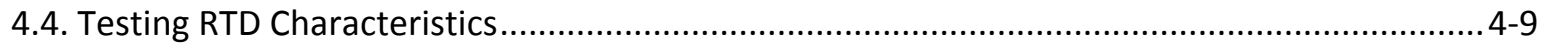

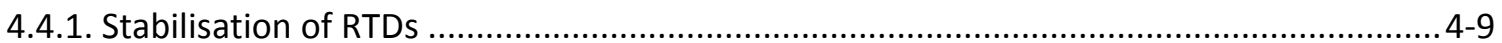

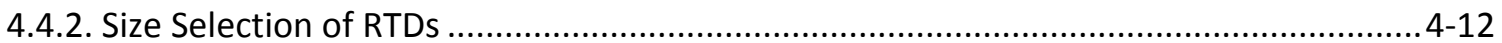

4.4. Resonant Tunneling Diodes as Unique Identifiers ...................................................................15

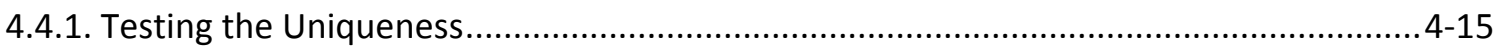

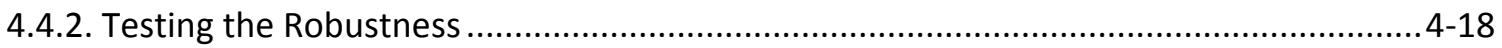

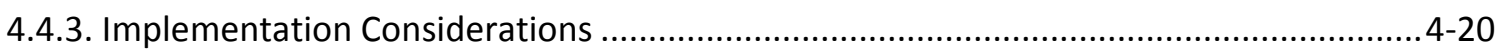

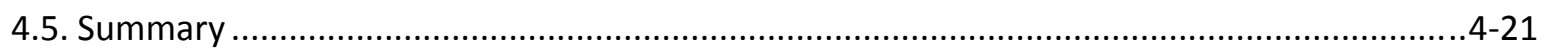




\section{Chapter 5: Langmuir-Blodgett Deposition of 2D Materials for Unique Identification}

5.1. Introduction 5-1

5.2. Unique Properties of 2D Materials . -2

5.2.1. An Introduction to 2D Materials 5-2

5.2.2. Effects of Confinement in 2D Materials . -2

5.3. Langmuir-Blodgett Films of 2D Materials ................................................................

5.3.1. A Brief Comparison of Fabrication Methods ......................................................... 5-6

5.3.2. Thin Films of Liquid Exfoliated 2D Materials......................................................... $5-7$

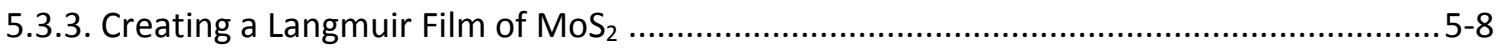

5.3.4. $\mathrm{MoS}_{2}$ at the Air-Water Interface ................................................................. 5-13

5.3.5. Deposition of a Langmuir-Blodgett Film .......................................................... 5-17

5.3.6. Creating Optical Identification Devices.............................................................. 5-18

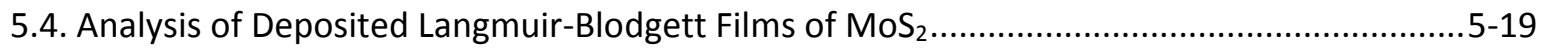

5.4.1. Quenched Photoluminescence of $\mathrm{MoS}_{2}$ Films ....................................................5-19

5.4.2. Investigation of Possible Quenching Mechanisms.....................................................5-21

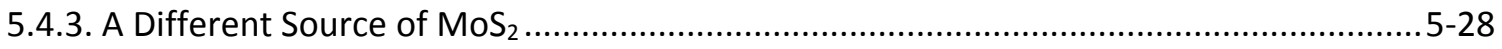

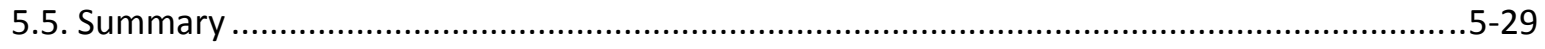

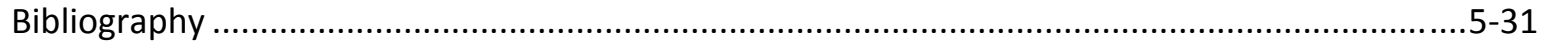

\section{Chapter 6: Building Optoelectronic Heterostructures with Langmuir-Blodgett Deposition}

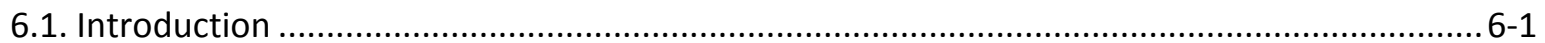

6.2. Materials for an Optoelectronic Device Architecture ...............................................................

6.2.1. An Introduction to Colloidal Quantum Dots ........................................................................

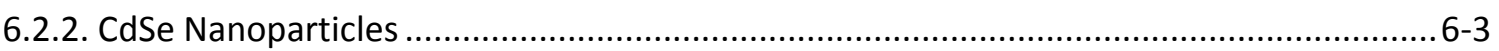

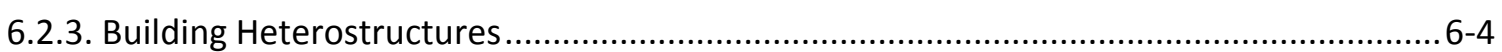

6.3. Langmuir-Blodgett Films of CdSe/Si Nanoparticles ..............................................................6-6

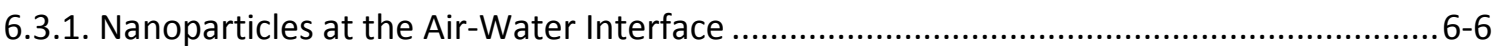

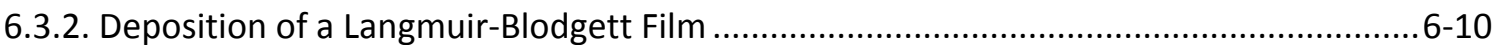

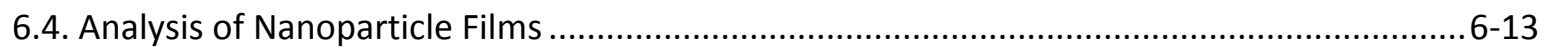

6.4.1. Investigation of Film Homogeneity ..........................................................................

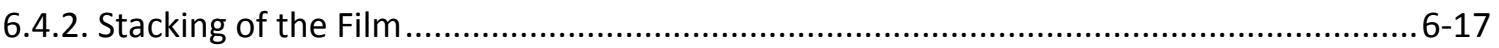

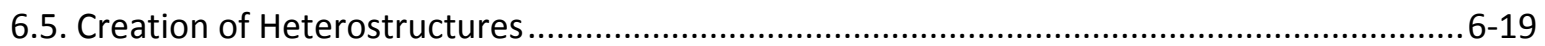

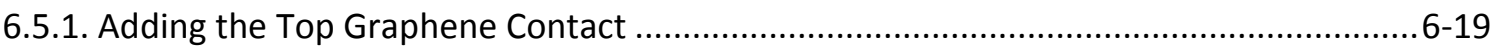


6.5.2. Analysis of Graphene/NP/Graphene Heterostructures

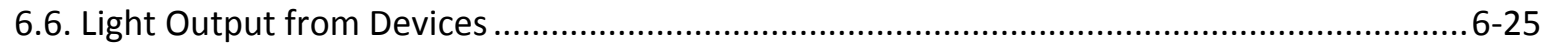

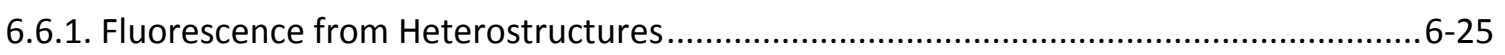

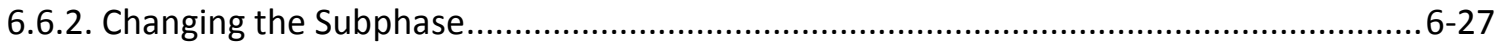

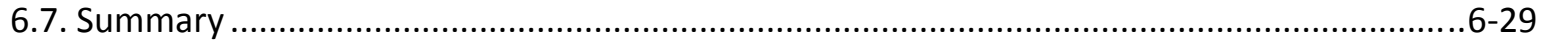

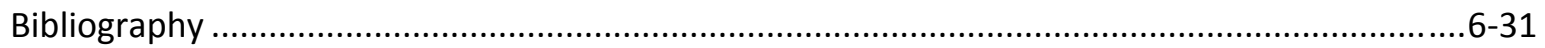

\section{Chapter 7: Conclusions and Future Work}

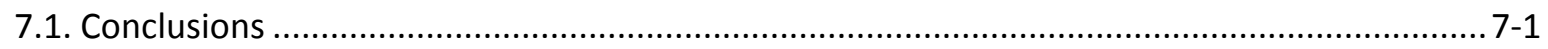

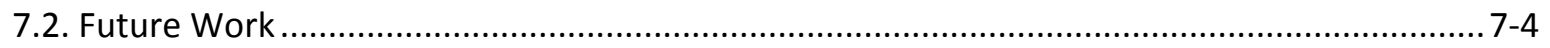




\section{Chapter 1}

\section{An Introduction to Security Based on Physical Disorder}

\subsection{Motivation}

The ever-growing number of connected smart devices, programs and data brings with it a growing demand to ensure the security and reliability of these systems. This problem is now a significant challenge for all of society, as these devices have become completely pervasive in everyday life. Example uses include carrying out financial transactions, communicating with other people, monitoring people's health and interacting with the environment. As these devices fulfil critical tasks, one of the core requirements that needs to be addressed lies in their secure authentication, identification and integrity checking. The rise of the Internet of Things (IOT), a connected web of networkable devices that come in all shapes and sizes has made this problem even more apparent. The authentication of such small, low-power devices has created a cause for concern considering how many of these devices will be connected over the next few years. Thus, it is of great societal, political and technological importance to create robust identification and authentication systems.

\subsection{The Need for a New Era of Authenticity}

The most vulnerable component in the security of an electronic system is normally the method of identity provision, which commonly relies on end users providing a password or some other unique data, such as a fingerprint. Unfortunately, it is well accepted that users are a weak link when it comes to security ${ }^{1}$ and alternative authentication schemes based on the storage of secret keys in electronic memory are employed. In this case, the critical point of vulnerability lies in their hardware 
implementation and key storage, and a host of attacks have been developed over recent years to extract, estimate and clone secret keys stored in memory. Once these keys are known, an attacker can then use this key to authenticate himself as a legitimate user or device ${ }^{2}$. A memory component can be shielded by adding some tamper-resistance, but this is both difficult and expensive. This inadequacy is especially poignant in embedded and low power mobile systems where an attacker could effectively gain full access to the device, as would be the case for a large majority of devices on the IoT. An alternative strategy has emerged over recent years, which utilises inherent, hard-to-clone randomness of physically disordered systems to define the secure identity of the system. This has been established as a promising alternative to the classical security approaches mentioned above, with two distinct types being proposed, known as unique objects (UNOs) and physically unclonable functions (PUFs).

\subsection{Using Hardware as a Basis of Trust}

\subsubsection{Employing Physical Disorder}

UNOs and PUFs comprise of a unique, hard-to-predict physical structure that is difficult to replicate, even by the manufacturer. As each contrived device is distinctive, they can then be used for unique authentication and identification. Furthermore, to tackle the key problem mentioned in the previous section, no information needs to be stored in digital memory and the identity can be extracted by probing the device when required. Such devices provide an intimate link between their structure and their identity, forming a robust footprint from which reliably secure systems may be built.

There are two important metrics that describe the usefulness of UNOs and PUFs, which are related to the uniqueness and robustness of the system. For example, a physically disordered system must be able to generate a unique 'fingerprint' not comparable to any other system, whilst providing a consistent measurement of its structure such that the same 'fingerprint' is measured each time. These metrics are frequently quantified by the average Hamming distance (HD), which quantifies: 1 . the similarities between the 'fingerprints' of two measurements of the same UNO/PUF (intra-device HD), 
and 2. the differences in the 'fingerprints' of two separate UNO/PUFS (inter-device HD), under the same measurement criteria ${ }^{3}$. In an ideal UNO/PUF, the system would have a large inter-device HD and a small intra-device HD, resulting in large differences between measurements of different devices (uniqueness), but only small distances when the same device is re-measured (robustness). The 'fingerprints' extracted from these systems can be used for unique identification and authentication providing they are unique and stable over varying environmental conditions. To this end, error correcting codes can be used to ensure this, or alternatively, the device's operational conditions can be averaged and/or calibrated ${ }^{4,5}$.

\subsubsection{Physically Unclonable Functions}

The first important class of physically disordered systems are physically unclonable functions (PUFs). A PUF is a physically unique system that can be probed with a range of different challenges (inputs), denoted $C_{n}$, which interact with the internal structure in a complex manner giving unique responses (outputs), denoted $R_{n}$. Examples of different challenges can include simple changes such as various angles of excitation in an optical system, or distinct voltages applied to an electronic system. To implement a PUF, upon its manufacture a range of unique challenges are sent to the device and the produced responses are measured. These challenge-response pairs (CRPs) must be unpredictable, unique and repeatable and it should be impossible to create another device with exactly the same CRPs. An online database is employed to store these CRPs, with a single CRP being used when authentication is required, as illustrated in fig 1.1. This authentication method requires an online database, and can also be applied to UNOs. An alternative authentication approach pioneered by Simmons uses a certificate of authenticity ${ }^{6}$. Here, the PUF (or UNO) is attached to the object along with a label containing some complementary information about the item. The inclusion of this complimentary information allows a protocol to be defined in which the PUF can be verified without the requirement of online access to a database, allowing for easier integration of the PUF (or UNO) into any architecture. The term PUF was first formerly used in ref [7], but were originally given the denomination physical one-way functions ${ }^{8}$. 
Chapter 1 - An Introduction to Security Based on Physical Disorder The security requirements of a PUF require that it be intractable for an attacker to be able to create a physical or a software clone of the PUF. For different security demands, PUFs can be further separated into two separate categories, namely weak PUFs (also known as physically obfuscated keys (POKs)) and strong PUFs. In a nutshell, the distinction is in the number of CRPs, which varies polynomially or exponentially with the number of elements (such as the number of flip-flop cells in a Static RAM chip) in the system, respectively. Weak PUFs must allow:

- Challenge-Response Pairs: A weak PUF probed with one (very few) fixed challenge(s), must give a random response(s) dependent on the internal disorder.

- Key Derivation: The responses extracted from a weak PUF can then be used as a digital key for standard security applications.

- Practicality and Operability: The weak PUFs responses must be stable under different environmental conditions and multiple readings.

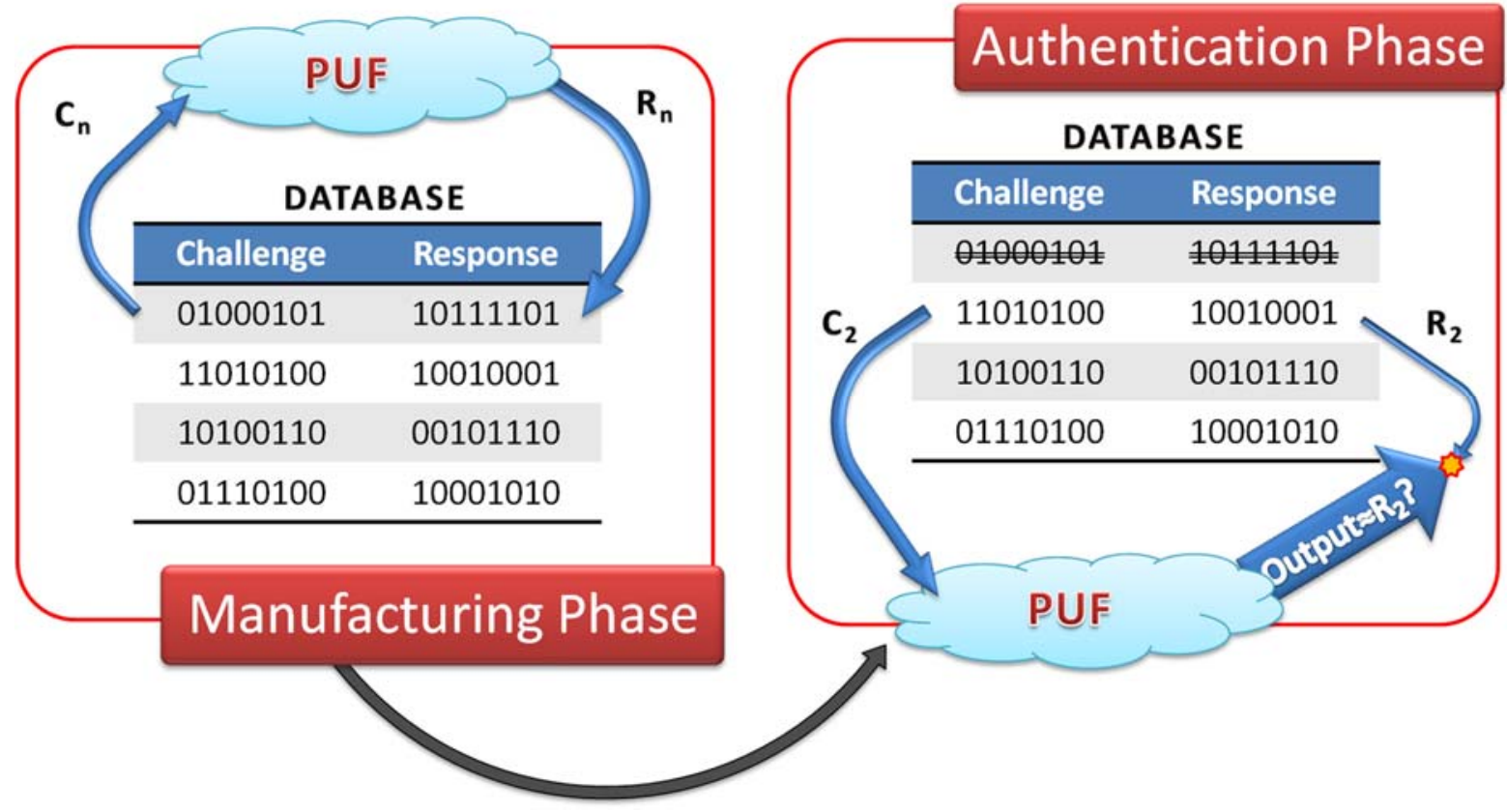

Figure 1.1: Implementation of a PUFs challenge-response pairs (CRPs). During the manufacturing phase (left), the CRPs of a PUF are measured by the manufacturer and stored in a database. When authentication is required (right), the device picks a challenge at random from the database and sends it to the PUF in question, if the produced response from the PUF agrees with the response stored in the database, then a legitimate device is present. 


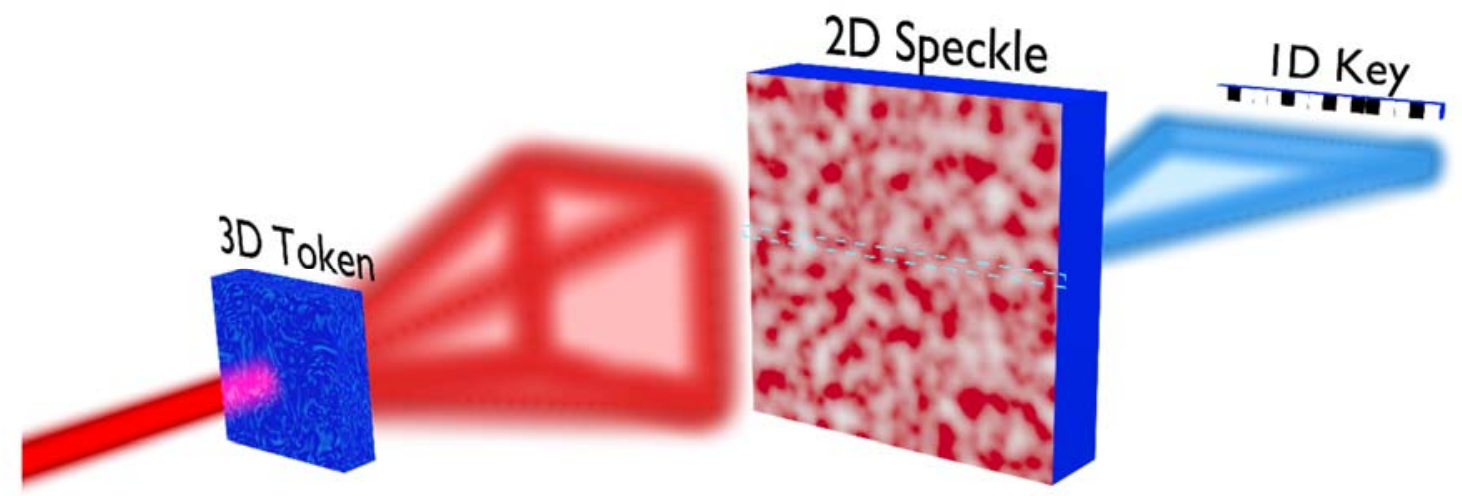

Figure 1.2: Example of an optical scattering token that depending on implementation can be used as either a weak or strong PUF. Here, a light source (normally a laser) illuminates the token at a specific angle and the resulting speckle pattern depends on the internal order of the token. For the generation of the response, the random speckle pattern produced by the PUF is input into a hash function to create a key that can be used for cryptographic purposes.

Strong PUFs have similar requirements for the CRPs, though the number must be extremely large and commonly varies exponentially with some aspect of the system, such as the number of components used in constructing the PUF. Strong PUFs must contain two additional properties:

- Access Mode: There is no restriction on access to the PUF, and an attacker with access can apply multiple challenges and measure multiple responses at will.

- Security: There must be a low probability that an attacker (or the manufacturer) can predict the response to a random challenge. This must hold even if an attacker is given the PUF for a long period of time prior to implementation, and is able to make any reasonable physical measurement including determination of a large subset of CRPs.

Whilst strong PUFs allow the attacker to gain full access and measure the properties of these systems, the opposite holds for weak PUFs. Here, the responses are measured internally by embedded hardware such that the derived secret is never revealed. This is a requirement dictated by the existence of only a few CRPs in these systems, which if revealed would render the security void.

The applications of PUFs include cryptographic solutions, authentication and identification. For 
example, PUFs can be used to generate low cost keys for identification and cryptographic algorithms, or the binding of hardware to software platforms. A variety of devices have been implemented for use as PUFs, notwithstanding; scattering in an optical medium (illustrated in fig 1.2$)^{8}$, modes in silicon ring oscillators ${ }^{7}$, statistical delay variations between nominally identical paths ${ }^{9}$ and static random access memory cells ${ }^{10}$. However, it has been shown that certain types of PUF have been vulnerable to cloning, simulation and other attacks. For example, Helfmeier et al. successfully cloned an SRAM PUF in a time of 20 hours $^{11}$, arbiter PUFs and their derivatives are susceptible to machine learning ${ }^{12}$ and a range of other PUFs are vulnerable to side-channel attacks $^{13}$

\subsubsection{Unique Objects}

A unique object (UNO) is a device with a small set of inimitable properties that can be measured by an external apparatus, to produce a single 'fingerprint'. It is, in effect, a weak PUF with one CRP. The measurement apparatus that is required to measure the UNO needs to do so quickly and efficiently; and preferably be inexpensive to fabricate. The security of a unique objects relies on the impossibility of re-fabrication. It is assumed that an attacker can know everything about the UNO including the 'fingerprint' and the internal structure itself but unless a clone can be fabricated, the UNOs security remains intact. The specific requirements of a UNO are:

- Disorder: The unique identity must be based on the disorder of some physical object.

- Operability: The fingerprint must be robust against aging, changing environmental conditions and repeated measurements. Whilst the external measurement apparatus must be able to be recreated to allow the same characterisation possibility of any UNO in a practically and economically viable method.

- Unclonability: A unique physical object that displays the same fingerprint must be prohibitively expensive or impractical to produce, even by the manufacturer.

Many different types of system have been proposed for use as UNOs, with some examples being sprayed random surfaces, CDs/DVDs, optical signatures and DNA ${ }^{14,15,16,17}$. No secret information needs 
to be stored when implementing a UNO, which largely reduces cost requirements compared to standard key storage. However, a critical measurement is how much the 'fingerprint' changes when some of the internal structure is marginally altered. This factor dictates how exact an attacker would need to be when replicating such a device, and can be used as a good comparator between different UNO implementations. The concept of UNOS is well established and has been used for more than three decades with obvious examples including confidential documents such as passports, ID cards and bank notes.

\subsubsection{Physical Disorder at the Extreme}

To circumnavigate the problems of cloning in conventional UNO and PUF structures, it has been proposed in this work to make use of nanostructures exhibiting quantum confinement for the first time. By employing low-dimensional nanostructures, the readout of a complex identity with the minimum amount of resources can be achieved, as the atomic make-up of the device dictates the physical properties of the system. Previous works have concentrated on the quantum readout of PUFs, which requires the preparation and measurement of a quantum state that is passed through a physically disordered structure ${ }^{18}$. In particular, this uses a complex setup that incorporates spatial light modulators that transform the incoming quantum state of light. The uniqueness of this system is attributed to the no-cloning theorem, making it impossible to copy the incoming challenge. Incorporating quantum light into a practical system is not only challenging, but requires precise alignment of the expensive optical components, whilst being spatially hard to implement due to size restraints. Here, the converse is proposed, in which an easy-to-measure macroscopic property is sent into a system that exhibits quantum confinement and subsequently re-measured; this offers greater practicality and implementation potential. The unique measurable property is extremely sensitive to the confinement effects of the system, which is highly dependent on the atomic structure, composition and interfaces of the system. These devices would extract their unique identity from this unique arrangement of atoms, and as such would be the hardest possible system to clone to date. 


\section{Bibliography}

1 "Security Engineering: A Guide to Building Dependable Distributed Systems", R. Anderson, John Wiley \& Sons, New Jersey (2008).

${ }^{2}$ A. Barenghi et al., "Fault Injection Attacks on Cryptographic Devices: Theory, Practise and Countermeasures", Proc. IEEE, 100, 3056 (2012).

${ }^{3}$ R. W. Hamming, "Error Detecting and Error Correcting Codes", Bell Sys. Tech. Journal, 29, 2 (1950).

${ }^{4}$ M. D. Yu \& S. Devadas, "Secure and Robust Error Correction for Physical UnclonableFunctions", IEEE Design Test Comput., 27, 48 (2010).

${ }^{5}$ M. Majzoobi et al., "FPGA PUF Using Programmable Delay Lines", IEEE Workshop on Information Forensics and Security, 1-6 (2010).

${ }^{6}$ G. J. Simmons, "A System for Verifying User Identity and Authorization at the Point-of sale or Access", Cryptologia, 8, 1 (1984).

7 B. Gassend et al., "Silicon Physical Random Functions", Computer and Communication Security Conference (2002).

${ }^{8}$ R. Pappu et al., "Physical One-way Functions", Science, 297, 2026 (2002).

${ }^{9}$ D. Lim et al., "Extracting Secret Keys from Integrated Circuits", IEEE Transactions on VLSI Systems, 13, 1200 (2005).

${ }^{10}$ D. E. Holcomb et al., "Power-up SRAM State as an Identifying Fingerprint and Source of True Random Numbers", IEEE Transactions on Computers, 58, 1198 (2009).

${ }^{11}$ C. Helfmeier et al., "Cloning Physically Unclonable Functions", IEEE International Symposium on Hardware-Oriented Security and Trust (2013).

12 U. Rürrmair et al., "Modelling Attacks on Physical Unclonable Functions", Conference on Computer and Communications Security (2010).

${ }^{13}$ D. Merli et al., "Side Channel Analysis of PUFs and Fuzzy Extractors", Conference on Trust and Trustworthy Computing (2011).

${ }^{14}$ S. Graybeal \& P. McFate, "Getting out of the STARTing Block" Scient. Am., 261, 6 (1989).

${ }^{15}$ G. Hammouri et al., "CDs Have Fingerprints Too", Cryptographic Hardware and Embedded Systems, 348-362 (2009).

${ }^{16}$ U. Ruhrmair et al., "Method and System for Security Purposes", European Patent Application, EP09157041.6 (2009).

${ }^{17}$ C. Clelland et al., "Hiding Messages in DNA Microdots" Nature, 399, 6736 (1999).

${ }^{18}$ B. Skoric, "Quantum Readout of Physical Unclonable Functions", Progress in Cryptology (2010). 


\section{Chapter 2}

\section{An Introduction to Semiconductors and Quantum Confinement}

\subsection{Introduction}

In this thesis, atomically imperfect structures will be studied to prove that such systems can provide adequate variation for unique identification, as the sensitive effects of quantum confinement exhibited in these low-dimensional structures is directly related to the order on this scale. Utilising the measurement of a quantum effect amplifies the influence of these atomic-scale defects, and provides a simple way to measure them simply and reliably. This work will lay the foundations for UNOs and PUFs comprising of such structures to be developed.

To realise this objective a number of different systems have been studied, with semiconducting materials at the core of each. The electronic and optical properties of such systems are determined by their band structure, composition and the interaction of excited carriers with their environment. Thus, this chapter describes the main properties of semiconductors and the resulting effects that quantum confinement has on the system. The chapter is concluded by providing an overarching description of the work's main goals followed by an outline of the thesis.

\subsection{Semiconducting Materials}

\subsubsection{The Basics}

A semiconductor is a solid material with a periodic crystalline structure that, at absolute zero, is electrically insulating, whilst being conductive for temperatures below its melting point. In a semiconductor, the wavefunction of the electrons interacts with the periodic potential of the crystal, 
resulting in a 'smearing' of the energy levels into bands (such as those in fig 2.1). This interaction also results in the creation of regions with prohibited energies in which electrons cannot exist, this is known as the bandgap of the material.

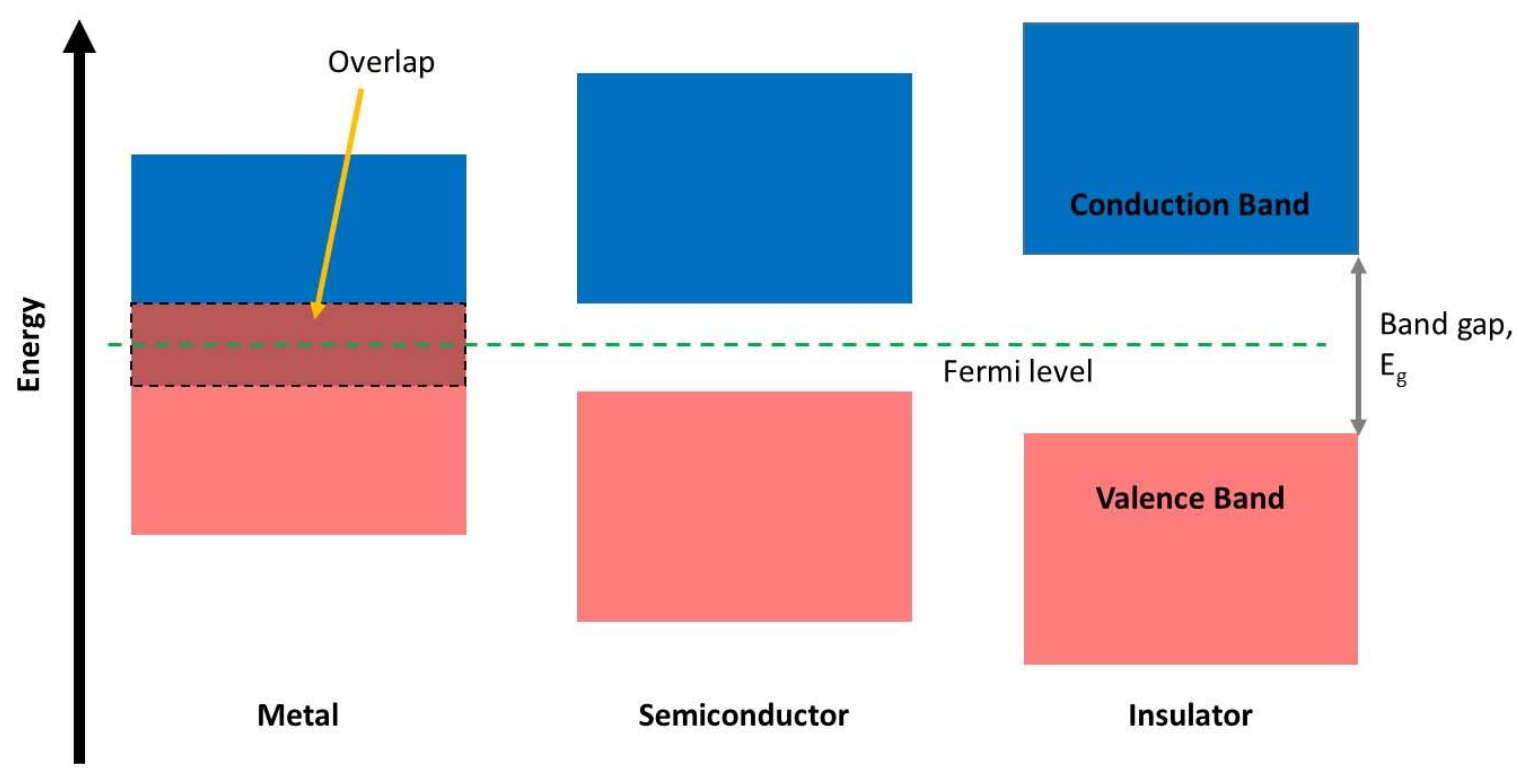

Figure 2.1: Variation of the conduction and valence bands of a metal, semiconductor and insulator. Displaying the overlapping bands in metals, and small and large band gaps in semiconductors and insulators, respectively.

The most important energy bands in a crystal structure are the bands closest to the Fermi level, known as the valence band (VB) and conduction band (CB). These correspond to the bands with the highest occupied electronic states and lowest vacant electronic states at absolute zero, respectively. The Fermi level is the equilibrium position, defined as the energy when the probability of an energy level being occupied by an electron at a given temperature is $50 \%$. Deviations from equilibrium have the largest effect on the $\mathrm{VB}$ and $\mathrm{CB}$, meaning they alone can be used to describe the majority of the interesting properties of a material. The typical energy bands of metals, semiconductors and insulators are shown in fig 2.1. The overlap seen in the VB and CB of metals give them their highly conducting properties, as the electrons can easily flow into states in the conduction band. The energy gap between the bottom of the CB and the top of the VB denotes the band-gap, Eg. For insulators, this gap is large which prevents any current from flowing through the material. However, in semiconductors, 
this gap is relatively small and the conductivity depends on the $C B$ and $V B$ of the specific semiconductor material in question ${ }^{1,2}$. Current can flow in a semiconductor as the electrons may be excited from the $V B$ to the $C B$ by thermal energy or electromagnetic radiation, creating empty states for charge carriers to flow into. When an electron is excited from the VB to the $C B$, a vacancy is left behind in the VB; known as a hole (see fig 2.3a). A hole is a quasi-particle that has an effective mass determined by the band structure of the semiconductor and has a charge opposite to that of the electron. The electron and the hole can both contribute to charge conduction in semiconductors. Moreover, due to their opposite charges, these particles can also be attracted to one another to create an electron-hole quasiparticle, known as an exciton, which will be discussed later in the chapter.

\subsubsection{Doping in Semiconductors}

The probability of thermally exciting an electron from the VB to the CB is governed by Fermi-Dirac statistics with respect to the Fermi level. Here, the probability, $P(E)$, of an energy level, E, being occupied is:

$$
P(E)=\frac{1}{1+\exp \left(\frac{E-E_{F}}{k T}\right)}
$$

where $\mathrm{E}_{\mathrm{F}}$ is the Fermi level, $\mathrm{k}$ is the Boltzmann constant and $\mathrm{T}$ is the temperature ${ }^{3}$.

For an undoped semiconductor in thermal equilibrium, and at absolute zero, the Fermi level will always be found halfway between the VB and the $C B^{4}$. This is also the case for insulators, but due to the larger bandgap, the probability of exciting electrons is negligible. The Fermi-Dirac distribution can be seen in fig 2.2. At absolute zero, the probability that an electron can lie in a state above the Fermi level is zero, whereas below the Fermi level it is equal to one. This gives us another definition for the Fermi level; the maximum energy that an electron may have at absolute zero. As the temperature is increased, the electrons may be excited into the conduction band and the probability of occupation above the Fermi level thus increases. To conserve the total electron population, the probability of occupation of states below the Fermi level must decrease at the same rate. 


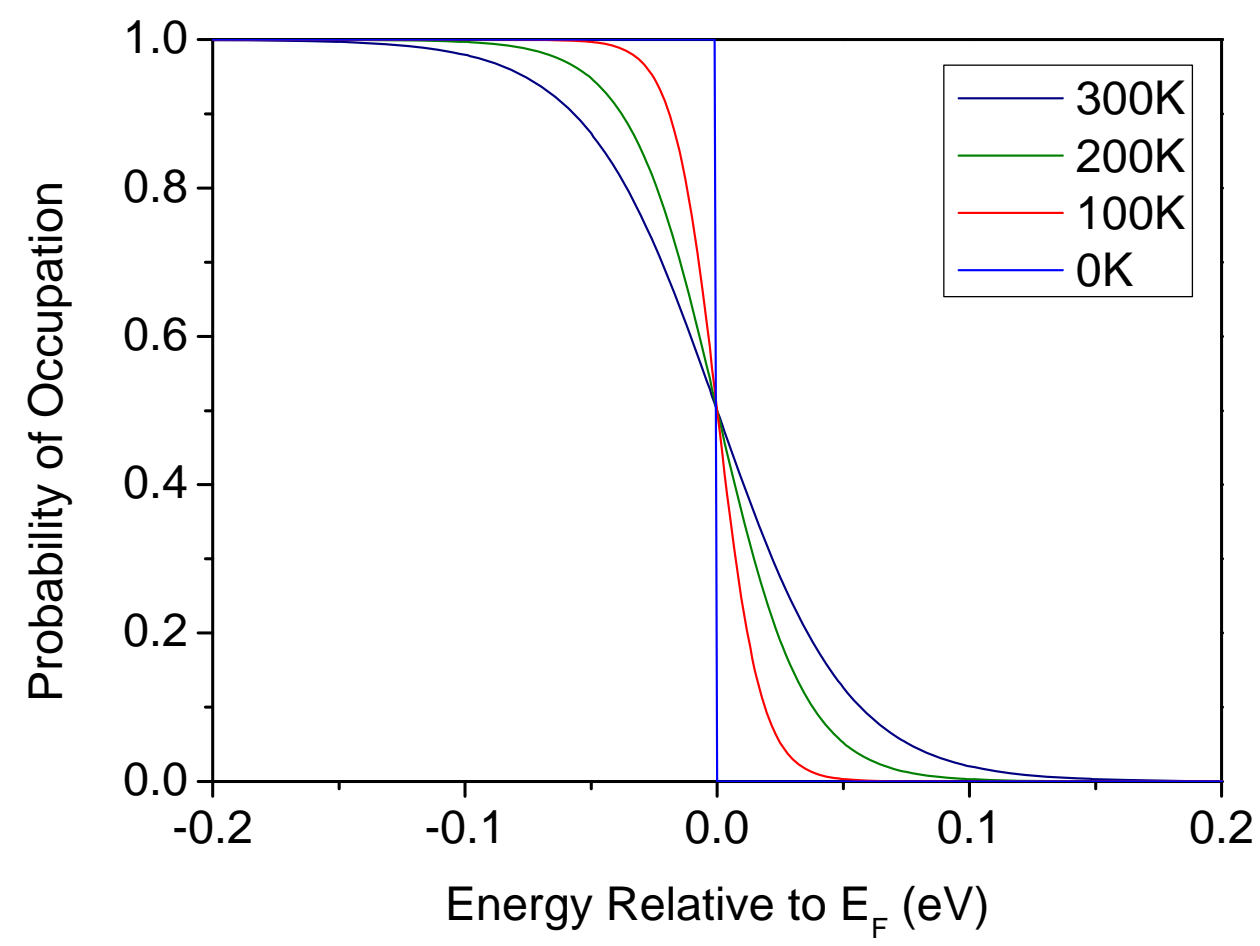

Figure 2.2: Fermi-Dirac statistics showing the distribution of electrons in semiconductors, relative to an arbitrary Fermi level for temperatures of $0 \mathrm{~K}, 100 \mathrm{~K}, 200 \mathrm{~K}$ and $300 \mathrm{~K}$.

To increase the conductivity of a semiconductor, the material can be doped with elements that contain more or less valence electrons than the native material it is replacing. Dopant atoms that contain fewer electrons than the host material are known as acceptors. Ideal acceptors have an unoccupied energy level that sits just above the VB of the semiconductor crystal, as illustrated in fig 2.3b. The energy difference between the VB and the acceptor level ( $A L)$ is important as the size of the gap controls the probability that electrons can be thermally excited into this level. The holes that are subsequently left in the VB are now free to move, which makes the material conductive (for holes). This mechanism is known as p-type doping and results in the Fermi level shifting towards the VB. On the other hand, dopant atoms which contain a greater number of electrons than the host material are known as donors. Ideal donors contain an occupied electron level that lies just below the conduction band as in fig 2.3c. Similarly, the location of the donor level (DL) is extremely important as it controls the rate in which charge carriers are excited into the conduction band ${ }^{5}$. 

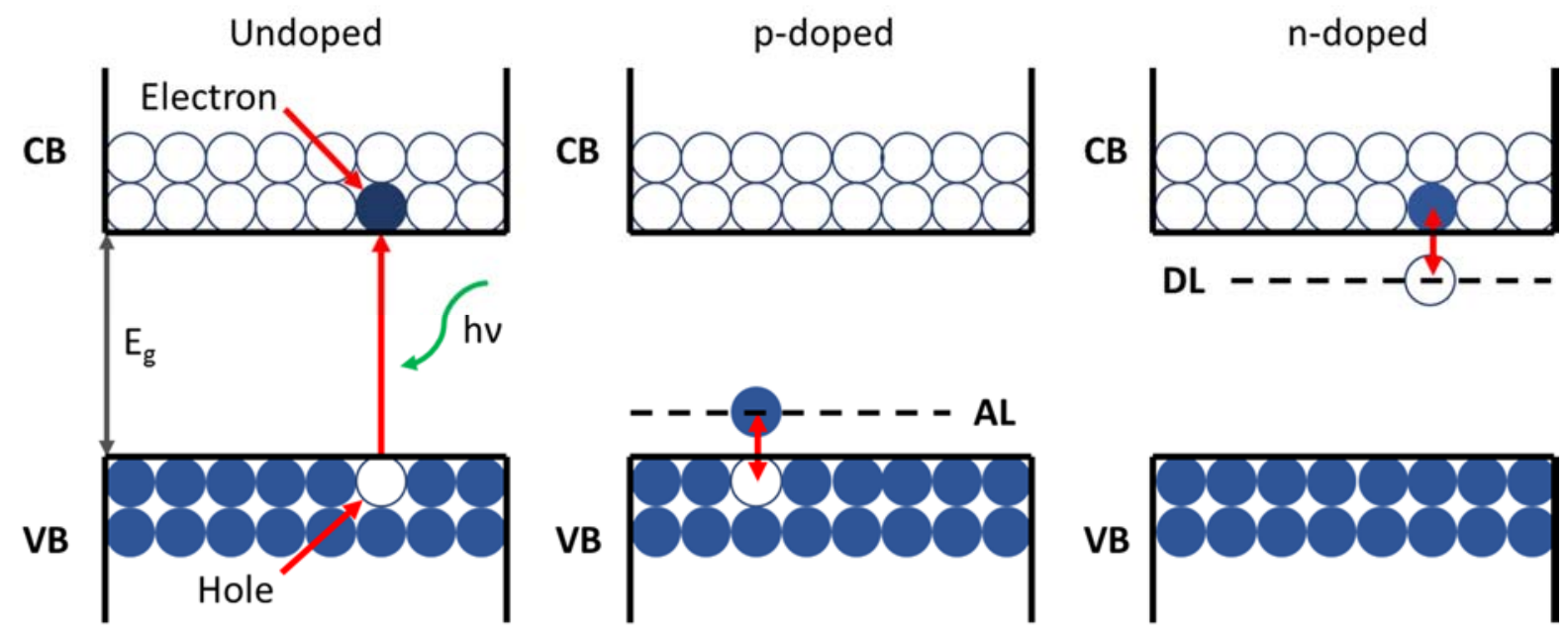

Figure 2.3: Doping effects in the occupied levels of semiconductors, with (a) excitation of a single electron in an undoped crystal with a quanta of energy, hv, (b) a p-doped crystal, and (c) a n-doped crystal. Electrons and holes are indicated and the conduction band (CB), valence band (VB), acceptor level (AL) and donor level (DL) are labelled.

If the energy gap is sufficiently small (less than the thermal energy at room temperature; $\sim 25 \mathrm{meV}$ ) then electrons may be excited into the $\mathrm{CB}$ and form a material that is conductive (for electrons) at room temperature. This is known as n-type doping and results in the Fermi level shifting towards the CB.

\subsubsection{Semiconductor Band Structure}

Due to the periodic and highly symmetric crystalline structure of semiconductors, a unit cell can be defined which can be used to describe the entire crystal lattice, by repeating the unit cell throughout the bulk of the material. This also allows the definition of a reciprocal lattice that is able to provide information about the relative angles between planes and the thickness of these planes in the real semiconductor crystal. Where the unit cell is used to define the bulk crystal, Brillouin zones are used to define the reciprocal lattice ${ }^{6,7}$. As an example, the first Brillouin zone for a face centred cubic crystal is shown in fig 2.4 , with the points of symmetry highlighted. The points represented in reciprocal lattice space correspond to a wavevector in the real lattice space. At $\Gamma$, the zone centre, the wavevector and therefore the momentum, is zero, and as the distance away from the zone centre increases, there is a linear increase in momentum. 


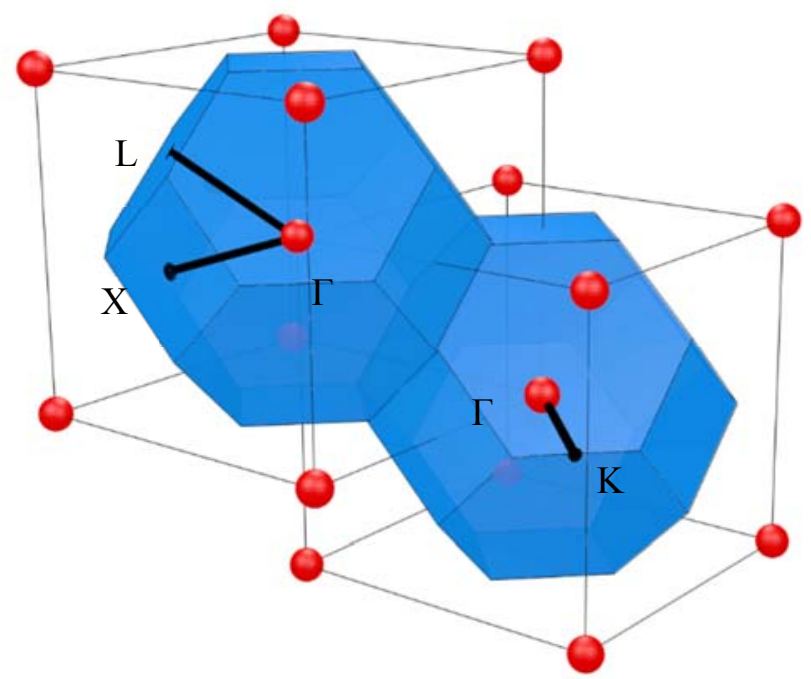

Figure 2.4: The first Brillouin zone for a face centred cubic crystal lattice, with the points of symmetry labelled as $\Gamma$ (the zone centre), $\mathrm{X}, \mathrm{K}$ and $\mathrm{L}$.

As the symmetry of the crystal changes depending on which path is followed through the lattice, a moving electron has an energy that is dependent on its direction of motion. The symmetry points highlighted in fig 2.4 act as a convenient reference when discussing the energy of an electron as a function of the wavevector, or momentum. Plotting an electron's energy in this way is known as a dispersion relation. Such dispersion relationships for the two most common semiconductors used for electronic or optical devices, silicon ( $\mathrm{Si}$ ) and gallium arsenide (GaAs), are shown in fig 2.5. The bands that correspond to the valence band; heavy-hole $(\mathrm{HH})$, light-hole $(\mathrm{LH})$ and split-off $(\mathrm{SO})$ bands are shown, in addition to the conduction band and the band gap. The origins of these bands will be discussed below. The complex form of these dispersion relationships arises due to the charge carriers' interaction with the crystal lattice ${ }^{8}$. However, at the points corresponding to the VB maximum and CB minimum where the momentum is approximately zero, the dispersion relationship can be estimated as parabolic. To this end, the dispersion relationship for an electron in free space is used:

$$
E=\frac{\hbar^{2} k^{2}}{2 m}
$$

where $k$ is the wavevector, $m$ is the free-space mass of an electron and $\hbar$ is the reduced Planck constant. However, the interaction of the electron in a crystal lattice leads to changes of its mass, and 
the electron behaves as though it has a reduced mass. This mass is known as the effective mass, and can be calculated (for electrons and holes) using the above equation and the band structure of the material system. The definition of the effective mass is thus:

$$
m^{*}=\frac{\hbar^{2}}{d^{2} E / d k^{2}}
$$

where the denominator defines the local curvature of the dispersion relationship for a given material. For the band structures in fig 2.5 , the effective mass of the electron has been calculated as $0.2 m_{0}$ and $0.067 \mathrm{~m}_{0}$ for $\mathrm{Si}$ and GaAs at the minima of their conduction bands, respectively, where $\mathrm{m}_{0}$ is the freeelectron mass ${ }^{2,9}$. This also allows the definition of the $\mathrm{HH}$ and $\mathrm{LH}$. As it can be seen in fig 2.5 , the local curvature for these bands is slightly different, resulting in a 'heavy' effective mass and a 'light' effective mass. For Si (GaAs), the heavy and light hole masses are $0.49 \mathrm{~m}_{0}\left(0.45 \mathrm{~m}_{0}\right)$ and $0.16 \mathrm{~m}_{0}\left(0.082 \mathrm{~m}_{0}\right)$, respectively ${ }^{2,10}$.
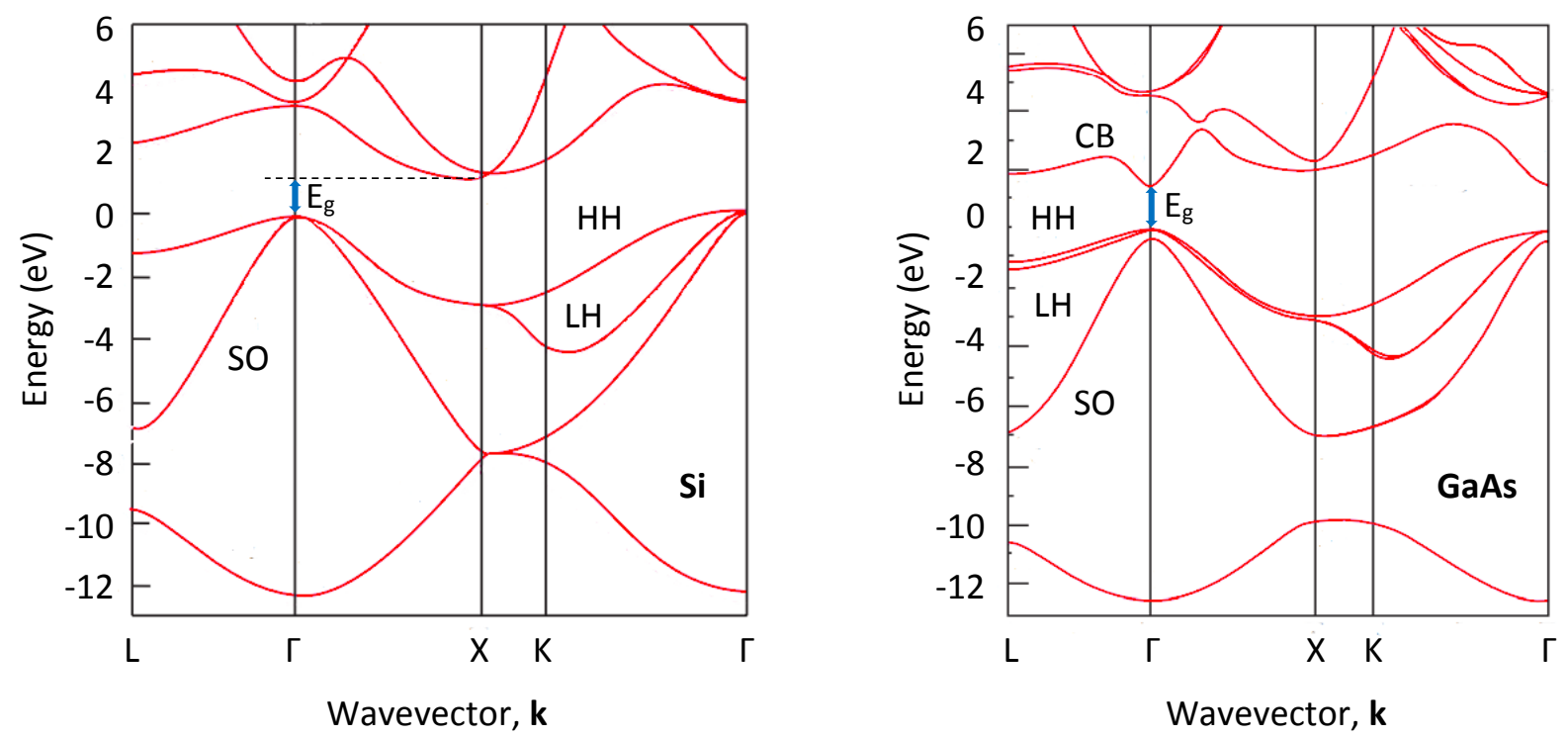

Figure 2.5: Electronic band structure of (a) silicon and (b) gallium arsenide. Showing the heavyhole, light-hole and split-off bands of the valence band, the conduction band and the band gap. [Reprinted with permission from Springer: from [7] (copyright 2016).]

The $\mathrm{HH}$ and $\mathrm{LH}$ bands overlap at the zone centre, as they share the same total orbital angular momentum quantum number $(J=3 / 2)$. The splitting into separate bands is due to the angular momentum projections, where $m_{j}= \pm 3 / 2$ for the $H H$ and $m_{j}= \pm 1 / 2$ for the $L H$. On the other hand, the 
split-off band ( $\mathrm{SO}$ ) has a total angular momentum quantum number of $\mathrm{J}=1 / 2$, leading to a reduction in energy. Commonly, the separation of this band from the others results in the properties of this band being negligible to the resulting properties of the semiconductor. This is very different to the case of the $\mathrm{CB}$, as the electrons have no orbital angular momentum and so the properties of this band are solely determined by the electron spin. Thus the angular momentum projections of this band are $m_{j}= \pm 1 / 2$.

\subsubsection{Direct vs. Indirect Semiconductors}

Through observation of fig 2.5, it can be seen that the minimum of the $C B$ and the maximum of the VB are not always at the same wavevector. In the dispersion relationship for GaAs, these points both coincide at the zone centre resulting in what is known as a direct-gap semiconductor. Alternatively, in the case of $\mathrm{Si}$, the positions of the minimum of the $\mathrm{CB}$ and maximum of the $\mathrm{VB}$ lie at the $\mathrm{X}$ and $\Gamma$ points, respectively. In this case, the system is known as an indirect-gap semiconductor. This attribute has implications on the optical properties of the material. For example, if a photon with energy larger than $E_{g}$ was to impinge on GaAs, an electron could be excited from the $V B$ to the $C B$ as the electron would not need to gain any momentum. However, if the same principle was applied to silicon, there would need to be a change in momentum for the electron to be excited across the band gap. Importantly, this cannot come from the photon itself which has negligible momentum. The additional momentum required is provided through phonons; a vibrational quanta of the crystal lattice. Thus, for a transition to occur in an indirect semiconductor, a photon and a phonon must be absorbed or emitted. As the number of phonons present in the system depends on temperature, this leads to a large temperature dependence of the absorption coefficient of indirect semiconductors. The absorption coefficients of silicon and GaAs at room temperature are $1 \times 10^{1} \mathrm{~cm}^{-1}$ and $8 \times 10^{3} \mathrm{~cm}^{-1}$ at the wavelengths corresponding to their band gap energies, respectively ${ }^{11,12}$. Silicon's absorption can be enhanced by using high energy photons to excite electrons into states in the $C B$ that do not require the use of additional momentum.

The direct or indirect nature of the semiconductor material can also have implications on the light 
emission. In direct-gap semiconductors, an electron that has been excited into the CB can recombine radiatively by falling back into the $\mathrm{VB}$, whilst emitting a photon to conserve energy. For indirect-gap systems however, the recombination would be a second order process, requiring the emission of a photon and the absorption or emission of a phonon. This process has a much lower probability and gives indirect-gap semiconductors long radiative lifetimes. Furthermore, as the electron remains in its excited state for a larger period of time, there is also the chance of non-radiative recombination, through the assistance of defects, an Auger process or surface recombination ${ }^{13,14}$. This results in indirect semiconductors being bad light emitters and is the reason silicon is not used for such applications.

\subsubsection{Compound Semiconductors}

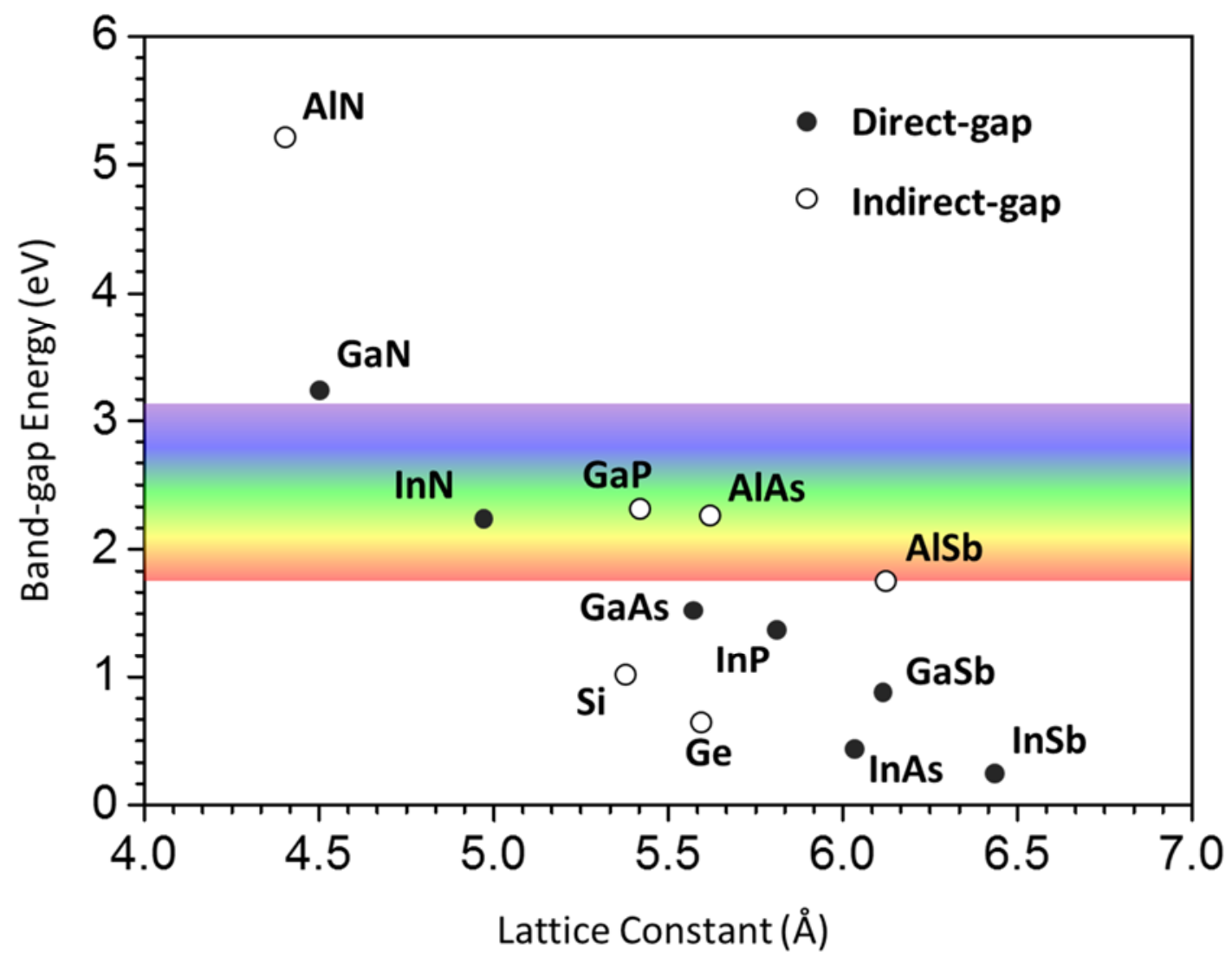

Figure 2.6: Band-gap vs. lattice constant relationship for the most common semiconductor materials.

In group IV semiconductors such as silicon, the band gap energy and the absorption rate is fixed for a given temperature; although such properties can be altered by alloying group IV materials, such as in 
SiGe. This flexibility is enhanced for compound semiconductors, such as the III-V's or II-VI's, which provide a range of bandgap tuning options. Most prominent is the capability of producing ternary and quaternary compounds, which change the band gap of the material $\left.\right|^{15}$. The effects of this can be seen in fig 2.6, which shows the band gaps of the most popularly used semicondcutor materials against their correspnding lattice constants. Producing ternary structures, for example with GaAs and AlAs, allows the band gap to be tuned along an approximate line between the two points. However, there are a number of requirements when building such materials. Mainly, the lattice constant must be approximately equal, as if large pertubations exist then this will create many defects within the semiconductor ${ }^{16}$. Also, if combining a direct gap and an indirect gap semiconductor, the percentage of the indirect material cannot be too great otherwise the resulting ternary alloy also becomes indirect ${ }^{17}$. Advantageously, when alloying materials in this way, the material contains the same number of valence electrons as group IV semiconductors, whilst remaining electrically neutral.

\subsection{The Effects of Confinement}

\subsubsection{The 'Particle in a Box' Approximation}

The electronic and optical properties of a system are effected by quantum confinement when the physical size of the system becomes comparable to the de Broglie wavelength of the electrons and holes ${ }^{18}$. If an electron is in motion through a semiconductor crystal with a temperature $\mathrm{T}$, the de Broglie wavelength is given by:

$$
\lambda_{d e B}=\frac{h}{\sqrt{m^{*} k_{B} T}}
$$

where $m^{*}$ is the effective mass of the electron or hole, $h$ is Planck's constant and $k_{B}$ is the Boltzmann constant. Thus, for an electron in a GaAs crystal (where $\left.m^{*}=0.067 m_{0}\right)$ at room temperature $(300 \mathrm{~K})$, the de Broglie wavelength is $\sim 42 \mathrm{~nm}$. This means that for nanostructures on the orders of tens of $\mathrm{nm}$, quantum confinement effects will govern the properties of the material.

In order to achieve this, epitaxial techniques are employed to produce layered heterostructures that 
contain semiconductor materials of differing bandgaps. For example, when a thin layer of material (tens of $\mathrm{nm}$ ) is sandwiched between two layers of another material with a larger band gap, a layer which confines the electrons and holes is formed; this is known as a quantum well (QW) and is represented in fig 2.7. Quantum confinement of the electrons and holes leads to energy states that are no longer continuous, but are semi-discrete; highlighted in fig 2.7. The discretisation of these energy levels can be predicted by the 'particle in a box' model. In its simplest form, the time independent Schrödinger equation is:

$$
-\frac{\hbar^{2}}{2 m^{*}} \frac{d^{2} \emptyset_{n}(z)}{d z^{2}}+V(z) \emptyset_{n}(z)=E_{n} \emptyset_{n}(z)
$$

where $V(z)$ is the structural potential seen by the particle along $z, m^{*}$ is the effective mass and $\phi_{n}$ and $E_{n}$ are the eigenfunction and eigenenergy for each value of $n$, the principal quantum number.

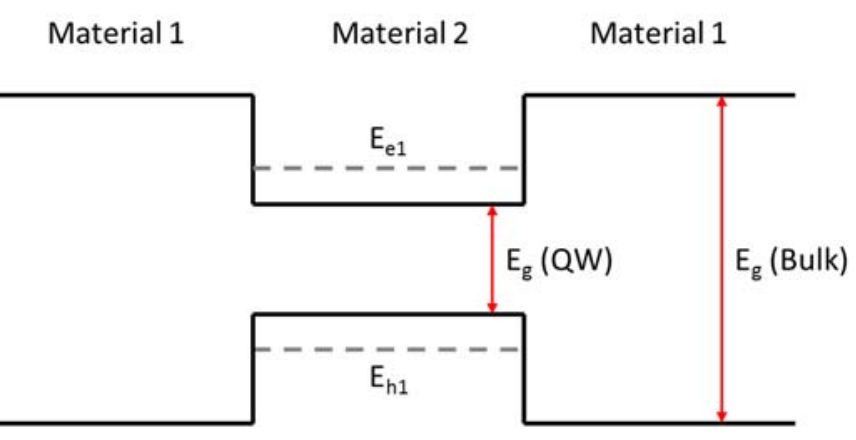

Figure 2.7: Different band-gap materials causing quantum confinement to form a quantum well. The energy levels of the first hole and electron, $E_{h 1}$ and $E_{\mathrm{e} 1}$, respectively are shown.

By considering an infinite barrier height (i.e. $V(z)=0$ in the well, $V(z)=\infty$ outside the well), where the wavefunction cannot exist outside the well, the solutions become particularly simple:

$$
E_{n}=\frac{\hbar^{2}}{2 m^{*}}\left(\frac{n \pi}{L}\right)^{2} \quad \emptyset_{n}=A \sin \left(\frac{n \pi z}{L}\right)
$$

where $L$ is the width of the quantum well. The first three energy levels that arise from these equations are shown in fig 2.8. It is important to notice that the energy levels of the well have an inverse square law with L. This attribute is the critical reason that devices exhibiting quantum confinement can be used for unique identification, as if the width of the well has atom-scale fluctuations along the 
interface by only 1 monolayer or less, this results in a shift of the energy level in the well, which can be measured macroscopically. For example, in a GaAs $5 \mathrm{~nm}$ quantum well, the addition of 1 monolayer results in an electron energy level red-shift of $43.4 \mathrm{meV}$. Such a value would change the emission of the well by an easy-to-measure value of $20 \mathrm{~nm}$. This estimation assumes the perfect addition of a single monolayer to the quantum well, which is unrealistic but provides a useful approximation. For higher accuracy, atomistic modelling has been employed to explore the effects of local alloy, wellwidth, strain and built-in field fluctuations and it has been found that the variation is much greater than this, with a spread in emission energies of approximately $125 \mathrm{meV}^{19}$.

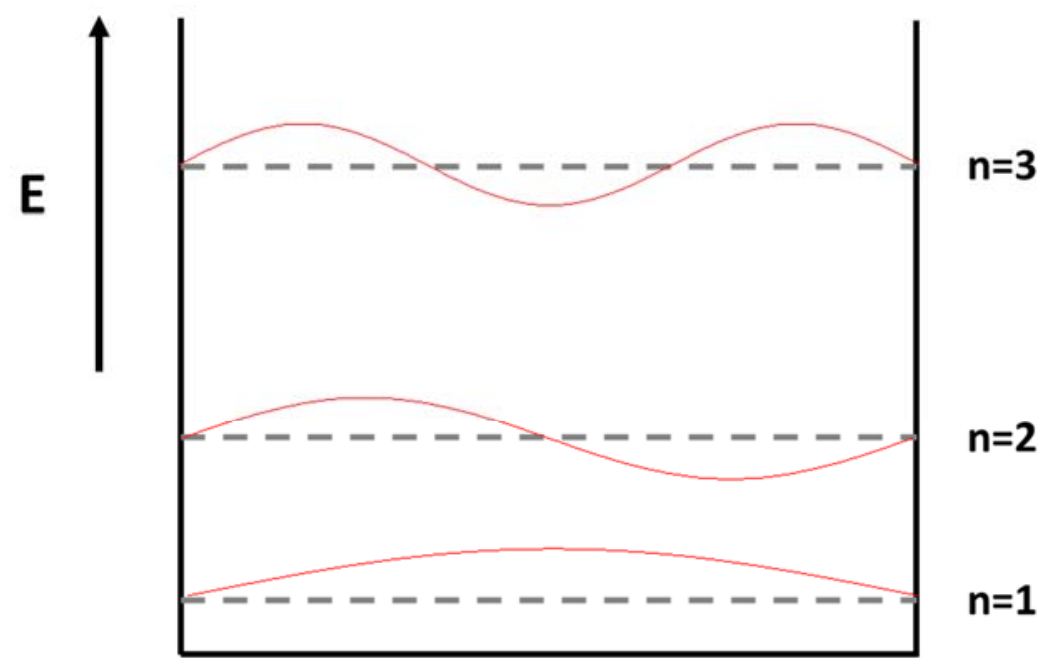

Figure 2.8: The wavefunctions of the first three energy levels, derived from an infinite potential well and the particle in a box assumption.

The eigenenergy of the quantum well can also be tuned by purposely changing the width of the well, leading to a simple methodology of designing a material system to display ideal properties; tuning it to emit in the infra-red, for example. In a quantum well, however, the electrons and holes are only confined in one dimension with a continuum of states still being allowed in the two directions perpendicular to the well. An interesting consequence of this confinement is that the electron and hole's wavefunctions have an increased overlap which results in an increased probability for radiative recombination; as predicted by Fermi's golden rule ${ }^{18}$. However, in reality the well height will be finite which leads to the quantum tunneling of these wavefunctions into the barriers. 
From fig 2.8 , it can be seen that the first confined energy level lies above the band-edge. This is an interesting observation and is known as the zero-point energy or the confinement energy. It arises as a result of the Heisenberg uncertainty principle, and is explained via the following. As the particle becomes constrained to a finite region of space, the variation in its position reaches an upper limit. As such, the particles momentum cannot be zero and it must possess some finite energy to ensure it does not violate the uncertainty principle. This energy varies with the well-width as more confinement results in less variability in space, so a higher zero-point energy for the particle is observed.

\subsubsection{Beyond 1 Dimension of Confinement}

The discussion presented above for quantum wells considered the confinement of electrons and holes in 1 dimension, where the continuum of states in the two perpendicular directions still existed. Using similar epitaxial and lithography techniques, nanostructures that can confine electrons and holes in 2 and 3 dimensions can also be fabricated. Such structures are known as a quantum wire (QWi) and a quantum dot (QD), respectively ${ }^{18}$.

A common way to characterise a given quantum structure is the density of states, which provides information about the number of states available at a given energy level. The density of states for 0 (bulk), 1 (QW), 2 (QWi) and 3 (QD) dimensions of confinement is illustrated in fig 2.9. The eigenfunctions and eigenenergies of each of these systems is quantitatively different, but in general the following effects are seen:

1. With higher dimensional confinement, more blue-shift is seen in the band-edge of the semiconductor, due to the Heisenberg uncertainty principle criteria mentioned above.

2. As the electrons and holes are confined into a smaller region of space, the wavefunction overlap continues to increase, so the radiative recombination probability also increases.

3. The density of states displays higher discretisation as the particles have less freedom of movement, with the QD eventually showing fully discrete energy levels, similar to that of atoms; for this reason QDs are sometimes called 'artificial atoms'. 


\subsubsection{Excitons}

As mentioned previously, an exciton is a quasiparticle consisting of a bound electron-hole pair. These particles are formed in bulk semiconductors when an electron is excited across the band gap and the Coulomb interaction holds the particles together. These quasiparticles are conceptually similar to a Hydrogen atom, and also display discrete energies. The main difference is the closely matched effective masses of the electron and hole, as opposed to the Hydrogen atom which consists of a proton and an electron. However, in the bulk, the thermal energy at room temperature is enough to rip the particle apart such that the effects on the properties of the system become negligible. On the other hand, in a nanostructure exhibiting quantum confinement, the effects of the exciton become highly important, even at room temperature.

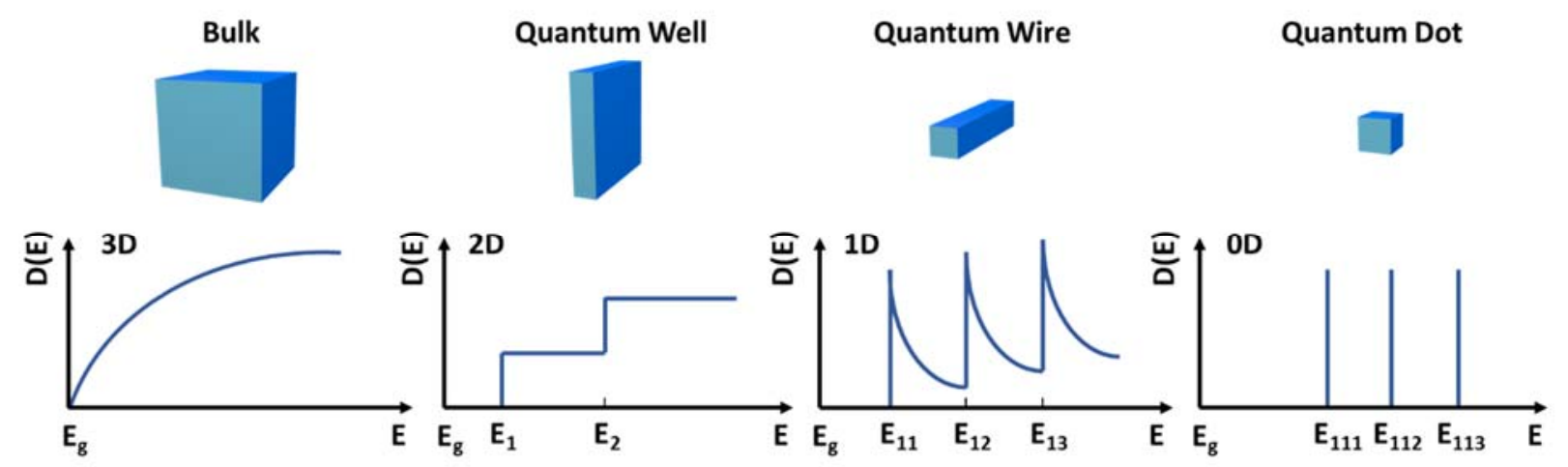

Figure 2.9: The density of states for a bulk semiconductor, a quantum well, a quantum wire and a quantum dot, showing the increasing amount of discretisation in the system. The subscripts denoting the energy levels correspond to the principal quantum numbers in the $\mathrm{x}, \mathrm{y}$ and $\mathrm{z}$ direction.

Due to their similarities, the same equations that describe the Hydrogen atom can be applied to an exciton. This approach gives a binding energy and Bohr radius of the exciton as,

$$
E_{B}=\frac{\mu e^{4}}{8 h^{2} \varepsilon_{r}^{2} \varepsilon_{0}^{2}} \quad a_{e x}=\frac{\varepsilon_{e} m_{0} a_{0}}{\mu}
$$

where $\mu$ is the reduced mass of the electron-hole system $\left(\frac{m_{e}{ }^{*} m_{h}{ }^{*}}{m_{e}{ }^{*}+m_{h}{ }^{*}}\right), \varepsilon_{\mathrm{r}}$ is the dielectric constant, $\varepsilon_{0}$ is the permittivity of free space, $e$ is the charge of an electron and $a_{0}$ is the Bohr radius of the Hydrogen atom (0.52 $\AA$ ). In semiconductors, two types of excitons can exist, Frenkel and Wannier-Mott excitons. 
These correspond to the radius being comparable to the size of the unit cell and much larger than the unit cell, respectively. In semiconductors, simple calculations show that the excitons are of the Wannier-Mott variety, with a radius of $\sim 300 \AA$ in GaAs (with a unit cell of $5.65 \AA$ ).

Quantum confinement effects can change the properties of excitons. For example, when an electronhole pair is created in a quantum well, it has a smaller radius governed by the size of the well. This results in the exciton having an increased binding energy which makes it more difficult for phonons to destroy the particle. Likewise, the closer proximity increases the absorption strength due to the larger electron-hole overlap. These effects mean that the excitons present in a quantum nanostructure are able to be resolved at room temperature, which has great consequences as these features can then be used in practical applications.

\subsection{Aim of This Work: The Sensitivity to Imperfections}

The atomic structure and composition of such semiconductor nanostructures can play a vital role in affecting the resulting qualities of the system. Amazingly, in some semiconducting systems, the alteration of a single atom can have profound consequences on the properties ${ }^{20}$. In the majority of applications, it is of great interest to completely remove these imperfections that can arise through vacancies, interstitial atoms, interfacial defects and grain boundaries, amongst others ${ }^{21}$. The existence of imperfections in nanostructures of the three degrees of confinement have been studied, with negative implications on the resulting properties of the system $22,23,24,25$.

In this work, it is suggested to use these imperfections that are seen by many as faulty, for a novel application in information security. By employing quantum nanostructures as UNO/PUF devices, the smallest possible unit for a unique identity is proposed, which derives its fingerprint from the unique arrangement of atoms. These systems would be more difficult to clone than any other existing system, as it would require the measurement and re-fabrication of a structure on the atomic-scale. Furthermore, due to its extreme size, it would provide the largest possible secure bit density, allowing the devices to be employed with a low footprint into limited-power systems. The foundations for 
unique identities based on electronic, optical and optoelectronic systems have been laid by employing various semiconducting structures that exhibit quantum confinement. These three types of system would provide a large bandwidth, capable of employing such systems into a variety of devices and applications that require unique identities.

\subsection{Thesis Outline}

In chapters 1 and 2, the background and motivation for creating unique identification devices with more robust security has been provided, whilst exploring some of the fundamental properties that define semiconductor nanostructures. It is difficult to give a comprehensive overview of all the properties that can be affected in a semiconductor crystal, and an interested reader is asked to refer to dedicated texts on the topic ${ }^{7,18}$.

The next chapter discusses the experimental methods and fabrication techniques that have been employed in the creation and characterisation of the devices. Specifically, the Langmuir-Blodgett technique coupled to an electrospray setup is discussed in detail.

The first experimental chapter, 'chapter 4', begins by discussing the common defects and imperfections found in semiconductor crystals, followed by the inspection of a quantum dot system which displays unique photoluminescence spectra due to the unique structure and composition of each dot. Next, a device that allows a simple electronic measurement of quantum phenomena at room temperature is introduced; the Resonant Tunneling Diode (RTD). The RTD comprises a double-barrier quantum well structure that utilises quantum tunneling, and is characterised by the presence of a peak in the devices current-voltage characteristic and a region of negative differential resistance. The fine tuning of the current-voltage is spectra is then explored by manipulating the measurement protocol such that the spectra from a given device is as steady and reliable as possible. Following the definition of these characteristics, a number of devices with identical geometries are probed and the resulting current-voltage spectra are analysed. It is found that the peak in the current-voltage spectra, which highlights the internal energy level, is unique for 26 identical devices with a $99.997 \%$ confidence. 
The repeatability of such a measurement is then explored by taking 100 measurements of a number of devices. It is found that the repeated measurement of an RTD produces very robust current-voltage characteristics, where the peak of each measurement lies within 2 standard errors of the average value.

In the second experimental chapter, 'chapter 5', the creation of an optical analogue to an electronic unique identifier is discussed. In the work presented here, 2D materials were chosen as an ideal material system capable of producing thin-transparent films that can emit light once excited. The chapter begins with a brief overview of the properties of the most popular 2D material, graphene, followed by an introduction to the transition metal dichalcogenides (TMDs), specifically $\mathrm{MoS}_{2}$. The fabrication techniques of such materials is discussed and in this work, the creation of thin films of $\mathrm{MoS}_{2}$ by the Langmuir-Blodgett technique is suggested for the first time. It was found that thin films of $\mathrm{MoS}_{2}$ could be fabricated by this technique by employing electrospray to deposit the material on an air-water interface. Optical identification tags were then created using this technique but it was found that the thin film displayed no photoluminescence. To investigate the limiting factors, a variety of methods were employed on the film and it was found that the underlying cause was the thickness of the film, $7.6 \pm 0.6 \mathrm{~nm}$, which corresponds to 12 layers. Finally, different starting solutions were employed which contained high monolayer contents, and subsequently emitted once deposited.

The final experimental chapter, 'chapter 6', takes the Langmuir-Blodgett technique one step further by assisting in the creation of graphene/nanoparticle/graphene heterostructures, to create coupled 2D/OD systems via this technique, for the first time. First, a brief background into semiconducting nanoparticles is discussed, with a focus on CdSe nanoparticles. The particular system used in this work utilises silica capped CdSe nanoparticles, which have received little attention, but are advantageous due to their ability to be suspended in non-toxic chemicals, which would provide a much greater integration potential. It was found that the nanoparticles could be deposited both on to $\mathrm{SiO}_{2}$ and graphene with coverage percentages of $63.55 \pm 3.20 \%$ and $64.83 \pm 2.75 \%$, respectively. The average 
height of the film and diameter of the nanoparticles was found to be $38.4 \pm 2.4 \mathrm{~nm}$ and $30.5 \pm 0.3 \mathrm{~nm}$, respectively, in agreement with predictions and indicates no degradation of the nanoparticles. Conductivity measurements on the heterostructures highlighted that there were short-circuits in the gaps between nanoparticles. Finally, to achieve visible fluorescence, the subphase was changed from water to dimethyl sulfoxide, which also resulted in a more tightly packed film that should result in the removal of short-circuits.

In chapter 7, the work in the thesis is concluded and the suggestions of some future research directions is discussed. 


\section{Bibliography}

${ }^{1}$ M. Cardona \& F. H. Pollak, "Energy-band Structure of Germanium and Silicon: The k.p Method", Phys. Rev., 142,530 (1966).

${ }^{2}$ H. Ehrenreich, "Band Structure and Electron Transport of GaAs", Phys. Rev., 120, 1951 (1960).

${ }^{3}$ J. F. Prins, "Fermi-Dirac Statistics and the Nature of the Compensating Donors in Boron-doped Diamond Layers", Phys. Rev. B., 39, 3764 (1989).

${ }^{4}$ G. H. Wannier, "On the Energy Band Structure of Insulators", Phys. Rev., 76, 438 (1949).

${ }^{5}$ C. S. Hung \& V. A. Johnson, "Resistivity of Semiconductors Containing both Acceptors and Donors", Phys. Rev., 79, 535 (1950).

${ }^{6}$ L. P. Bouckaert et al., "Theory of Brillouin Zones and Symmetry Properties of Wave Functions in Crystals", Phys. Rev., 50, 58 (1936).

7 “Fundamentals of Semiconductors", P. Y. Yu \& M. Cardona, Springer, Berlin (2010).

${ }^{8}$ G. H. Parker \& C. A. Mead, “Energy-Momentum Relationship of InAs”, Phys. Rev. Lett., 21, 605 (1968).

${ }^{9}$ W. G. Spitzer \& H. Y. Fan, “Determination of Optical Constants and Carrier Effective Mass of Semiconductors", Phys. Rev., 106, 882 (1957).

${ }^{10}$ R. N. Dexter \& B. Lax, “Effective Masses of Holes in Silicon”, Phys. Rev., 96, 223 (1954).

${ }^{11}$ M. D. Sturge, "Optical Absorption of Gallium Arsenide Between 0.6 and 2.75eV", Phys. Rev., 127, 3 (1962).

${ }^{12}$ M. A. Green \& M. J. Keevers, "Optical Properties of Intrinsic Silicon at 300K", Prog. In Photovoltaics, 3, 3 (1995).

${ }^{13}$ P. T. Landsberg \& M. J. Adams, "Radiative and Auger Processes in Semiconductors", Journal of Luminescence, 7, 3 (1973).

${ }^{14}$ D. J. Fitzgerald \& A. S. Grove, "Surface Recombination in Semiconductors", Electron Devices Meeting (1967).

${ }^{15}$ R. Hill, “Energy-gap Variations in Semiconductor Alloys”, J. Phys. C: Solid State Phys., 7, 521 (1974).

${ }^{16}$ C. B. Roxlo et al., "Evidence for Lattice-Mismatch-Induced Defects in Amorphous Semiconductor Heterostructures", Phys. Rev. Lett., 52, 1994 (1984).

${ }^{17}$ G. Scamarcio et al., "Optical and Vibrational Properties of $\mathrm{Al}_{x} \mathrm{Ga}_{1-x} \mathrm{As} / \mathrm{AlAs}$ Multiple Quantum Wells Near the Direct-indirect Cross-over", Surface Science, 228, 1 (1990).

18 "Optical Properties of Solids", M. Fox, Oxford University Press (2010).

${ }^{19} \mathrm{~S}$. Schulz et al., "Atomistic Analysis of the Impact of Alloy and Well-width Fluctuations on the Electronic and Optical Properties of InGaN/GaN Quantum Wells", Phys. Rev. B., 91, 035439 (2015).

${ }^{20}$ A. Gruber, "Scanning Confocal Optical Microscopy and Magnetic Resonance on Single Defect Centres", Science, 276, 5321 (1997).

${ }^{21}$ H. J. Queisser \& E. E. Haller, "Defects in Semiconductors: Some Fatal, Some Vital”, Science, 281, 5379 (1998).

${ }^{22} \mathrm{X}$. Zhou et al., "Observation of Subsurface Monolayer Thickness Fluctuations in InGaN/GaN Quantum Wells by Scanning Capacitance Microscopy and Spectroscopy", Appl. Phys. Lett., 85, 407 (2004). 
${ }^{23}$ M. Belloeil et al., "Quantum Dot Like Behaviour of Compositional Fluctuations in AIGaN Nanowires", Nano. Lett., 16, 2 (2016).

${ }^{24}$ R. Bao et al., "Conductance Fluctuations in Chaotic Bilayer Graphene Quantum Dots", Phys. Rev. E., 92, 012918 (2015).

${ }^{25}$ S. K. Kirby et al., "Atomic-scale Imperfections and Fluctuations in the Transmission Properties of a Quantum Dot", Phys. Rev. B., 50, 10990 (1994). 


\section{Chapter 3}

\section{Sample Preparation and Experimental Techniques}

\subsection{Introduction}

In this chapter, the techniques that have been employed for the following experimental chapters will be discussed. The resonant tunneling diodes (RTDs) studied in chapter 4 were fabricated elsewhere, with the novel step in this work being in their application as a unique identifier. However, as they are relevant to the thesis, a summary of their fabrication is included in this chapter with the appropriate reference provided to direct the reader to the work dedicated to their manufacture. To utilise RTDs as an efficient tool for unique identification, electronic measurements were taken in this work using a probe station, to allow simple and efficient measurement of a large array of devices. In this chapter, the principal behind the electronic measurements of the RTDs is introduced with the appropriate parameters that can be optimised to give the optimum repeatability of results, which are then applied to all subsequent measurements of RTDs. The majority of the chapter focusses on the LangmuirBlodgett (LB) technique used to fabricate the devices studied in chapters 5 and 6 . In this work, the major restriction of the LB technique is addressed. This bottleneck is the lack of ability to spread a given molecule or nanomaterial suspended in solution onto the surface of a liquid-air interface using conventional methods. This is due to the common miscibility of the two liquids, which causes ample mixing when the nanomaterial suspended in solution is dropped onto the surface, leaving very little material at the interface. To circumnavigate this problem, an electrospray method is applied to nebulise the solution which reduces the volume of liquid droplets by approximately three orders of magnitudes and significantly reduces mixing. Using these techniques, the deposition of thin films of $\mathrm{MoS}_{2}$ (chapter 5) and silica-capped nanoparticles (chapter 6) is achieved for the first time. Finally, the 
methods used for the characterisation of the fabricated thin films will be introduced and discussed, including; scanning electron microscopy (SEM), a number of scanning probe microscopies (SPM), and Raman, micro-photoluminescence and fluorescence spectroscopies.

\subsection{Production of Resonant Tunneling Diodes}

\subsubsection{Molecular Beam Epitaxy Growth}

The RTDs MBE growth procedure can be found in previous work ${ }^{1}$, and its following description is given only for completeness. The target substrate was semi-insulating indium phosphide with a (100) crystal orientation. The growth is summarised as follows. Firstly, a $400 \mathrm{~nm}$ collector-contact layer of $\ln _{0.53} \mathrm{Ga}_{0.47}$ As was grown with an $\mathrm{n}+$ doping concentration of $1.0 \times 10^{19} \mathrm{~cm}^{-3}$, followed by a $25 \mathrm{~nm}$ collector layer of the same material but with an $\mathrm{n}+$ doping concentration of $3.0 \times 10^{18} \mathrm{~cm}^{-3}$. Next, a spacer layer with $20 \mathrm{~nm}$ width was grown with the same composition as above. The spacer layer prevents diffusion of dopants into the layers above this step. To define the quantum well, two $1.2 \mathrm{~nm}$, undoped AlAs barriers were grown that sandwiched a highly strained, undoped $\ln _{0.8} G_{0.2} A s$ layer of width $4.5 \mathrm{~nm}$. To finalise the structure, an undoped $20 \mathrm{~nm}$ spacer layer, $25 \mathrm{~nm}$ emitter layer and 45 $\mathrm{nm}$ emitter-contact layer consisting of $\mathrm{In}_{0.53} \mathrm{Ga}_{0.47} \mathrm{As}$ with doping concentrations of $3.0 \times 10^{18} \mathrm{~cm}^{-3}$ and $2.0 \times 10^{19} \mathrm{~cm}^{-3}$ (for the latter two), respectively, was grown. The highly doped emitter-contact layer was grown to enable good ohmic contacts.

\subsubsection{Device Fabrication}

The fabrication of RTDs was also provided in ref [1]. Here, a top contact-layer was defined via conventional optical lithography before thermal evaporation of $50 \mathrm{~nm}$ of titanium and $250 \mathrm{~nm}$ of gold defined the ohmic contact layer. This metal contact also acts as the mask for the subsequent etching steps. A reactive-ion etching process was employed via the use of a mixture of $\mathrm{CH}_{4}$ and $\mathrm{H}_{2}$, which gave an etch rate of $21 \mathrm{~nm} / \mathrm{min}$ and facilitated anisotropic side-walls all the way to the bottom contact layer. Before the bottom contacts were defined, an orthophosphoric-based wet-etch process was used with a ratio given as $\mathrm{H}_{2} \mathrm{O}: \mathrm{H}_{3} \mathrm{PO}_{4}: \mathrm{H}_{2} \mathrm{O}_{2}=50: 3: 1$. The etch rate here was $90 \mathrm{~nm} / \mathrm{min}$ and allowed 
separate devices to be isolated by etching down to the InP substrate; the wet-etch also provided an undercut which provided a means to create the air-bridges in the RTDs. Finally, a bottom contact layer of $50 \mathrm{~nm}$ of titanium and $500 \mathrm{~nm}$ of gold was deposited.

\subsubsection{Probe Station Measurements}

All the measurements of RTDs in chapter 4 were performed at 300K using a Wentworth Laboratories Ltd. SPM197 probe station connected to two $1.25^{\prime \prime}$ tungsten probes with tip radii of $1 \mu \mathrm{m}^{2}$. The basic circuit diagram for the measurement of RTDs is shown in fig 3.1. Here, a 2-wire sensing measurement (as opposed to 4-wire) is sufficient as the current flowing through a typical RTD is on the order of mA, which results in negligible errors that can be contributed by the testing leads if their voltage drop is large (large currents). For this assumption to hold, the testing leads must have a low resistance value, no greater than $1 \Omega$. This can easily be confirmed for copper wires of $1 \mathrm{~m}$ length and $0.1 \mathrm{~mm}$ radius, with a room temperature resistivity of $1.68 \times 10^{-8} \Omega \mathrm{m}$; this gives a lead resistance of $0.5 \Omega$.

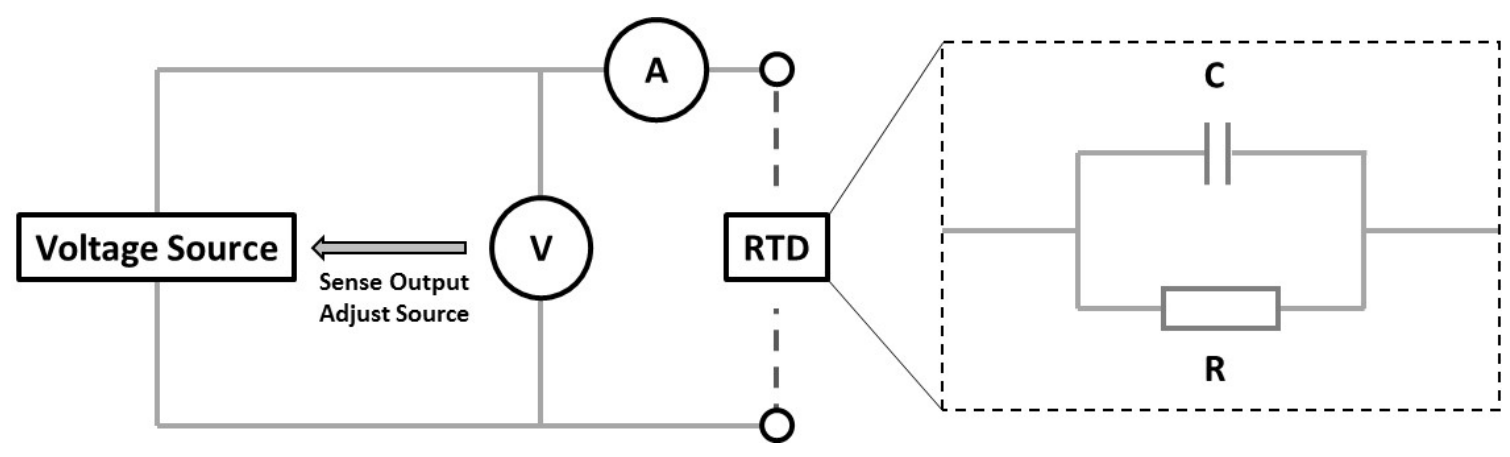

Figure 3.1: 2 wire sensing measurement of the RTDs used in this study. A voltage is applied to the RTD, and the current is measured. The voltage is sensed at the terminals of the SMU and used to adjust the source value. The RTD is normally considered as a parallel capacitor-resistor component.

In all measurements, the voltage was used as the input source and the current flowing through the RTD was measured. For greater accuracy, sense circuitry is used to monitor the output of the source and make adjustments if necessary. Here, the voltmeter senses the voltage at the output terminals and compares it to the programmed voltage level. If a discrepancy is measured, it adjusts the value of the voltage source accordingly. This mechanism helps to eliminate any voltage drops in the test leads 
by ensuring that the exact programmed voltage is present at the RTD.

Fig $3.2 \mathrm{~b}$ shows the typical operation (known as a source-delay-measurement (SDM) cycle) of the source measurement unit used during data acquisition (Keithley 2400 unit $^{3}$ ) and is summarised as follows. Once the source is turned on, there is a period of trigger latency that occurs when the source output makes a transition from off to on. Specifically, the trigger latency is the time taken to set the voltage or current to the programmed initial source value and is fixed at $100 \mu \mathrm{s}$. As long as the source output stays on, the trigger latency is not included in subsequent SDM cycles.

(a)

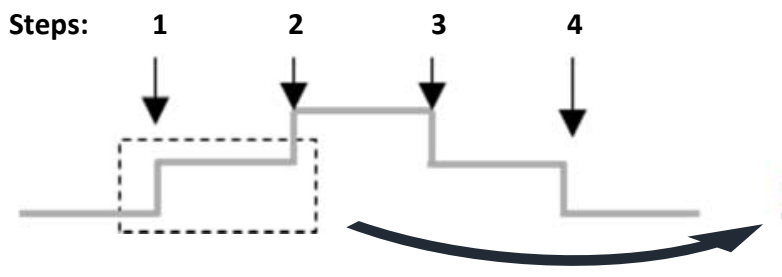

(b)

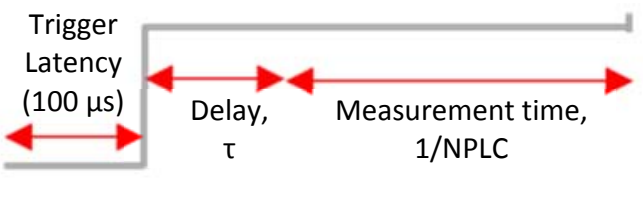

Figure 3.2: Source-delay-measurement (SDM) cycle during a typical measurement, showing (a) a complete sweep and (b) a single cycle. A typical step in the cycle involves a delay time, $\tau$, that allows the source value to settle, before the measurement is integrated over a time defined by the NPLC value.

Following the trigger latency, a delay time, $\tau$, is used which allows the source to settle at the value which is input before a measurement is performed. This value can be tuned between 0 and $9999 \mathrm{~s}$, as often longer times are needed to settle the external circuitry. For example, loads that are high in capacitance require a much larger settling time for the source. The optimum delay time needed for an accurate measurement can be calculated, or determined by trial-and-error. In this work, trial-anderror was used due to the complex nature of the calculation required for the time constant of the RTD (which can be modelled as a parallel resistor-capacitor configuration, as illustrated in fig 3.1). Once the source has been allowed adequate time to settle, the measurement time is defined by the measurement speed, given by the power lines cycle, NPLC, of the system. This value corresponds to the integration time over which the measurement is taken and then averaged. This measurement speed ranges from 0.01 to 10 , and corresponds to a measurement time of $0.01 / f-10 / f$, where $f$ is the 
power line frequency, $50 \mathrm{~Hz}$. Therefore, the measurement time can be varied between $0.2-200 \mathrm{~ms}$. This SDM cycle is then repeated over a number of steps that can be defined by the number of points in the sweep. In this work, a linear staircase sweep was used which increases the source value by an equal amount during each step, as illustrated in fig 3.2a. Furthermore, the final source value was always programmed so that it swept back to zero to prevent large voltage changes occurring between the device terminals. The effect of changing the delay and measurement times for RTDs is explored in chapter 4.

A number of other parameters have to be set when measuring the RTDs, such as the number of points in the sweep and the current compliance. The number of points is also explored in chapter 4 . The current compliance is extracted for the different sizes of RTDs by slowly increasing the compliance from a low value ( $0.5 \mathrm{~mA})$ until the peak in the RTD spectra is seen, if the compliance is set much higher than this, critical device failure is observed due to thermal degradation.

This section has concerned the measurements of RTDs, a semiconducting system based on III-V alloys. This is an ideal system for the simple yet robust electronic measurements required for unique identification. In the next section, a method to create a semiconducting system that can behave as an optical analogue of a unique identification system is introduced. Specifically, this concerns the fabrication of macroscopic thin films of material via a facile method known as the Langmuir-Blodgett technique.

\subsection{The Langmuir-Blodgett Technique}

\subsubsection{Background}

In this section, the techniques that have been used to overcome one of the longstanding problems with LB deposition, the strict requirements of using specific solvents, are discussed. Here, the use of electrospray for the spreading of material onto an air-water interface has been introduced to circumnavigate the problem of mixing. To this end, the deposition of $\mathrm{MoS}_{2}$ and silica-capped nanoparticles, nanomaterials that have to date not been fabricated via the LB technique due to this 
problem, is successfully achieved. This technique has also been employed for the construction of novel double heterostructures for optoelectronic applications; whilst it is straightforward to increase the complexity of such heterostructures by sequentially depositing selected materials. Moreover, different subphases that prevent the photoluminescence quenching of nanoparticles have been explored and were shown to successfully improve the deposition coverage, whilst eliminating the quenching.

The creation of thin films with a thickness of a single monolayer holds great promise in a number of applications such as the creation of next-generation sensors, displays and electronic circuit components. The Langmuir-Blodgett technique holds specific promise as it allows for the precise control of monolayer thicknesses of thin films and facilitates the homogenous deposition of material over extremely large areas. Furthermore, it can be implemented to create multi-layer structures of varying composition whilst allowing the transfer of monolayers onto effectively any substrate.

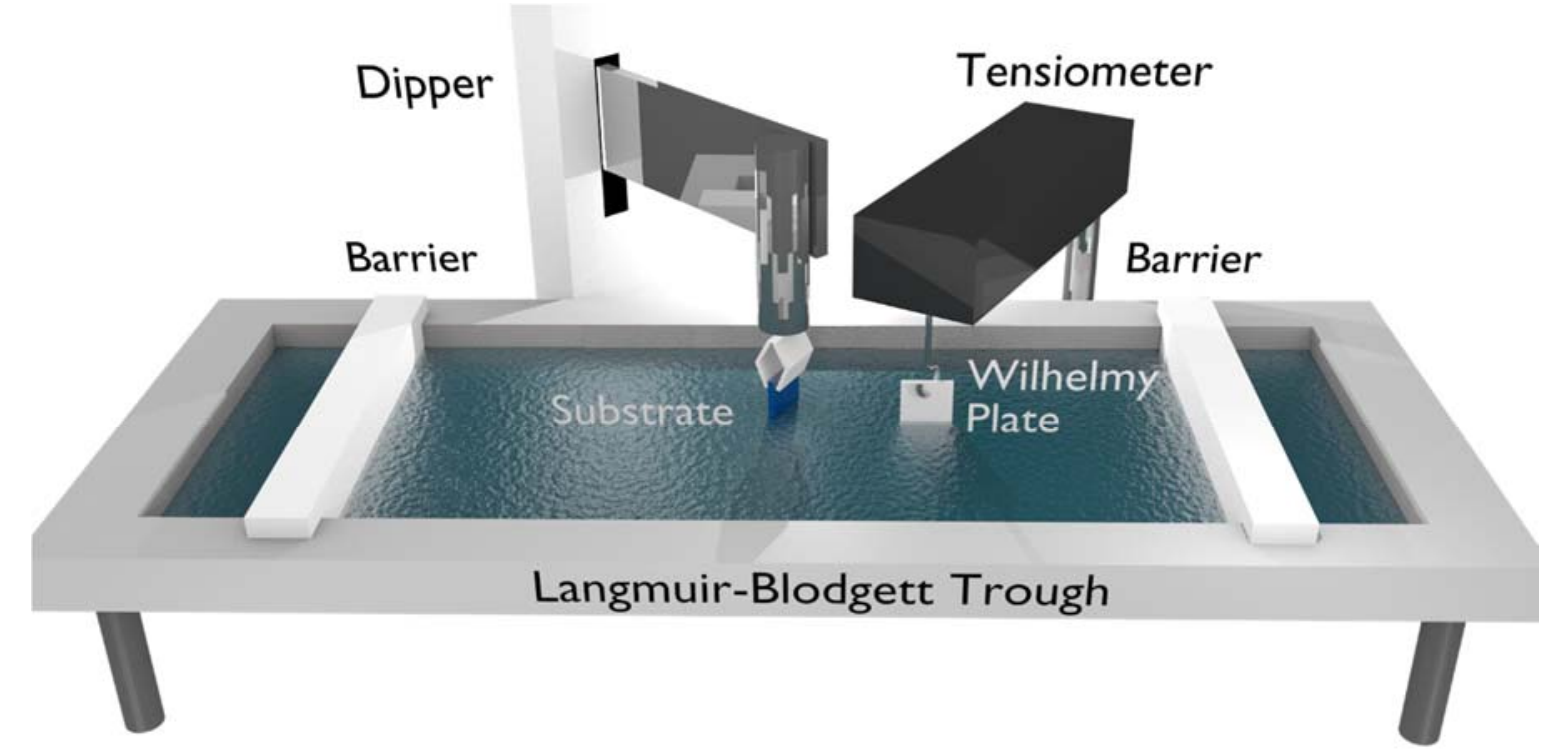

Figure 3.3: Experimental illustration of the major components of an LB trough. A water surface is contained by two moveable barriers that control the surface pressure of a material on the surface. The surface pressure is measured with a Wilhelmy plate (described later) attached to a tensiometer. When the desired pressure is reached, the dipper moves a substrate through the surface to deposit the material. 
The history of Langmuir-Blodgett films can effectively be dated back all the way to the 1700 's, when Benjamin Franklin reported his observations about how a teaspoon of oil on water spread to an area of about half an acre. Should he have made some quantitative measurements, he would have found that the oil had actually formed a monolayer. It was not until over a century later that Lord Rayleigh quantified this, and calculated that the thickness of this film would have been $1.6 \mathrm{~nm}$. Irving Langmuir was the first person to extensively characterise floating monolayers on a water surface in 1917, which subsequently won him the Nobel Prize ${ }^{4}$. To this end, he reported the transfer of molecular monolayers of fatty acids onto solid substrates. The first detailed description of sequential monolayer transfer was not until 1935 by Katherine Blodgett ${ }^{5}$. Today, these built-up monolayers of material hence received the name Langmuir-Blodgett (LB) films. Whereas floating monolayers on the water surface are usually reserved the name of Langmuir films.

\subsubsection{The Langmuir-Blodgett Trough}

To create an LB film, molecules (commonly surfactants) are spread onto the surface of a liquid known as the subphase; typically, this is water. The air-water interface contains an excess free energy that originates from a difference in environments of the molecules on the surface and those in the bulk of the liquid. This excess free energy can be probed through measurements of the surface tension, $\curlyvee$. For water, this surface tension has a value of $73 \mathrm{mN} / \mathrm{m}$ at room temperature, which is extremely high compared to other liquids and makes water a good subphase.

The spreading of material is normally achieved by dissolution in a volatile, non-polar solvent such as chloroform and then employing a micro-syringe to carefully drop the solution onto the surface of the subphase. This solution then spreads across the surface and when evaporated, forms a monolayer of material. To control the density of this surface monolayer, moveable barriers that confine the water surface are employed and can be opened and closed to lower or raise the density of the system, respectively. The two-dimensional analogue of pressure, the surface pressure ( $\Pi$ ), is calculated by measuring the difference in surface tension with and without a monolayer present on the water 
surface; this is frequently achieved with a Wilhelmy plate whose operation is discussed later in the section. Finally, for the deposition of a monolayer, the barrier positions are controlled with a feedback system whilst a dipper arm pulls a target substrate out of the subphase at a given speed. An illustration of the experimental system with the previously ascribed components is shown in fig 3.3 , and all experiments carried out in chapters 5 and 6 were performed on a trough like this, namely a KSV NIMA KN2003 LB trough ${ }^{6}$.

The trough is made of Teflon ${ }^{\mathrm{TM}}$, a hydrophobic material, which prevents leakage of water over the edges and confines the water to the trough area. On the other hand, the barriers comprise of Delrin ${ }^{\circledR}$, a hydrophilic material, which prevents the monolayer on the surface escaping underneath the barriers. The measurement of surface pressure is, as aforementioned, facilitated by the use of a Wilhelmy plate and is demonstrated in fig 3.4. Here, the force acting downwards on the plate is given by,

$$
F=\rho_{p} g l w t+2 \gamma(w+t) \cos \emptyset-\rho_{l} g t w h
$$

where $\rho_{p}$ and $\rho_{l}$ are the densities of the plate and liquid, respectively, $\gamma$ is the surface tension, $g$ is 9.8 $\mathrm{ms}^{-2}$, and $\mathrm{l}, \mathrm{w}, \mathrm{t}, \mathrm{h}$ and $\Phi$ are all defined in fig 3.4. Rearranging for the surface tension, assuming a contact angle of $0^{\circ}$, and subsequently taking the difference between a surface with and without a monolayer yields the surface pressure of the monolayer:

$$
\Pi=\Delta \gamma=\frac{\Delta F}{2(w+t)}=\frac{\Delta F}{2 w}, \text { if } w \gg t
$$

This shows that by using thin plates, the surface pressure can easily be recorded by a tensiometer (see fig 3.3) which records the changes in the force acting on the plate. In all experiments used in the following chapters, disposable paper plates of width $10.3 \mathrm{~mm}$ were used for the Wilhelmy plate. Metal plates can also be used, but their cleanliness is easily degraded through adsorption of molecules.

\subsubsection{A Typical Isotherm Experiment}

In a typical isotherm experiment, the successful creation of a Langmuir film at the air-water interface 
is as follows. Firstly, the entire trough must be cleaned with an organic solvent such as chloroform or ethanol that does not degrade the Teflon ${ }^{\mathrm{TM}}$ that the trough is comprised of. Special attention must be paid to the barriers which are made of Delrin ${ }^{\circledR}$ and are unsuitable for cleaning with chloroform. A Wilhelmy plate is constructed by cutting an approximately $2 \mathrm{~cm}$ length piece of paper that has a predefined width of $10.3 \mathrm{~mm}$. A hole is then pierced into this paper plate at the centre of one end before

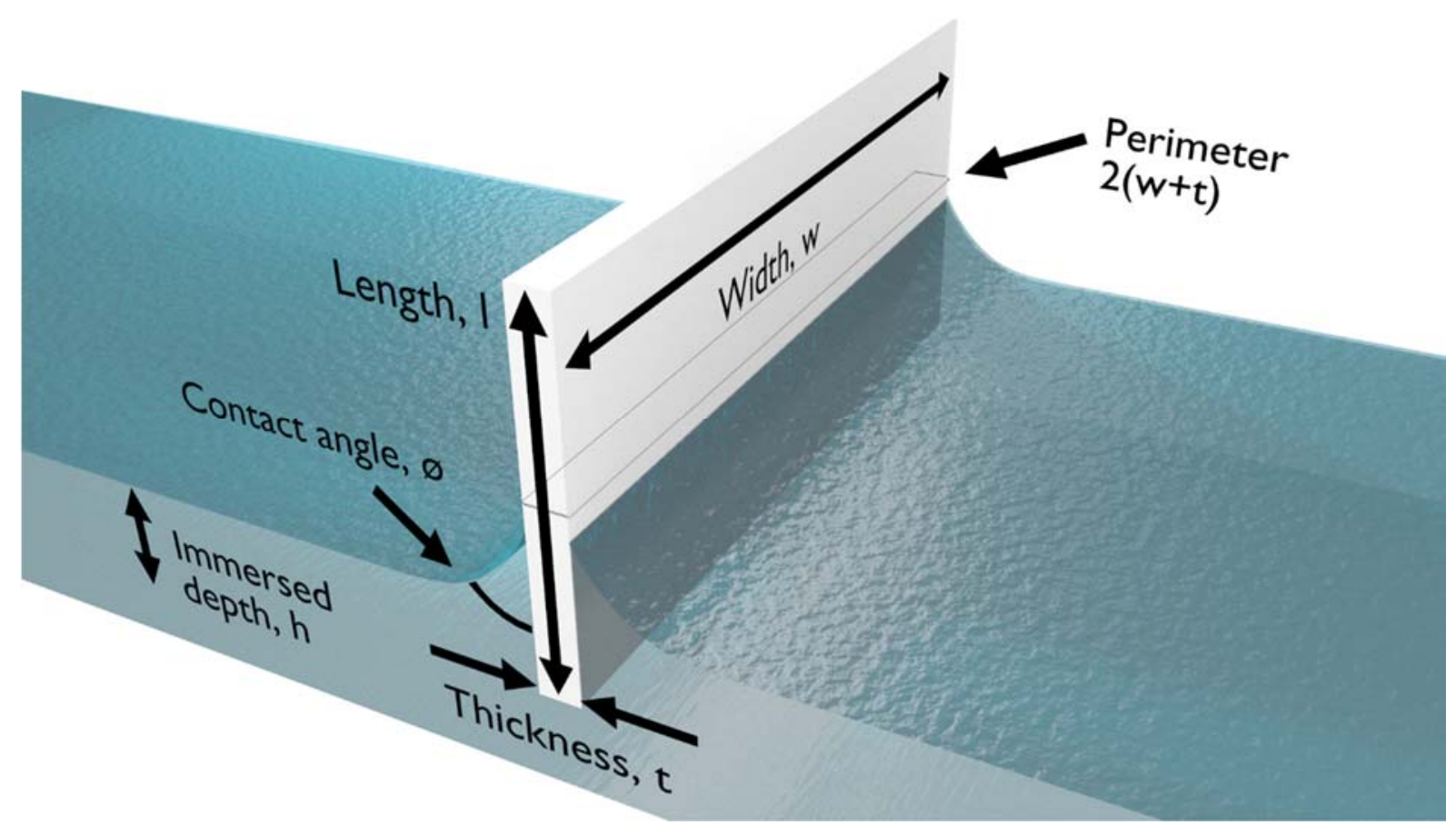

Figure 3.4: The Wilhelmy plate at the air-water interface, used to measure the surface pressure of the monolayer film. The contact angle, dimensions of the plate and immersed depth affect the force that acts on the film. However, to measure surface pressure, the important parameter is the perimeter and if a thin plate is used, the surface pressure can be calculated through the width of the plate alone.

being placed on the hook connected to the tensiometer. For best results, this Wilhelmy plate should be placed in a separate beaker of the same subphase for 30 minutes as this helps to clean the plate by dissolving any contaminants.

Subsequently, the entire trough is filled with deionised water such that the height of the meniscus rises above the levels of the barriers. One of the reasons a clean trough is required is to retain the hydrophobic/hydrophilic features of the trough/barriers; with the other being to prevent contamination. Once a continuous water meniscus is formed throughout the trough, the surface must 
be cleaned of oils, organics and other contaminants. To do this, an aspirator attached to a hose with a hydrophobic PTFE syringe on the end is used. The syringe is held close to the air-water interface such that a 'slurping' noise can be heard, this distance must be kept constant as if the syringe is placed too far into the water it will begin to drain the trough and allow leakage under the barriers. Whilst the surface between the barriers is being cleaned, the barriers are closed to their maximum position to allow all surface contaminants to be close-packed and removed. The barriers are then fully re-opened and the reading on the tensiometer's balance is zeroed. Following this, the barriers are closed in order to get a representation of the surface cleanliness. If the pressure rises above $0.3 \mathrm{mN} / \mathrm{m}$, the surface should be re-cleaned and this process repeated. After the surface is adequately clean, the barriers should be left in their open position to allow for the deposition of material. The solution is conventionally deposited onto the surface using a syringe, and following this the remaining solvent is allowed to evaporate so that only the molecules are present on the surface before an isotherm is taken.
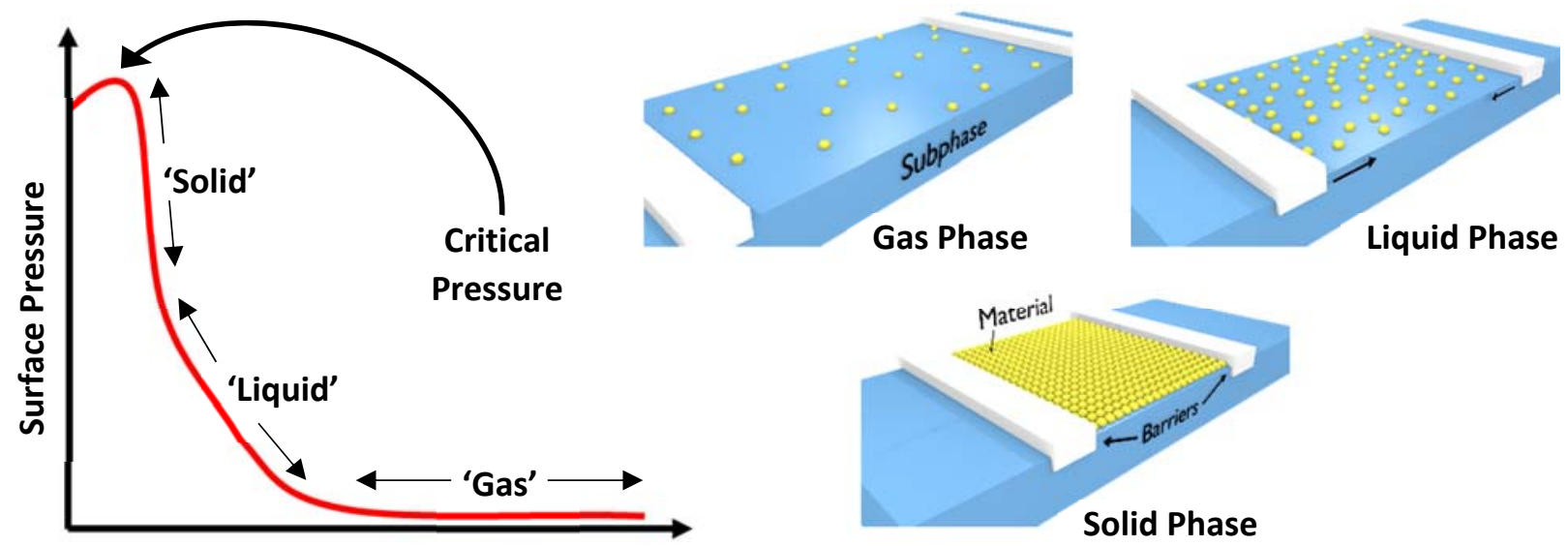

Surface Area

Figure 3.5: A typical pressure-area isotherm showing the three distinct phases normally seen during a compression cycle. When the molecules on the surface are sparsely spread, the molecules behave like an ideal 'gas' in a container (gas phase). As the area is reduced, a change in the gradient is observed indicating the formation of a 'liquid' due to non-negligible interactions between molecules (liquid phase). Further compression results in the creation of a 'solid' film (solid phase), which then collapses if the pressure is increased past the critical pressure. 
The most important indicator of the monolayer properties is given by measuring the pressure-area isotherm. This is achieved by reducing the area (closing the barriers) and constantly monitoring the pressure. A schematic of a typical pressure-area isotherm can be seen in fig 3.5. A number of distinct regions appear during compression and are described as follows. Before compression begins, and the barriers are extremely far apart, the molecules are allowed to move in a random motion on the surface and behave very similarly to an ideal 'gas' enclosed in a box. As the available area is reduced, the density of molecules on the surface increases as the 'gas' is compressed, corresponding to a small rise in pressure. At an area that is dictated by the material system, a phase transition similar to that of a conventional gas is seen as the molecules begin to interact and behave like a 'liquid'. Further compression results in the complete close-packing of the molecules and the formation of a 'solid' state; this solid film can then be transferred onto a substrate. If the pressure is increased past a 'critical pressure', the monolayer film becomes unstable and is destroyed due to the formation of threedimensional structures; this is commonly indicated by a sudden drop, or plateau, in pressure. The phase behaviour of the monolayer is mainly determined by the physical and chemical properties of the molecule in use, the subphase composition and the subphase temperature. Thus, the selection of the subphase is important for the resulting characteristics of the film. For example, it was found that the deposition of nanoparticles from a water subphase completely quenched the fluorescence. To this end, using dimethyl sulfoxide as the subphase facilitated LB films with excellent surface coverage whilst not diminishing the fluorescence of the nanoparticles. This gives the LB technique an extra variable that can be manipulated to achieve ideal deposition of a large variety of materials.

\subsubsection{The Mixing Problem}

In the Langmuir-Blodgett technique, the material to be deposited needs to disperse well both in an organic, water immiscible spreading solvent, such as chloroform or hexane, and on the surface of water. This is a major bottleneck for LB assembly as it is normally required for the dispersed material to have completely contrasting surface properties for dispersion in water or these solvents. This problem can be aggravated if the starting solution of the colloidal suspension is different to the 
(a)

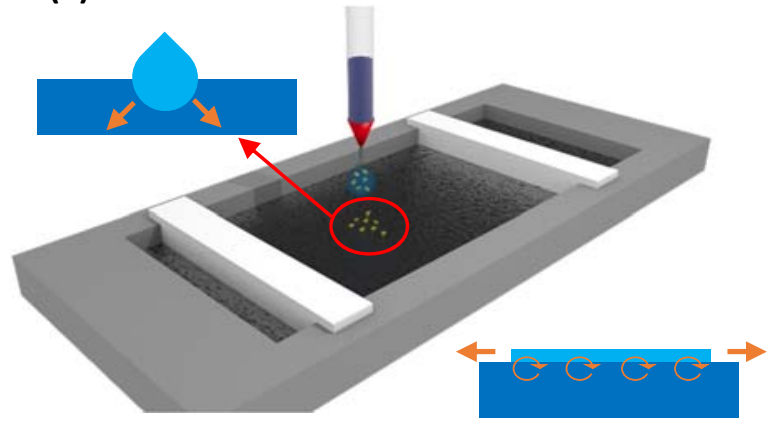

(c)

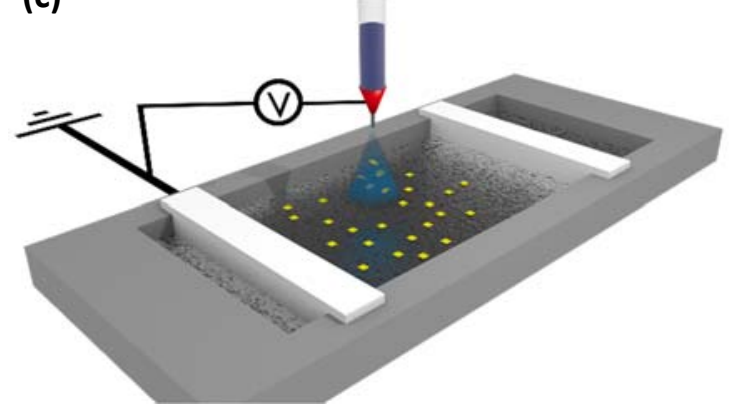

(b)

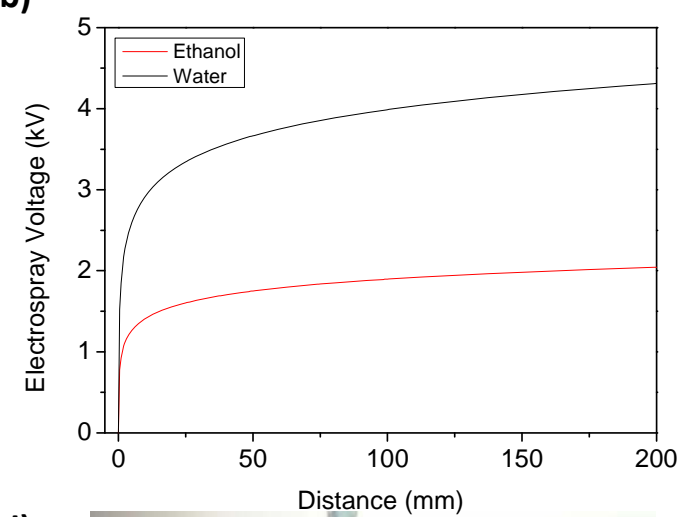

(d)

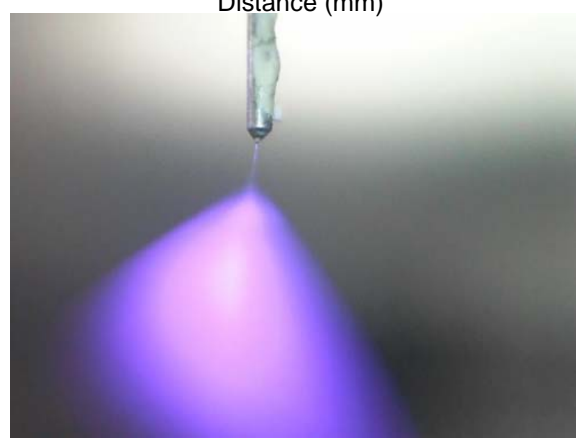

Figure 3.6: Electrospraying with the Langmuir-Blodgett technique. (a) A large amount of mixing during conventional spreading [Insets: direct mixing (top-left) and turbulent mixing (bottom-right), (b) Little mixing seen due to electrospraying onto the water surface, (c) 'On' voltage against distance from airwater interface surface for water and ethanol under experimental conditions, (d) Optical image of electrospraying ethanol in the cone-jet mode.

spreading solvent as the material must then be stabilised in a range of solvents. In order to mitigate this, alcohols have been used as the spreading solvent which, due to the surface tension mismatch with water, allow some spreading of the material. However, such alcohols can mix with water leading to the majority of material being lost to the subphase ${ }^{7}$. In conventional LB assembly using alcohols, the spreading of material is done via a syringe resulting in droplets approximately $\mathrm{mm}^{\prime} \mathrm{s}$ in size as demonstrated in fig 3.6a. The size of these droplets causes mixing into the water via two mechanisms. Firstly, upon impact of the droplet with the water surface, the alcohol directly mixes with the water due to partial submersion of the droplet (see fig 3.6a top-left inset). The other mixing mechanism is the result of the solvent spreading over the surface, this induces turbulent mixing below the surface which promotes mixing of the alcohol with water (fig 3.6 a bottom-right inset) ${ }^{8}$. Through the use of fine-control, such as releasing droplets by dragging them across the water surface, the direct mixing 
interaction can be largely reduced ${ }^{9}$. However, the effects of turbulent mixing cannot be alleviated unless a different dispensing method is employed.

\subsubsection{The Case for Electrospray}

Electrospray occurs when a liquid is directed through a capillary that is held at high electrostatic potentials. Such conditions can cause the liquid to spray and form aerosol particles, a phenomenon that was discovered by John Zeleny, coincidentally in the same year as Langmuir's seminal experiments ${ }^{10}$. There are a few main-modes that can be observed in the electrospray dynamics as the external electric field is increased. At low electric fields, a 'dripping-mode' is observed where droplets of liquid larger in diameter than the capillary drop at regular time intervals, commonly coupled with the formation of several satellite drops ${ }^{11}$. As the electric field is increased, a liquid meniscus forms at the opening of the capillary in a cone-like shape, with a jet appearing at the apex. This jet only remains for a short distance before breaking up into a 'brush spray' due to Coulomb fission. This mode was observed by Zeleny and analytically studied by Taylor, who calculated the equilibrium condition for the formation of the cone (when no jet was formed) and found that it was when the surface tension balanced the electric forces ${ }^{12}$. This mode is now known as the 'cone-jet mode'. Further increase of the electric field can lead to the formation of multiple cones and jets, known as the 'multi-jet mode'. The most common cone-jet mode is shown in fig 3.6d. A useful approximation for the voltage, $V_{O N}$, which gives the onset of electrospray, is,

$$
V_{O N}=2 x 10^{5}\left(\gamma r_{c}\right)^{\frac{1}{2}} \ln \left(\frac{4 d}{r_{c}}\right)
$$

where $\gamma$ is the surface tension of the subphase, $r_{c}$ is the radius of the dispensing needle and $d$ is the distance from the water surface. This is plotted in fig $3.6 \mathrm{~b}$ for water and ethanol (the two spreading solvents used in this work). Here, the surface tensions of water $(72 \mathrm{mN} / \mathrm{m})$ and ethanol $(22 \mathrm{mN} / \mathrm{m})$ at room temperature are used. Furthermore, the needle diameters employed for electrospraying were $100 \mu \mathrm{m}$ and $150 \mu \mathrm{m}$, for water and ethanol, respectively. Due its larger surface tension, water required 
a larger tip diameter to allow an adequate flow rate. Other factors can affect $\mathrm{V}_{\mathrm{ON}}$, such as the relative humidity and the composition of the needle, though the assumption applied here is that all other factors in the laboratory remained the same. Electrospraying the material results in the diameter of the droplets reducing to the nanometre to micron range and thus a reduction in volume of over 6 orders of magnitude, resulting in negligible direct or turbulent mixing. Spreading in this way allows material to remain at the water-air interface regardless of the spreading solvent, illustrated in fig 3.6c, and will be applied for all results in this work; in a way similar to that reported by Nie et al. ${ }^{13}$.

For electrospray of material used in this work, the exact conditions are given in the experimental chapters. An interesting point to mention is that no external apparatus was applied to dictate the flow rate of the system and the electric pressure was solely used to extract the solution. The flow rate is dependent on a number of variables including the applied voltage, capillary inner and outer diameter, capillary length and the capillary to surface distance, amongst others. The effects of changing these values can be found in ref. [14]. In brief, the flow rate increases linearly with the applied voltage and is dependent on the capillary geometry due to the changing electric pressure. The exception to this rule is the inner diameter of the capillary which changes the hydraulic impedance and can slow the flow rate. Due to this, the type of capillary that was used for the spraying of water and ethanol had to be changed, as aforementioned.

\subsection{Characterisation Techniques}

\subsubsection{Brewster Angle Microscopy (BAM)}

The Brewster angle microscope was employed in this study to allow the determination of the morphology and structure of the thin films that were present at the air-water interface. It provides an effective measurement of the presence of a film, which is often difficult to tell when using transparent solutions. This is an advantageous technique, as it allows the deposition of material to be discerned at an early stage (clarifying that minimal mixing is taking place), by direct inspection of the water surface. 
Through investigations of the polarisation properties of light reflected from transparent dielectric interfaces, David Brewster discovered that at certain angles, unpolarised light can be perfectly planepolarised ${ }^{15}$. Likewise, when the surface is illuminated with plane-polarised light at the same angle, all light will be transmitted into this medium with no reflection. This specific angle is now more commonly known as Brewster's angle.

The Brewster angle microscope makes use of this property by directing vertically polarised light at the Brewster angle of incidence towards an air-water interface. Through simple calculations using Snell's law and the refractive indices of the materials present, the Brewster angle can be found; for the airwater interface this angle is $53^{\circ}$.

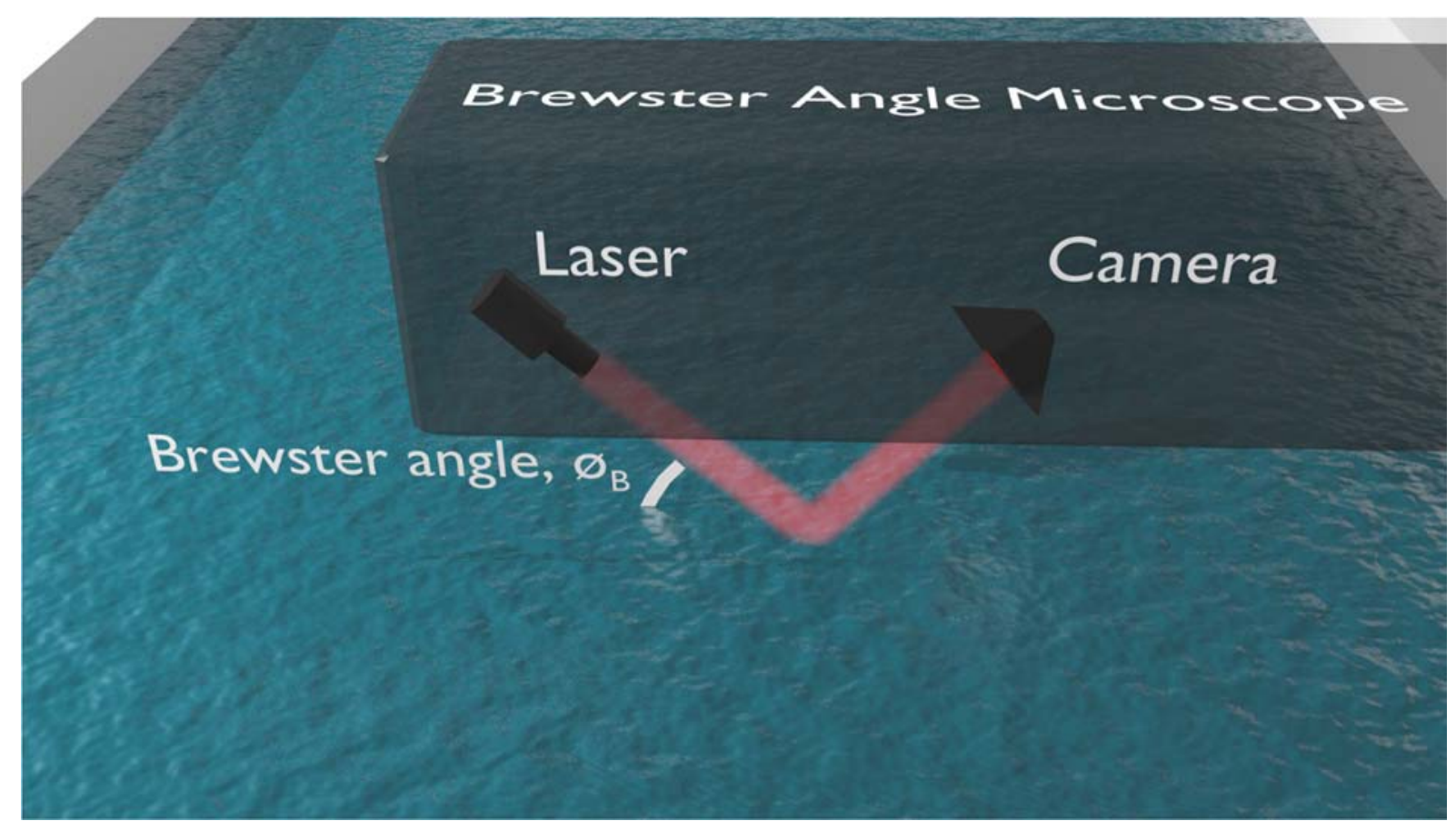

Figure 3.7: Brewster angle microscope experimental setup over an air-water interface. Polarised light is completely transmitted if at the Brewster angle, but reflected in the presence of a layer of material on the surface. The Brewster angle for the air-water interface is $53^{\circ}$.

When a monolayer is introduced to the surface of the water, the difference in refractive index will cause a measurable change in the reflectivity. This reflected light can then be used to create a high contrast optical image of the lateral morphology of the deposited layer. Such a monolayer thickness is approximately $0.5 \%$ the wavelength of visible light which makes the relative effect of reflected light 
negligible and under normal conditions the film is invisible. By using polarised light and the Brewster angle, the introduction of a monolayer results in a visibly determinable film against a dark background which corresponds to the zero reflection characteristics of water. The Brewster angle microscope used in this work was the KSV NIMA microBAM, and is illustrated in fig 3.7. In this system, a $659 \mathrm{~nm},>20$ $\mathrm{mW}$ laser diode is directed towards the water surface at an angle of $53^{\circ}$ whilst the microscope is positioned approximately $1 \mathrm{~cm}$ above the water surface. Prior to measurement of the surface in the LB trough, an angled piece of black glass is placed directly under the surface of the water. This is to ensure the transmitted light ( $>99 \%$ of total energy) is largely absorbed or reflected at a large angle, away from the camera that visualises the surface, in order to prevent saturation of the image. The BAM can be used to study the phase behaviour of the deposited films, the influence of the subphase on the monolayer structures, and the homogeneity of the LB films, making it a very useful non-contact technique prior to deposition onto a solid substrate.

So far, the techniques to probe the characteristics of the formed film at the air-water interface have been outlined which can give some insight into the morphology of the film. Once deposited onto a substrate, the transferred film is open to a host of other sensitive characterisation techniques, which will be discussed in the following sections.

\subsubsection{Scanning Probe Microscopies (SPM)}

In this work, different SPM techniques allowed for the inspection of the nanoscale topographical, mechanical and conductive properties of the deposited thin films. It provides great complementarity with the other techniques that are capable of probing the surface on larger scales. All the scanning probe microscopy techniques employed in this work are derivatives of the atomic force microscope (AFM). Thus, the working principle of an AFM will first be described before summarising the differences of the other imaging modes. An AFM operates by measuring the force between a tip and the specimen under inspection, typically the tip is a 3-6 $\mu \mathrm{m}$ tall pyramid that reaches a diameter of $15-40 \mathrm{~nm}$ at its end. This results in the lateral resolution of the image being limited to approximately 
$30 \mathrm{~nm}$, whereas the vertical resolution can be as high as $0.1 \mathrm{~nm}$. For all measurements in the following chapters, a Bruker Icon AFM was used where the specific details are given in the chapters. The scanning probe microscopies used here allowed insight into the morphology, composition, height and lateral sizes of the films and its constituents deposited via the Langmuir-Blodgett method.

To acquire an image, an AFM measures the vertical and lateral deflections of a cantilever that is attached to the tip by using the optical lever effect. These deflections result in a laser that is incident on the cantilever being reflected to different regions of a 4 quadrant photodetector, the position of the laser on the detector then corresponds to the angular deflection of the cantilever. This concept is illustrated in fig 3.8. The sample is then attached to a piezoelectric slab that is used to move the sample in the $\mathrm{x}$ and $\mathrm{y}$ directions in order to scan across the surface.

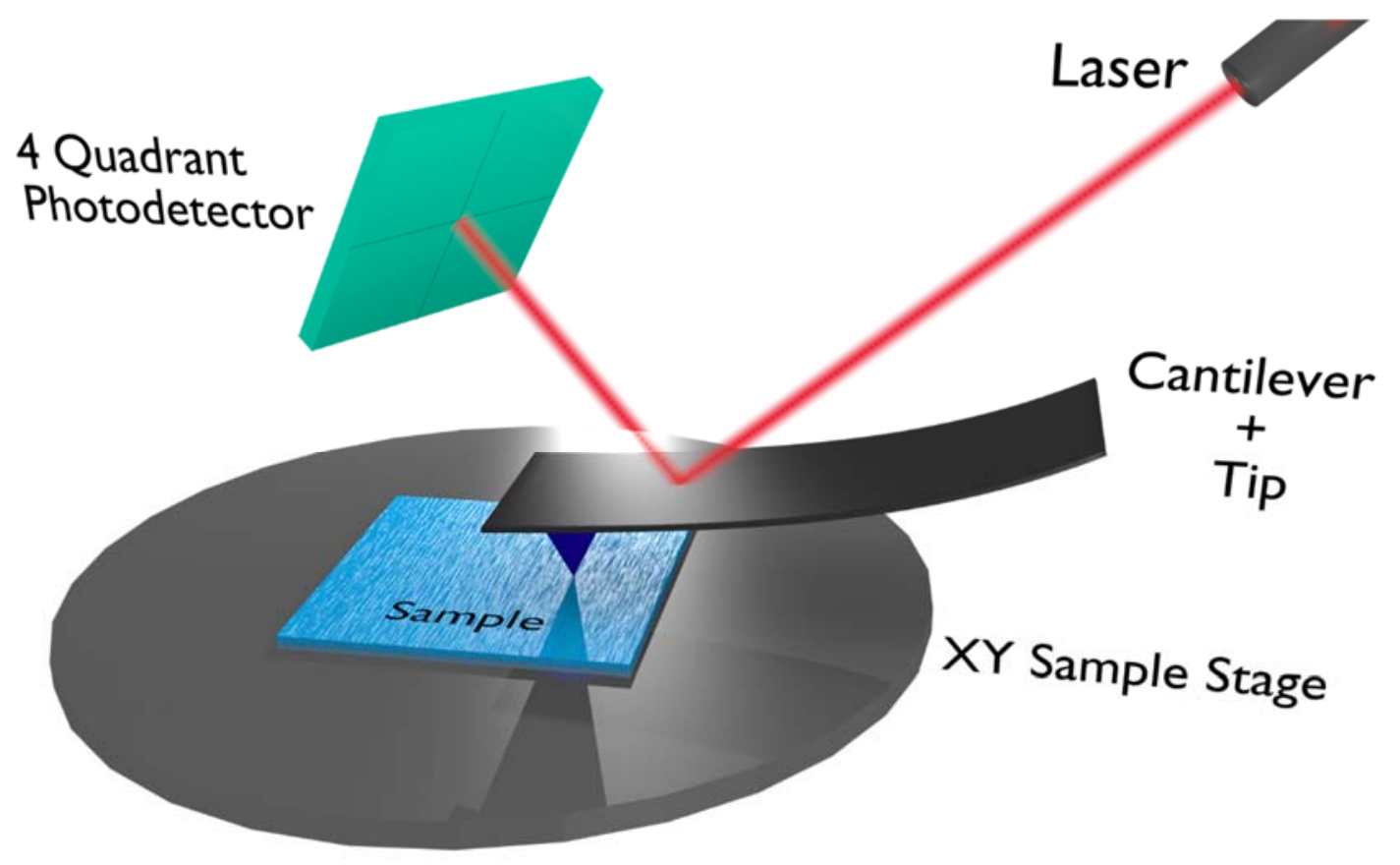

Figure 3.8: Schematic of the working principle of an atomic force microscope. As the tip is scanned across the surface, the height fluctuations causes the cantilever to twist and deflect a laser aimed at a 4 quadrant photodetector. Allowing the topography to be mapped.

There was two modes of imaging used in this work, namely contact and tapping mode. Contact mode operates through the tip being in direct contact with the sample surface. As the piezoelectric slab moves the sample underneath the probe, the photodetector signal is sent through feedback 
electronics that controls the z-height of the slab in order to keep the force on the cantilever constant; corresponding to a fixed tip-sample distance. The change in z-height is then measured across the sample surface to give the topography of the sample. An alternative scanning method is tapping mode. Here, instead of being in constant contact, the cantilever is oscillated at a fixed frequency such that the tip is only in contact with the surface for short periods of time. The height profile is then inferred from the variation of the amplitude of oscillation, which changes as the sample moves towards or away from the tip.

The parameters had to be carefully optimised in the AFM measurements of the nanoparticles in chapter 6, necessitating the use of tapping mode. If contact mode was used, the results that were achieved showed 'smeared out' nanoparticles due to the large lateral force applied, capable of dragging the nanoparticles across the surface. Even in tapping mode, the parameters had to be adjusted and optimised such that an extremely small amplitude and scan rate was applied to the sample. This facilitated the imaging of nanoparticles, although the resolution was reduced compared to the AFM's capabilities, due to some residual lateral movement of the nanoparticles. It is thought that the nanoparticles were not particularly well adhered and could be moved around due to their spherical shape, resulting in a weak substrate-nanoparticle interaction.

The two other techniques employed here are ultrasonic force microscopy (UFM) and conductive atomic force microscopy (C-AFM). UFM operates via oscillating the sample vertically at a frequency higher than the resonance frequency of the cantilever ${ }^{16,17}$. Measuring the resultant deflections and torsional vibrations can give information about subsurface and elastic properties and is able to discern materials of different elasticity by controlling the direction of vibrational forces. Most impressively, it can highlight the adhesion of a film of material to the sample and gives contrasting signals if a material is well adhered or completely decoupled from a substrate. It is this measurement that is principally investigated in this work for the fabricated thin films made via the LB technique.

C-AFM simply measures the current flowing through the tip and the sample and requires the use of a 
conductive tip. In principle, this technique measures one of the variants in Ohm's law. In the measurements taken in chapter 6 , a fixed $D C$ bias, $V_{i n}$, is applied to the sample and the measured current is converted to a voltage, $\mathrm{V}_{\text {out }}$, through use of a preamplifier. Through knowledge of the external resistance, $R_{\text {ext }}$, in the circuit, the resistance of the sample can be determined through the relationship $V_{\text {out }} / R_{\text {ext }}=V_{\text {in }} / R_{\text {sample. }}$ It should be noted that these two modes have to be operated whilst the AFM is in contact mode, but all three images can be measured simultaneously.

\subsubsection{Scanning Electron Microscopy (SEM)}

A scanning electron microscope was employed in the work presented here to gain insight into the morphology, coverage densities and size distributions of the films deposited via the LB technique. It allowed simple determination of the three-dimensional topographical and morphological data on a number of length scales. Furthermore, due to an SEM's large depth of field, large areas of the deposited films could be inspected simultaneously, making it an invaluable addition to characterise macroscopic areas with high resolution which the other techniques could not. By simple adjustments of the electromagnetic lenses within the system, the inspection of the films on a number of different length scales could be achieved efficiently; allowing examination from millimetres to nanometres. The SEM images presented in the following chapters were taken with a JEOL JSM-7800F system with acceleration voltages of up to $15 \mathrm{kV}^{18}$, and if it is not specified within the text, the acceleration voltage that was used was $5 \mathrm{kV}$, whilst the electron beam current was $20 \mathrm{pA}$ in all cases.

The main components of an SEM can be seen in fig 3.9. In the JEOL system, the electron beam is generated by a field-emission electron gun which extracts electrons using a large electric field generated by the potential difference between the gun and the anode. The gun is constructed of crystalline tungsten which is fabricated such that the tip is approximately $100 \mathrm{~nm}$, and the electrons are withdrawn from the very end of this tip. The beam is then passed through a series of lenses, known as electromagnetic lenses. These are essentially large coils of wire than can generate a magnetic field such that the electrons traverse a helical trajectory and come to a focal point once it exits the lens. 
Following this, the beam is sent through scanning coils, which use a magnetic field to direct the beam to the point of interest on the sample. These coils rapidly raster the beam over the sample area to produce an image. A number of apertures (not shown in fig 3.9) are used to reduce the beam diameter and increase the resolution. Moreover, the entire system must be placed under vacuum to prevent the electron beam scattering with air molecules.

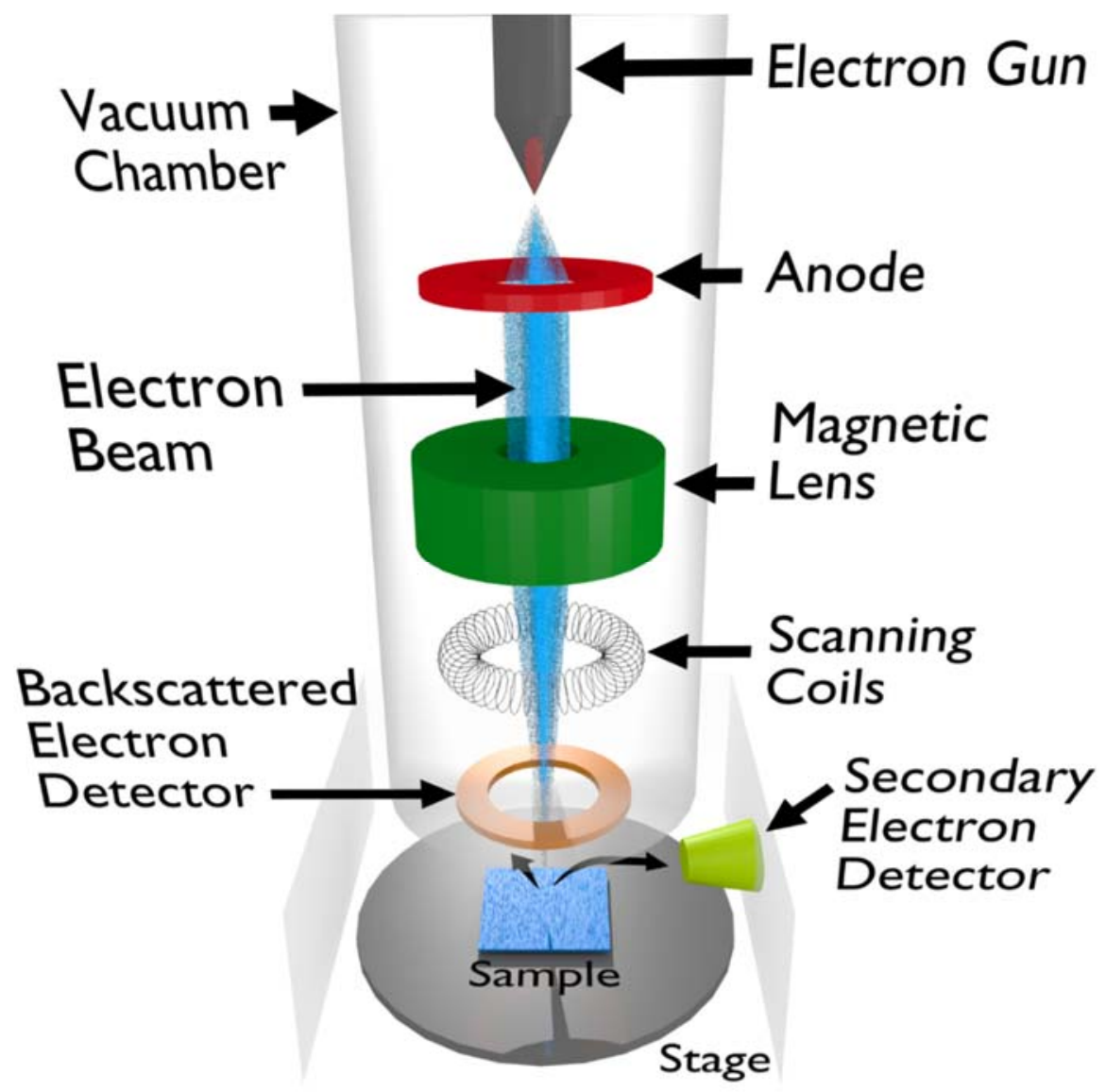

Figure 3.9: Main components of a scanning electron microscope. An electron beam is directed through a series of lenses and apertures so that it strikes a sample with a nanometre spot size. Scattered electrons are then detected to give structural and chemical composition of the sample.

Once the electrons strike the sample, two main types of signal can be detected to analyse the sample. Secondary electrons (SE) are generated when the electron beam dislodges electrons from the sample surface (depths of a few $\mathrm{nm}$ ) with a low-energy level. These electrons are then extracted via a detector that accelerates the electrons towards it using a Faraday cup. The electrons then interact with a scintillator connected to a photomultiplier tube. The amplified signal is re-converted to an electronic 
signal which then displays the image. The backscattered electrons are generated deeper within the sample and are reflected with approximately the same energy as the beam and detected via the same process as the SE. The secondary electrons give topographical data whereas the backscattered electrons give chemical composition. As no chemical composition was explored, all images presented here were taken with the secondary electron detector. The secondary electron images record the topographical and morphological data, and were therefore used for large scale characterisation of the deposited thin films of $\mathrm{MoS}_{2}$, nanoparticles and graphene in chapters 5 and 6 .

\subsubsection{Photoluminescence, Raman and Fluorescence Spectroscopies}

This section briefly discusses the methods used to study the interaction of the created films in chapters 5 and 6 with light. Using these methods allowed the investigation of quenching mechanisms of the $\mathrm{MoS}_{2}$ and the nanoparticles through measuring samples fabricated in different methods. Furthermore, it gave some complimentary insight into the thickness of the films of $\mathrm{MoS}_{2}$, where the Raman spectra is a good indicator of layer thickness. The micro-photoluminescence data was taken with a home built system shown in fig 3.10. Here, a continuous wave Nd:YAG frequency doubled 532 $\mathrm{nm}$ laser is directed along an optical path which controls the incident power of the laser on the sample (details shown in the figure). The laser is focussed onto the sample through a 50x, 0.65 NA objective lens such that the spot size is $\sim 1 \mu \mathrm{m}^{2}$. The emitted light is then collected with the same objective and analysed using an Andor Shamrock 500 spectrometer connected to a Newton silicon EMCCD that is Peltier cooled to $-85^{\circ} \mathrm{C}^{19}$. The photoluminescence measurements on the $\mathrm{MoS}_{2}$ in chapter 5 was carried out using this setup.

For the fluorescence measurements on the nanoparticles studied in chapter 6, an Agilent Cary Eclipse spectrophotometer was used ${ }^{20}$. As opposed to the laser used above, a Xenon lamp is used to excite the nanoparticle samples at $300 \mathrm{~nm}$, this was neccesary as the excitation wavelength needs to be far enough away from the band edge so that the fluorescence signal can be isolated. Furthermore, the light is incident over a large area of the sample such that the fluorescent signal collected is from the 
entire film. Also, to measure a spectrum, a photomultiplier tube is moved stepwise to collect the signal corresponding to each wavelength emitted from the sample.

The Raman data acquired in chapter 5 is taken using a Renishaw inVia Raman microscope ${ }^{21}$. The measurements were taken using the same $532 \mathrm{~nm}$ excitation laser as in fig 3.10. Raman spectroscopy allows the understanding of the materials vibrational energy levels through the interaction of the excitation laser with phonons in the material system. The main difference from the photoluminescence setup is that the filtering of the laser light must be extremely efficient, as the low energy phonons result in emission of light that is very close to the laser wavelength. In this work, Raman spectroscopy is used for identification of the thin film composition in chapter 5.

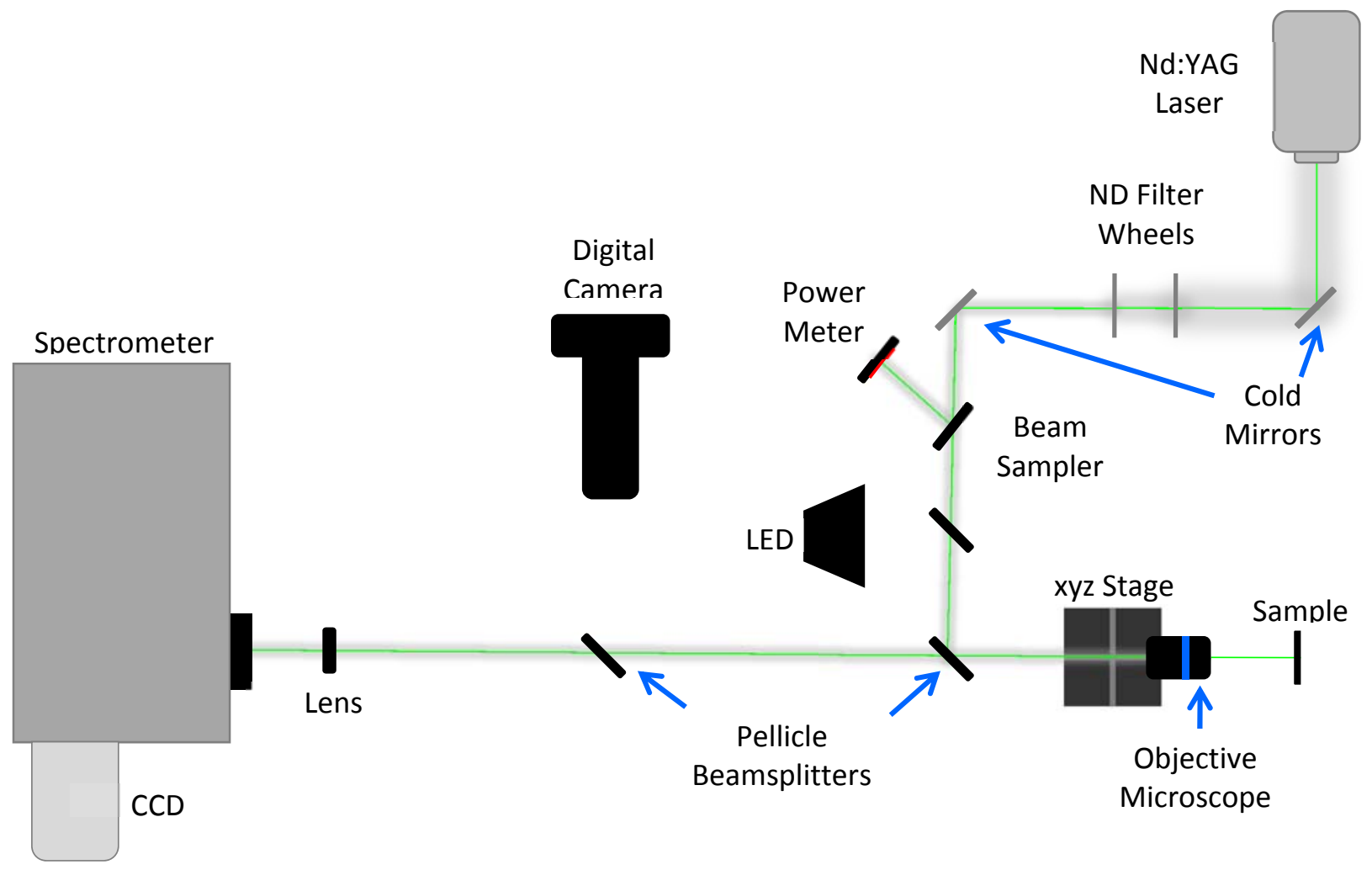

Figure 3.10: Micro-photoluminescence experimental setup. A $532 \mathrm{~nm}$ laser is directed via mirrors and beam splitters to impinge on a sample surface, the emitted light is collected through the same objective used for excitation and sent to a spectrometer and CCD. An LED is also used to optically image the sample. 


\section{Bibliography}

${ }^{1}$ M. A. M. Zawawi et al., "Fabrication of Sub-micrometer InGaAs/AlAs Resonant Tunneling Diode Using a Trilayer Soft Reflow Technique with Excellent Scalability", IEEE Transactions on Electron Devices, 61, pp. 23382342 (2014).

${ }^{2}$ www.wentworthlabs.com

${ }^{3}$ www.tek.com

${ }^{4}$ I. Langmuir, "The Constitution and Fundamental Properties of Solids and Liquids. II Liquids.", J. Am. Chem. Soc., 39, 1848 (1917).

${ }^{5}$ K. B. Blodgett, "Films Built by Depositing Successive Monomolecular Layers on a Solid Surface", J. Am. Chem. Soc., 57, 6 (1935).

${ }^{6}$ www.biolinscientific.com

${ }^{7}$ E. Sheppard \& N. Tcheurekdijian, "Monolayer Studies III. The Spreading of Polystyrene Latexes at Water/air Interface”, Colloid Polym. Sci., 225, 2 (1968).

${ }^{8}$ D. A. Walters, "A Stable Transitional Flow Pattern in the Surface Tension Driven Spreading of Ethanol-Water Solutions", Langmuir, 6, 5 (1990).

${ }^{9}$ L. J. Cote et al., "Langmuir-Blodgett Assembly of Graphite Oxide Single Layers", J. Am. Chem. Soc., 131, 3 (2009).

${ }^{10}$ J. Zeleny, "Instability of Electrified Liquid Surfaces", Phys. Rev., 10, 1 (1917).

${ }^{11}$ M. Cloupeau \& B. Prunet-Foch, "Electrohydrodynamic Spraying Functioning Modes: A Critical Review", J. Aerosol Sci., 25, 1025 (1994).

12 G. Taylor, "Disintegration of Water Drops in an Electrical Field", Proc. R. Soc. London A, 280, 383 (1964).

${ }^{13} \mathrm{H}$. Nie et al., "High-yield Spreading of Water-miscible Solvents on Water for Langmuir-Blodgett Assembly", J. Am. Chem. Soc., 137, pp. 10683-10688 (2015).

${ }^{14}$ C. N. Ryan et al., "The Flow Rate Sensitivity to Voltage Across Four Electrospray Modes", Appl. Phys. Lett., 104, 084101 (2014).

${ }^{15}$ D. Brewster, "On the Laws Which Regulate the Polarisation of Light by Reflection from Transparent Bodies", Phil. Trans. R. Soc. London, 105, 125 (1815).

${ }^{16}$ K. Yamanaka et al., "Ultrasonic Force Microscopy for Nanometer Resolution Subsurface Imaging”, Appl. Phys. Lett., 64, 2 (1994).

${ }^{17}$ K. Yamanaka, “Ultrasonic Force Microscopy”, MRS Bulletin, 21, 10 (1996).

${ }^{18}$ www.jeoluk.com

${ }^{19}$ www.andor.com

${ }^{20}$ www.agilent.com

${ }^{21}$ www.renishaw.com 


\section{Chapter 4}

\section{Unique Identification with Resonant Tunneling Diodes}

\subsection{Introduction}

Electronic devices have become extremely prominent in everyday life, and it is now commonplace for them to control critical tasks, such as managing financial transactions. Therefore, it is of key importance that these devices can be securely identified to prevent illegitimate parties mimicking themselves as genuine, and gaining access to sensitive information. The popular methods of identification currently in use rely on the end user providing some information about themselves, such as a fingerprint or a password, but these authentication methods are known to be extremely vulnerable. Identities can also be provided by systems that exploit physical disorder, but there is a growing need for the security to be as robust as physically possible. To this end, the use of quantum nanostructures that derive their identities from the atomic arrangement of the system would provide the ideal solution; made possible due to the sensitivity of quantum confinement on the morphology of the structure. Employing such a system would maximise the difficulty of cloning, providing a major barrier for nefarious parties, whilst containing the largest secure bit density possible due to the extremity in the device size. This chapter explores this concept by making use of an electronic device that exploits a simple quantum effect, tunneling. These devices, known as resonant tunneling diodes (RTDs), can provide an uncomplicated measurement of identity, corresponding to the straightforward measurement of the macroscopic current that passes through the device. This work represents the first step towards the realisation of a unique fingerprint based on the atomic-structure of a material. 


\subsection{Uniqueness in Semiconductor Nanostructures}

\subsubsection{A Sensitivity to Quantum Confinement}

Quantum confinement is extremely sensitive to the local compositional, structural and strain disorder of a semiconductor system ${ }^{1}$. This results in an array of apparently 'identical' devices displaying different properties at a device-to-device level. The fabrication of such devices through techniques such as molecular beam epitaxy (MBE) or metalorganic vapour phase epitaxy (MOVPE) has skyrocketed since their development in the $60 \mathrm{~s}$ and $70 \mathrm{~s}$, and these machines are now commonplace worldwide for building and designing a whole range of semiconductor structures, many of which exhibit quantum confinement.

Although the above fabrication methods have been finely tuned to mitigate the production of defects and other imperfections in the local structure, they cannot be eradicated completely. Such defects in these devices are unwanted, and there has been lots of effort in producing structures with identical characteristics ${ }^{2.3,4}$. The atomic layers within these devices are crucial for the underlying properties of the system, as the energy levels within such a device are sensitive to these layers ${ }^{5,6}$. For example, by using the particle in a box model, the effect of changing the well width can be shown. Here, the effective mass for GaAs at room temperature $\left(0.067 \mathrm{~m}_{0}\right)$ is used, whilst assuming a well width of $5 \mathrm{~nm}$. The effect of increasing the width of the well by $1,0.5,0.1$ and 0.01 monolayers results in a red-shift in the electrons energy level of $43.4,23.5,5.0$ and $0.5 \mathrm{meV}$, respectively. The inclusion of a fractional monolayer indicates that 50,10 or $1 \%$ of the quantum well's area has a height increase of 1 monolayer. Amazingly, the effect of increasing the well width by 1 monolayer in just $1 \%(0.01)$ of the device results in a 0.5 meV change; a change that can be measured experimentally ${ }^{7,8}$. During resonant tunneling, the electron's sample all these areas and take different paths through the device, resulting in a unique IV spectrum. As these layers contain millions of atoms, the probability of creating a unique device with each fabrication is extremely high, exemplified in fig 4.1. In this work, it is suggested to repurpose these imperfect devices that are seen by many as faulty, for a novel application in information security; namely the creation of the first UNO/PUF-type system that utilises atomic uniqueness. 


\subsubsection{Imperfections and Defects in Nanostructures}

A semiconductor is a periodic crystal structure that relies heavily on its underlying symmetry for the existence of their fascinating properties. Defects such as missing atoms, impurity atoms, lattice disorder and aperiodicity all play a role in perturbing this symmetry, which results in a profound effect on the physics defining its behaviour. Examples of this symmetry breaking include nitrogen vacancy centres in diamond ${ }^{9}$, donors and acceptors in III-V semiconductors ${ }^{10}$ and well width fluctuations in GaAs/AlGaAs resonant tunneling diodes (RTDs) ${ }^{11}$. Incredibly, the effect of changing just a single atom within tens of thousands can change the properties of the system due to the same symmetry breaking $^{12}$. As the presence of defects alter the electrical and optical properties of the system, the vast range of possible defects have received ample amounts of experimental and theoretical study, as it is an important topic for device fabrication. As an example, intrinsic defects such as vacancies and selfinterstitials can regulate some of the basic properties of a semiconductor, such as dopant diffusion.

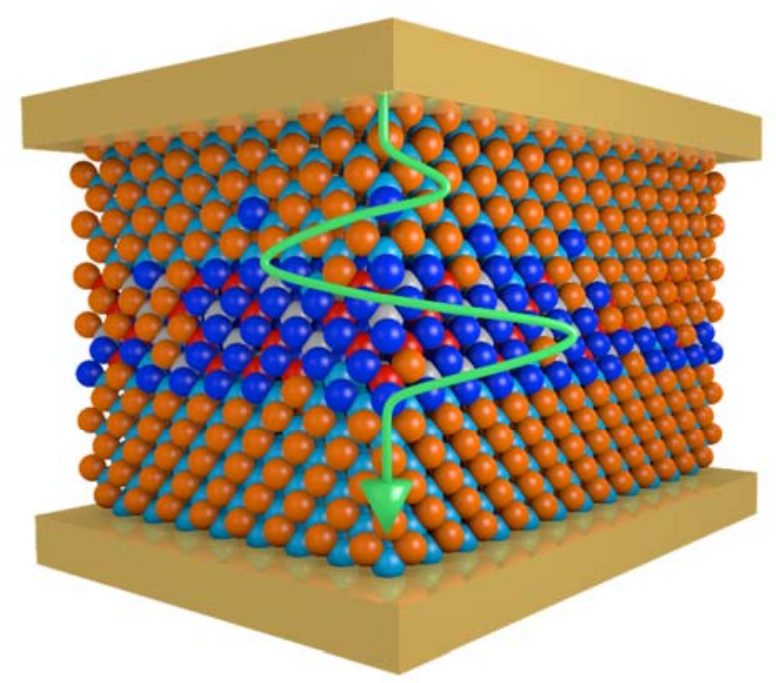

Figure 4.1: Randomness in semiconductor nanostructures show structural and compositional changes on a local device scale and can be used for unique identification.

Perhaps most poignant is the defects that arise at semiconductor interfaces; the adjoining of semiconductor crystals of different band alignments. These heterostructures have given rise to a number of devices with a variety of applications, from solar cells to LEDs. However, the defects that arise at the crystal interfaces are imaginably enhanced, and only worsen as the crystals being employed are taken to ternary and quaternary compositions ${ }^{13}$. Much could be said about the 
extensive range of studies on defects and imperfections in semiconductors systems but it is not in the scope of this work, and the reader is referred to comprehensive works on the topic ${ }^{14}$.

\subsubsection{Quantum Dots as an Example System}

The effects of such device variation can be conveniently demonstrated using the optical spectra of quantum dots grown via MBE, which provides a simple probe into the modified properties of defected structures. The production of quantum dots is conventionally completed through the StranskiKrastanov mode of growth. Here, layers of material with significantly different lattice constants are grown epitaxially which leads to a large build-up of strain within the semiconductor system. To alleviate some of this strain, the semiconductor changes its morphology to lie in its lowest energy state by separating into distinct islands of material; these islands are known as quantum dots. This relief of strain results in quantum dots that display many imperfections, as elucidated by crosssectional STM measurements of a typical quantum dot system ${ }^{15}$. The height, width and depth of these islands are commonly tens of $\mathrm{nm}$ in size, and as such this system confines electrons and holes in all three spatial dimensions, where the emission energy is dictated by the ground state energy of the electron and hole within the dot. This ground state energy shows large variations due to the inhomogeneous distribution of shape, size and composition of dots within the ensemble. Hence, finding two dots with identical composition and structure is extremely unlikely, and similarly, finding two with identical emission spectra is equally unlikely ${ }^{16,17}$. This is a key reason that implementing quantum dots as light sources has been problematic. Example spectra of a number of different quantum dots from the same growth procedure can be seen in fig 4.2, where it is observed that the excitonic states that are measured vary in energy considerably. In the case of fig 4.2 , the optical spectra arise from GaSb quantum dots embedded in a GaAs matrix and were measured with the microphotoluminescence setup introduced in chapter 3 , at a power of $0.3 \mu \mathrm{W}$. The variation in optical spectra is a clear indication that such a semiconductor system could be used for identification. 


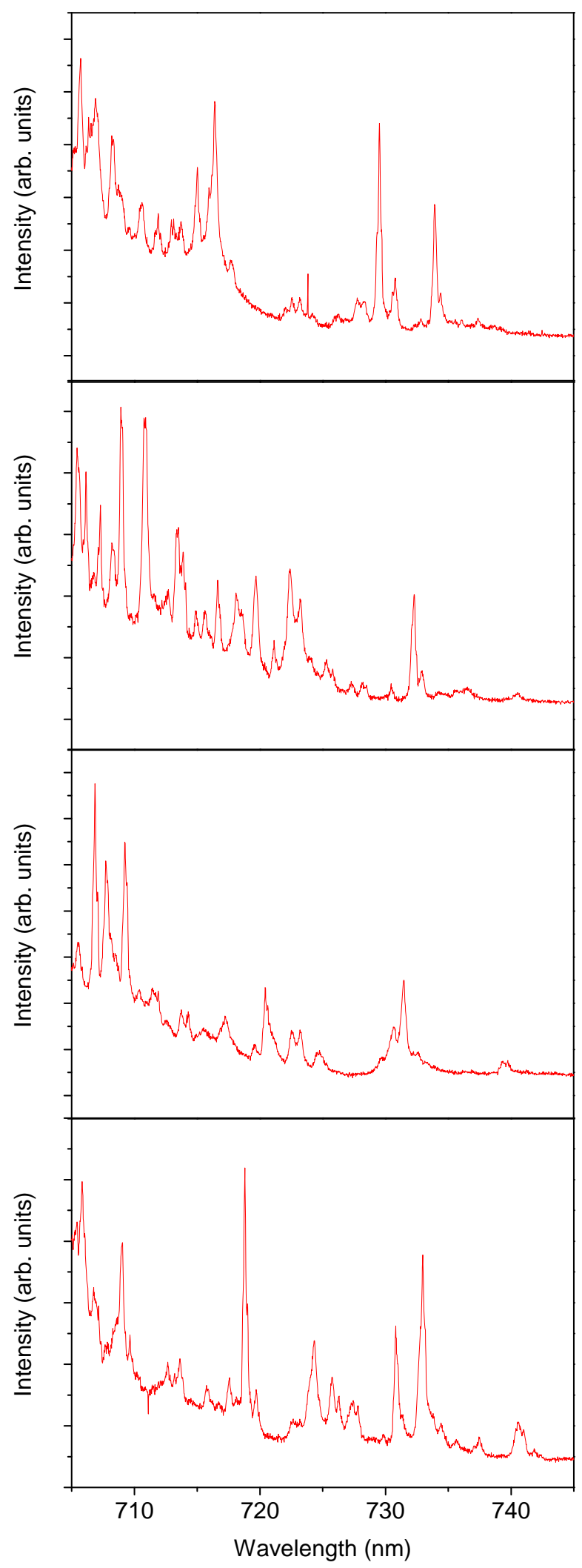

Figure 4.2: Emission spectra from different quantum dots within an ensemble. There are no consistent features from spectrum to spectrum indicating large fluctuations between the structure and compositions of the quantum dots; typical for quantum dot samples. Intensity scales are equal. 


\subsection{Electronic Unique Identification using Quantum Confinement}

\subsubsection{Resonant Tunneling Diodes}

The variations witnessed in the emission spectra of quantum dots derive their uniqueness from the quantum confined energy levels within the system. To use this concept for electronic identification, a simple device that can measure the quantum energy levels of the internal structure needs to be employed. To this end, the resonant tunneling diode (RTD) was chosen, as the room temperature current-voltage characteristic produced in these devices is directly associated to the confined energy level in the system. Specifically, RTDs make use of the concept of quantum mechanical tunneling, which facilitates the passing of electrons through barriers with widths comparable to the electrons wavelength.

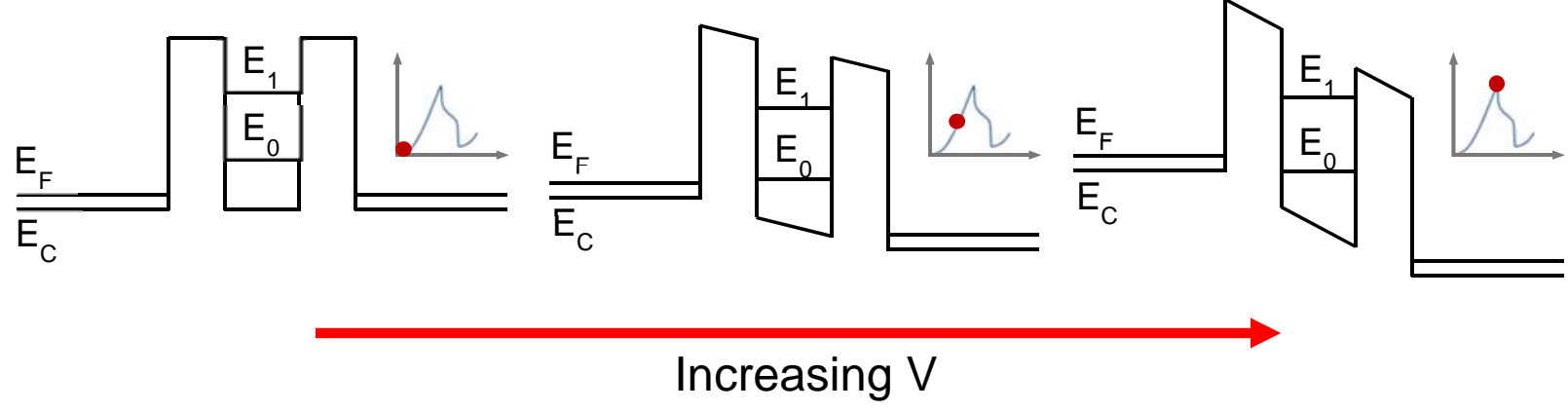

Figure 4.3: Band-diagram showing the process behind the resonant tunneling effect. As the voltage is increased, the confined energy level comes into resonance with the minima of the conduction band, leading to a maxima in current. The current diminishes with further bias due to reduction of this resonance and produces a characteristic negative differential resistance (NDR) region in the curve.

In classical physics, if an electron does not have high enough energy to overcome the barrier, it reflects at the interface. However, quantum mechanics provides a finite probability with which the electron can tunnel directly through the barrier; and this probability increases as the barrier is made thinner. Resonant tunneling diodes are based on a double potential barrier structure that sandwiches a quantum confined nanostructure, which contains a series of energy levels. Electrons in the Fermi Sea, the statistical distribution of carriers outside the barriers, can tunnel through the entire structure when one of the confined energy states is equal to the energy of the electrons outside the barriers. 
To understand the current-voltage spectra, the reader is referred to fig 4.3. When there is no bias across the device, only a small fraction of electrons in the Fermi Sea have an energy equal to the bound energy state, so a negligible current is observed. As the bias begins to increase and the band structure undergoes a Stark shift, the confined energy level approaches the Fermi level, which allows a greater number of electrons to tunnel through the device. At a specific voltage, the confined energy level comes into resonance with the minimum of the conduction band, giving rise to a maximum in the current due to the large density of states at this level. As the bias is increased beyond this point, a negative slope known as the negative differential resistance (NDR) region is seen, due to less electrons being in resonance. At high bias voltages, the current rises rapidly due to thermionic emission over the barrier or due to the second resonant energy level ${ }^{18}$.

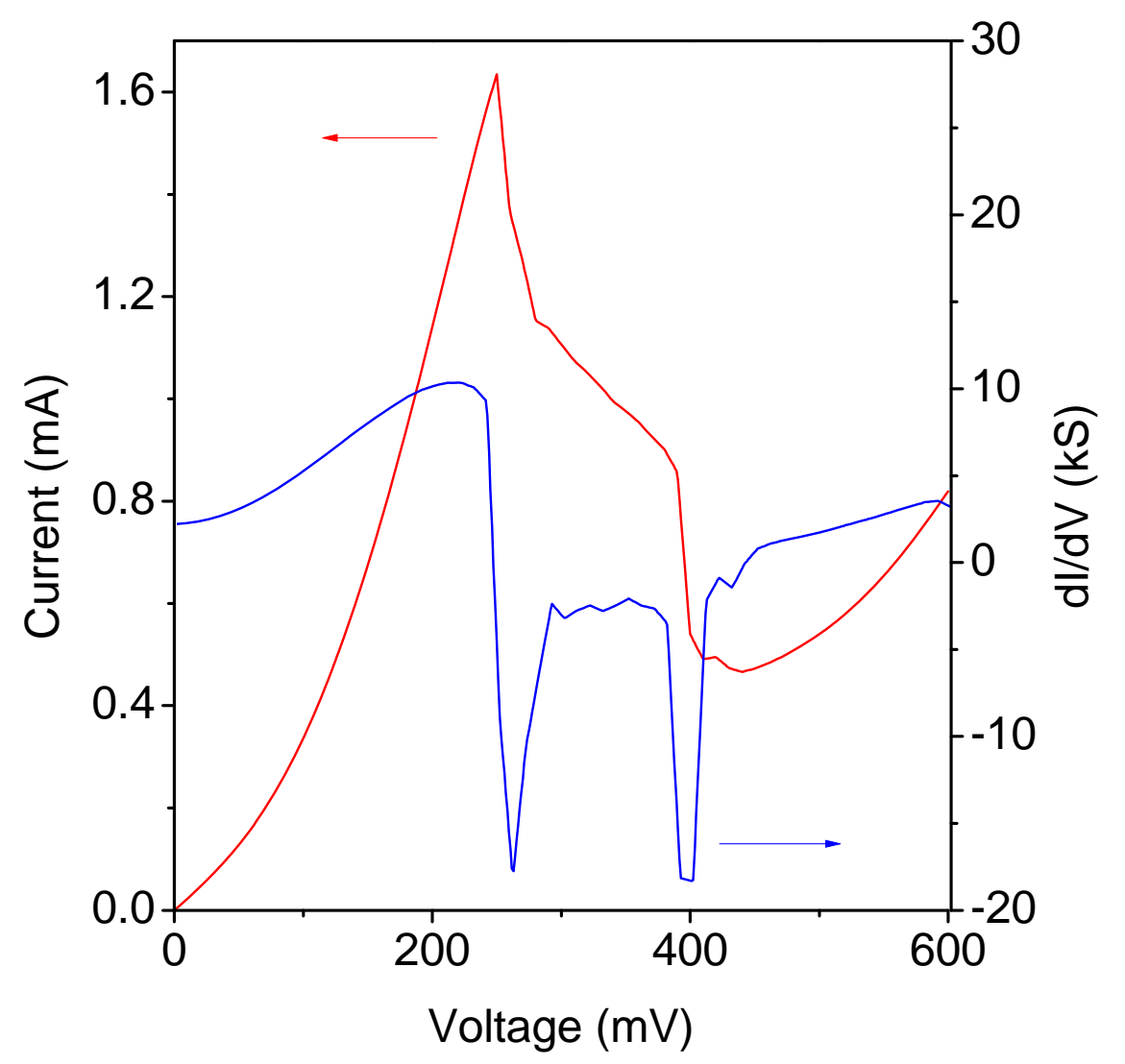

Figure 4.4: A typical current-voltage spectra from a resonant tunneling diode and its derivative. The peak in current is associated with the confined energy level within the RTD, and the plateau arises from the coupling to an 'emitter quantum well' due to band bending. The $d l / d V$ is often included to deconvolute the classical and quantum physics of the system. 
The resultant I-V spectra that results from increasing the bias whilst measuring the current of a typical RTD is shown in fig 4.4 (red trace). The $\mathrm{dl} / \mathrm{dV}$ spectra (blue trace) is often used to characterise an RTD as it provides an insight into the NDR region. The constantly increasing slope that is measured as the bias in increased does not produce any noticeable features in the $d \mathrm{l} / \mathrm{dV}$ spectrum. However, when there is a large change in the gradient of the I-V, such as at the peak, the $\mathrm{dl} / \mathrm{dV}$ exhibits a clear peak corresponding to this immediate gradient change. Thus, using the differential conductance allows a convenient method to deconvolute the classical and quantum physics of the system. The plateau-like region observed following the current peak was the source of much controversy for a long period of time, being attributed to either intrinsic charge stabilities within the device itself ${ }^{19}$, or external circuitinduced high-frequency oscillations in the NDR region ${ }^{20}$. However, it is now thought to arise from the coupling of the main quantum well, and the small 'emitter quantum well' that forms on the outside of the barrier due to the band bending caused by the application of a bias ${ }^{21}$.

(a)

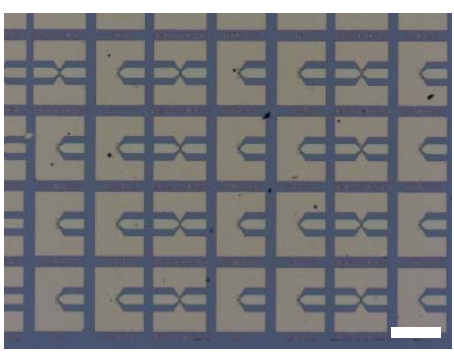

(b)

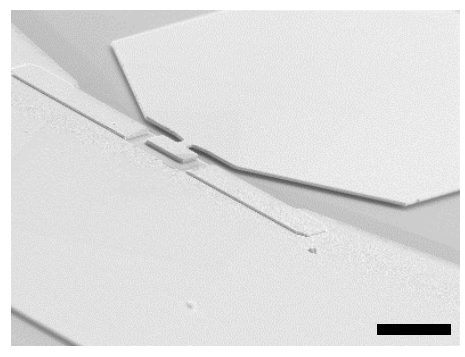

(c)

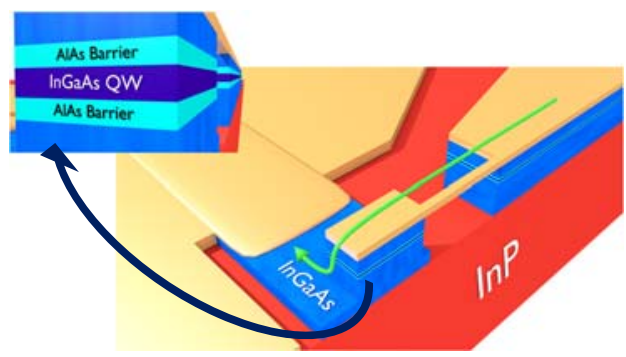

Figure 4.5: Resonant tunneling diodes used in this work, showing (a) section of the chip (scale bar 200 $\mu \mathrm{m})$, (b) SEM of an individual device (scale bar $10 \mu \mathrm{m}$ ) and (c) rendered counterpart of the device, highlighting the important features (an AlAs/InGaAs/AlAs double-barrier structure).

Although RTDs can contain an array of nanostructures, in this work a quantum well RTD was used, as this offers the 'minimum amount' of uniqueness, with only one dimension of confinement. However, it allows the demonstration of a proof-of-principle whilst allowing room for improvement in the uniqueness of such devices. Fig 4.5a demonstrates the array of devices used in this work, showing the contact pads which are used for testing, fig $4.5 \mathrm{~b}$ shows an SEM image from a single device whereas fig $4.5 \mathrm{c}$ shows a rendered counterpart to highlight the important features. Specifically, this is an InGaAs 
quantum well surrounded by AlAs barriers ${ }^{22}$. The mesa containing the double-barrier structure is connected to the contact pad via an air-bridge of various sizes which is used to separate the RTD from the main contact whilst allowing the reduction of the device area.

The confined levels are sensitive to the width and potential height of the barriers and of the well, and as such on the atomic uniformity at the interfaces between these layers that produce the most defects. A number of works have been reported that show just how sensitive these layers are to the resulting measurement ${ }^{23,24,25,26,27}$. Specifically, typical tunneling devices can display a variation of up to $270 \%$ in their I-V characteristics when interfacial fluctuations on the order of 1 monolayer exist ${ }^{27}$. To improve this, considerable effort has been devoted to produce highly uniform I-V spectra from tunneling devices, with current state-of-the-art systems reaching a variation of $1 \%$ about a pre-defined mean $^{26}$. This value corresponds to only a 0.02 monolayer variation over an 8 " wafer. These impressive works were done with single-barrier systems employing only binary crystals in their core, thus relying on only two binary-binary interfaces. The results presented here rely on four binary-ternary interfaces which results in much larger fluctuations in the width and position of the confined energy level within the quantum well ${ }^{28}$.

\subsection{Testing RTD Characteristics}

\subsubsection{Stabilisation of RTDs}

As there was a large array of devices present on the chip, the probe station was employed for all measurements to allow them to be measured efficiently without requiring individual bonding. To ensure the reliable reproduction of results from measured RTDs, the integration and delay time (discussed in chapter 3) were varied. Moreover, the number of steps in the staircase sweep was varied to find the number of steps that led to a good resolution whilst decreasing noise in the I-Vs.

The integration time is related to the measurement speed (the number of power line cycles, NPLC), which can be varied by choosing a value between 0.01 and 10 for the measurement speed, which takes into account the operation frequency $(50 \mathrm{~Hz}$ in this case) to give an integration time between 
$0.01 / 50 \mathrm{~Hz}(0.2 \mathrm{~ms})$ and $10 / 50 \mathrm{~Hz}(200 \mathrm{~ms})$. The DC voltage and current are affected by power line induced noise which is periodic, however if the analog-to-digital converter integrates for a period of time equal to one cycle, then this signal noise can be cancelled out. In general, greater integration times result in reduced noise, but there is a trade-off between measurement time and accuracy. In this case, upon choosing a very long integration time of $200 \mathrm{~ms}$, it was found that this gave extremely unstable results. It is expected that this is due to overheating of the device due to the source being applied over very long periods of time (a typical 200 point measurement with 50 ms delay time would take $100 \mathrm{~s})$. Therefore, it was decided to investigate integration times that were much lower and on the order of a few milliseconds. Fig 4.6 shows the effect of changing the NPLC value and measuring the same device, 10 overlapping measurements were taken by using a set delay time of $50 \mathrm{~ms}$ and 200 steps in the measurement. It can be seen that changing the integration time has a small effect on the acquired result, which suggests that the measured current from the RTD is extremely stable. From the observed spectra however, values of $1.2 \mathrm{~ms}$ and $2.0 \mathrm{~ms}$ seemed to give the best results. Therefore, $1.2 \mathrm{~ms}$ was selected as the integration time (corresponding to an NPLC value of 0.06 ) to use for all following measurements, as this reduces the overall measurement time of the sweeps which in turn will reduce heating effects.

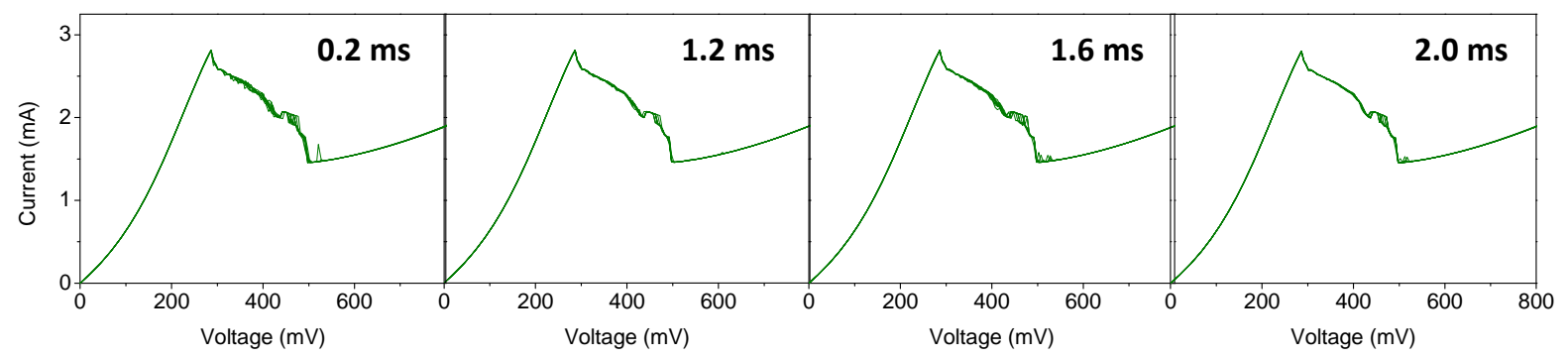

Figure 4.6: Results of 10 overlapping current-voltage spectra measured from a single device but taken with different NPLC values in the operation cycle, corresponding to integration times indicated in the different panels.

Using this measurement time, the variation caused by changing the delay time was studied. The delay time could be changed to between $0 \mathrm{~s}$ and a maximum of $9999 \mathrm{~s}$, but due to the oscillatory nature of RTDs, trial and error was used. It is known that for purely resistive loads, this value should be set to approximately zero, but RTDs have a small capacitive element (see section 3.2.3). By using the simple 
resistor-capacitor model for the RTD, the time constant can be calculated to estimate how long the delay should be. To provide an upper limit for the resistance, the minimum gradient was extracted from the I-V relationship in fig 4.6 (corresponding to the plateau region) and a maximum resistance of $\sim 50 \mathrm{k} \Omega$ was found. The capacitance can be estimated by using the formula for a parallel plate capacitor, $C=\varepsilon_{0} \varepsilon A / d$, where $A$ is the area of the device, $\varepsilon$ the dielectric constant of the material, and $d$ the distance between plates. To extract an estimate, the distance between plates is estimated as the distance between doped regions of the RTD mesa, $46.9 \mathrm{~nm}$. The dielectric constant of $\operatorname{In}_{0.53} \mathrm{Ga}_{0.47} \mathrm{As}, 13.9$, was used as this comprises $85 \%$ of this region. This results in a value of $1.05 \mathrm{fF}$, for a device area of $2 \times 2 \mu \mathrm{m}^{2}$. The resulting (maximum) time constant of these RTDs is therefore $0.53 \mathrm{~ns}$. For a circuit to stabilise (to $99 \%$ of set value), it is required that the delay time must be $5 R C$, using $\mathrm{V}=\mathrm{V}_{0}\left(1-\mathrm{e}^{\mathrm{t} / \mathrm{RC}}\right)$. Thus it was evident that the delay time is not required to be set much greater than zero, and trial and error of values about zero was performed to maintain a quick measurement protocol and avoid heating. 10 overlapping results of the same device with various delay times are displayed in fig 4.7, with the number of points here also being 200 . It was found that the optimum delay time was $80 \mathrm{~ms}$, this reduced the noise in the measurements most effectively and displayed the most accurate overlap between separate traces. To check these values are adequate across other devices, 10 other devices were tested and displayed similar reliability.

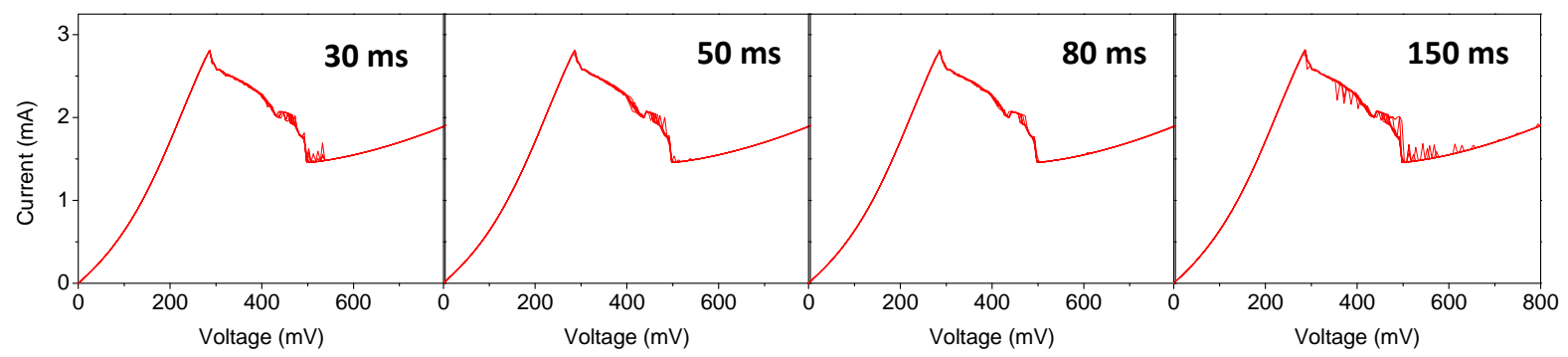

Figure 4.7: Results of 10 overlapping current-voltage spectra measured from a single device but taken with different delay times in the operation cycle, as labelled in the different panels.

Finally, a device was probed using these settings whilst changing the number of steps within the sweeps from 100-500. The results of 10 overlapping measurements are displayed in fig 4.8. It was observed that the overlapping current-voltage spectra got less consistent with an increasing number 
of points, where the increase in width of the overlapping traces is actually a result of their slight offset. In contrast to this general trend, the measurement acquired with 400 points displayed repeatable spectra whilst providing high resolution, so initially this number of points was employed for testing.

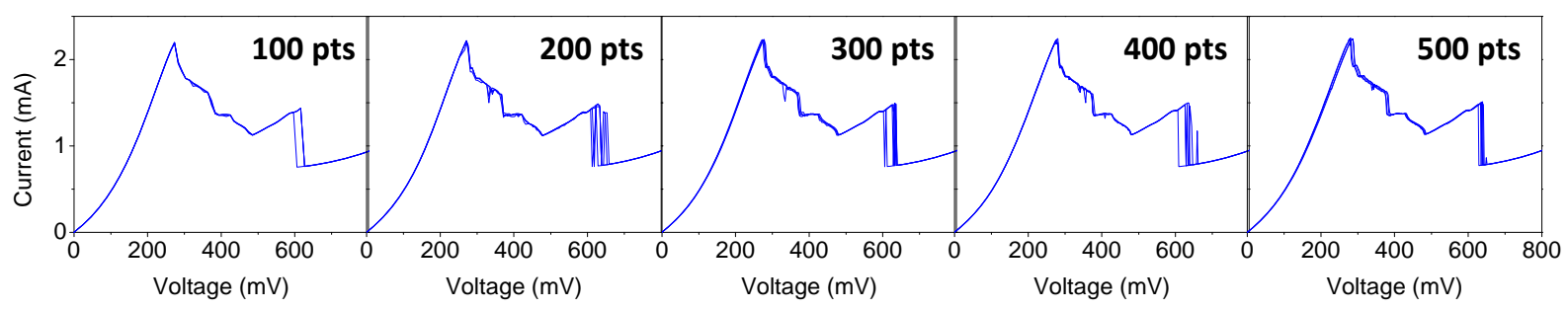

Figure 4.8: Results of 10 overlapping current-voltage spectra measured from a single device but taken with a different number of points in the sweep, as labelled in the figure.

However, upon measurement of other devices using 400 points and the previously ascribed values, the inconsistency of the measurements was repeatably seen, resulting in another value be chosen for the number. It can be seen in fig 4.8 that when the number of points is 100 , the traces overlap extremely well whilst removing some of the noisy spikes from the measurement. This is likely due to the averaging of the current readings as they are only being taken every $10 \mathrm{mV}$, but allows the peak value to be read consistently. Therefore in all measurements that follow, 100 points per spectra were used during each measurement.

\subsubsection{Size Selection of RTDs}

The resonant tunneling diodes that were available on the chip came in sizes that varied between $6 x$ $6 \mu \mathrm{m}^{2}$ and $2 \times 2 \mu \mathrm{m}^{2}$. It was of interest to find which size produced the most repeatable results as these would be the RTDs that were used for testing for use as unique identifiers. The data in fig 4.9 displays the data acquired from a typical device of these various sizes. The results in fig 4.9 show the large variations measured in the acquired spectra from the different sizes of RTD.

The enhancement in the tunneling current for different sizes is a consequence of varying the device geometry, where a device with a larger area allows a greater parallel classical current to flow due to the existence of more available electrons within the Fermi Sea. The reduction of the device area 
normally results in a significant decrease of the peak current density, due to the large series resistance increase associated with undercutting of the device ${ }^{29}$.

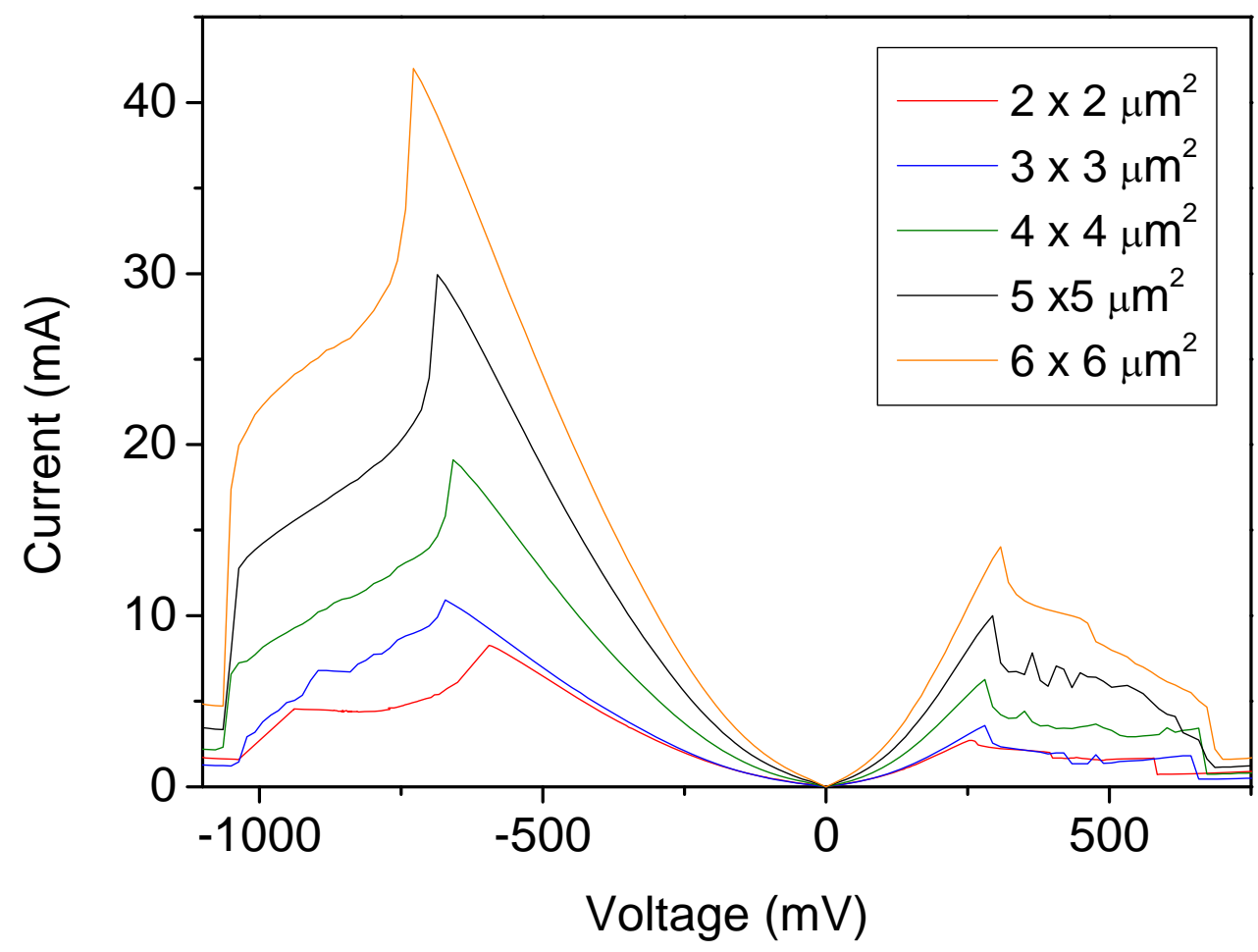

Figure 4.9: Modulus of current against voltage spectra from the different sizes of RTDs. The increase in current flowing through larger devices is due to the larger device area, with different geometries having similar current densities. The trend of decreasing voltage with decreasing device area is associated with the voltage drop across parasitic resistances in the circuit, as in ref [30]. $2 \times 2 \mu \mathrm{m}^{2}$ devices in forward bias were chosen from this selection due to their low I-V peak, which provided more robustness.

However, in the RTDs employed here, the peak current density shows excellent scalability, irrelevant of the size of the RTDs ${ }^{22}$. Considering that the area of these devices is increased by 9 times, the resulting shift is extremely low compared to other systems which show a similar shift for a 2 times increase in device $\operatorname{area}^{30}$.

For clarity, the modulus of current has been taken on the reverse bias side, and in reality this current is negative. The devices in this figure all comprised of a square mesa of the varying sizes connected by a $2 \mu \mathrm{m}$ air bridge to the contact region. It should be noted that although the RTD has a symmetric structure, the asymmetry of forward and reverse bias regions is due to the different doping profiles 
built into the collector and emitter regions, respectively. It is observed that the device which has the smallest current travelling through it is the $2 \times 2 \mu \mathrm{m}^{2}$ device operating in forward bias. This is crucial as the larger devices transmit a higher number of electrons and can result in inconsistent results, some of which are displayed in fig 4.10 .

(a)

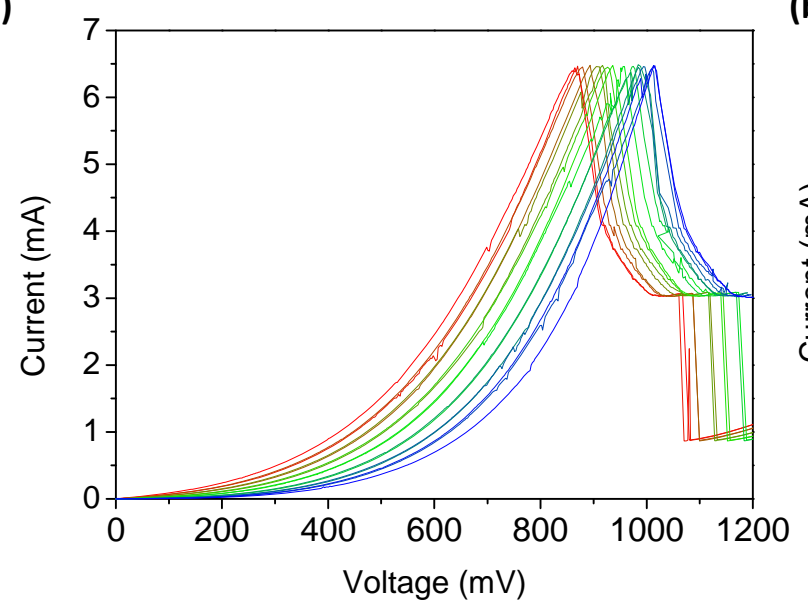

(b)

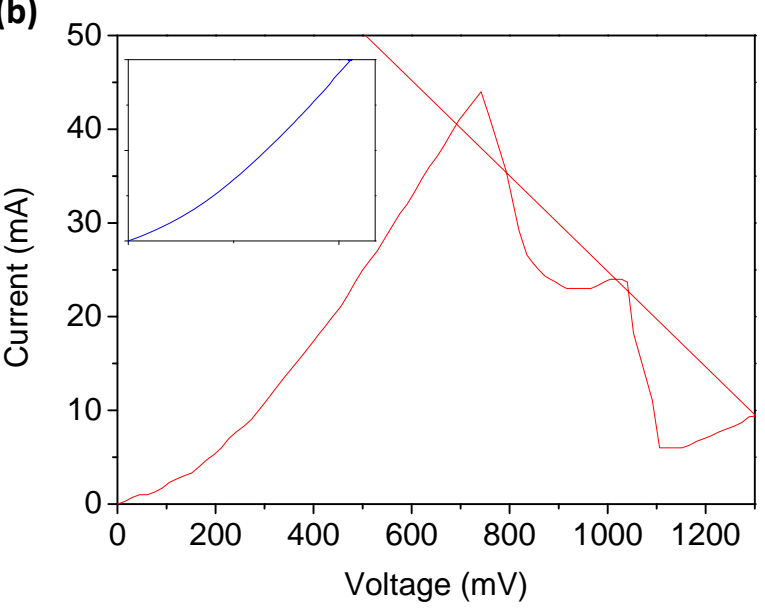

Figure 4.10: Various effects of unreliable results from using larger device showing (a) the peak of a device shifting due to heating and (b) the characteristic of a device failing through overcurrent. Inset: $\mathrm{I}-\mathrm{V}$ spectra following breakage, with same data scales as in (b).

For example, in fig 4.10a it is shown that a $4 \times 4 \mu \mathrm{m}^{2}$ device displayed a shift in the peak voltage which as mentioned earlier, is attributed to heating. Fig $4.10 \mathrm{~b}$ shows the signature of a typical device undergoing critical device failure, which completely destroys the nanostructure due to thermal degradation of the interfaces ${ }^{31}$. It was observed that this happens most often in large devices, $6 \times 6$ $\mu \mathrm{m}^{2}$ in this case, as the compliance of the source measurement unit must be set to a higher value; albeit just above the peak value. The inset displays the results obtained following failure of a device, which shows that the negative differential resistance region has been lost and indicates that the barrier-well region has essentially fused together to provide a parallel conductance channel. Finally, care must be taken to ensure that the probes are well placed onto the RTD's contact regions, as the addition of an external resistance due to a bad contact can lead to further instabilities in the RTD as shown in fig 4.11 . 
For all the measurements in the sections to follow, the optimised parameters described above were used, whilst establishing a systematic way of connecting the device to avoid the problems present in fig 4.11. This was done by placing the probes at approximately the same position on each contact pad, whilst avoiding movement once positioned on the contact pad which can promote removal of the gold contact layer. Furthermore, for simplicity of results, only the positive bias of the devices were used.

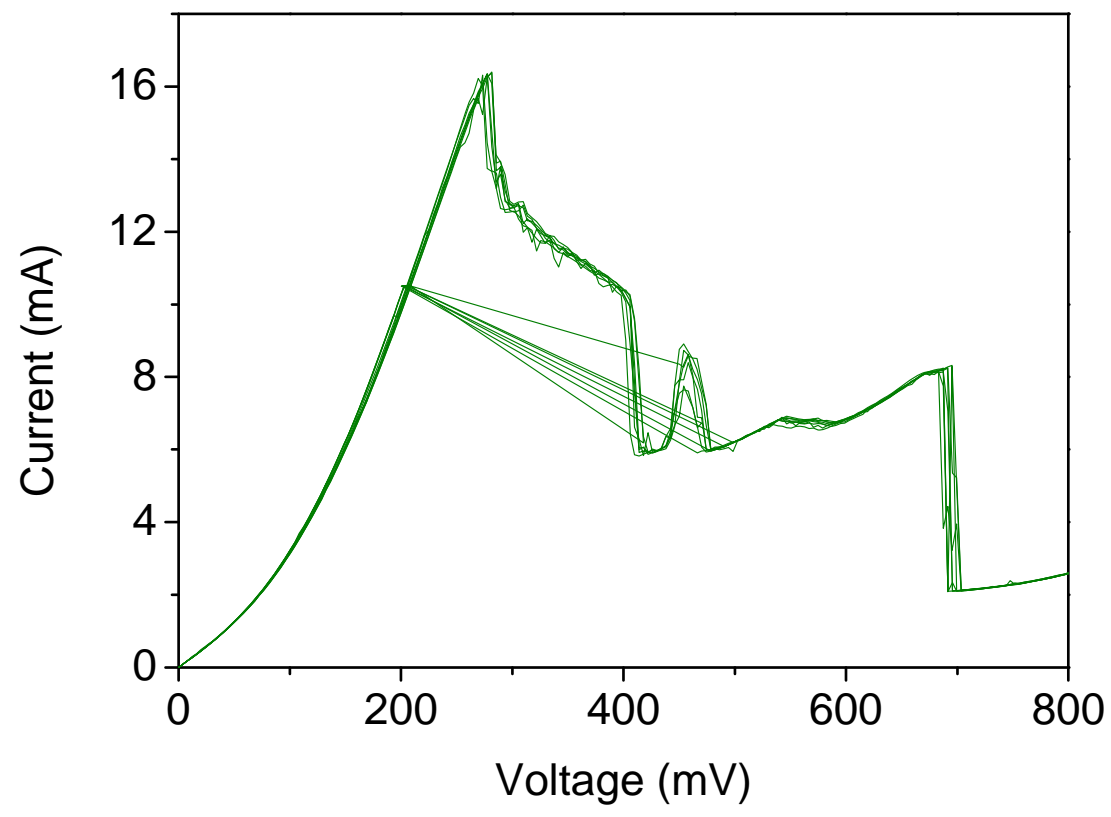

Figure 4.11: Oscillations present in the measurements of RTD's due to instabilities produced by external resistances present in the circuitry. Such resistances were mainly a result of creating a bad connection between the probes and the device, which consequently increases the contact resistance.

\subsection{Resonant Tunneling Diodes as Unique Identifiers}

\subsubsection{Testing the Uniqueness}

For unique identification of devices, it is clear that no two devices are allowed to display the same peak characteristics. Therefore, to justify using these quantum tunneling measurements as a reliable metric of uniqueness, 26 devices with identical fabrication steps were investigated and their results are plotted in fig 4.12. Specifically, these devices were $2 \times 2 \mu \mathrm{m}^{2}$ square mesas connected to a $3 \mu \mathrm{m}$ air bridge. The number of identical devices on the chip was limited to 26 , which prevented inspection of a larger number of devices. 
In fig 4.12a, it was observed that there was a $~ 50 \mathrm{mV}$ and $\sim 3 \mathrm{~mA}$ spread of the device's current-voltage peak. Upon closer inspection of the different spectra, it was evident that a number of traces displayed very close overlap (around $250 \mathrm{mV}$ and $2 \mathrm{~mA}$ ). It is likely that the average widths of the quantum well/barriers and the device area are approximately equal for these devices. These spectra were the results of averaging the acquisition of $100 \times 0->800 \mathrm{mV}->0$ sweeps of a device. On the other hand, the differentiated spectra plotted in fig $4.12 \mathrm{~b}$ showed that the peak produced from the gradient of the negative differential resistance region was much more consistent. Thus, for unique identification purposes, the peak position in the I-V spectra was used.

(a)

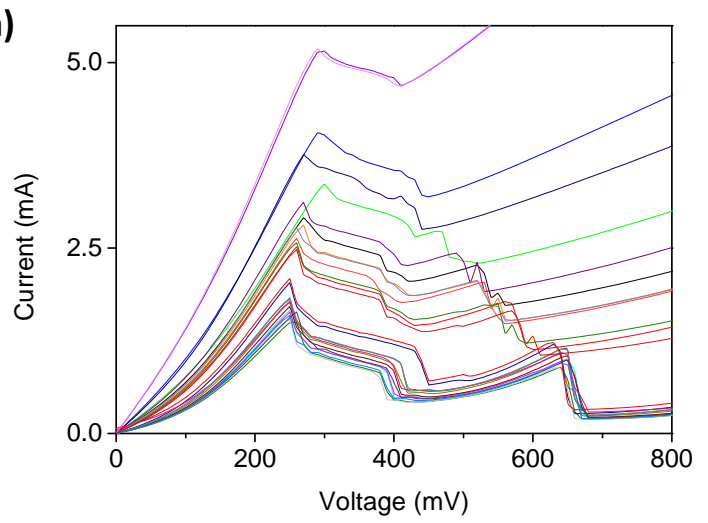

(b)

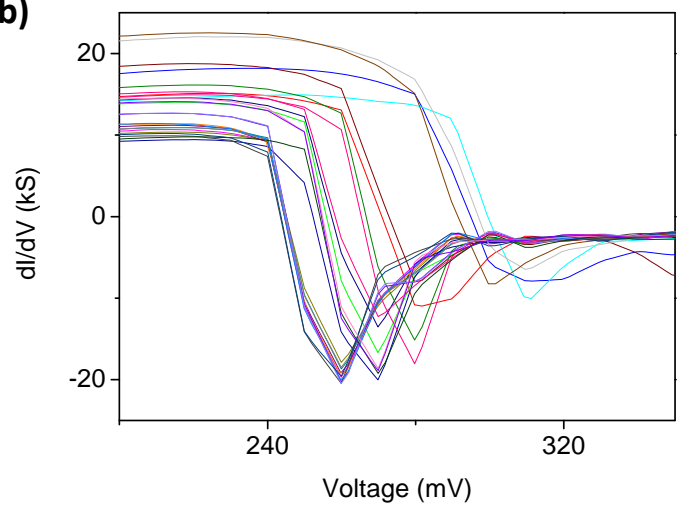

Figure 4.12: Results of testing 26 identical $2 \times 2 \mu \mathrm{m}^{2}$ devices, showing (a) I-V spectra and (b) dl/dV spectra. The I-V spectra showed a large variation in device characteristics although there seemed to be a group appearing at a specific I-V position, indicating all RTDs 'intended' peak position. Much greater consistency was observed in the $\mathrm{dl} / \mathrm{dV}$ spectra.

The signature from an RTD can be approximated as an exponential convoluted with a Gaussian ${ }^{32}$, where the Gaussian represents the tunneling current through the energy level, whilst the exponential arises from the normal diode current ${ }^{33}$. Thus, to further elucidate the variation between peak positions, Gaussian functions were systematically fitted to the peaks to extract the peak position and height. The resulting data is shown in fig 4.13 . Here, it can be seen that there is no collision in peak position, other than the area highlighted by the boxed region, which correspond to the close spectra in fig 4.12. However, this is an artefact of the symbol size. It was observed that there is a range in current and voltage values of $4 \mathrm{~mA}$ and $70 \mathrm{mV}$, respectively. This suggests that the peaks for other 
devices could fall (at least) anywhere within this range, although there is a clear positive correlation in the values, suggesting a strong Ohmic relationship. Thus, it is expected that high current-low voltage peaks or vice versa, will not be observed. Furthermore, the associated errors in voltage and current extracted from the spread in results are plotted but are too small to be visible in fig 4.13.

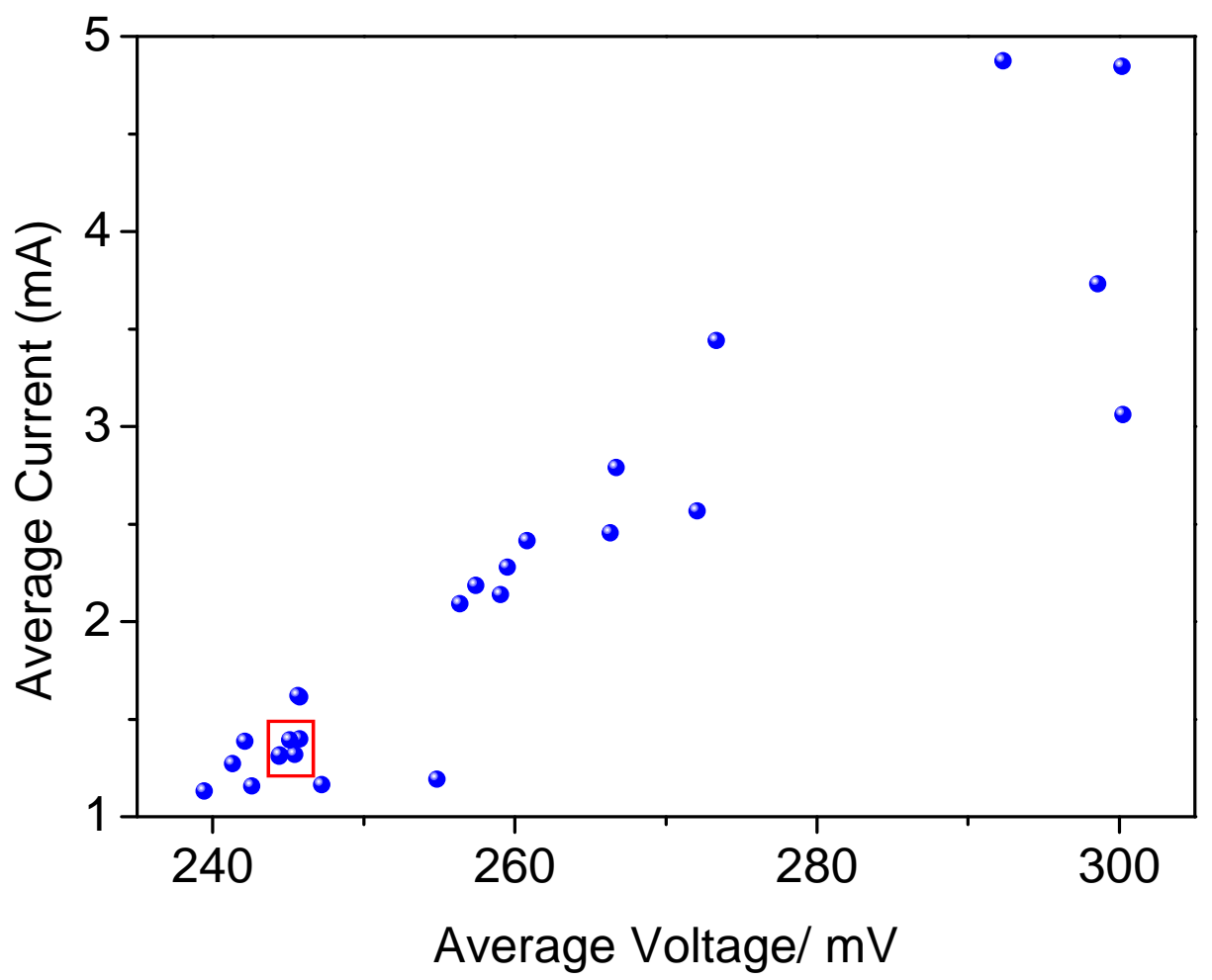

Figure 4.13: The average peak position of the 26 devices, calculated by averaging over 100 spectra that were measured from a given device. Positons have been extracted using Gaussian fittings, whose use is justified in the text.

Through closer inspection, it was observed that the devices within the red area were, too, unique. To highlight this, the data is re-plotted in fig 4.14. To estimate the confidence that the peak positions do not overlap, the 5 results obtained from the boxed region in fig 4.13 are plotted with their associated confidence ellipses about their average value. The ellipses were plotted by using the standard errors in voltage as the semi-major axis and the standard error in current as the semi-minor axis. Here, the 200 individual spectra of each device were analysed to find the peak voltage and current. These values were then used to find the average value of voltage and current for a given device, along with the standard deviation. This was converted to the standard error by using the number of values in the 
dataset. By using 1.96, 3.09 and 3.99 standard errors allowed ellipses of 95\%, 99.9\% and $99.997 \%$ precision confidence to be plotted. It was found that even using a $99.997 \%$ confidence ellipse resulted in no overlap between devices, although the exact position will be affected by the different resistances associated with each device and the heights and widths will be related to the interfacial roughness and exact device geometry.

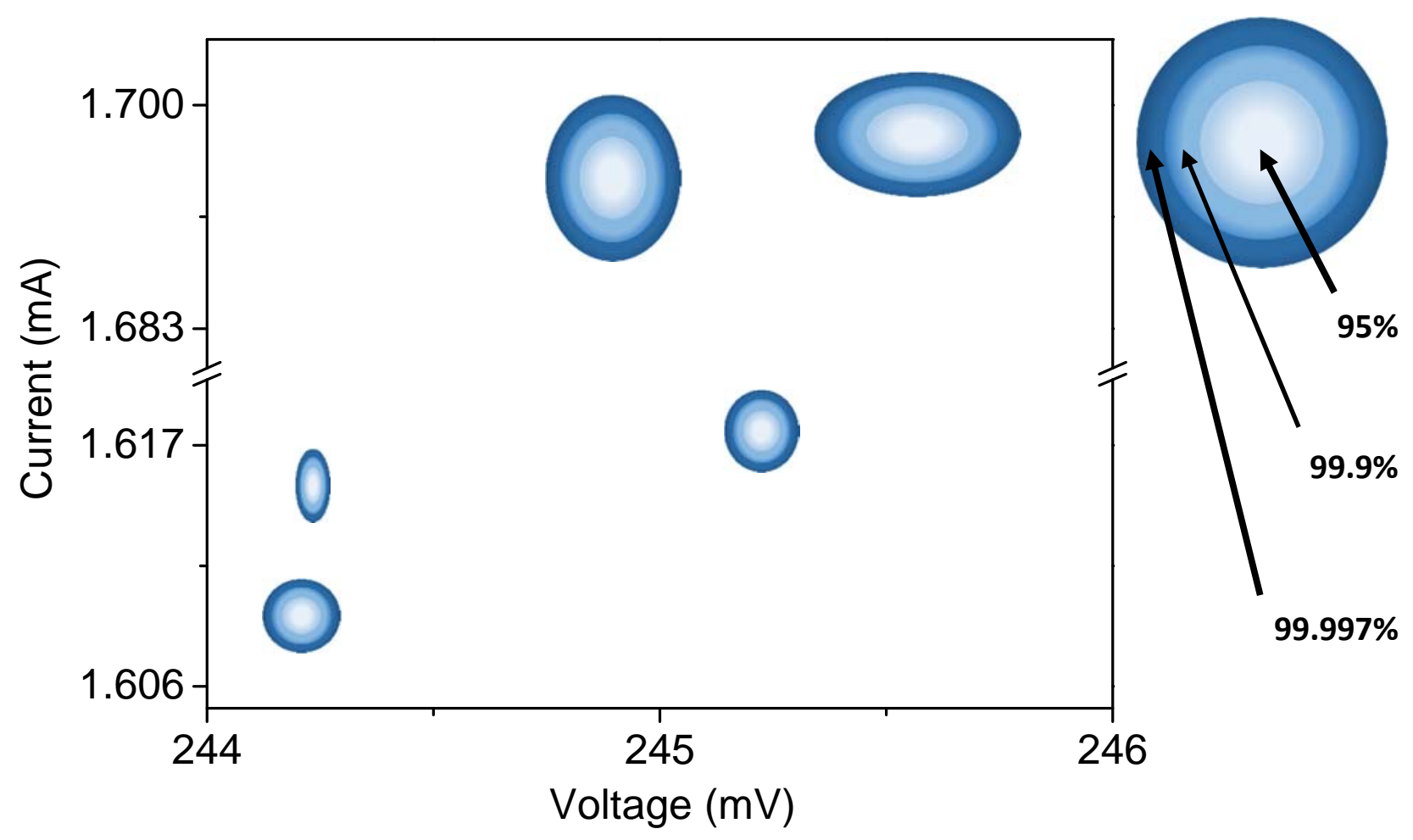

Figure 4.14: Current-voltage spectra of the highlighted region in fig 4.13 , showing the average values with their associated confidence ellipses. Ellipses were drawn by taking the standard errors in current and voltage as the semi-minor and major axes, respectively. It was found that no peak positions overlapped within a $99.997 \%$ confidence.

\subsubsection{Testing the Robustness}

The usefulness of such a device as a unique identifier is only justified if the I-V spectra can be reliably re-produced multiple times. To investigate the repeatability of RTDs, the results of the 100 spectra that was taken for the results in the previous section are shown in fig 4.15 for 4 different devices. It was observed that the position of the current-voltage peak was stable upon many measurements, and each measurement lied within 2 standard errors of the average value in both current and voltage. The data in fig 4.15 has been offset for clarity. Of course, in a realistic implementation, a single 
measurement of the device would never be used and an average value over multiple runs would always be taken but this result demonstrates the high calibre of robustness for RTDs. Furthermore, the differential current-voltage spectra has been shown in fig 4.15 , and it can be seen that this is also robust to multiple measurements. Therefore, although there is a lack of uniqueness compared to the I-V peak, the NDR region could be used as an extra layer to use for identification, if necessary. It should be noted that during some measurements, the issue of intermittent contact caused the spectra to be noisy and inconsistent. However, for the results presented these were omitted as anomalous as in a realistic implementation, the devices would have a bonded contact to the external circuitry.
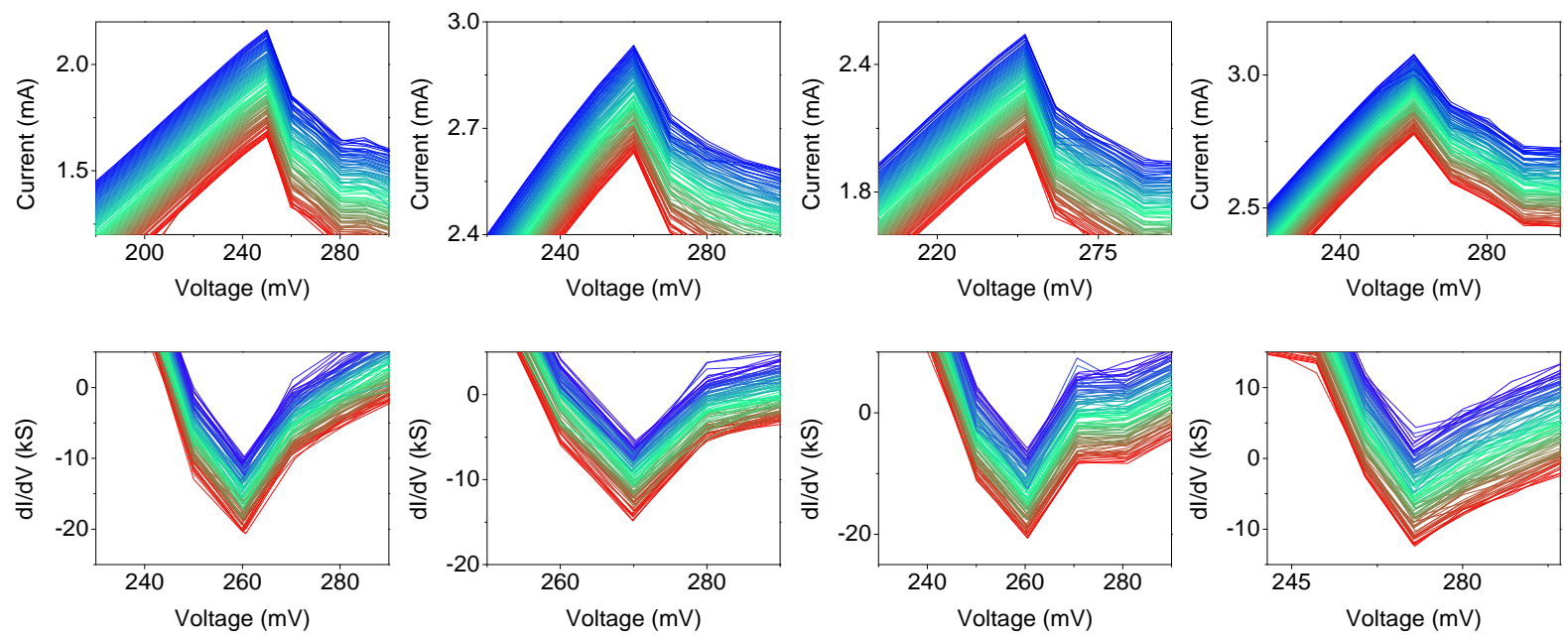

Figure 4.15: Reproducibility of the peak in the current-voltage (top row) and differential currentvoltage (bottom row) of 4 devices. It was found that the repeat of a single measurement always lied within 2 standard errors of the previously calculated average, demonstrating the high calibre of robustness for RTDs. The spectra are offset for clarity.

For an implementation setup of such a device, the current-voltage values would actually fall into bins that would correspond to a number for unique identification. The data in fig 4.13 was thus replotted in fig 4.16 to show how this would affect the robustness of such a device. This plot is a subset of an I$V$ graph that has been separated into 256 bins; the number of bins dictates the uniqueness-robustness relationship. A greater number of bins results in a more unique device but the higher possibility of it changing its identification value when re-measured and vice versa. For the data collected here, and using 256 bins, the probability that a device would fall into the wrong bin when re-measured a single time is $11.4 \%$ in the voltage domain and $0.54 \%$ in the current domain; extracted using the average 
value and 100 isolated spectra. This highlights that the current is extremely robust to re-measurement whereas the voltage bin could change values much easier. This could be corrected using error correction techniques such as fuzzy extractors, or by requiring a minimum amount of datasets to be averaged before a value is taken.

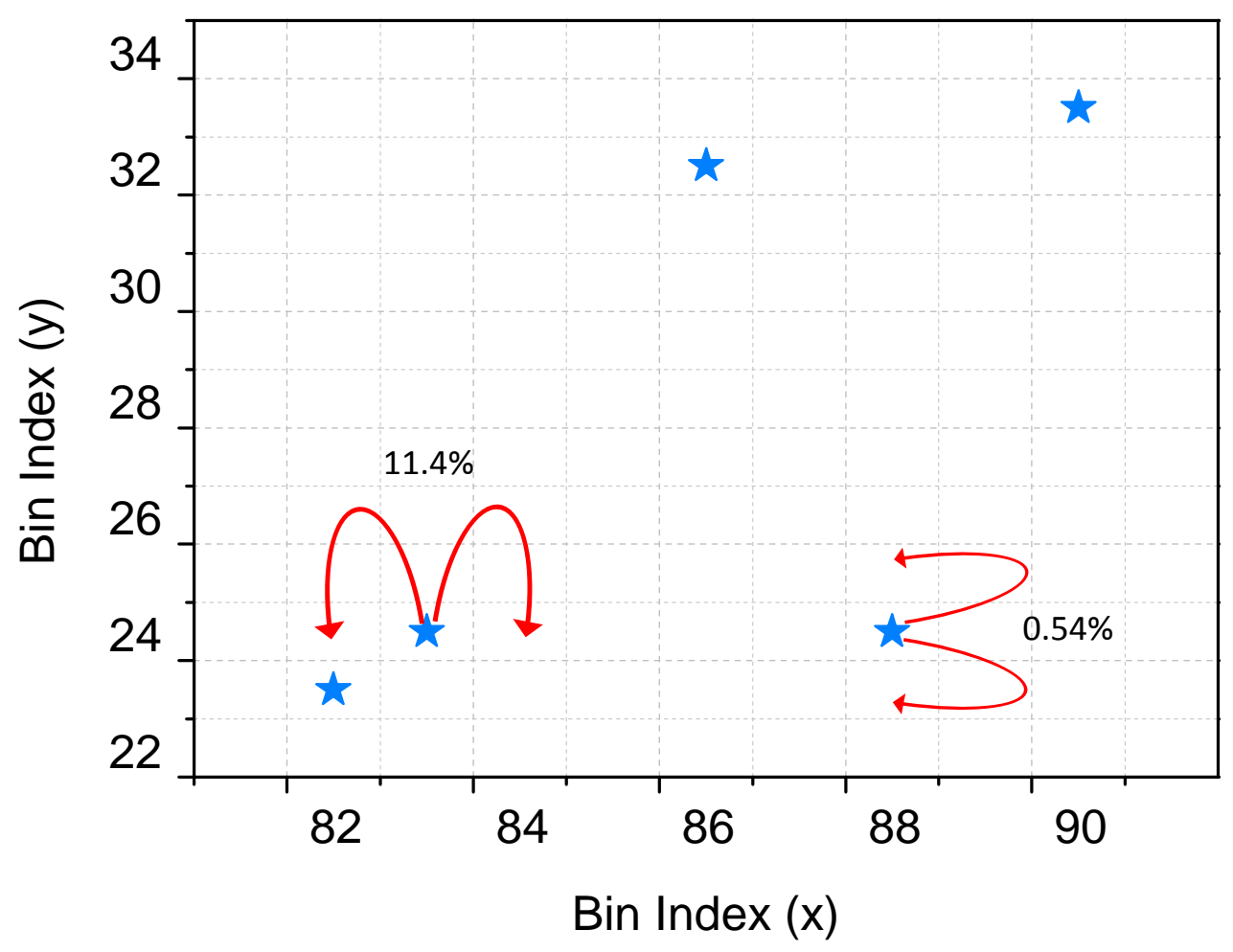

Figure 4.16: A realistic implementation would require the peak positions to fall into bins which take a range of current values, and can thus give the device a unique number. The probability of individual measurements 'jumping bins' is shown here. Where the probabilities are calculated from the number of measurements that display a deviation from the average of $>1$ bin.

\subsubsection{Implementation Considerations}

By taking the current and voltage ranges spanned here, and the average uncertainty associated with the devices, it has been calculated that on the order of $10^{3}$ unique identities could be obtained. To increase this number, various methods could be explored. Firstly, the devices could be linked together into an array, creating a circuit where the RTDs are linked either in series or in parallel, resulting in a number of peaks from a single device and thus increasing the uniqueness. Furthermore, the use of higher order nanostructures such as the quantum dots suggested in fig 4.2 could be incorporated into 
the active region. This higher number of confined energy levels would also increase the number of peaks in the I-V spectra. Here, only a single peak has been investigated whereas to obtain the maximum information content from a single device, the peak in reverse bias, valleys of the spectra and widths of the peaks could be investigated to elucidate whether or not they are unique and reproducible. These factors could increase the secure bit density past its current value of $2.5 \mathrm{bits} / \mu \mathrm{m}^{2}$, which is already twice the state-of-the-art for PUF security ${ }^{34}$.

The geometry, contact interfaces and metal thicknesses will also play a role in defining the uniqueness of a given device. However, it was reported that this effect should be small, as by using the same processing steps and simply optimising the quantum well interfaces, the I-V variations were reduced down to $1 \%$, as described in ref. [26]. Interestingly, the disruption in the growth of such a structure would make these interfaces highly distorted, which in the case of this application would be an advantage. Finally, these interfacial fluctuations could be affected through exposure to high temperatures, which would change the underlying signature and is undesirable. However, if operated in controlled conditions, this could be used to reconfigure the device and give a new fingerprint if the signature is accidentally revealed.

\subsection{Summary}

In this chapter, the derivation of an electronic unique identity from an underlying atomic structure has been shown for the first time. Such a device would provide unparalleled security due to the increased difficulty of cloning. To make use of the sensitive quantum effects arising from the varying atomic make-up, resonant tunneling diodes were employed as they provide a straightforward measurement of a macroscopic property that depends on the underpinning structure. It was proposed that the peak that arises from a single resonant tunneling diode will have a position that depends upon the internal structure on the atomic-scale, as this effects the confined state within. A chip containing many double-barrier, quantum-well resonant tunneling diodes was taken and the measurement protocol was investigated and optimised. A number of parameters were changed such 
that the measurement of an individual device could be reproduced with the highest precision. Using these conditions, 26 devices that were fabricated with a seemingly identical quantum-well, doublebarrier structure and device geometry (namely $2 \times 2 \mu \mathrm{m}^{2}$, connected via a $3 \mu \mathrm{m}$ air bridge) were explored. It was found that each of these devices resulted in a unique peak position in both voltage and current, when 100 different spectra were averaged. These devices did not overlap one another within a precision of $99.997 \%$, and thus could be used as a unique identifier. However, it was shown that the $\mathrm{dl} / \mathrm{dV}$ spectra did not display as much uniqueness, suggesting the NDR region is uniform wherever the position of the peak, so this was not used for identity extraction. Furthermore, remeasuring the peaks from individual devices gave repeatable results where the peak position of each measurement always fell within 2 standard errors of the average. When plotted on axes representing a bin number, it was shown that the re-measurement of such a device would result in probabilities of a device 'jumping' bins of $11.4 \%$ and $0.54 \%$ for voltage and current, respectively. This highlighted the need for a large number of measurements to be taken and then averaged for an accurate measurement. Also, the $\mathrm{dl} / \mathrm{dV}$ spectra resulted in robust measurements, so it was suggested that it could be used for an additional layer of identification even though it is not able to uniquely devices on its own. Using the spread of results, it was estimated that approximately $10^{3}$ unique identities could be extracted using devices of this sort and methods of increasing this number were discussed. Finally, a value of $2.5 \mathrm{bits} / \mu \mathrm{m}^{2}$ was calculated for the secure bit density, twice that of the state-of-the-art in this field.

To increase the security of the RTD devices suggested here, a number of methods can be applied to increase the amount of unique information that can be extracted. Firstly, the connection of RTDs, in series or in parallel, can result in the I-V spectra containing more than 1 peak. A result of the different RTDs coming into resonance at different voltages. Higher order nanostructures can also be employed, by using quantum dots instead of quantum wells, this will result in a higher number of energy levels being stored in the RTD. Consequently, this will result in a larger number of unique peaks in the I-V spectrum. Also, it of interest for implementation to embed the devices in a circuit architecture, this 
can be done by bonding RTDs to a chip that contains the required circuitry to measure the resonance. Longevity, temperature and other environmental factors need to be studied for the device to operate in a practical setting. Although the RTDs I-V characteristic will show changes with increasing temperature, for example, what is proposed here is a simple proof of principle that shows that such a quantum device can be employed for this application. However, it should be straightforward to calibrate such changes and this could even add another element of security to the device, by using the local temperature of the operator as another variable that can be introduced when measuring the RTD, as this value will be unknown to a remote attacker. The above will all be explored in future work. 


\section{Bibliography}

1 “Quantum Confinement: Nanoscale Materials, Devices, and Systems", M. Cahay, J. P. Leburton, D. J. Lockwood \& S. Bandyopadhyay, The Electrochemical Society, New Jersey (1997).

${ }^{2}$ R. Nötzel et al., "Uniform Quantum-dot Arrays Formed by Natural Self Faceting on Patterned Substrates", Nature, 392, 56 (1998).

${ }^{3}$ G. Juska et al., "Towards Quantum-dot Arrays of Entangled Photon Emitters", Nat. Photon., 7, 527 (2013).

${ }^{4}$ A. Mohan et al., "Polarization-entangled Photons Produced with High-symmetry Site-controlled Quantum Dots", Nat. Photon., 4, 302 (2010).

${ }^{5}$ M. Ramsteiner et al., "Influence of Composition Fluctuations in Al(Ga)As Barriers on the Exciton Localization in Thin GaAs Quantum Wells", Phys. Rev. B., 55, 5239 (1997).

${ }^{6}$ M. Tsuchiya \& H. Sakaki, "Dependence of Resonant Tunneling Current on Well Widths in AlAs/GaAs/AlAs Double Barrier Diode Structures", Appl. Phys. Lett., 49, 88 (1986).

7 D. Gammon et al., "Fine Structure Splitting in the Optical Spectra of Single gaAs Quantum Dots", Phys. Rev. Lett., 76, 16 (1996).

${ }^{8}$ R. J. Young et al., "Inversion of Exciton Level Splitting in Quantum Dots", Phys. Rev. B., 72, 113305 (2005).

${ }^{9}$ A. Gruber, "Scanning Confocal Optical Microscopy and Magnetic Resonance on Single Defect Centres", Science, 276, 5321 (1997).

10 "Doping in III-V Semiconductors", E. F. Schubert, Cambridge University Press (1993).

${ }^{11}$ W. Pötz \& Z. Q. Li, "Imperfections and Resonant Tunneling in Quantum-well Heterostructures", Solid State Electronics, 32, 12 (1989).

12 J. J. Pla et al., “A single-atom Electron Spin Qubit in Silicon”, Nature, 489, 541 (2012).

${ }^{13}$ W. Nakwaski, "Thermal Conductivity of Binary, Ternary, and Quaternary III-V Compounds", J. Appl. Phys., 64, 159 (1988).

14 "Theory of Defects in Semiconductors", D. A. Drabold \& S. Estreicher, Springer, Berlin (2007).

${ }^{15}$ E. P. Smakman et al., "GaSB/GaAs Quantum Dot Formation and Demolition Studied with Cross-sectional Scanning Tunneling Microscopy", Appl. Phys. Lett., 100, 142116 (2012).

${ }^{16}$ L. G. Wang et al., "Size, shape, and stability of InAs Quantum Dots on the GaAs(001) Substrate", Phys. Rev. B., 62, 1897 (2000).

17 N. J. Orfield et al., "Correlation of Atomic Structure and Photoluminescence of the Same Quantum Dot: Pinpointing Surface and Internal Defects That Inhibit Photoluminescence", ACS Nano, 9, 1 (2015).

18 “Complete Guide to Semiconductor Devices", K. N. Kwok, John Wiley and Sons Ltd. (2010).

${ }^{19}$ V. J. Goldman et al., "Observation of Intrinsic Bistability in Resoannt Tunneling Structures”, Phys. Rev. Lett., 58, 1256 (1987).

${ }^{20}$ T. C. L. G. Sollner, "Comment on 'Observation of Intrinsic Bistability in Resoannt Tunneling Structures'”, Phys. Rev. Lett., 59, 1622 (1987). 
${ }^{21}$ P. Zhao et al., "Simulation of Resonant Tunneling Structures: Origin of the I-V Hysteresis and Plateau-like Structure", J. Appl. Phys., 87, 1337 (2000).

22 M. A. M. Zawawi et al., "Fabrication of Sub-micrometer InGaAs/AIAs Resonant Tunneling Diode Using a Trilayer Soft Reflow Technique with Excellent Scalability", IEEE Transactions on Electron Devices, 61, pp. 23382342 (2014).

${ }^{23}$ V. A. Wilkinson et al., "Tunnel Devices Are Not Yet Manufacturable", Semicond. Sci. Technol., 12, pp. 91-99 (1997).

${ }^{24}$ M. J. Kelly, “New Statistical Analysis of Tunnel Diode Barriers”, Semicond. Sci. Technol., 15, pp. 79-83 (2000).

25 P. Dasmahapatra et al., "Thickness Control of Molecular Beam Epitaxy Grown Layers at the 0.01-0.1 Monolayer Level”, Semicond. Sci. Technol., 27, 085007 (2012).

${ }^{26}$ C. Shao et al., "Achieving Reproducibility Needed for Manufacturing Semiconductor Tunnel Devices", Electronic Letters, 49, 10 (2013).

${ }^{27}$ M. Missous et al., "Extremely Uniform Tunnel Barriers for Low-cost Device Manufacture", IEEE Electron Device Letters, 36, 6 (2015).

${ }^{28}$ M. J. Kelly, "The unacceptable Variability in Tunnel Current for Proposed Electronic Device Applications", Semicond. Sci. Technol., 21, L49-L51 (2006).

${ }^{29}$ A. Tchegho et al., "Scalable High-current Density RTDs with Low Series Resistance", IEEE International Conference on Indium Phosphide \& Related Materials (2010).

${ }^{30}$ K. J. P. Jacobs et al., "A Dual-pass High Current Density Resonant Tunneling Diode for Terahertz Wave Applications", IEEE Electron Device Letters, 36, 12 (2015).

31 "Failure Analysis A Practical Guide for Manufacturers of Electronic Components and Systems", M. Bâzu \& Titu Băjenescu, John Wiley \& Sons (2011).

32 S. F. Nafea \& A. A. S. Dessouki, "An Accurate Large-signal SPICE Model for Resonant Tunneling Diode”, IEEE International Conference on Microelectronics (2010).

${ }^{33}$ W. Lian, "Resonant Tunneling Diode Mixer and Multiplier", Simon Fraser University Thesis (1994).

34 "Physically Unclonable Functions", R. Maes, Springer, Berlin (2013). 


\section{Chapter 5}

\section{Langmuir-Blodgett Deposition of 2D Materials for Unique Identification}

\subsection{Introduction}

There are many instances in the field of security where optically identifiable tags are used, examples of these include holograms, special inks/prints and conventional anti-tamper taggants. Unfortunately, there are a range of problems with these existing systems, none more so than their ease of clonability and lack of individuality. Thus, it is of great societal and technological significance to develop an optically addressable analogue of an UNO/PUF-like device that can overcome these problems by relying on quantum confinement and thus, the local atomic environment of the system. In this chapter, the possibility of this is suggested by using two-dimensional materials known as transition metal dichalcogenides (TMDs), which contain a direct band-gap in the visible range and therefore emit light that could be detected efficiently by a standard silicon CCD. Although individual monolayers have outstanding properties, their size limits potential uses, and for a practical realisation of a 2D material based, optical identification tag, a method of macroscopically depositing 2D materials is needed. The emphasis of this chapter is therefore on the creation of TMD devices using the Langmuir-Blodgett (LB) technique, for the first time. Specifically, large-area films of $\mathrm{MoS}_{2}$ have been fabricated, and has provided a facile method of creating macroscopic films of 2D materials. This method has thus far been used sparingly for 2D materials, limited to the deposition of graphene and graphene oxide. It is shown in this work that by utilising electrospray for deposition onto the air-water interface, the creation of thin films of effectively any 2D material could be achieved. 


\subsection{Unique Properties of 2D Materials}

\subsubsection{An Introduction to 2D Materials}

The rise of two-dimensional materials dates back to 2004, when Novoselov et al. isolated and characterised graphene sheets ${ }^{1}$. Graphene is an allotrope of carbon that comprises an atomically thin honeycomb lattice of $\mathrm{sp}^{2}$ hybridised atoms that can separated from bulk graphite due to the weak Van der Waals force between the layers.

Graphene has exceptional in-plane electrical, mechanical and thermal properties such as having an electron mobility of $200,000 \mathrm{~cm}^{2} \mathrm{~V}^{-1} \mathrm{~s}^{-1}$ and a Young's modulus of approximately $2.4 \mathrm{TPa}^{2,3}$. These interesting properties arise due to graphene's unique band structure and the effect of confining electrons and holes in 0, 1 and 2 dimensions ${ }^{4,5,6}$.

Since the discovery of graphene, there has been a plethora of investigations into other twodimensional materials that can also be separated from their bulk counterparts due to weak interplanar Van der Waals forces. Examples of these include, but are not limited to, the transition metal dichalcogenides (TMDs), such as $\mathrm{MoS}_{2}$ and $\mathrm{WS}_{2}{ }^{7,8}$. These materials are of great interest in the field of optics, as their band structure undergoes evolution from an indirect-gap to a direct-gap semiconductor as the number of layers in the sheet is reduced to a single layer ${ }^{9}$. It is these materials in particular that will be the topic of discussion in the following chapter for creating an optical security device. $\mathrm{MoS}_{2}$, specifically, is of great interest as it emits in the near infrared and thus takes advantage of a standard silicon CCDs enhanced sensitivity in this range.

\subsubsection{Effects of Confinement in 2D Materials}

As aforementioned, the confinement of charge carriers in two-dimensional materials gives rise to interesting properties. These properties can be used in a range of applications although it is often required to have high-quality monolayer sheets. However, although two-dimensional materials are often displayed as being atomically perfect, it has been observed that imperfections arise in these materials and play a role in effecting the properties of the material ${ }^{10,11,12,13,14}$. These atomic variations 
in the $2 \mathrm{D}$ material can lead to significant differences in the wavelength of any emitted light, a macroscopic property that is easy to measure optically. This promises a simple means to visually verify the identity of an object with a 2D material on it, as illustrated in fig 5.1a. Furthermore, a large family of $2 \mathrm{D}$ materials now exists (see fig 5.1b), with new materials continually emerging, allowing this concept to be expanded across the entire visible range; using multiple materials also provides a method of enhancing the security of the system ${ }^{15}$.
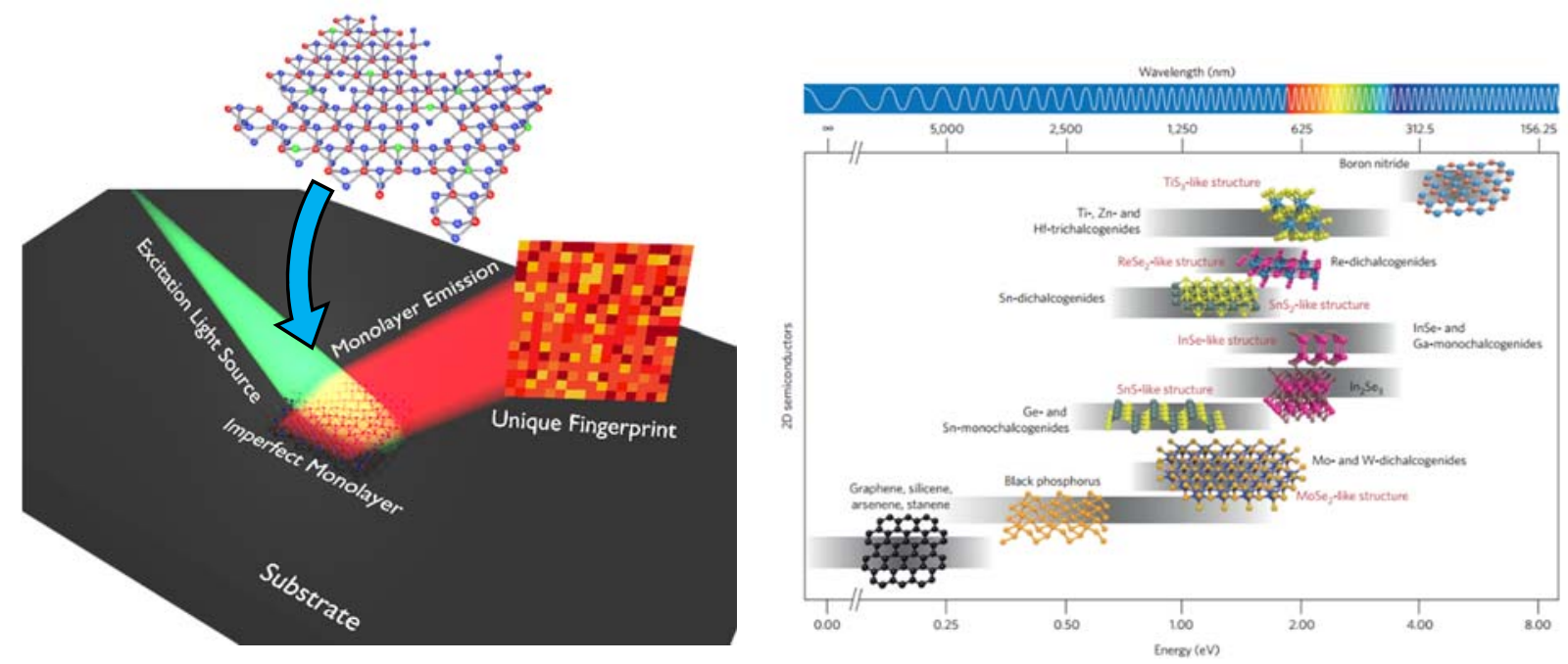

Figure 5.1: (a) Imperfections such as dangling edges, vacancies, and substitutions present in 2D materials could be used as an optically active unique identity. For example, measuring wavelength and intensity shifts across a monolayer, could enable the extraction of a unique fingerprint with unrivalled security, (b) current family of 2D materials, spanning the visible range could enhance the efficiency and security even further by coupling materials together. [(b) reprinted with permission from Macmillan Publishers Ltd: from [15], copyright (2016)].

To elaborate on the optical effect of these imperfections, a 2D flake was mechanically exfoliated onto $\mathrm{SiO}_{2}$ (Fig 5.2a and b). Here, a bulk WSe $e_{2}$ crystal was placed onto a residue-free piece of tape and continuously cleaved by pressing the crystal onto clean areas of tape. The thickness of the 2D sheets reduces with each cleave and once adequately cleaved can be transferred onto $\mathrm{SiO}_{2}$ for isolation of an individual layer. Prior to transfer, the $\mathrm{SiO}_{2}$ was cleaned in acetone and IPA for 5 minutes each before being dried with $\mathrm{N}_{2}$. Following this, the $\mathrm{SiO}_{2}$ was placed in a high-power plasma asher (Diener Femto system, $98 \% / 2 \% \mathrm{Ar} / \mathrm{O}_{2}, 15 \mathrm{~L} / \mathrm{h}$ flow rate) for 5 minutes which removes any organic contaminants 
remaining on the surface. The ashing also provides a source of heating for the $\mathrm{SiO}_{2}$ substrate. The strict cleaning requirements and heating of the substrate are to promote adhesion of layers to the substrate such that it does not stay on the tape. To transfer the flakes, the ashed $\mathrm{SiO}_{2}$ substrate was removed from the asher and placed immediately onto the tape containing the 2D material and subsequently manual pressure was applied for approximately 2 minutes. To obtain the best results, the tape is then peeled away from the $\mathrm{SiO}_{2}$ substrate slowly.

An optical microscope was used for identification of individual flakes. The surface of the substrate is scanned using a 50x objective lens, importantly the substrate must contain approximately $300 \mathrm{~nm}$ of $\mathrm{SiO}_{2}$ on top of intrinsic $\mathrm{Si}$ as this maximises the optical contrast of thin flakes due to the interference of these layers with white light ${ }^{16}$. The thickest flakes appear white to yellow in colour and as the thickness is reduced the flakes appear green, blue, red and then purple due to the light constructively interfering at these wavelengths. Monolayer flakes are characterised by their semi-transparent, purple looking colour. Once an individual layer was identified, its emission properties were explored using micro-photoluminescence. Fig 5.2c illustrates the spectra obtained from a number of positions within the same bilayer flake. Optical images show that the surface of the flake is 'homogeneous' across areas 1, 2 and 4 which is best elucidated in fig 5.2b; dark-field images are collected by illuminating the sample at large angles enabling the detection of any clear surface variations or steps. The photoluminescence spectra shown in fig 5.2c demonstrate that the emission profile, intensity and wavelength can be affected by unseen local variations across the flake. The expected signal is a single delocalised neutral exciton peak at room temperature at approximately $752 \mathrm{~nm}$ and $806 \mathrm{~nm}$ for monolayer and bilayer, respectively ${ }^{17}$. It is observed that as the excitation position on the flake is changed, slight intensity and wavelength shifts are measured, whilst in some spectra a smaller shoulder is witnessed next to the main peak. Interestingly, profile 3 in fig 5.2c displays emission from an edge-like feature in the layer, which shows sharp features that are thought to arise through localised excitons; due to trapping of free excitons by defects ${ }^{18}$. From these measurements, it is clear that differences in the recombination of carriers can be measured across the surface of a 2D material. 
Similar results have been observed for other $2 \mathrm{D}$ materials, where the variations in the measured photoluminescence spectra is directly attributed to the existence of defects and impurities ${ }^{19,20}$. Furthermore, it has been shown that artificial defects can be forced into the $2 \mathrm{D}$ material which creates a larger variation in the photoluminescence wavelength and intensity. This can be done using oxygen plasma treatment of flakes, for example ${ }^{21}$. These device dependent atomic variations can be used for identification purposes through the macroscopic measurement of their emission profile. However, mechanically exfoliated flakes typically range from 1-50 $\mu \mathrm{m}$ in size and have to be identified and addressed individually. In this chapter, the fabrication of a macroscopic identity tag will be pursued using the scalable and facile Langmuir-Blodgett technique. This technique has been employed for 2D materials such as graphene but has yet to be applied to TMDs. In this work, the creation of large-area identity tags via the Langmuir-Blodgett deposition of $\mathrm{MoS}_{2}$, a 2D material that emits near infrared light, will be attempted for the first time.

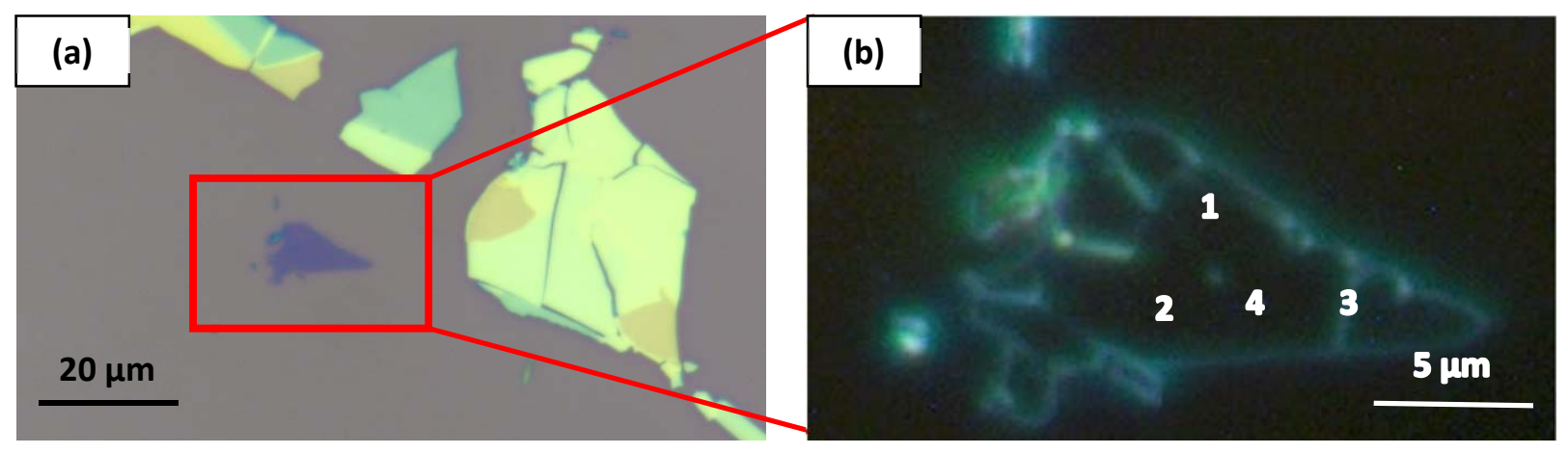

(c)
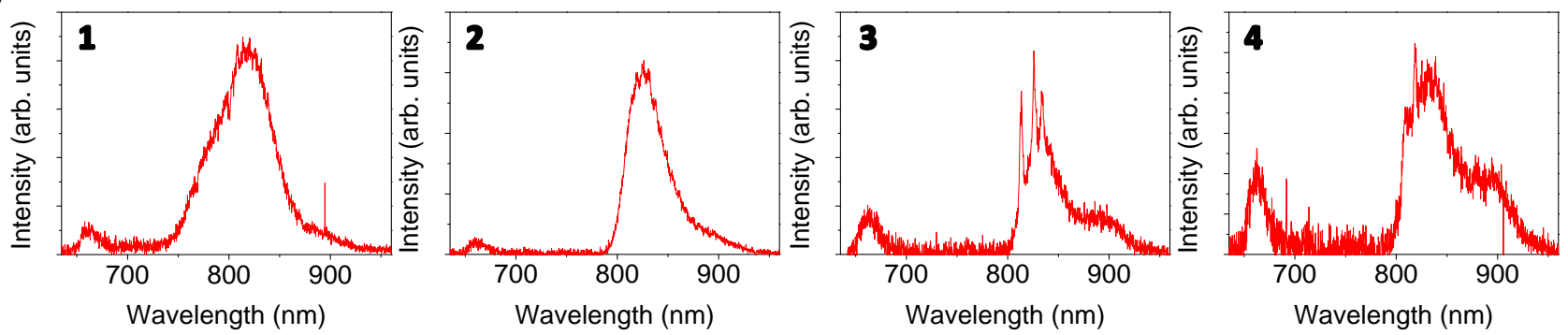

Figure 5.2: Optical images of a monolayer (a) 50x objective bright-field image, (b) 50x objective darkfield image. This shows that no observable changes are present that should affect the photoluminescence significantly. (c) photoluminescence spectra highlighting the differences that can be found by exciting different areas of a 2D material (WSe2), suggested to arise from area-dependent impurities and defects. 


\subsection{Langmuir-Blodgett Films of 2D Materials}

\subsubsection{A Brief Comparison of Fabrication Methods}

At present, the main method of fabrication of 2D materials is mechanical exfoliation, as described in section 5.2.2. This allows monolayer sheets of superior quality to be manipulated into devices in order to answer fundamental research questions. Unfortunately, the future of 2D materials does not lie with this method as there is no scope for scalability or mass production of devices. There have been a number of other methods developed for producing 2D materials in a cost effective way, albeit with consequences on their quality; the main methods are summarised in fig 5.3. Chemical vapour deposition has appeared as the most promising candidate with a large range of companies now producing graphene via this method on a large-scale. However, research into the growth of other 2D materials such as the TMDs is still in its infancy with much less research reported compared to graphene ${ }^{22}$. The most common TMDs, $\mathrm{MoS}_{2}$ and $\mathrm{WS}_{2}$, have however been grown into continuous films on $\mathrm{SiO}_{2}$ and studies into the growth of heterostructures by altering the growth conditions have been reported ${ }^{23,24}$.

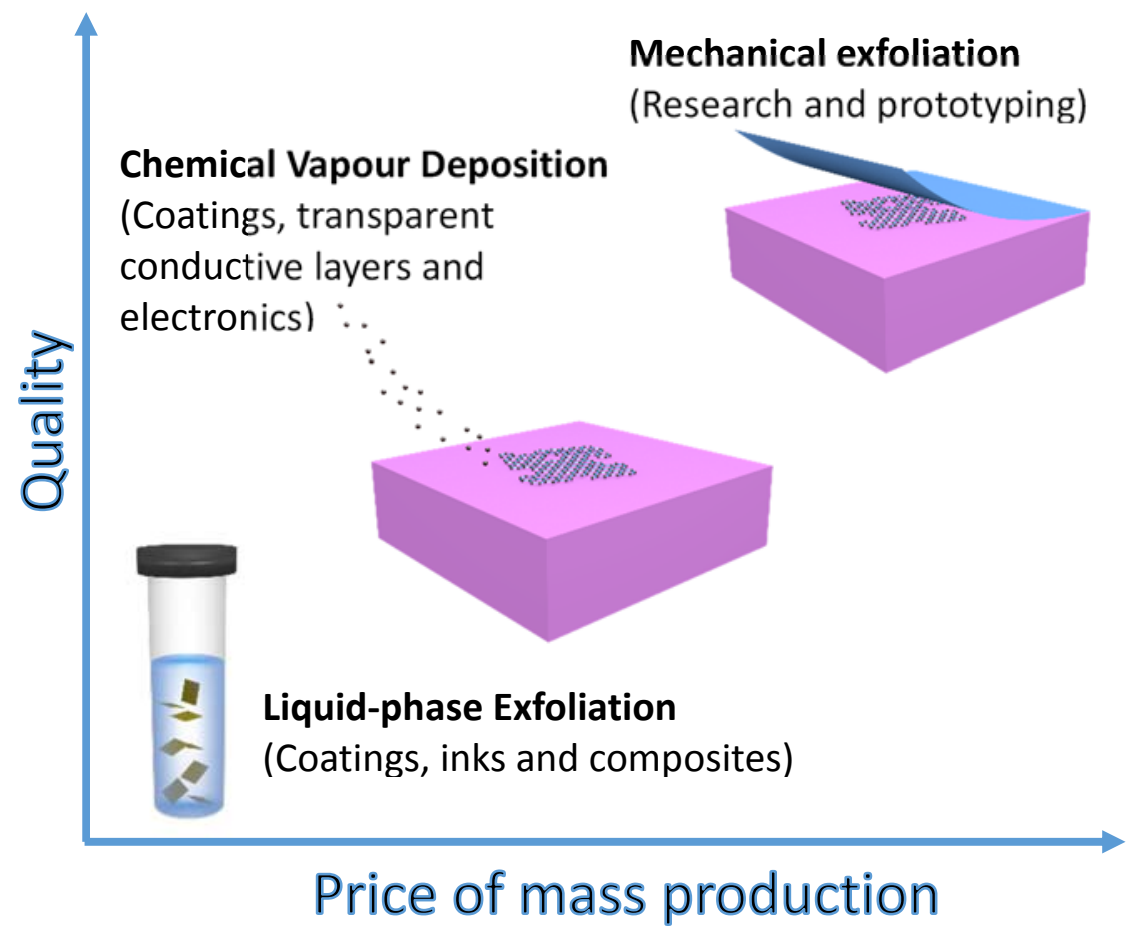

Figure 5.3: Main current production methods of 2D materials, comparing quality to scalability; liquid exfoliation of 2D materials shows scalability potential for integration via many methods. 
Another method that has risen to prominence in the last few years due to its greater scalability and ubiquity for a number of applications is liquid phase exfoliation; separating and storing monolayers of 2D materials in solution ${ }^{25}$. This area has gained much attention due to its applicability to 3D printing, monolayer inks and coatings. The 2D materials can be exfoliated in solution either by mechanical or chemical means and stored in a variety of solvents including aqueous environments without aggregating. In the best example of this method, solutions comprising $75 \%$ monolayer content have now been developed using a technique known as liquid cascade centrifugation ${ }^{26}$.

\subsubsection{Thin Films of Liquid Exfoliated 2D Materials}

Although the creation of high monolayer content dispersions have been observed, the fabrication of these solutions into monolayer films has received far less attention. A variety of methods are often employed for creating thin films of 2D materials, including; drop or dip casting, spin, rod or spray coating, vacuum filtration and screen or inkjet-printing ${ }^{27}$. Unfortunately, the lack of thickness control using these methods prevents the controllable formation of uniform monolayer thin films. In recent years, the Langmuir-Blodgett (LB) technique has been employed to fabricate thin films of 2D materials using such dispersions of liquid exfoliated nanosheets. To date, only graphene and graphene oxide have successfully been deposited via this method ${ }^{28,29}$. Furthermore, there is some historical work that has been completed on coupling TMDs in the bulk to amphiphilic molecules on the surface of water or securing them at a liquid-liquid interface for deposition ${ }^{30,31,32}$. In this work, the direct creation of Langmuir-Blodgett films of $\mathrm{MoS}_{2}$ is reported for the first time. Similar to that reported by Nie et al., the main step that was taken into achieving this was though the use of electrospray for spreading the 2D materials held in solution onto the trough, as opposed to the conventional spreading of solution (see fig 5.4) 33 $^{33}$ The LB technique provides an excellent method to fabricate macroscopic devices of $\mathrm{MoS}_{2}$, as it can facilitate deposition over an entire wafer, if necessary. Furthermore, although no other materials have yet been tested, there is no physical reason why this technique could not be expanded for the creation of thin films of any given 2D material. 


\subsubsection{Creating a Langmuir Film of $\mathrm{MoS}_{2}$}

For all the following preliminary experiments, $\mathrm{MoS}_{2}$ solution was attained from Graphene Laboratories Inc. ${ }^{34}$ and used as received. The stock solution is reported to have a concentration of $18 \mathrm{mg} / \mathrm{L}$ and a thickness distribution of 1-8 layers and is held in a mixture of ethanol and water. In all cases, the $\mathrm{MoS}_{2}$ was sonicated for 10 minutes before deposition to re-disperse any aggregates that had formed in the solution. The $\mathrm{MoS}_{2}$ solution has a characteristic brown colour and is semi-transparent, and following sonication no aggregates were visible in the solution under optical inspection. To enable the demonstration of electrospray as an effective tool for spreading $\mathrm{MoS}_{2}$, three isotherm experiments were performed, as shown in fig 5.5.
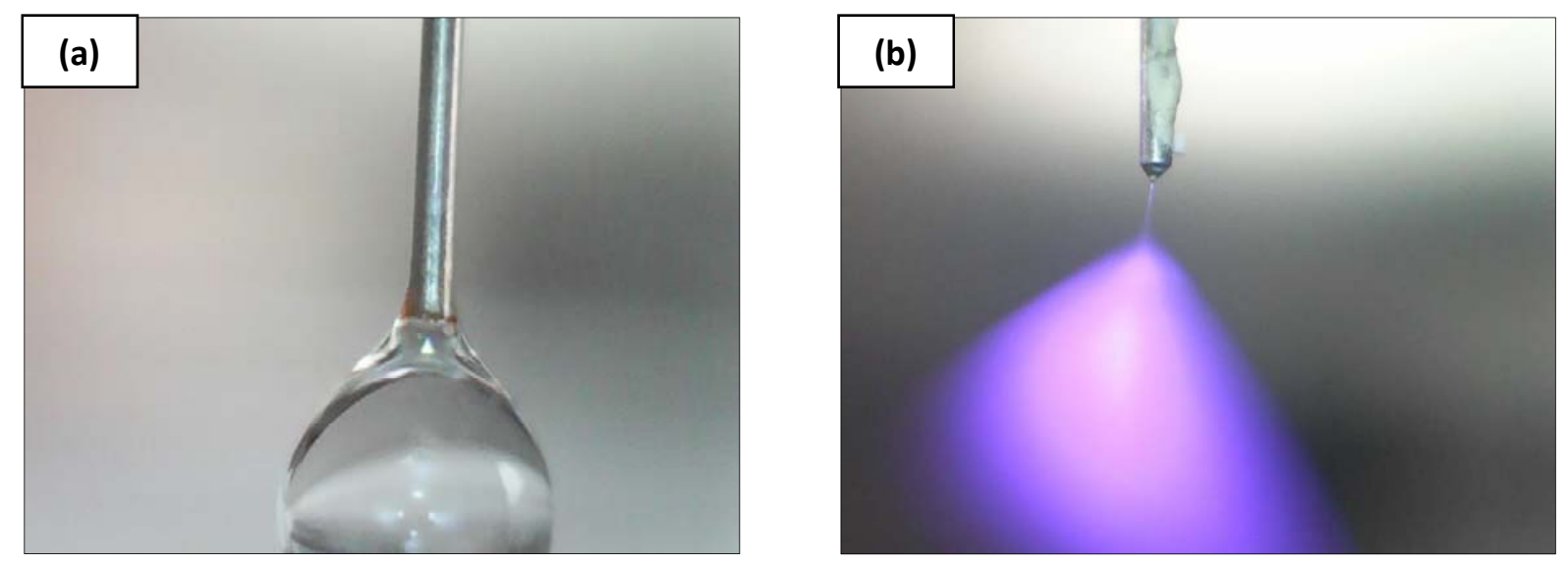

Figure 5.4: Different methods of solution deposition (a) conventional spreading with a droplet size on the order of mm's, causes lots of mixing with the subphase, (b) electrospraying spreading results in sub-micron droplet sizes and facilitates negligible mixing, even when miscible liquids are used.

An approximate expected value for the required volume of solution to create a film that contains a complete and continuous coverage of the $\mathrm{MoS}_{2}$ flakes derived from the solution is straightforward to calculate once the following assumptions are made. Taking an average layer thickness of 4 layers and assuming the solution is uniformly distributed, treating the flakes as perfect squares with an average size of $100 \mathrm{~nm} \times 100 \mathrm{~nm}$ and a thickness of $6.15 \AA$, and assuming the density of thin $\mathrm{MoS}_{2}$ flakes to be the same as that in the bulk crystal ${ }^{35}$. 
Using these assumptions the volume of an individual, 4 layer flake is calculated as $2.46 \times 10^{-23} \mathrm{~m}^{3}$, which when multiplied by the density $\left(5.06 \times 10^{9} \mathrm{mg} \mathrm{m}^{-3}\right)$ gives each flake a mass of $1.24 \times 10^{-13} \mathrm{mg}$. The minimum area that the barriers can be compressed to without clashing with the Wilhelmy plate is approximately $35 \mathrm{~cm}^{2}$, dividing this by the lateral area that each flake occupies $\left(1 \times 10^{-14} \mathrm{~m}^{2}\right)$ gives the number of flakes required to fill the available surface area, calculated as $3.5 \times 10^{11}$. Thus the total mass of flakes required is $0.0434 \mathrm{mg}$, dividing this by the concentration of the solution $(18 \mathrm{mg} / \mathrm{L})$ results in an approximate starting volume of $2.41 \mathrm{ml}$. Thus, for the following experiments a starting volume of $2.5 \mathrm{ml}$ was used.

Firstly, $2.5 \mathrm{ml}$ of ethanol was dropped onto the surface of the water using the conventional spreading method such that droplet diameters were on the order of mm's (see fig $5.4 \mathrm{a}$ ), in this case a $0.5 \mathrm{ml}$ syringe was used. Once deposited, any remaining ethanol on the surface was given 30 minutes to evaporate so that there was no solvent remaining to skew the results. The barriers were then symmetrically closed at a rate of $5 \mathrm{~mm} / \mathrm{min}$ whilst the pressure was recorded with the tensiometer. The black trace in fig 5.5 shows the result, it was observed that although ethanol should produce a flat isotherm as there should be nothing on the surface, there was in fact a slight pressure increase as the barriers closed to very small areas. This has been attributed to the ethanol itself containing contaminants, with a volume of $2.5 \mathrm{ml}$ containing enough to see a measurable change at small areas. This result provided a good control measurement for the subsequent experiments.

Next, the entire trough was emptied, re-cleaned and prepared and $2.5 \mathrm{ml}$ of the stock $\mathrm{MoS}_{2}$ solution was taken and conventionally dropped onto the water surface using the same syringe and methodology. To minimise direct mixing, the deposition of droplets is done in a controlled manner such that they are formed at the end the needle before being moved slowly towards the surface which then 'pulls' the droplet onto the surface. As before, the solution was allowed 30 minutes to evaporate such that only material should be left on the water surface and then the barriers were closed at the same speed of $5 \mathrm{~mm} / \mathrm{min}$ whilst the pressure was recorded. 
The red trace in fig 5.5 presents this outcome, it was seen that although the deposited solution now contains $\mathrm{MoS}_{2}$ flakes, the pressure-area isotherm showed a very similar curve. This measurement in itself lends support to the theory that using a water-miscible solvent such as ethanol results in a lot of mixing with the water subphase as no pressure rise is observed until low areas are reached. The discrepancy in results between dropped $\mathrm{MoS}_{2}$ and ethanol is likely due to two factors, the first being that the $\mathrm{MoS}_{2}$ solution will contain a larger density of contaminants due to the nature of the chemical process required to create the solutions, whilst the second is that a small amount of the $\mathrm{MoS}_{2}$ will stay on the surface during spreading. However, as the shape of the isotherm is very similar to that of ethanol, the increased number of contaminants is expected to be the main contributing factor to this offset. To further investigate whether a large percentage of $\mathrm{MoS}_{2}$ remains on the surface, the water surface can be inspected optically as the $\mathrm{MoS}_{2}$ has a brown hue, fig 5.6a shows that there is no observable colour difference between the inside and outside of the barriers further suggesting that a large amount of mixing has taken place.

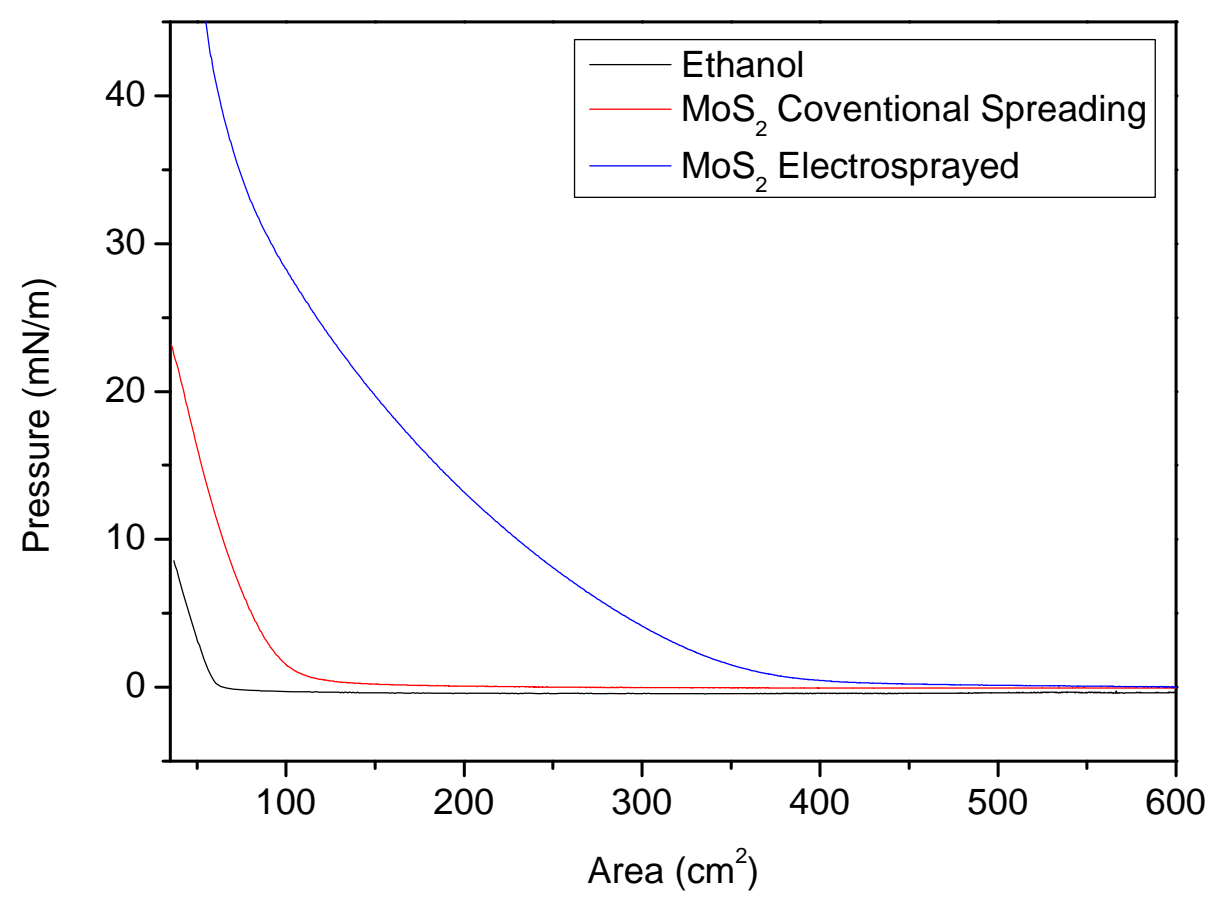

Figure 5.5: Pressure-area isotherms demonstrating the effect of electrospraying $\mathrm{MoS}_{2}$ onto the LB trough as opposed to conventional spreading. The black and red trace show the conventional spreading of ethanol and $\mathrm{MoS}_{2}$ (in ethanol), respectively. The blue trace shows the same $\mathrm{MoS}_{2}$ solution that has been electrosprayed, resulting in a significant change in the isotherm. 
The pressure-area experiment was then repeated a third time after re-preparing the trough but in this case, $2.5 \mathrm{ml}$ of the $\mathrm{MoS}_{2}$ stock solution was electrosprayed onto the surface. For this experiment, a stainless steel dispensing needle with a $100 \mu \mathrm{m}$ diameter (attained from Nordson $\mathrm{EFD}^{36}$ ) was attached to a $5 \mathrm{cc}$ syringe barrel via a lure lock, and held at approximately $5 \mathrm{~cm}$ above the surface. The needle was connected to a current limited high-voltage power supply, whilst a copper plate is placed in the water and grounded. As the ratio of water to ethanol is unknown, it is hard to predict a turn on voltage for the spray. Therefore, the voltage was slowly increased whilst focussing on the tip of the needle with a USB endoscope and illuminating the needles tip with a UV torch in order to increase contrast of the solution droplets. When a stable spray comprising a Taylor cone, a small jet and then a nebulised spray (cone-jet mode) can be seen on the image (see fig 5.4b), the voltage was kept at this value.

In the case of this particular experiment, the voltage was approximately $3.5 \mathrm{kV}$. It should be noted here that no external pressure is applied to achieve the electrospray dynamics and the system solely relies on the electric pressure alone. Due to the small radius of the dispensing needle, the flow rate in this experiment was very low, requiring approximately 6 hours to finish spraying. Furthermore, due to the lack of external pressure, there is always an unavoidable volume of solution left in the $5 \mathrm{cc}$ syringe barrel. After the completion of spraying, the trough was left for 30 minutes before compressing the barriers at a rate of $5 \mathrm{~mm} / \mathrm{min}$. The data gathered here is shown in the blue trace in fig 5.5 . It was instantly observed that the final pressure is more than double that of the conventionally spread $\mathrm{MoS}_{2}$, and perhaps more importantly displayed a different shape in the isotherm. The shape of an isotherm varies when different molecules are compressed on a water surface, so this observation suggests there is something other than just contaminants on the surface; although these will still be present in the film. Also, the gradient of the isotherm is almost vertical at low areas, indicating the approach of a solid phase on the water surface, as anticipated by the initial calculations, though the arrival of a critical pressure was never observed. 
Furthermore, the rise in pressure begins at much larger areas, suggesting that sheets on the surface are beginning to interact with one another much earlier. In summary, it is evident that electrospray has provided a means to organise a Langmuir film containing $\mathrm{MoS}_{2}$ on the surface of water. This is further elucidated by inspecting fig 5.6b, which shows how the colour of the water in between the barriers is the same as the starting solution, in stark contrast to that observed in fig 5.6a. Clearly, if there was ample mixing taking place in this experiment then a lack of colour difference as in fig 5.6a would be expected, further suggesting that a large percentage of the $\mathrm{MoS}_{2}$ remains on the surface during electrospray.
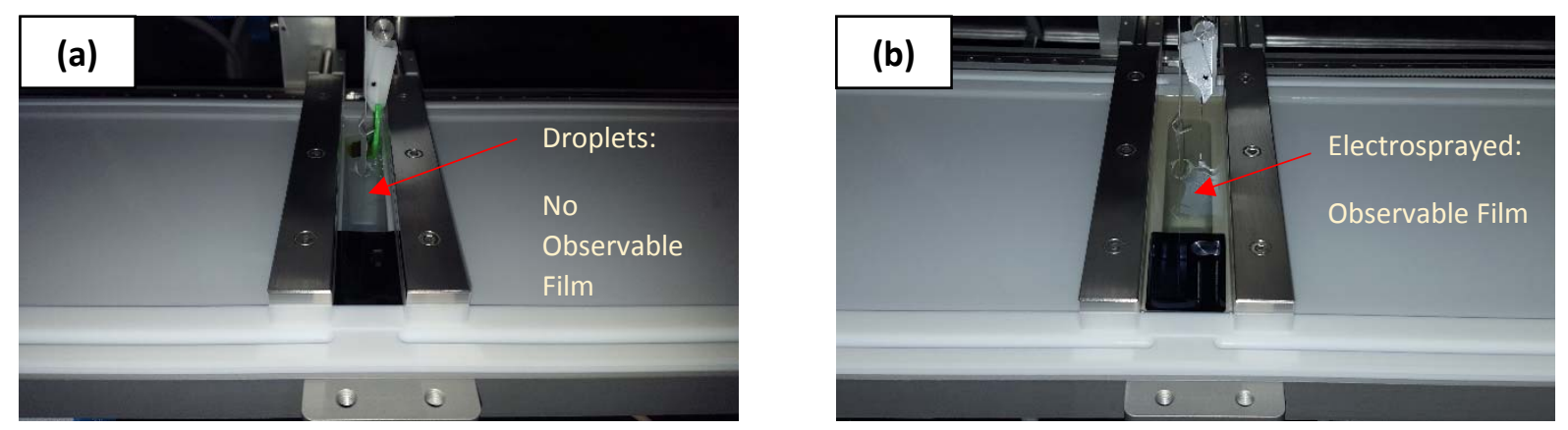

Figure 5.6: Images of a fully compressed film between two barriers when (a) conventionally spread and (b) electrosprayed. This shows clear evidence for the existence of a Langmuir film when electrospray is employed.

Finally, it is expected that some $\mathrm{MoS}_{2}$ flakes are undoubtedly lost to the subphase in the third experiment. This becomes especially evident at high pressures when if the barriers are kept at a constant position, a steady drop is seen to an equilibrium pressure. This could be explained by the flakes compressing so that they interact strongly with themselves and contaminants, forcing them to go either over or under sections of the film, leading to some being forced into the subphase. However, this is still under investigation as another possible mechanism could be the flakes being adsorbed onto the Wilhelmy plate and the barriers, an effect which should be enhanced at higher pressures. 


\subsection{4. $\mathrm{MoS}_{2}$ at the Air-Water Interface}

To investigate how the $\mathrm{MoS}_{2}$ flakes behave after being placed onto the surface via electrospray, an oscillating barrier experiment was performed. Here, the trough was prepared and $1 \mathrm{ml}$ of the stock $\mathrm{MoS}_{2}$ solution was electrosprayed onto the surface $(\sim 5 \mathrm{~cm}, 3.5 \mathrm{kV})$ in the cone-jet mode. Subsequently, the Brewster angle microscope (BAM) was placed into position over the black glass and the barriers were set to oscillate back and forth between a barrier position of $0 \mathrm{~mm}$ (fully open) and $239 \mathrm{~mm}$ (closed) for a total of 40 oscillations. The final area here is approximately $82 \mathrm{~cm}^{2}$, as the barriers could not be fully closed in this experiment as the BAM was being used and takes up approximately $5 \mathrm{~cm}$ width on the water surface. The results of the pressure-area isotherms recorded during the oscillating experiment can be seen in fig 5.7. It was observed that the film showed hysteresis during each compression-expansion cycle. This effect was highlighted in the inset to fig 5.7 which looks at the cycles in more detail by applying an offset, it is evident that the hysteresis is becoming exaggerated after each cycle. However, the flaky domains become negligible in the pressure measurement as the available area becomes larger, which is why the pressure is able to return to zero each time. As the observed hysteresis rises with more cycles, it is thought that more flakes begin to coalesce to form larger regions after each cycle, making it harder for the film to return to its previous state.

Interestingly, after each compression, a small pressure drop was measured. This is thought to be due to the barriers being held at the closed position for 30 s before re-expansion, resulting in a pressure drop via a mechanism described in the previous section. However, the total pressure drop is only 3-4 $\mathrm{mN} / \mathrm{m}$, which could be explained by the removal of contaminants to the subphase (the pressure of $1 \mathrm{ml}$ of ethanol would rise to approximately $4 \mathrm{mN} / \mathrm{m}$ using the data in fig 5.4). The removal of contaminants is also supported by the final couple of cycles in fig 5.7 (red traces), here the individual compression and expansion isotherms seem to overlap extremely well compared to that of previous cycles. This could be explained through the removal of the largest parts of the film (contaminants) which can upset the film and amplify material loss into the subphase, causing pressure losses. 
The Brewster angle microscopy images of the water surface during compression are shown in fig 5.8. These images were taken during the electrospray experiment from fig 5.4 and included here to give the best insight about high pressure behaviour. The BAM images were taken every 30 s once the pressure had risen beyond $1 \mathrm{mN} / \mathrm{m}$, this corresponds to a decrease in area of $\approx 40 \mathrm{~cm}^{2}$ per image, calculated using the rate of the barriers $(5 \mathrm{~mm} / \mathrm{min})$. Included as a reference, fig 5.8 a shows a typical BAM image of a clean water surface, where there is evidently no material. Although the BAM images should give a representative snapshot of how the film is behaving on the water surface, it should be noted that it only observes $0.015 \%$ of the total area of the film. Thus, it is possible that what is observed is dependent on the locality of the BAM head but it is presumed here that the overall film characteristics can be captured by assuming there is no special area in the film that displays different traits. The images shown in fig $5.8(b-d)$ show how the film behaves at relatively low pressures where not much of the surface is taken up by material.

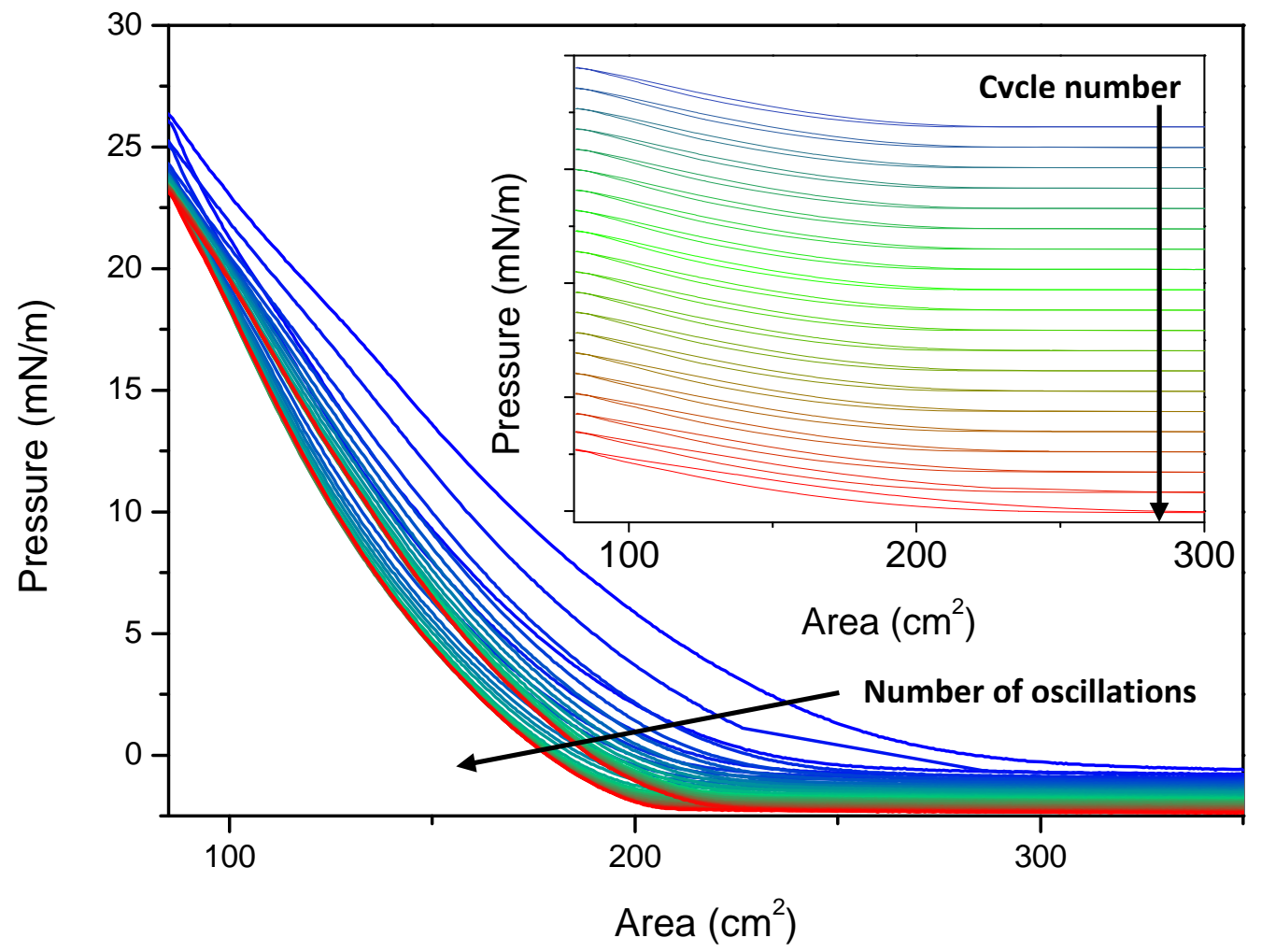

Figure 5.7: An oscillating barrier experiment of $\mathrm{MoS}_{2}$ at the air-water interface probing the surface dynamics. The barriers are moved back and forth a total of 40 times, resulting in hysteresis due to coalescing of the floating film. Inset: Compression-expansion cycles with offset for clarity, blue -> red indicates compression-expansion cycle number 1 -> 20. 

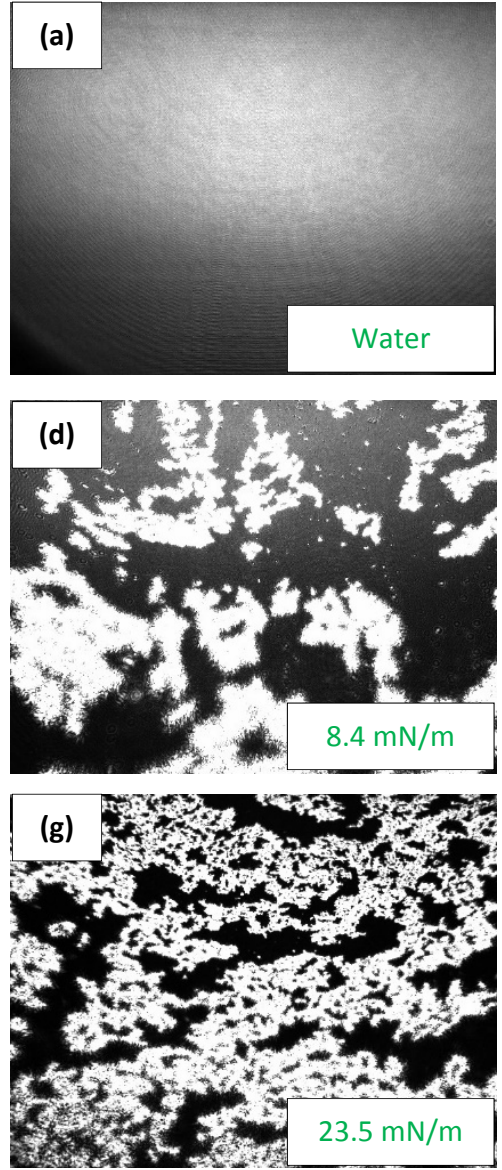
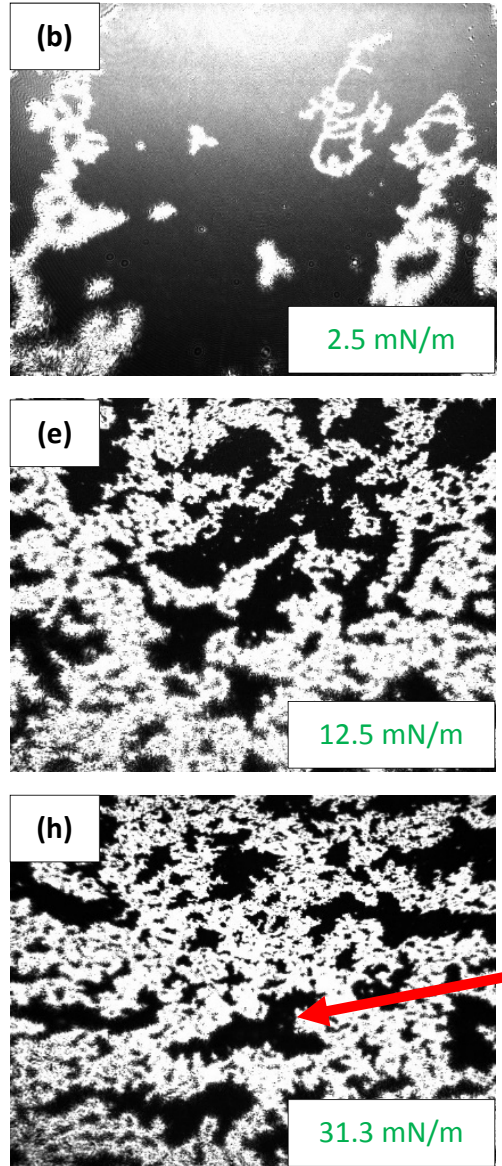
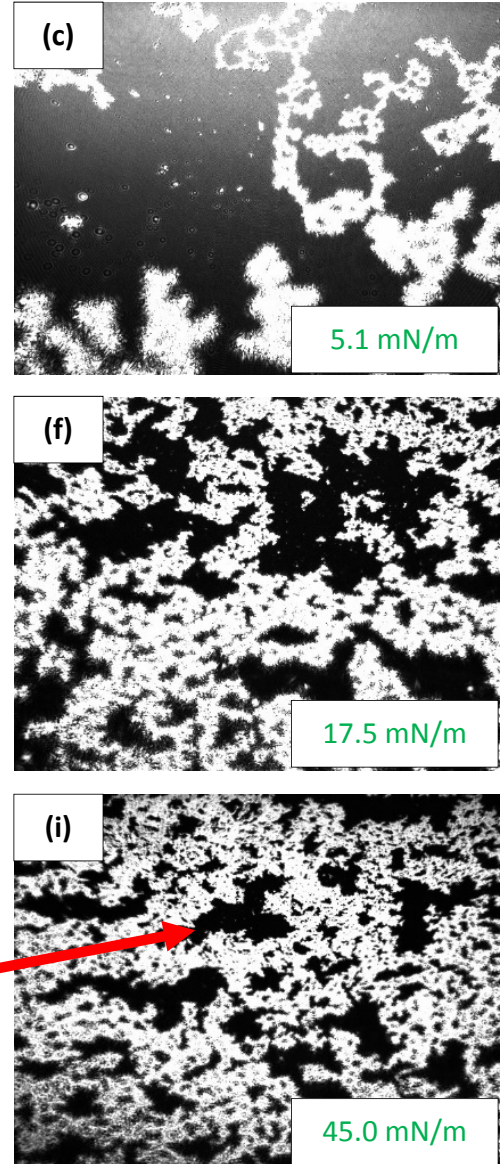

Figure 5.8: Brewster angle microscope images during compression of a Langmuir film of $\mathrm{MoS}_{2}$, showing a clean water surface (a) and the film at increasing pressures (b-i). Showing how surface coverage and film dynamics change upon compression. All images are $4 \mathrm{~mm}$ wide.

It can be seen that even at these low pressures, the flakes on the surface are already interacting with one another and seem to be forming domains that appear to be 'stringy' at the lowest pressures. Small spots representing small aggregates of material can also be seen on these images, which seem to have completely disappeared at high pressures, suggesting that the $\mathrm{MoS}_{2}$ film on the surface slowly builds up into large domains by the adhesion of these smaller regions. This also agrees with the hysteretic behaviour observed in fig 5.7. The images in fig $5.8(\mathrm{e}-\mathrm{i})$ demonstrate the film behaviour as the barriers approach their closed position, it was observed that the gaps in the film are gradually being reduced although even at the largest pressure there are still a number of visible gaps. To reach higher pressures and attempt to close these gaps, the BAM head must be removed so the dynamics here were not studied. 
An interesting observation is that in images (h) and (i), the film becomes tremendously static as highlighted by the shaped gap regions that are pointed out and have moved $<1 \mathrm{~mm}$. This is in stark contrast to the rest of the images where a completely different section of the film has been imaged. By observing the BAM images in real time, it can be seen that up to pressures of $\approx 20 \mathrm{mN} / \mathrm{m}$, there is still a lot of movement in the film which rapidly decreases as the pressure is raised above $25 \mathrm{mN} / \mathrm{m}$. This is somewhat in agreement with the pressure-area isotherm in fig 5.5 , which seems to show a change in gradient indicating a phase change from 'liquid' to 'solid' at approximately this pressure. The oscillating barrier experiment enabled the measurement of the film motion in reverse. The film was observed to become highly stable when the barriers were in their closed position with little movement, and as the barriers were re-opened the film held its stability until a critical pressure/area was reached when the domains of $\mathrm{MoS}_{2}$ become highly mobile and rapidly move from under the BAM head. It is proposed that the simple explanation for this is that due to the low area and high pressure of the film in these instances, the film is trapped due to the formation of a 'solid' phase, making it difficult for it to reorganise itself even if the surface is agitated. This could also explain why the gaps in the $\mathrm{MoS}_{2}$ regions do not seem to fully disappear at the highest measured pressures; especially since there is a large size distribution of flakes. On the other hand, as the barriers are re-opened, the $\mathrm{MoS}_{2}$ becomes free to move once this critical area is reached, perhaps helped by gentle agitation. This indicates the transition back to a 'liquid' or 'gas' phase, albeit with flakes that have aggregated into larger domains. The agitation suggested here comes from the movement of the motorised barriers themselves, as although this effect has been extremely dampened in the experimental setup, there are still small vibrations transferred to the trough which could provide enough energy for the film to display this behaviour. Furthermore, although held on a large marble slab, vibrations and other noise from the laboratory can agitate the water surface. Care also has to be taken to minimise air flows over the trough. The observation of a film at the air-water interface provides the first step for the deposition of an LB film, and demonstrates the effectiveness of electrospray for maintaining the material at the surface, due to the alleviation of mixing. 


\subsubsection{Deposition of a Langmuir-Blodgett Film}

The next stage of this work involved depositing the prepared Langmuir film on the surface of the water onto a nominal substrate, in this case $\mathrm{Si} / \mathrm{SiO}_{2}$ was used. In order to deposit a single layer via the Langmuir-Blodgett method, the substrate must be positioned through the air-water interface prior to cleaning and spreading of material, so that the substrate only needs to be 'pulled' through the interface once. Prior to the placement of an $\mathrm{SiO}_{2}$ substrate, it was cleaned with acetone and IPA for 5 minutes before drying with $\mathrm{N}_{2}$ and then ashed with oxygen plasma for 10 minutes. This gives the substrate a hydrophilic nature needed to make the $\operatorname{Mos}_{2}$, which is also hydrophilic, adhere ${ }^{37}$.
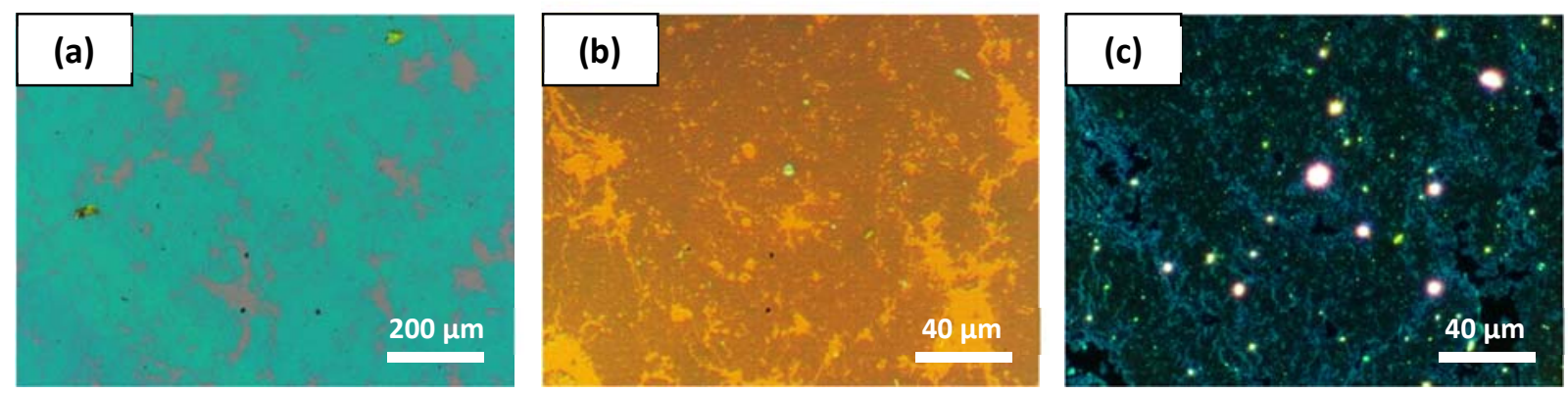

Figure 5.9: Optical images of a transferred $\mathrm{MoS}_{2}$ film under (a) 10x bright-field excitation, (b) 50x differential interference contrast excitation and (c) 50x dark-field excitation. The deposited film showed a similar morphology to that observed through the BAM, suggesting direct deposition onto the substrate. The colour of (a) suggests a film of $\sim 5-10$ layer thickness. (b) and (c) highlight thickness variations.

To transfer the film, $2.5 \mathrm{ml}$ of stock $\mathrm{MoS}_{2}$ solution was first sprayed onto the surface and compressed to $40 \mathrm{mN} / \mathrm{m}$ as in fig 5.4. Subsequently, the dipper raised the substrate through the subphase at a rate of $0.5 \mathrm{~mm} / \mathrm{min}$ whilst an active feedback system controlled the barrier positions to maintain a constant surface pressure. Due to the slow rate of the dipping arm, the substrate dries gradually in air as it is being pulled up, leading to a dry substrate by the end of the deposition. However, to remove any residue or adsorbed water left on the surface, the substrate was placed in a vacuum oven at $100^{\circ} \mathrm{C}$ for 4 hours. The optical images of the as-deposited film are shown in fig 5.9. It was instantly observed that the transferred film looks very similar to the images that were taken on the BAM in terms of its morphology; the optical images relate to a sixteenth of the BAM images. 
This provides strong evidence that the film on the water surface is being deposited to the substrate efficiently and that gaps in the deposited film are due to gaps already present on the water surface. Furthermore, an extremely high coverage is achieved following deposition. Through analysis of a number of areas within the film, the average coverage was found to be $89.9 \pm 2.7 \%$, indicating an extremely successful deposition via this process. The blue colour of the film compared to the $\mathrm{SiO}_{2}$ background in fig 5.9a also suggests that the film is much thicker than monolayer, with this colour normally indicating between 5-10 layers of $\mathrm{MoS}_{2}{ }^{38}$. The reason for this large thickness could be that during film formation and deposition of the $\mathrm{MoS}_{2}$, there is the possibility of aggregation occurring such that flakes are able to stack on top of one another. It could also be a result of the average layer thickness of the stock solution being on this order. Fig $5.9 \mathrm{~b}$ and $\mathrm{c}$ inspect the deposited film in more detail, whilst the DIC and dark-field modes allow thickness variations to be observed. It can be seen that the thickness variation across the film is relatively uniform with no obvious colour variation in fig $5.9 \mathrm{~b}$ and bright regions in fig 5.9c correlating well with the film-substrate boundaries. This observation suggests the latter explanation for the overall thickness of the film, as if there was significant aggregation and stacking taking place, such uniformity in the film thickness would not be expected. On the other hand, if the average flake thickness was between 5-10 layers, then this would explain the consistency of the deposited film. Finally, it should be noted that there was clear evidence of contaminants in the film, as highlighted by the bright spots in fig $5.9 \mathrm{c}$ which represent much larger features than the film itself, but these did not affect the deposition.

\subsubsection{Creating Optical Identification Devices}

Langmuir-Blodgett films of $\mathrm{MoS}_{2}$ were then deposited onto patterned substrates for isolation of individual devices to be used as unique identities. For deposition onto $\mathrm{SiO}_{2}$, a photoresist, Microposit ${ }^{\circledast}$ S1805 series ${ }^{39}$, was spin-coated onto the substrate at 4000rpm creating a layer approximately $0.5 \mu \mathrm{m}$ in thickness. The substrate was then baked at $115^{\circ} \mathrm{C}$ for 90 seconds before defining the device patterns through exposure to $365 \mathrm{~nm}$ light for 1 second using an optical mask and a mask aligner (Süss Microptec $\mathrm{MJB}^{40}{ }^{4}$ ) operating in hard contact mode. 
To remove the exposed resist, the sample was then soaked in a tetramethylammonium hydroxide (TMAH) based developer, MF-319 ${ }^{39}$, for 30 seconds before being rinsed with DI water and dried with $\mathrm{N}_{2}$. A Moorfield thermal evaporator was then used to deposit 5/100 $\mathrm{nm}$ of titanium and gold, respectively ${ }^{41}$. Finally, the unwanted resist, gold and titanium was removed using an N-methyl-2pyrrolidone (NMP)-based lift off chemical, remover $\mathrm{PG}^{39}$. The $\mathrm{MoS}_{2}$ was then deposited using the methods highlighted above yielding isolated devices for identification, shown in fig 5.10.
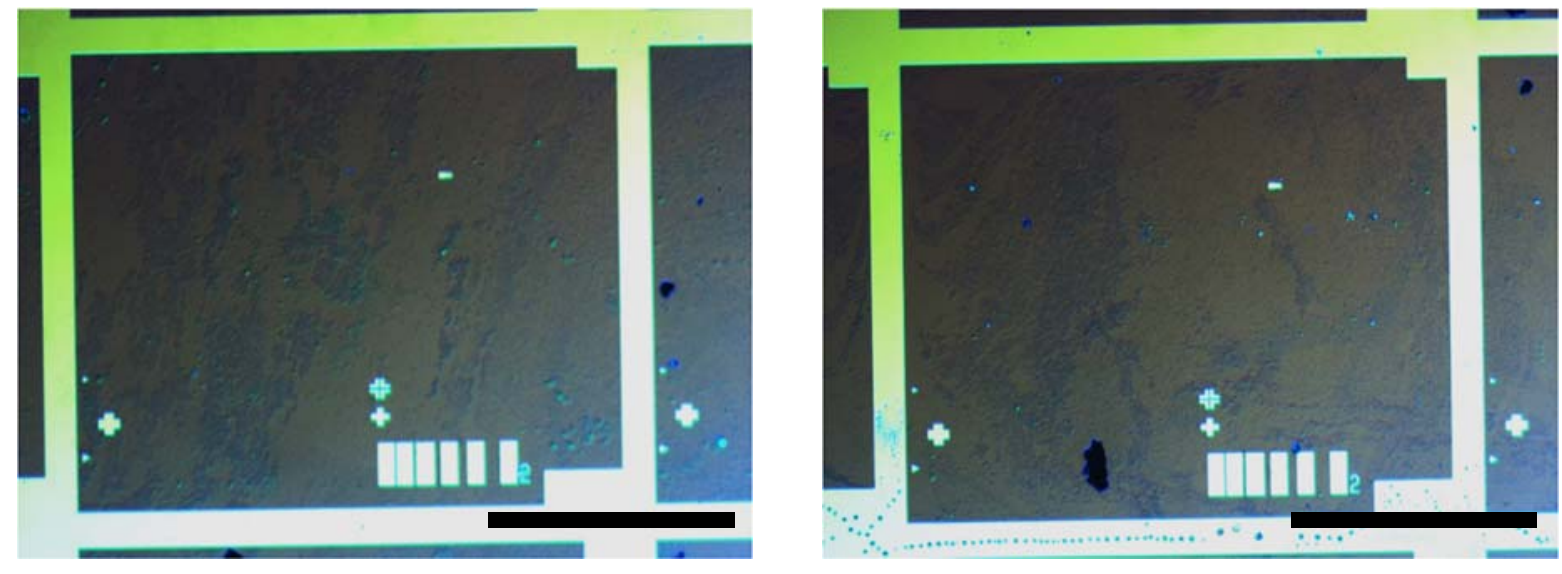

Figure 5.10: Optical images of $\mathrm{MoS}_{2}$ films deposited onto individual devices such that the variation in photoluminescence could be used for unique identification of the different tags, using 50x, DIC mode excitation. Scale bars are $750 \mu \mathrm{m}$.

It was found that the deposition was extremely efficient, even onto substrates that had undergone various fabrication treatments. Whilst the lower coverage area seen here is the result of using a lower pressure. However, the fabricated devices failed to show any photoluminescence (PL), with the mechanisms behind this being explored in the next section. This is of critical significance for unique identification, so determining whether the LB method is the limiting factor is extremely important.

\subsection{Analysis of Deposited Langmuir-Blodgett Films of $\mathrm{MoS}_{2}$}

\subsubsection{Quenched Photoluminescence of $\mathrm{MoS}_{2}$ Films}

To create devices that can be used for optical identification and use the quantum confinement of the flakes, it is a requirement that the film efficiently emits light through carrier recombination. As aforementioned, upon investigation of the deposited films with micro-photoluminescence, it was 
observed that the emission had completely quenched in all areas, even when using high laser power densities up to $20 \mathrm{~mW} / \mu \mathrm{m}^{2}$.

There are many reasons that this could occur, firstly $\mathrm{MoS}_{2}$ is intrinsically $\mathrm{n}$-doped and it has been shown that interactions with the $\mathrm{SiO}_{2}$ substrate can further $\mathrm{n}$-dope the $\mathrm{MoS}_{2}$, leading to a reduction in the emission intensity, as opposed to freestanding $\mathrm{MoS}_{2}{ }^{42}$. This effect is thought to be amplified in the case of LB films as it is anticipated that adsorbed water molecules remain adhered to the $\mathrm{MoS}_{2}$ even after baking. These bond to the surface and can create a further excess of electrons which could explain the complete quenching of emission. To investigate this further, the $\mathrm{MoS}_{2}$ solution was also drop-cast onto an identical $\mathrm{SiO}_{2}$ substrate whilst on a hot-plate at $100^{\circ} \mathrm{C}$. This allowed rapid removal of the ethanol/water solute leaving a much smaller percentage of adsorbed molecules behind. However, upon inspection, the drop-cast film displayed no PL emission, even though the density of flakes via this method is substantial, implying that this is not the main driving mechanism behind the PL quenching of films. There is also the possibility that the $\mathrm{MoS}_{2}$ in solution is in the $1 \mathrm{~T}$, metallic phase, which displays quenched photoluminescence ${ }^{43}$. However, through annealing in $\mathrm{N}_{2}$ at $400^{\circ} \mathrm{C}$ the majority of the $2 \mathrm{H}$, semiconducting phase can be returned ${ }^{44}$. This method was tested for the deposited films and it was found that there was still no emission, which provides no evidence for which phase the $\mathrm{MoS}_{2}$ solution is in, but allows this quenching mechanism to be disregarded. Other reasons for the lack of emission are not necessarily attributed to quenching, but could prevent emission from the film in the first place. In particular, these are bottlenecks arising from size dependent effects of the flakes in the stock solution. For example, if the monolayer content contained within the solution was negligible then observation of PL would be difficult. Moreover, the lateral sizes of flakes are also important as if they are below a certain limit ( $28 \mathrm{~nm} \times 8 \mathrm{~nm}$ from [26]), edge states can quench the luminescence from a given flake due to edge-activated trapping of excitons. To investigate these size effects, a number of methods were employed, with the results shown in the following section. 


\subsubsection{Investigation of Possible Quenching Mechanisms}

Firstly, the deposited films were inspected with a scanning electron microscope (SEM), as this allows the contents of the film to be probed on a number of different scales whilst allowing an idea of the thickness of the film to be estimated.
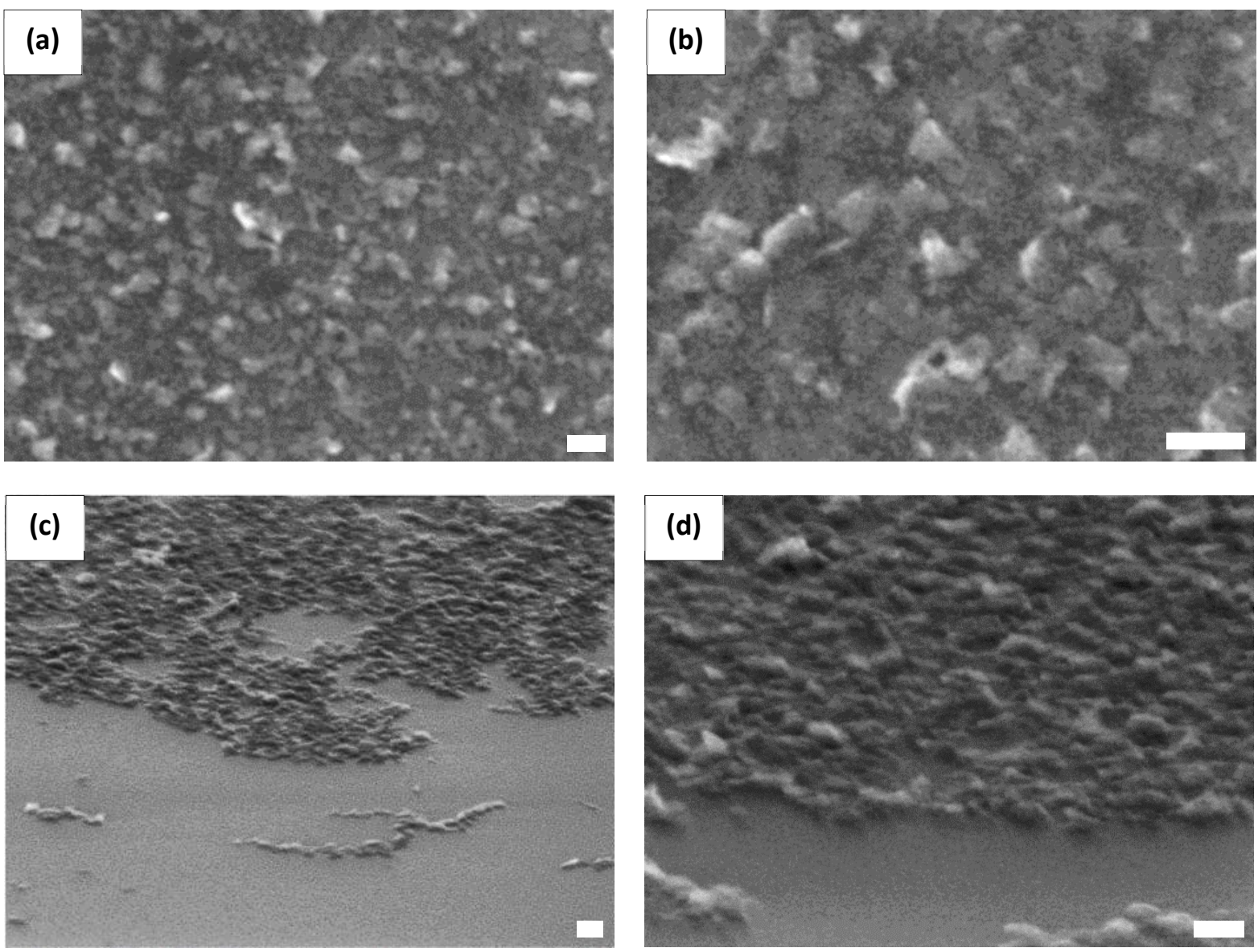

Figure 5.11: Scanning electron microscopy images of a deposited $\mathrm{MoS}_{2}$ film, (a) and (b) are from above and highlight the size and distribution of flakes, (c) and (d) are from an angle of $70^{\circ}$ with respect to the normal which indicate the thickness uniformity of the deposited film. All scale bars represent $100 \mathrm{~nm}$ whilst the acceleration voltage was set to $15 \mathrm{kV}$, due to high resolution requirements.

The SEM images of the film deposited in fig 5.9 are shown in fig 5.11. It was observed that on the areas of the substrate where the film has deposited, the film appears to be uniform with a constant coverage of flakes across these areas. However, the brightness of the flakes indicates that a large proportion are not monolayer, as SEM measurements usually yields a dark region with respect to the substrate when imaging monolayers ${ }^{45}$. 
This data also allows an estimate for the average lateral flake size, although determining this is difficult due to the shape variation of the flakes. What can be deduced is that the estimate of $100 \mathrm{~nm} \times 100$ $\mathrm{nm}$ in the beginning of the chapter was clearly an over-estimate. Through examination of a number of SEM images, the average flake size actually appears to be in the region of $40-60 \mathrm{~nm}$ and is explored in detail in the AFM measurements later.

The images in fig 5.11c and d were taken with the sample angled at $70^{\circ}$. The deposition seems to have resulted in a relatively consistent height across the film with no large areas of differing heights being visible, suggesting that one layer has deposited during dipping and no aggregation has occurred. It was observed that the flakes, however, are not flat to the substrate and are arranged in a seemingly random orientation. This kind of behaviour is not unexpected as the $\mathrm{MoS}_{2}$ is hydrophilic so does not have a tendency to order itself perfectly on the water surface as in the conventional films used in the LB technique. Thus, when this film is deposited, there is nothing to prevent the individual flakes from overlapping and forming a disordered film. This is not necessarily a disadvantage, as it could minimise interaction with the substrate resulting in less $n$-doping of the $\operatorname{MoS}_{2}$. On the other hand, this will limit the adhesion to the substrate which could result in films that can be removed easily, in stark contrast to mechanically exfoliated monolayers which are extremely well adhered to the underlying substrate.
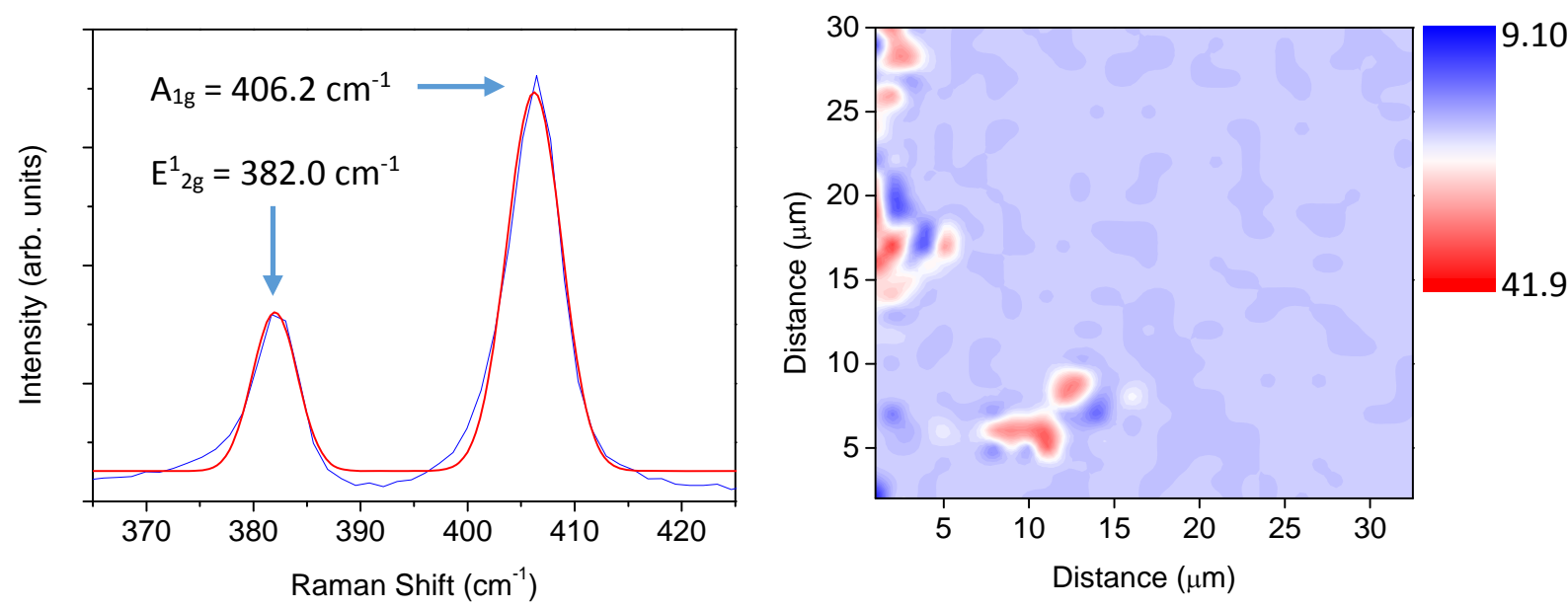

Figure 5.12: Raman spectroscopy results from a deposited $\mathrm{MoS}_{2}$ film, (a) measurement of a single position in the film, measured data (blue) and Gaussian fitted spectrum (red), (b) map of the peak spacing between $A_{1 g}$ and $E^{1}{ }_{2 g}$. 
To further investigate the population of monolayers, Raman spectroscopy was performed on a number of areas in the film, with representative data shown in fig 5.12. In $\mathrm{MoS}_{2}$, two peaks known as the $A_{1 g}$ and $E^{1}{ }_{2 g}$ peaks arise in the Raman spectra due to in-plane and out-of-plane oscillations, respectively. These peaks are often used as an indicator for layer thickness ${ }^{46}$. The intensities and linewidths provide some information about the number of layers, but the key metric used is the spacing between these peaks, which reduces to approximately $18-19 \mathrm{~cm}^{-1}$ for monolayer flakes.

Fig 5.12a displays a typical measurement from a part of the $\mathrm{MoS}_{2}$ film (blue trace) as well as the fitted Gaussian curves used to extract the peak centres for fig 5.12b. In the data plotted in fig 5.12b, the average peak spacing was plotted against position in the film, it was found that the mean value in the film is $24.65 \pm 0.08 \mathrm{~cm}^{-1}$, where 0.08 is the standard error calculated from the spread in results. This represents a value similar to what is observed in bulk $\mathrm{MoS}_{2}$ (the difference converges to the bulk value above $\approx 5$ layers). This further confides that the monolayer content of the film is negligible, meaning it will be difficult to observe light emission. The data in fig $5.12 \mathrm{~b}$ does display regions that drop to approximately $15-20 \mathrm{~cm}^{-1}$, however, upon inspection of these traces it was found that they were taken from the edges of the film and led to spectra with a very low signal to noise ratio. Thus the Gaussian fitting of these spectra had much less precision and in some cases fitted the wrong peak altogether, so these points were ignored as anomalous.

To probe the actual height of the flakes in the film, atomic force microscopy (AFM) data was collected with the contact-mode method of operation. Here, the scan rate was set to $1.0 \mathrm{~Hz}$, sampling 256 points per line, with an amplitude set-point and drive amplitude of $357.8 \mathrm{mV}$ and $100.0 \mathrm{mV}$, respectively. Fig 5.13a shows a typically acquired image from the film surface, before capture an area that showed a gap in the film was found such that there is always a flat substrate to enable accurate height analysis. The height profiles in fig $5.13 \mathrm{c}$ represent cross sections of the lines drawn on the topography image. What is first noticeable is the large range in apparent heights of flakes, with a minima and maxima of $2.3 \pm 0.2 \mathrm{~nm}$ and $20.4 \pm 0.2 \mathrm{~nm}$, respectively. These heights correspond to thicknesses of $\sim 4$ to 32 layers. These are only approximations as it is difficult to obtain an exact measurement of the height 
of flakes in the film on this scale, especially since it is expected that there will be adsorbed molecules on the flakes that will affect the results. The average height was calculated through examination of 50 different flakes from 5 different regions of the film and found to be $7.6 \pm 0.6 \mathrm{~nm}$. This corresponds to an average number of layers of $\sim 12$, calculated using an apparent height of monolayer $\mathrm{MoS}_{2}$ of $6.15 \AA^{47}$.
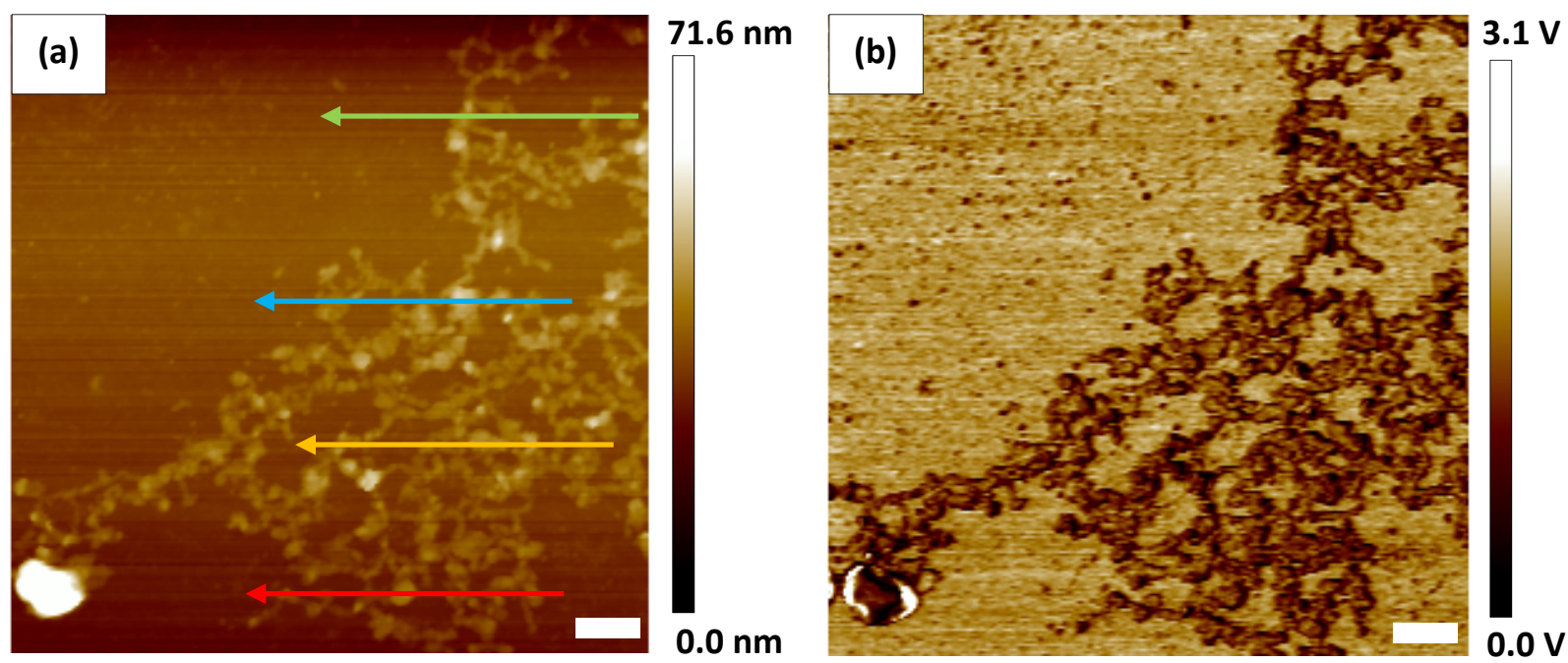

(c)
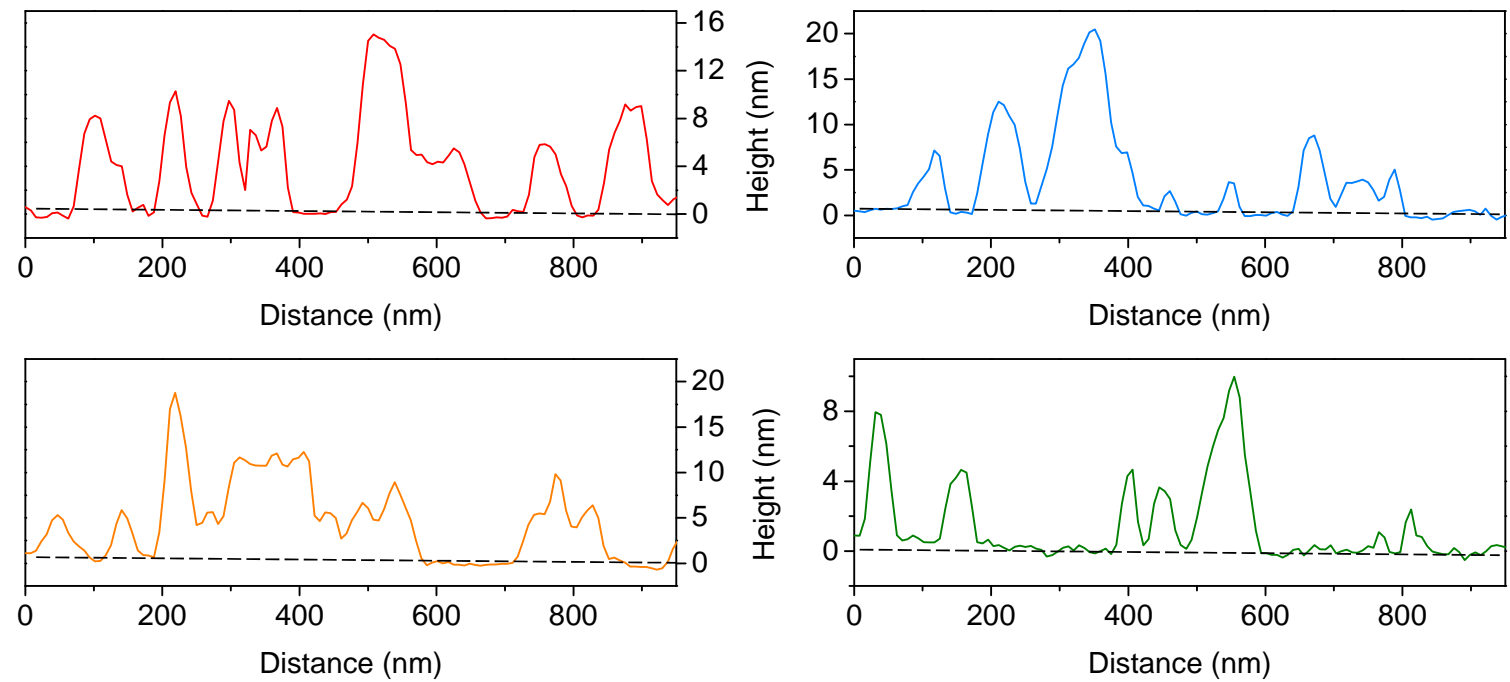

Figure 5.13: AFM analysis of a deposited $\mathrm{MoS}_{2}$ film showing (a) a typical topographic image of an area of the film, (b) UFM image of the same area, (c) 4 height profiles from the sections highlighted in (a). Scale bars represent $200 \mathrm{~nm}$.

What can also be inferred from fig $5.13 \mathrm{c}$ is the lateral size of the flakes. As the flakes show no preferred direction, a cross-section in the $x$-direction as in fig 5.13a was used for length analysis. Through analysis of 50 different flakes found within the film, the average lateral sizes of the flakes was 
calculated to be $55.7 \pm 7.4 \mathrm{~nm}$, consistent with the SEM results. Both of the previous errors are standard errors taken from the spread of results. It should be noted that as the flakes are thought not to preferentially orientate themselves parallel to the substrate, the measurements of thickness and length are assumed to be on flakes that are perfectly aligned with the substrate. The measurement of the size distribution of the flakes indicates that it is likely not edge-effects that is quenching the emission and it has been concluded that it is principally due to a lack of monolayers in the film. Although the adsorption, substrate and phase are still expected to play a small role in affecting the flake properties.

Fig $5.13 \mathrm{~b}$ shows a UFM measurement of the same film area shown in (a), where the substrate is oscillated at a frequency of 4-5 MHz, with an amplitude of 5-10 $\AA$. The key advantage of UFM is that it allows the investigation of stiffness variations at the substrate-flake interface. In the UFM image, regions of brighter contrast correspond to greater mechanical stiffness, whereas totally black regions indicate complete decoupling from the substrate. Thus, the adhesion of the film to the substrate can be analysed to give some indication to how the film interacts with the water. It can be seen from the figure that, as expected, the silicon substrate shows a high stiffness whilst being extremely uniform across the surface. On the other hand, in places where the film is adhered, there is a contrast decrease of up to $66 \%$ and a large variation in contrasts across the film, with a decrease of $24.4 \pm 1.8 \%$, on average. This variation could be due to the lack of orientation, or stacking which would result in poor and non-uniform adhesion to the substrate compared to mechanically exfoliated $\mathrm{MoS}_{2}$, which shows a much brighter contrast whilst being uniform over the entire flake area ${ }^{48}$.

Through observation of the UFM data, it became clear that this would be a useful method to find monolayers on the sample due to the enhanced contrast difference between the film and substrate. After careful exploration, individual monolayers were identified and their AFM and UFM data was taken, with a typical measurement plotted in fig 5.14. It can be seen in the height image's profile that the height of the monolayer is greater than expected (6.15 $)$ ), it also shows a 'bubble-like' profile as 
(a)

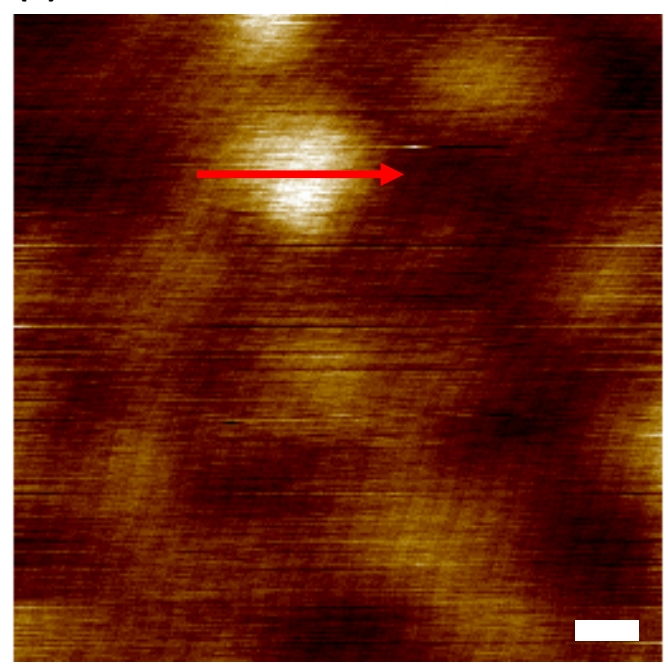

(c)

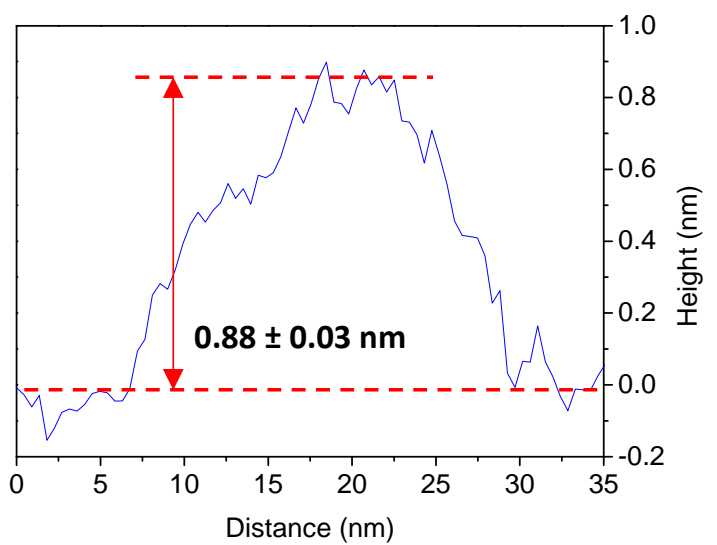

(b)
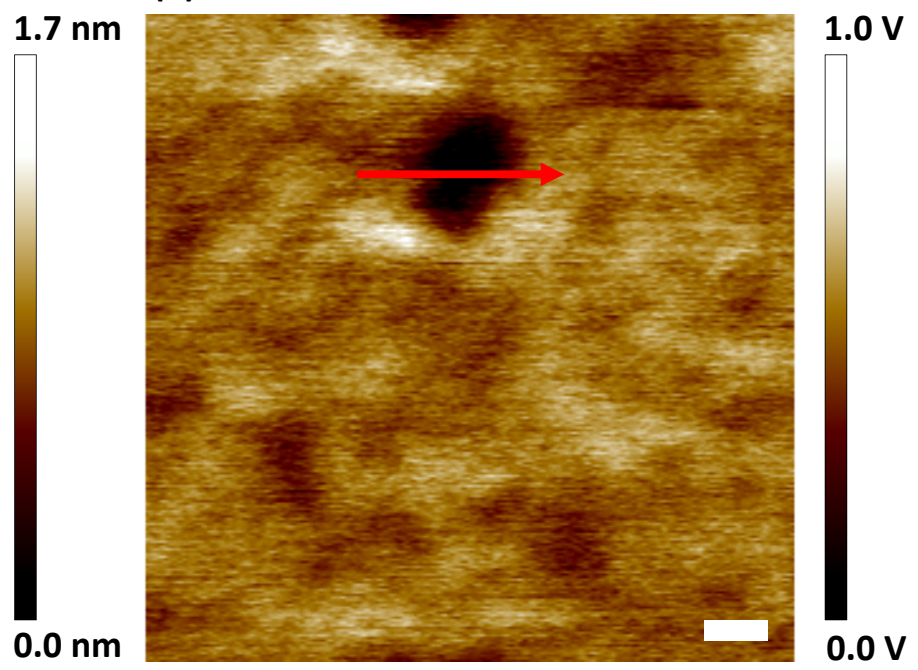

(d)

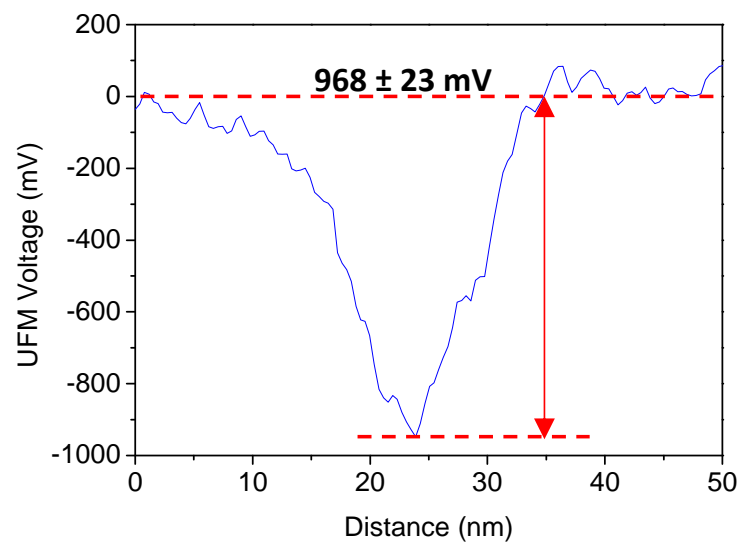

Figure 5.14: AFM/UFM analysis of individual monolayers, (a) topography image, (b) UFM image, (c) height profile from (a), (d) voltage profile from (b). This demonstrates the deviation in thickness from an isolated monolayer due to the presence of adsorbed species, whilst indicating that the monolayers are strongly decoupled from the substrate. Scale bars are $10 \mathrm{~nm}$.

opposed to a clear step that is conventionally seen in AFM of $\mathrm{MoS}_{2}$. The gain in height could possibly be explained through adsorption of water and other molecules whilst in the pristine solution or at the air-water interface; if there are molecules adsorbed to the monolayer then the actual height of a $\mathrm{MoS}_{2}$ monolayer will appear larger. The height of a water molecule is $2.75 \AA$, if it is assumed that the monolayer contains a single molecular layer of water underneath it, and the dangling bonds at the edge of the monolayer are adhered to the substrate, then this would give the monolayer a 'bubblelike' shape. Adjusting the value for a monolayer of $\mathrm{MoS}_{2}$ using this value gives an apparent height of $8.90 \AA$ A. This agrees extremely well with the height of the monolayer flakes that were found, a 
representative monolayer is shown in fig $5.14 \mathrm{a}$ and has a height of $0.88 \pm 0.03 \mathrm{~nm}$; the error comes from the uncertainty in measuring the baselines for top and bottom of the flake. Using this value to re-calculate the average number of layers yields a value of $\approx 8$, consistent with the blue colour observed in fig 5.9 (although this colour will now be affected through interaction with the adsorbed water). The profile of the UFM image shown in fig $5.14 \mathrm{~d}$ demonstrates the lack of adhesion of monolayers to the substrate.

As opposed to the large signal seen from the silicon substrate, the monolayer shows a reduction in contrast of $96.8 \pm 2.3 \%$, indicating that the monolayer is effectively completely decoupled from the substrate. Complimenting this observation with the monolayer's 'bubble-like' profile suggests that the adsorbed water has likely been trapped underneath the monolayer during deposition. This would result in a 'soft' measurement on the UFM as the water is compressible, whilst producing a dome profile due to the inability for the monolayer to adhere to the substrate other than at the edges. The presence of adsorbed water is likely uniform across the substrate, thus, adsorbed water could also play a key role in quenching the PL intensity.

In summary, the SEM, Raman spectroscopy, AFM and UFM data all seem to suggest that the photoluminescence is not present in transferred films due to primarily a low monolayer content. Although there is evidence for adsorbed molecules of water trapped beneath the film, which would n-dope the $\mathrm{MoS}_{2}$, quenching the PL further. Although this is a setback for this specific application, multi-layer films of $\mathrm{MoS}_{2}$ can be used for other purposes, such as field effect transistors ${ }^{49}$ and phototransistors ${ }^{50}$. Furthermore, by taking advantage of the large family of $2 \mathrm{D}$ materials, a material that emits when in its multi-layer form, such as InSe, could be applied for this application ${ }^{51}$. Furthermore, the values for the average measured layer thickness and lateral size of individual flakes can then be used to re-calculate the required volume of solution for a 'solid' phase to form on the water surface. This calculation yields a value for the starting volume of $\sim 5 \mathrm{ml}$. Thus, the observation of no critical pressure in fig 5.5 is expected and in future higher starting volumes should be used. 


\subsubsection{A Different Source of $\mathrm{MoS}_{2}$}

To solve the problem of unmeasurable photoluminescence of the measured LB films. A new source of $\mathrm{MoS}_{2}$ was used. The details of this solution are reported in ref. [26] and comprises $\mathrm{MoS}_{2}$ flakes encapsulated in sodium cholate (a surfactant) suspended in an aqueous solution. Preliminary results have been captured and these are shown in fig 5.15. As the $\mathrm{MoS}_{2}$ was suspended in aqueous solution, the electrospray system had to be slightly altered. This mainly consisted of changing the inner diameter of the needle to $150 \mu \mathrm{m}$ to increase the flow rate of the solution. The flow rate slows down considerably when using the same needle as before as the surface tension of the solution is much larger due to a lack of ethanol, hence larger forces act to keep the solution in the syringe. The pressurearea isotherm is shown in (a), which displays similar characteristics to the isotherms seen for the previous $\mathrm{MoS}_{2}$ solution. The pressure rise was significantly high considering $0.5 \mathrm{ml}$ of solution with only $1 \mathrm{~g} / \mathrm{L}$ concentration was deposited.
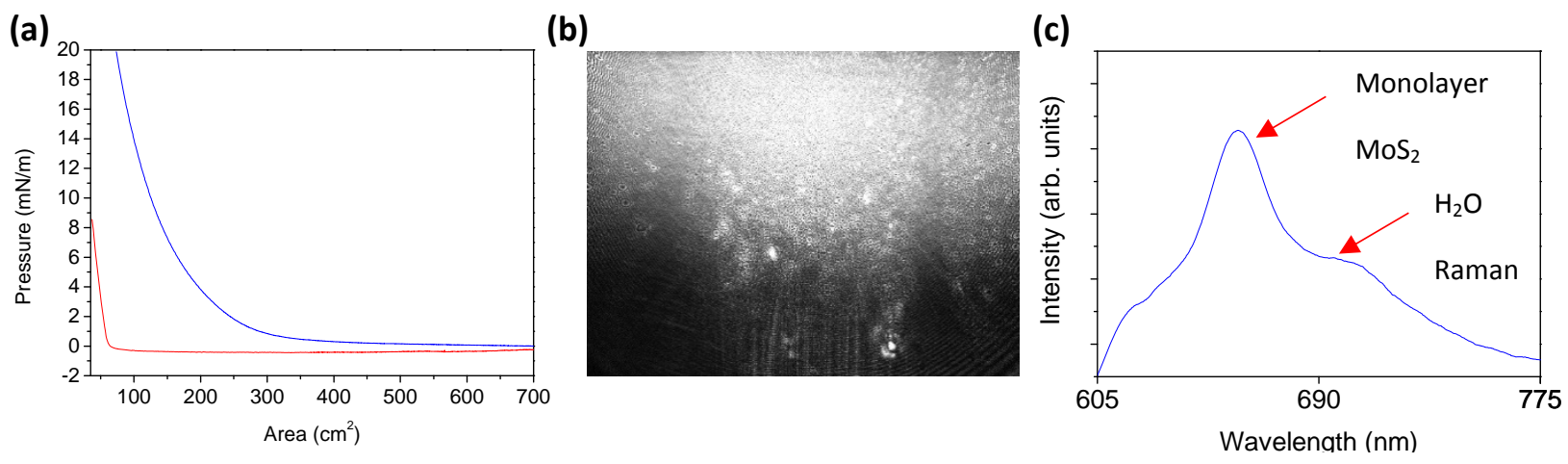

Figure 5.15: Results of using a 75\% monolayer content solution for Langmuir-Blodgett deposition, with (a) Typical pressure-area isotherm, (b) BAM image and (c) Photoluminescence signal.

The BAM image shows a considerably different morphology on the water surface, it can be seen that the contrast is largely reduced with respect to the data in fig 5.8 , suggesting that a much thinner film is forming on the surface; as expected from the large monolayer content of the dispersion. Furthermore, the addition of surfactants to the flakes will affect the surface chemistry, changing the interaction mechanisms between the flakes and seems to result in a film with higher uniformity on the water surface. The micro-photoluminescence data from a deposited Langmuir-Blodgett film is shown in c, a peak corresponding to the neutral exciton in $\mathrm{MoS}_{2}$ is observed at $660 \mathrm{~nm}$, overlapping 
with the weak, broad Raman signal of water at $\approx 650 \mathrm{~nm}^{52}$. This signal was observed at all places on the deposited film. Interestingly, the signal does not appear instantly but rather the broad water Raman peak is observed with a much greater intensity. After a few seconds, the Raman signal reduces, allowing the emission from the monolayer to be observed. This suggests that the laser impinging the sample is locally heating the substrate causing the adsorbed water to be ablated. The remaining signal observed suggests that some water is still not removed, due to 'trapping' indicated by the UFM experiments. Although emission has been observed, it is extremely weak and approximately 2 orders of magnitude less in intensity than a drop cast sample. A number of methods can be employed to increase this intensity. Firstly, changing the subphase to something other than water that will not quench the emission can be explored. LB deposition also allows for the choice of an arbitrary substrate, thus the use of different substrates such as gold and mica can enhance $\mathrm{PL}^{53}$. Post-treatment of the $\mathrm{MoS}_{2}$, with superacid, for example, can also be performed to increase the quantum yield following deposition ${ }^{54}$.

\subsection{Summary}

In this work, it was suggested that the atomic imperfections present in 2D materials could be used for unique identification. These imperfections alter the physical properties of the material and should be measurable via macroscopic methods, as verified using photoluminescence on individual flakes. However, individual flakes are limited in their application potential, thus it was hypothesized that the Langmuir-Blodgett technique could be used to create scalable large-area films of $\mathrm{MoS}_{2}$ to create macroscopic identification tags. Furthermore, the creation of such thin films of $2 \mathrm{D}$ materials is of interest for a range of other applications, such as field effect transistors.

This prediction was tested by employing an electrospray technique, which showed the successful deposition of large-area films of $\mathrm{MoS}_{2}$ for the first time. These $\mathrm{MoS}_{2}$ solutions were deposited onto a water surface and the presence of a Langmuir film was confirmed by pressure-area isotherms and Brewster angle microscopy. These surface films remained on the surface under a number of 
compression and expansion cycles, whilst displaying hysteresis effects suggesting an elastic nature for the film. These films were then transferred onto silicon substrates containing photolithographydefined patterns for individual devices to allow unique characterisation. The transferred films displayed no photoluminescence so a number of experiments were performed to elucidate the cause of this quenching. Raman spectroscopy and scanning electron, atomic force and ultrasonic force microscopy was performed on the deposited films to understand these effects. The SEM, AFM and Raman spectroscopy results showed uniform film deposition and it was concluded that the principal reason for no emission was a very low monolayer content. The average thickness of flakes within the deposited films was found to be much larger than monolayer, measuring $7.6 \pm 0.6 \mathrm{~nm}$ and representing $\approx 12$ layers. The UFM data showed that identified monolayers displayed a bubble-like profile whilst displaying a $96.8 \pm 2.3 \%$ reduction in contrast in the flake centre which indicates almost complete delamination from the substrate. This indicated the presence of water underneath the flake and allowed for the correction of the measured monolayer height, altering the average number of layers to $\approx 8$ layers, in agreeance with the optical microscopy results.

Finally, a different starting dispersion was employed that held a monolayer content of $75 \%$ in aqueous solution. Preliminary results have shown that these too can be successfully deposited via the LB technique, although the underlying interaction mechanisms seem to be different. These deposited films showed reproducible photoluminescence from all areas.

It has been shown that it is now possible to create large-area thin films of $\mathrm{MoS}_{2}$ and this process can now be tuned to create identity tags with ideal properties. Future work will focus upon increasing the intensity of deposited films. This can be achieved through the alteration of the subphase or the substrate. Furthermore, processed samples can be treated to enhance the quantum yield from flakes. The exploration of the deposition of a range of other 2D materials via this technique will be investigated; InSe is a promising candidate.. Different 2D materials will also be deposited sequentially to create heterostructures that should give a larger emission bandwidth. 


\section{Bibliography}

${ }^{1}$ K. Novoselov et al., "Electric Field Effect in Atomically Thin Carbon Films", Science, 306, 5696 (2004).

${ }^{2}$ K. I. Bolotin et al., "Ultrahigh Electron Mobility in Suspended Graphene”, Solid State Communications, 146, 9 (2008).

3 J. Lee et al., "Estimation of Young's Modulus of Graphene by Raman Spectroscopy", Nano Lett., 12, 9 (2012).

${ }^{4}$ M. Sprickle et al., "First Direct Observation of a Nearly Ideal Graphene Band Structure", Phys. Rev. Lett., 103, 226803 (2009).

${ }^{5}$ Z. Chen et al., "Graphene Nano-ribbon Electronics", Physica E: Low-dimensional Systems and Nanostructures, 40, 2 (2007).

${ }^{6}$ M. Bacon et al., "Graphene Quantum Dots”, Particle \& Particles Systems Characterization, 31, 4 (2013).

${ }^{7}$ K. F. Mak et al., “Atomically Thin $\mathrm{MoS}_{2}$ : A New Direct-gap Semiconductor”, Phys. Rev. Lett., 105, 136805 (2010).

${ }^{8}$ H. R. Gutiérrez et al., "Extraordinary Room-temperature Photoluminescence in Triangular WS 2 Monolayers", Nano. Lett., 13, 8 (2013).

9 J. Kang et al., "A Unified Understanding of the Thickness-dependent Bandgap Transition in Hexagonal Twodimensional Semiconductors", J. Phys. Chem., 7, 597 (2016).

10 Poljak et al., "Influence of Edge Defects, Vacancies, and Potential Fluctuations on Transport Properties of Extremely Scaled Graphene Nanoribbons", IEEE Transactions on Electron Devices, 59, 12 (2012).

${ }^{11}$ D. Usachov et al., "The Chemistry of Imperfections in N-Graphene", Nano. Lett., 14, 9 (2014).

12 X. Ji et al., "Infleunce of Edge Imperfections on the Transport Behaviour of Graphene Nanomeshes", Nanoscale, 5, 2527 (2013).

${ }^{13}$ A. Fasolino et al., "Intrinsic Ripples in Graphene”, Nat. Mater., 6, 858 (2007).

${ }^{14}$ M. Koperrski et al., "Single Photon Emitters inExfoliated WSe ${ }_{2}$ Structures", Nat. Nano., 10, 503 (2015).

${ }^{15}$ A. Castellanos-Gomez, "Why All the Fuss About Semiconductors?", Nat. Photon., 10, 202 (2016).

${ }^{16}$ P. Blake et al., “Making Graphene Visible”, Appl. Phys. Lett., 91, 063124 (2007).

${ }^{17}$ P. Tonndorf et al., "Photoluminescence Emission and Raman Response of Monolayer $\mathrm{MoS}_{2}, \mathrm{MoSe}_{2}$ and WSe", Optics Express, 21, 4 (2013).

18 S. Tongay et al., "Defects Activated Photoluminescence in Two-dimensional Semiconductors: Interplay Between Bound, Charged and Free Excitons", Sci. Rep., 3, 2657 (2013).

${ }^{19}$ X. H. Wang et al., "Photoluminescence and Raman Mapping Characterisation of WS 2 Monolayers Prepared Using Top-down and Bottom-up Methods", J. Mater. Chem. C, 3, 2589 (2015).

${ }^{20}$ K. Wei et al., "Large Range Modification of Exciton Species in Monolayer WS," Appl. Optics, 55, 23 (2016).

${ }^{21}$ M. S. Kim et al., "Photoluminescence Wavelength Variation of Monolayer $\mathrm{MoS}_{2}$ by Oxygen Plasma Treatment", Thin Solid Films, 590, 318 (2015).

${ }^{22}$ H. F. Lie et al., "CVD Growth of $\mathrm{MoS}_{2}$-based Two-dimensional Materials", Chem. Vap. Deposition, 21, 241 (2015). 
${ }^{23} \mathrm{~K}$. Kang et al., "High-mobility Three-atom-thick Semiconducting Films with Wafer-scale Homogeneity", Nature, 520, 656 (2015).

${ }^{24}$ Y. Gong et al., "Vertical and In-plane Heterostructures from WS $2 / M_{2} S_{2}$ Monolayers", Nat. Mater., 13, 1135 (2014).

${ }^{25}$ V. Nicolosi et al., "Liquid Exfoliation of Layered Materials", Science, 340, 1226419 (2013).

${ }^{26}$ C. Backes et al., "Production of Highly Monolayer Enriched Dispersions of Liquid-exfoliated Nanosheets by Liquid Cascade Centrifugation", ACS Nano, 10, 1589 (2016).

${ }^{27}$ F. Bonaccorso et al., "Production and Processing of Graphene and 2D Crystals", Materials Today, 15, 12 (2012).

${ }^{28}$ L. J. Cote et al., "Langmuir-Blodgett Assembly of Graphite Oxide Single Layers", J. Am. Chem. Soc., 131, 1043 (2009).

${ }^{29}$ X. Li et al., "Highly Conducting Graphene Sheets and Langmuir-Blodgett Films”, Nat. Nanotech., 3, 538 (2008).

${ }^{30} \mathrm{H}$. Tachibana et al., "Highly Conductive Inorganic-organic Hybrid Langmuir-Blodgett Films Based on $\mathrm{MoS}_{2}$ ", Chem. Mater., 12, 854 (2000).

${ }^{31} \mathrm{Y}$. Taguchi et al., "Fabrication of Hybrid Layered Films of $\mathrm{MoS}_{2}$ and an Amphiphilic Ammonium Cation Using the Langmuir-Blodgett Technique", Langmuir, 14, 6550 (1998).

32 W. M. R. Divigalpitiya et al., "Spread Films of Single Molecular Transition-metal Sulfides", Appl. Surface Sci., 48, 572 (1990).

${ }^{33} \mathrm{H}$. Nie et al., "High-yield Spreading of Water-miscible Solvents on Water for Langmuir-Blodgett Assembly", J. Am. Chem. Soc., 137, 10683 (2015).

${ }^{34}$ www.graphene-supermarket.com

${ }^{35}$ S. S. Grønborg et al., "Synthesis of Epitaxial Single-layer MoS2 on Au(111)", Langmuir, 31, 9700 (2015).

${ }^{36}$ www.nordson.com

${ }^{37}$ P. K. Chow et al., "Wetting of Mono and Few-layered $\mathrm{WS}_{2}$ and $\mathrm{MoS}_{2}$ Films Supported on $\mathrm{Si} / \mathrm{SiO}_{2}$ Substrates", ACS Nano, 9, 3 (2015).

${ }^{38} \mathrm{H}$. Li et al., "Rapid and Reliable Thickness Identification of Two-dimensional Nanosheets Using Optical Microscopy", ACS Nano, 7, 11 (2013).

${ }^{39}$ www.microchem.com

${ }^{40}$ www.suss.com

${ }^{41}$ www.moorfield.co.uk

${ }^{42}$ N. Scheuschner et al., "Photoluminescence of Freestanding Single- and Few-layer MoS2", Phys. Rev. B, 89, 125406 (2014).

${ }^{43}$ R. Kappera et al., "Phase-engineered Low-resistance Contacts for Ultrathin $\mathrm{MoS}_{2}$ Transistors", Nat. Mater., 13, 1128 (2014).

${ }^{44}$ G. Eda et al., "Photoluminescence from Chemically Exfoliated MoS2", Nano Lett., 11, 12 (2011). 
${ }^{45}$ Y. Lee et al., "Characterization of the Structural Defects in CVD-grown Monolayered MoS 2 Using Near-field Photoluminescence Imaging", Nanoscale, 7, 11909 (2015).

${ }^{46}$ C. Lee et al., "Anomalous Lattice Vibrations of Single- and Few-layered MoS2”, ACS Nano, 4, 5 (2010).

${ }^{47}$ Y. Saito et al., "Superconductivity Protected by Spin-Valley Locking in lon-gated MoS S", Nat. Phys., 12, 144 (2016).

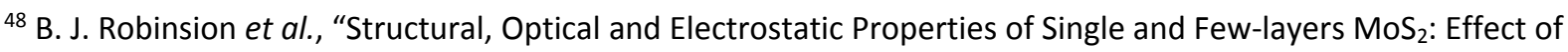
Substrate", 2D Mater., 2, 015005 (2015).

${ }^{49}$ N. R. Pradhan et al., "Intrinsic Carrier Mobility of Multi-layered $\mathrm{MoS}_{2}$ Field-effect Transistors on $\mathrm{SiO}_{2}$ ", Appl. Phys. Lett., 102, 123105 (2013).

${ }^{50}$ H. S. Lee et al., "MoS 2 Nanosheet Phototransistors with Thickness-modulated Optical Energy Gap", Nano. Lett., 12, 7 (2012).

${ }^{51}$ G. W. Mudd et al., "Tuning the Bandgap of Exfoliated InSe Nanosheets by Quantum Confinement", Adv. Mater., 25, 40(2013).

52 S. M. Baschenko \& L. S. Marchenko, "On Raman Spectra of Water, its Structure and Dependence on Temperature", Semiconductor Physics, Quantum Electronics and Optoelectronics, 14, 1 (2011).

${ }^{53}$ M. Buscema et al., "The Effect of the Substrate on the Raman and Photoluminescence Emission of Single-layer $\mathrm{MoS}_{2}$ ", Nano Research, 7, 4 (2014).

${ }^{54}$ M. Amani et al., "Near-unity Photoluminescence Quantum Yield in MoS$_{2}$ ”, Science, 350, 6264 (2015). 


\section{Chapter 6}

\section{Building Optoelectronic Heterostructures with Langmuir-Blodgett Deposition}

\subsection{Introduction}

The flexibility of the Langmuir-Blodgett technique for the deposition of unconventional materials has already been highlighted in the previous chapter. This ability, coupled with the ease of depositing such materials onto a range of substrates and its long-range order, results in the outstanding ability to design and fabricate novel heterostructures for optoelectronic device applications. In this chapter, the use of this method for the fabrication of complex heterostructures comprising of semiconducting colloidal quantum dots (CQDs) sandwiched between graphene sheets, is shown for the first time. This allows the scalable creation of hybrid quantum systems that utilise both two-dimensional and zerodimensional confinement. CQDs provide excellent light emitting and absorbing properties, whilst allowing flexible tuning of their band gap through control of their dimensions. Graphene, on the other hand, is an excellent transparent ohmic conductor, making it an ideal interface for electronic connectivity, whilst limiting light absorption. These complimentary traits can provide the key ingredients for an optoelectronic device. These devices provide a potential platform for creating optoelectronic identification devices, whilst being of great interest for a number of other applications, including LEDs, quantum information processing and solar cells. A particularly interesting feature of this work is the use of CQDs capped with silica-shells. As of yet, the LB deposition of this type of nanoparticle has not been achieved, but they provide vast potential as they can be suspended in nontoxic solvents which allows for the increased flexibility of applications where toxicity is a concern. Here, the use of electrospray facilitates the LB deposition of silica capped CQDs for the first time. 


\subsection{Materials for an Optoelectronic Device Architecture}

\subsubsection{An Introduction to Colloidal Quantum Dots}

In brief, a colloidal quantum dot is a crystal of sufficiently small size such that quantum confinement starts to govern the properties of the system. When encapsulated within a different material, they are also commonly called nanoparticles (NPs); these labels will be used interchangeably in this chapter. The earliest studies on CQDs came in the early 1980s, when they were employed in water splitting and photocatalysis applications ${ }^{1}$. Along with these investigations, works by L. Brus et al. and A. Efros et al. explored the optical properties of these materials both in suspension and in a dielectric matrix ${ }^{2,3}$. It was found that the observed effects could only be explained through the requirement that quantum confinement was present in the system. For example, it was demonstrated that the optical absorption edge had shifted to higher energies with decreasing particle size whilst discrete peaks had become visible in the spectra. Typical CQDs range from 1-100 nm in each of their lateral dimensions, allowing tunability of the energy levels contained within the system and hence the emission wavelength. The three-dimensional confinement of these system often lend them the name, 'artificial atoms', as they display similar characteristics. However, owing to their increased size, CQDs are much easier to address whilst being readily mass-produced in colloidal suspensions and as such provide an interesting building block to build nanoscale systems for a range of applications. Although the size of the system provides adequate tuning, the optical properties of these systems are also dictated by the shape and composition. For example, it has been demonstrated that NPs with shapes deviating from spheres, such as nanorods and nanotetrapods can be produced with different resulting properties ${ }^{4,5}$. Moreover, the chemical nature of the surface plays a great role in determining the properties of the system and can result in the CQD being a good, or bad, light emitter. For example, it has been shown that the core of a NP can be encapsulated with a material with differing band gap and band offsets, such that both the electrons and holes are confined in the same layer. The result of this is a type-I structure, leading to the excitons being confined within the core, enhancement of the wavefunction overlap, and efficient light generation ${ }^{6,7}$. Likewise, this type of system can minimise the effect of traps 
at the interface, reducing the likelihood of non-radiative recombination ${ }^{8}$. However, encapsulation in a material of differing band gap and offsets in which electrons and holes are confined to different layers of the structure results in a type-II system being formed, with the resulting exciton being created such that the electron and hole are confined separately in the core and shell, respectively. This reduces the light output as the wavefunction overlap is reduced, as the carriers must cross the core-shell interface to recombine, and leads to long radiative lifetimes ${ }^{9}$. Although a large range of semiconducting nanoparticles exist in the literature, the work here focusses on the use of CdSe CQDs encapsulated in silica shells.

\subsubsection{CdSe Nanoparticles}

Cadmium-selenium NPs with extraordinary properties have been synthesized into near-monodisperse suspensions by wet chemical methods and have already been implemented into a number of applications for light emitting devices ${ }^{10,11}$. Using surfactants to encapsulate these nanoparticles offers a preventative step against aggregation whilst providing electron passivation. As an example, a CdSe CQD encapsulated with a surfactant, trioctylphosphine oxide (TOPO), displays a quantum yield of approximately $10 \%$ at room temperature ${ }^{12}$. However, this surfactant is extremely dangerous, unstable and environmentally unfriendly. Therefore, alternatives have been suggested and by coating a CdSe core with a ZnS shell, a material with different band offsets and band gap, it has been shown that the quantum yield can be improved to a value of over $60 \%^{13}$.

Although these particles have been stabilised in non-aqueous environments, the use of a versatile range of solvents and ligands has been elusive as this effects the photophysical nature of the nanoparticles. Although bright luminescence has been observed from CdSe CQDs fabricated with lesstoxic sources ${ }^{14}$, the challenge remains to create water-soluble systems that display effective colloidal stability, photostability and efficient photoluminescence. To this end, there are two main methods in the literature; the first utilises an organic coating which can comprise of either polymers, micelles or thiol groups $s^{15,16,17}$. The second method lends the techniques that have been routinely applied to metal 
NPs, in the form of silica coatings ${ }^{18,19}$. The advantages of using silica lies in its ability to provide an inert layer that can protect the optical properties of the system whilst allowing the particles to be soluble in water, giving the system a reduced toxicity.

\subsubsection{Building Heterostructures}

To create an optoelectronic device incorporating NPs, the use of graphene contacts has been suggested, similar to the work by Lu et $\left.a\right|^{20}$. Schematics of a typical device architecture for application as a UNO/PUF or a solar cell can be seen in fig 6.1.

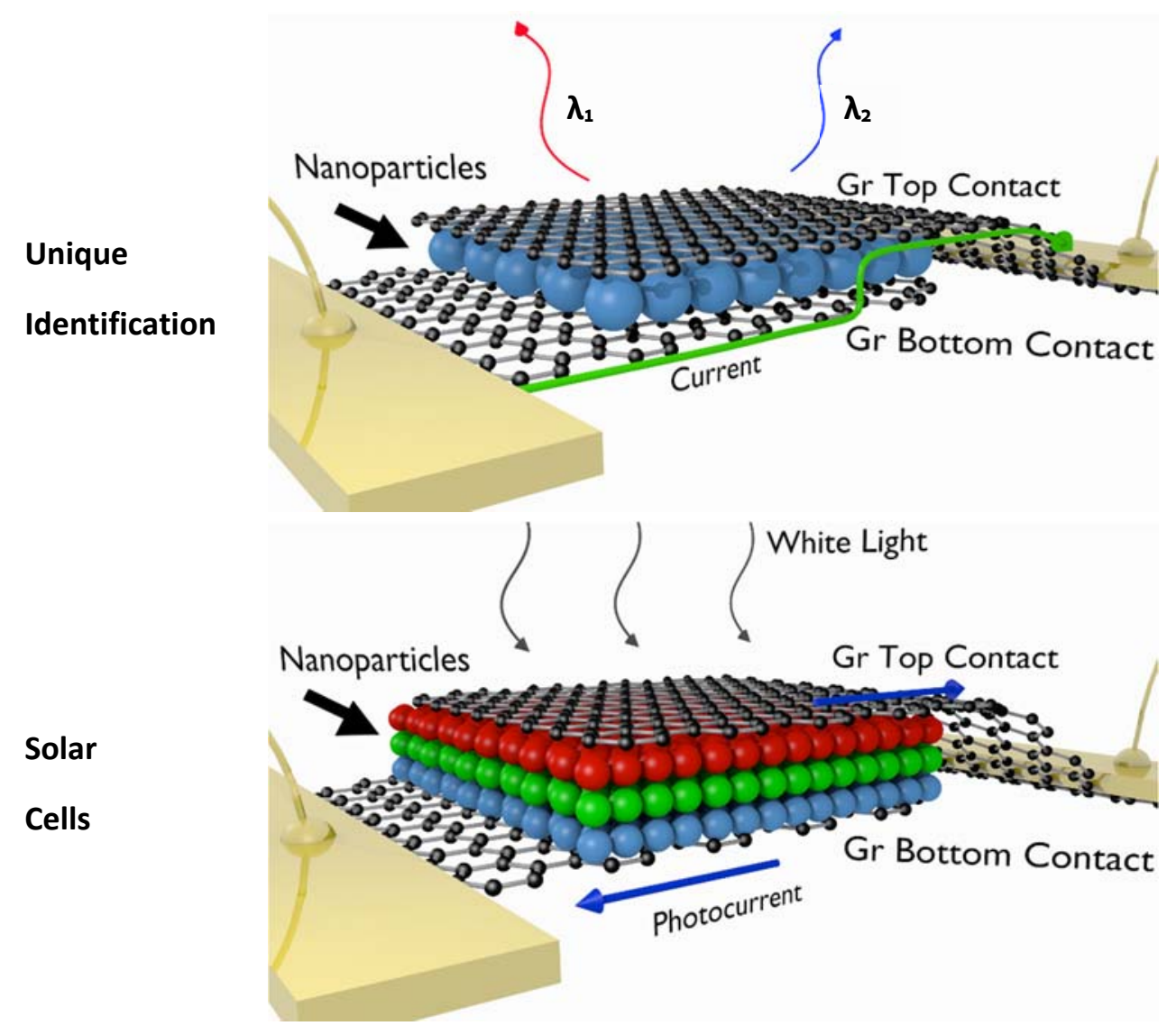

Figure 6.1: Schematic of a graphene/NP/graphene device for optoelectronic applications. The top and bottom images show the architecture envisaged for use in unique identification and solar cells, respectively. The composition-dependent emission would result in wavelength shifts over the surface, whilst a larger order heterostructure could absorb a wide range of wavelengths for photocurrent generation by incorporating a number of layers with different band gaps.

Here, the fabrication of 1 monolayer of CQDs between graphene sheets would show variability in the emission wavelength due to the compositional variation at the graphene-NP interfaces, enabling 
unique identification. Optoelectronic identification is extremely useful for checking the authenticity of an electronic component, capable of providing power to the device and remove the need for an external excitation source. Alternatively, building larger heterostructures with different layers of size selected CQDs would allow broadband collection of white light for efficient solar cell applications. A key difference in this work compared to the work in [20] is the use of silica-terminated CdSe NPs, which provide less-toxic chemicals to be used for suspension and handling. Furthermore, the Langmuir-Blodgett technique is employed in this work, a technique that has so far been unsuccessful for silica coated CQDs. This facilitates control over the close-packing of the CQDs at the air-water interface and can allow a uniform monolayer to be formed.

To create graphene contacts, CVD grown graphene has been used, although graphene can be effectively transferred via the Langmuir-Blodgett technique. The reasons behind this are that it provides a higher quality of material due to the vast range of optimisation procedures that have been achieved in this field. Furthermore, it allows the approximate positioning of the graphene sheet onto a substrate, allowing contact areas to be defined; whereas Langmuir-Blodgett deposition results in uniform coverage over the entire area. CVD graphene also provides a continuous sheet across large areas $(\sim \mathrm{cm})$ which gives reliable results when wanting to measure transport characteristics of such devices. Graphene is a convenient material in such an application, providing a flexible bonding platform that has an extremely high transparency, necessary for high light-output devices. It should be noted that the Langmuir-Blodgett technique could be used for all steps of such a heterostructure, and this will be explored in future work. As in the previous chapter, the use of electrospray for the deposition of material onto the air-water interface has facilitated the creation of thin films of NPs to be transferred onto substrates. In all the following experiments used here, the substrate that has been employed was a silicon wafer coated with $290 \pm 5 \mathrm{~nm}$ of $\mathrm{SiO}_{2}$, with or without a deposited graphene layer, as described in the text. All wafers were cleaned with acetone and IPA for 5 mins prior to deposition, followed by drying with $\mathrm{N}_{2}$ and an oxygen plasma clean (Diener Femto system, $98 \% / 2 \%$ $\mathrm{Ar} / \mathrm{O}_{2}, 15 \mathrm{~L} / \mathrm{h}$ flow rate) on full power for 5 minutes, to ensure hydrophilicity of the substrate. 


\subsection{Langmuir-Blodgett Films of CdSe/Si Nanoparticles}

\subsubsection{Nanoparticles at the Air-Water Interface}

All the nanoparticles that are used for creating Langmuir films in the following experiments have CdSe cores of varying sizes (the specific structure can be found in ref. [21] and consists of a CdSe core encapsulated with an alloy of CdSeS/ZnSeS and further encapsulated with ZnS; for simplicity the NP is referred to as CdSe in all cases here), surrounded by dichlorodiphenyltrichloroethane (DDT) ligands, and finally encapsulated with silica shells. Furthermore, all dispersions are suspended in ethanol, allowed by the use of a silica shell. To test the efficacy of using electrospray for deposition of nanoparticles onto the air-water interface, a number of different sized NPs were tested.

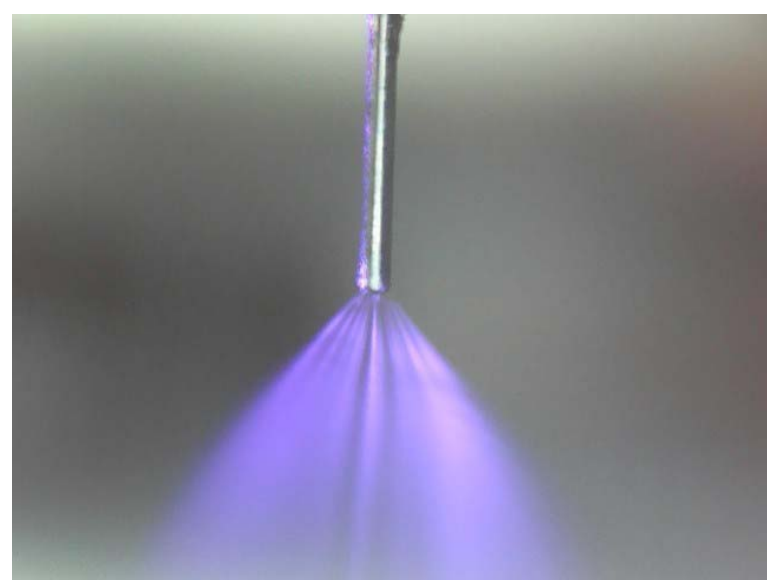

Figure 6.2: Electrospray of nanoparticles operating in the multi-jet mode, a higher order regime of the cone-jet mode. Spraying in this mode allowed for a much higher flow rate of the solution.

For a typical experiment, the trough was cleaned and prepared with a water subphase in the routine way, and a $100 \mu \mathrm{m}$ needle was used for electrospray. The electrospray was performed at $5 \mathrm{kV}$, at a distance of approximately $5 \mathrm{~cm}$ above the water surface resulting in the formation of the 'multi-jet mode'; this allowed for a much faster flow rate. The shape of this mode can be observed in fig 6.2. To take isotherms, different NPs were chosen and diluted with ethanol such that the material deposited onto the surface always had a concentration of $1.5 \mathrm{mmol} / \mathrm{L}$. The addition of extra ethanol into the CQD solution was to allow ease of electrospraying whilst ensuring that no blockage of the needle took place. Once all material was deposited, the barriers were moved towards the centre of the trough at a rate of $5 \mathrm{~mm} / \mathrm{min}$ and for initial experiments the barriers were moved such that the final area was 
$\sim 35 \mathrm{~cm}^{2}$, or until the critical pressure was reached.

The data in fig 6.3 shows two typical isotherms from different sizes of NPs deposited in identical ways. The red (blue) trace shows nanoparticles with estimated sizes of $3 \mathrm{~nm}(4.5 \mathrm{~nm})$ for the core diameter and a $12 \mathrm{~nm}(8 \mathrm{~nm})$ silica shell thickness. It was observed that the isotherms showed very similar shapes highlighting alike interaction mechanisms at the interface, but reached different critical pressures, which is likely due to the different diameters of the NPs. Furthermore, the smaller sized particles used here showed an increase in pressure at much larger areas, it is suggested that this could be due to the increased surface area to volume ratio, giving the particles a higher surface energy and therefore a slightly enhanced interaction with neighbouring nanoparticles. Moreover, the solution with larger NPs could contain uncoated nanoparticles (just the CdSe CQD), and this could affect the ability of the film to form a solid layer, causing the surface pressure to increase quicker for the smaller

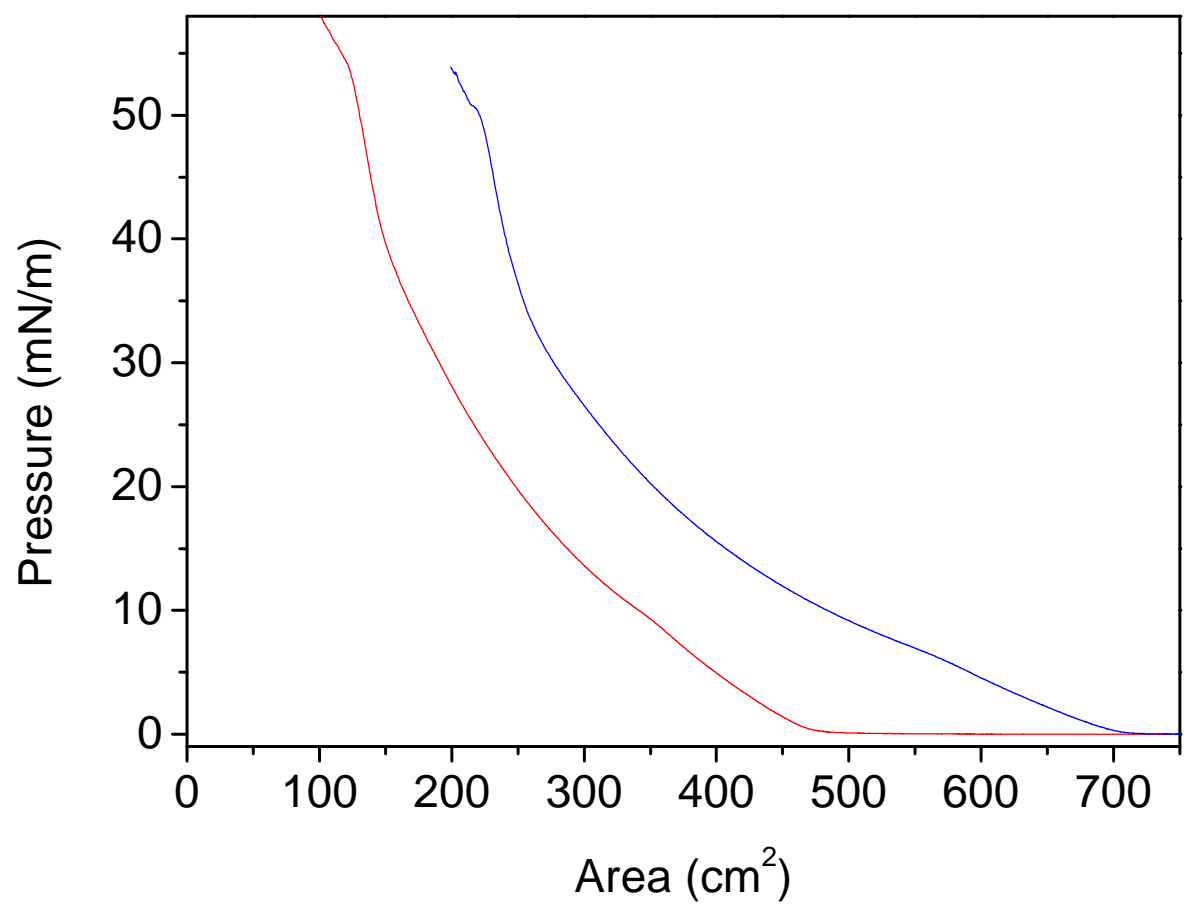

Figure 6.3: Pressure-area isotherms of two types of CdSe, silica capped NPs with core (shell) sizes of $3 \mathrm{~nm}(12 \mathrm{~nm})$ and $4.5 \mathrm{~nm}(8 \mathrm{~nm})$ for the red and blue trace, respectively. Similar isotherms indicated a similar surface-interaction mechanism whilst the variances are mainly attributed to the difference in NP diameter. 
particles. A change in gradient at high pressures indicates that the critical pressure has been reached and the gradient of the line just before this represents the 'solid' phase,

To further investigate the formation of nanoparticle films on the water surface, Brewster angle microscopy (BAM) was performed. The data in fig 6.4 shows how the interface between the NPs and the water changes as the pressure is increased, for the two different types of nanoparticles in fig 6.3. The BAM images were recorded every 15 seconds once a minimum pressure of $1 \mathrm{mN} / \mathrm{m}$ was reached. The technique here provides two main advantages, first of all it allows the user to discern whether or not nanoparticles are present on the surface, especially difficult with weak emitting species that show no observable colour. Secondly, it provides an insight into the transfer process by investigating similarities and differences between the film on the surface and on a substrate.

The data in fig $6.4 \mathrm{a}$ corresponds to the blue trace in fig 6.3 , whereas $6.4 \mathrm{~b}$ corresponds to the red trace. It was observed for the small particles that there appears to be a good film coverage on the surface of the water even at relatively low pressures; albeit with wide visible gaps. With this particular type of nanoparticle, something very interesting happens as the barriers are compressed and forces the pressure to rise above $25 \mathrm{mN} / \mathrm{m}$, with a snowflake-like pattern beginning to appear on the water surface (highlighted in the inset in fig $6.4 \mathrm{a}$ at $25 \mathrm{mN} / \mathrm{m}$ ). This pattern shows very similar characteristics to other monolayers being formed at the air-water interface, and results from phase separation due to the interaction between the two interfaces ${ }^{22}$. However, the striking difference here is that the snowflake-like patterns are being formed by the water surface and not by the NPs, of which no evidence has been found in the literature for this type of behaviour. This phase separation behaviour is of great interest for the physical mechanism of film formation and is still under investigation. The current hypothesis is that the phase separation is due to unattached ligands that can float on the water surface. These ligands can aggregate forming localised regions in which no nanoparticles reside, explaining the large gaps in the film. Once the pressure of this type of nanoparticle has been increased to $40 \mathrm{mN} / \mathrm{m}$, a uniform film can be seen across the entire image. 
(a)
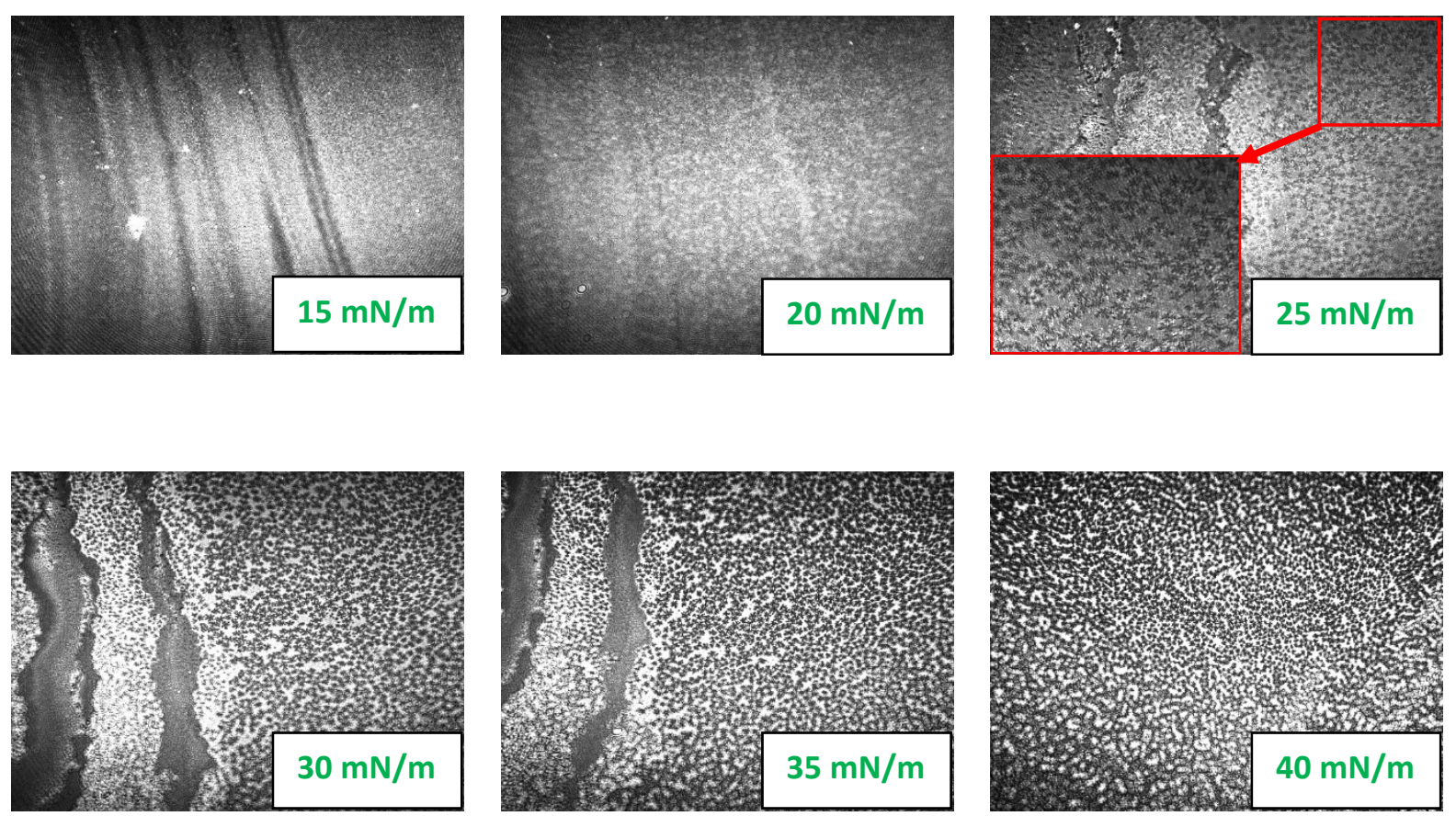

(b)
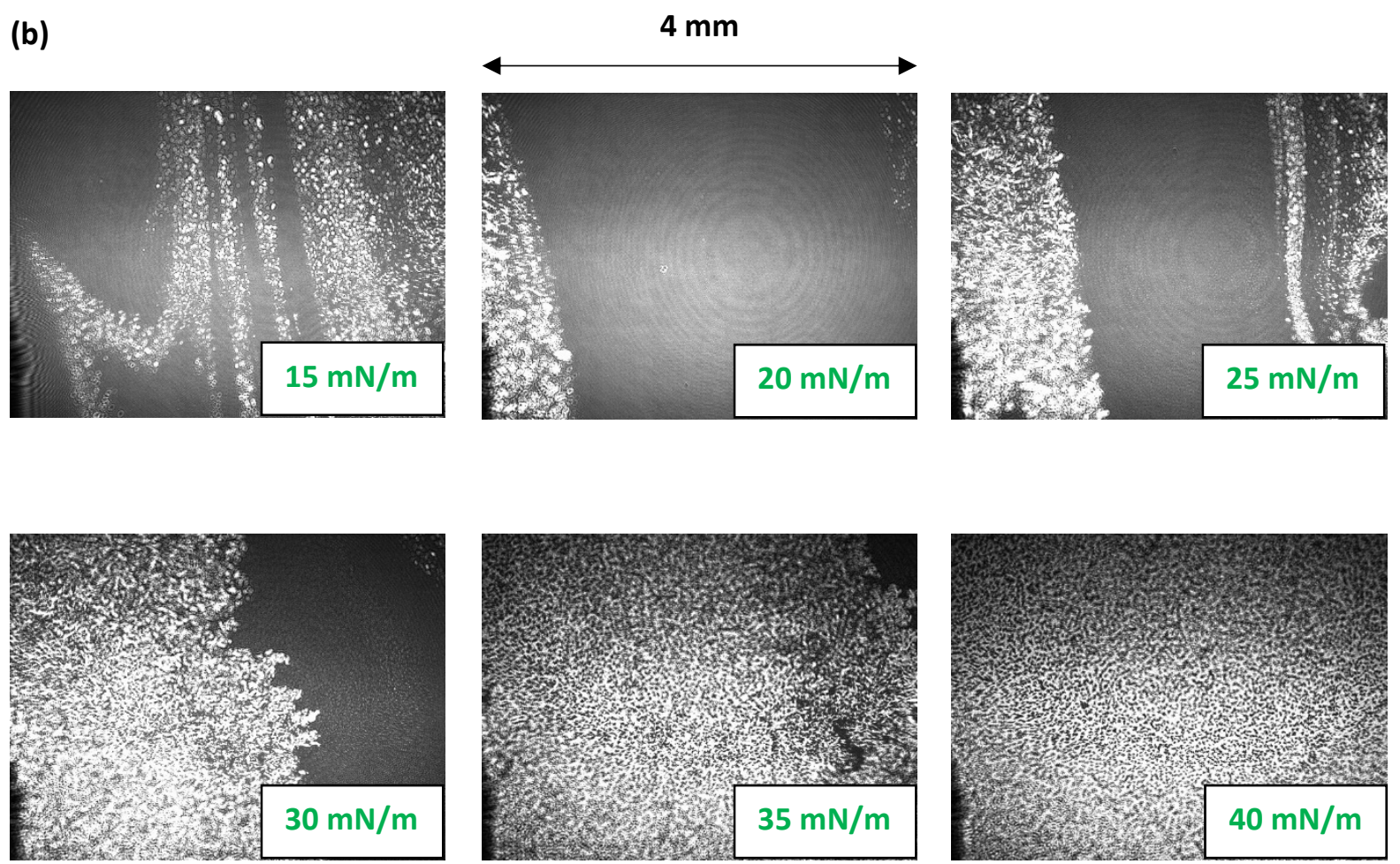

Figure 6.4: Brewster angle microscopy images of the air-water interface for (a) the $4.5 \mathrm{~nm}$ core nanoparticles and (b) the $3 \mathrm{~nm}$ core particles as the pressure is increased from $15-40 \mathrm{mN} / \mathrm{m}$. The formed films are relatively uniform as different regions across the surface appear very similar, although individual regions have large discontinuities (highlighted by the inset in the top-right panel of (a)). Widths of all images is $4 \mathrm{~mm}$. 
In the definition of 'uniform coverage' here, it relates to the homogeneity that is observed over large scales ( millimetres), with higher magnifications it is expected that the film will be much less uniform. There is some evidence for this in the inset of fig $6.4 a$ at $25 \mathrm{mN} / \mathrm{m}$. Importantly, it has been observed that a uniform coverage has appeared for both these nanoparticles at high pressures, indicating the formation of a 'solid' film.

\subsubsection{Deposition of a Langmuir-Blodgett Film}

To transfer a film of nanoparticles, a clean silicon wafer was placed into the subphase before deposition, so that only 1 layer could be transferred. For the Langmuir-Blodgett films, a deposition pressure between 40 and $45 \mathrm{mN} / \mathrm{m}$ was chosen as this was just before the critical pressure observed on the isotherm for both species of NP tested. Hence, this should allow the preparation of a film that is in the solid phase. Once the barriers were compressed, the pressure was controlled by slowly changing the barrier position and the dipping experiments were performed using a pulling speed of $0.5 \mathrm{~mm} / \mathrm{min}$ to allow controlled transfer to take place. Following deposition, the films were left to dry in vacuum to remove adsorbed water molecules. A hot plate cannot be used in the case of these NPs, as high temperatures can cause degradation of the DDT ligands.
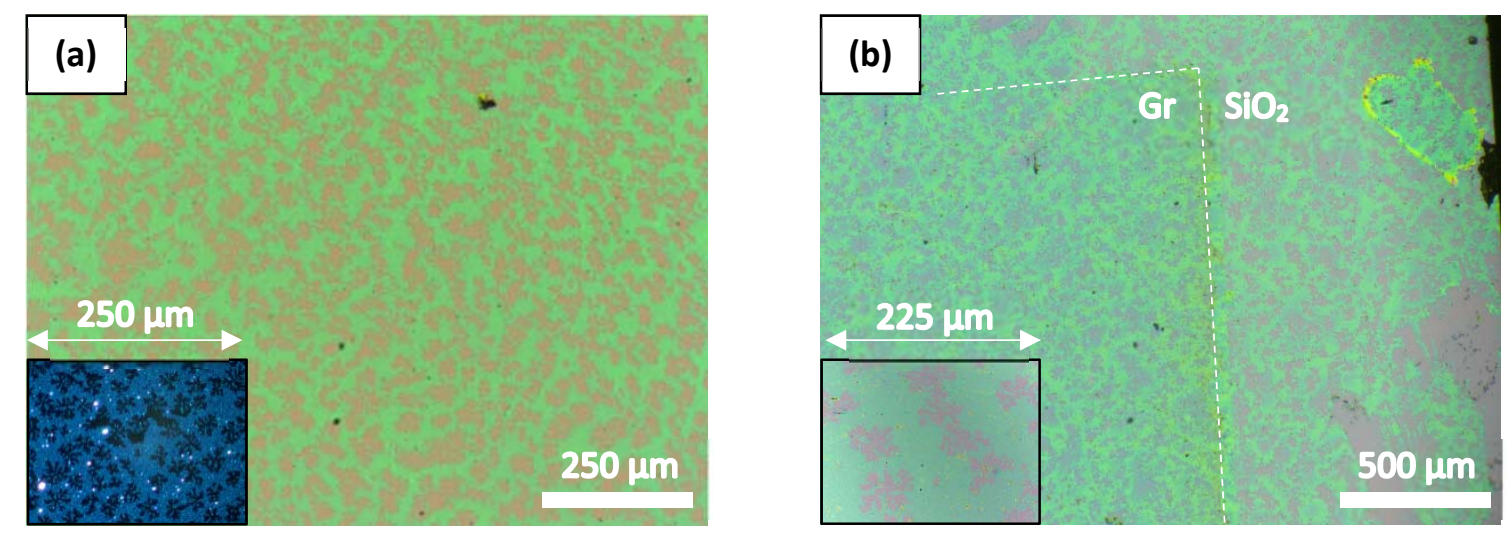

Figure 6.5: Optical images of deposited films at $40 \mathrm{mN} / \mathrm{m}$, showing (a) $4.5 \mathrm{~nm}$ core and (b) $3 \mathrm{~nm}$ core nanoparticles, demonstrating the homogeneous, but not continuous, coverage of NPS on both silicon and graphene. Insets: (a) shows a $20 \mathrm{mN} / \mathrm{m}$ film, (b) zoomed-in region of the film.

The images in fig 6.5 show how the films appear optically after deposition of $40 \mathrm{mN} / \mathrm{m}$ films of the two species of nanoparticle. Fig 4.5a shows the deposition of the smaller nanoparticles onto $\mathrm{SiO}_{2}$ and 
it can be seen that in confirmation of the BAM results, this snowflake-like pattern have appeared within the film even at these high pressures. This can be compared to the inset which shows the same film deposited at $20 \mathrm{mN} / \mathrm{m}$, where these snowflake-like features are much further apart.

To confirm that the NPs transferred just as well onto graphene, $\mathrm{SiO}_{2}$ substrates were prepared using a graphene transfer protocol. The graphene used for this process was grown on copper foil that had a $25 \mu \mathrm{m}$ thickness. Copper has a negligible carbon solubility at high temperatures, and as such adsorbed hydrocarbon molecules on the surface results in graphene growth ${ }^{23}$. Ruthenium and nickel can also be used, but here the graphene grows via segregation of carbon from the bulk to the surface due to the far larger carbon solubility ${ }^{24}$. The graphene was grown via chemical vapour deposition (CVD) as described in ref. [25]. Using the CVD grown graphene requires transfer onto a suitable substrate and was carried out via the following process. In this case, $\mathrm{SiO}_{2}$ was used as the substrate was required to be insulating for electrical characterisation.

To remove the graphene from the copper, a wet etching process was used. Firstly, a layer of polymethyl methacrylate (950 PMMA A $9^{26}$ ) was spin-coated onto the copper/graphene foil at a speed of $4000 \mathrm{rpm}$, giving a thickness of $1.5 \mu \mathrm{m}$, then allowed to dry. The graphene on the underneath of the copper foil is then removed via a 30 second oxygen plasma etch, necessary as the graphene residues from this side can contaminate the graphene sheet to be transferred. This PMMA/graphene/copper structure is then allowed to float on an ammonium persulfate (APS) solution until the copper is fully etched away. Whilst the remaining PMMA and graphene is then removed from the surface using a glass slide and subsequently cleaned using a number of DI water baths. To transfer this film onto a substrate, the film is left to float on the water surface before scooping it up with the target substrate and guiding the film with a syringe needle. Following this, a water film remains trapped between the graphene and the substrate which must be removed as this affects the cleanliness of the sheet and its adherence to the substrate. If this step is ignored, the graphene will be detached when the PMMA is removed. The removal of water is done by placing the substrate into a vacuum chamber at $10 \mathrm{mbar}$ 
overnight, and to further promote adhesion, a $200^{\circ} \mathrm{C}$ bake is applied prior to removal of PMMA in acetone. This process is summarised in fig 6.6.

The image in fig $6.5 \mathrm{~b}$ shows the larger nanoparticles deposited onto an $\mathrm{SiO}_{2}$ substrate with a pretransferred graphene sheet. Here, the interface between the substrate and the graphene is highlighted in the figure. It was observed that the coverage on the $\mathrm{SiO}_{2}$ and the graphene is very similar, suggesting that the deposition of material is only subtly effected by the introduction of graphene.

(a)

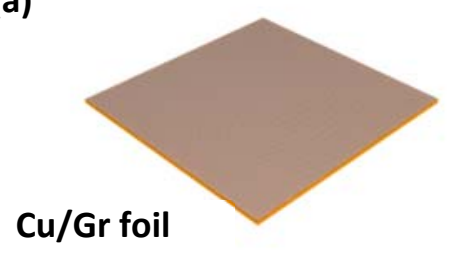

(b)

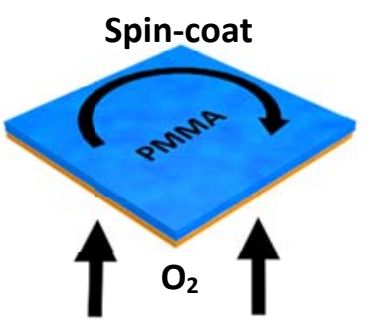

Plasma

(d)

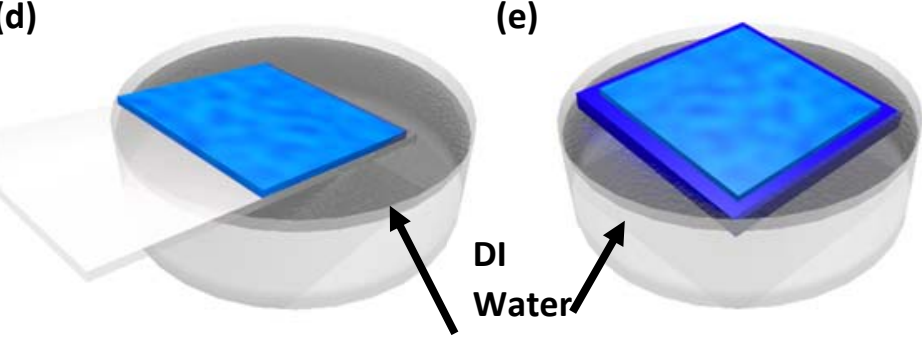

(c)

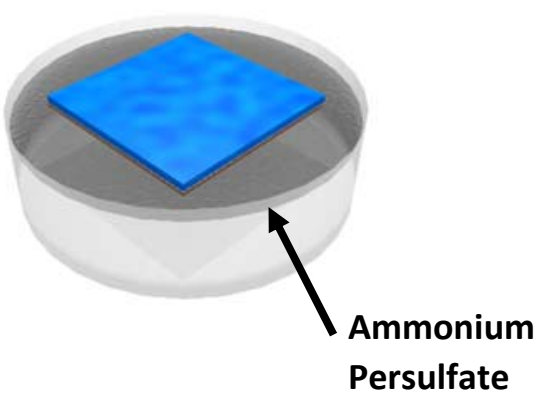

(f)

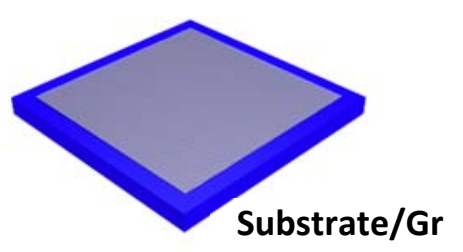

Figure 6.6: Summary of graphene transfer process: (a) graphene on a copper substrate, (b) PMMA spin-coat added and oxygen plasma etch to remove bottom graphene layer, (c) copper etch in APS leaves a PMMA/Gr membrane, (d) removal to glass slide and DI rinse to remove excess APS, (e) transfer to substrate from DI water by 'scooping' up the membrane, (f) Graphene on target substrate after PMMA removal with acetone.

A number of different areas within the transferred films of both types of nanoparticles on the two different substrates was inspected with the optical microscope, with the contrast differences highlighting regions with NPs/no NPs, and the following was found. On the $\mathrm{SiO}_{2}$, the smaller (larger) nanoparticles covered $67.2 \pm 2.10 \%(63.55 \pm 3.20 \%)$ of the area of the substrates, whereas on graphene they covered $71.15 \pm 1.87 \%(64.83 \pm 2.75 \%)$ of the surface. These values agree well within 
two standard errors suggesting that the nanoparticles deposit onto either substrate with no preferential adherence to one or the other; this may be because the nanoparticles effectively 'see' the $\mathrm{SiO}_{2}$ underneath the graphene. Interestingly, the average coverage on graphene seemed to be larger each time, which is useful when it comes to the fabrication of devices. To increase this coverage towards $100 \%$, the film at the air-water surface must be improved and will be partly investigated later in the chapter. It can clearly be observed that the film is discontinuous over a large scale, with many gaps, however for fabrication of a device only a $5 \times 5 \mu \mathrm{m}^{2}$ area would be required, which is easily obtainable (see inset to fig $6.5 b$ ).

\subsection{Analysis of Nanoparticle Films}

\subsubsection{Investigation of Film Homogeneity}

To explore the uniformity of the deposited nanoparticle films, a number of methods were employed. First of all, scanning electron microscopy was used to achieve an insight into the order of the films on a number of length scales whilst providing a method of approximating the diameter of the nanoparticles to see if they comply with the expected values. All the following transferred films were made through depositing 1 layer at a pressure of $40 \mathrm{mN} / \mathrm{m}$ and a dipping speed of $0.5 \mathrm{~mm} / \mathrm{min}$. The images shown in fig 6.7 show a comparison of the deposited films of NPs on silicon for the two sizes of particle studied.
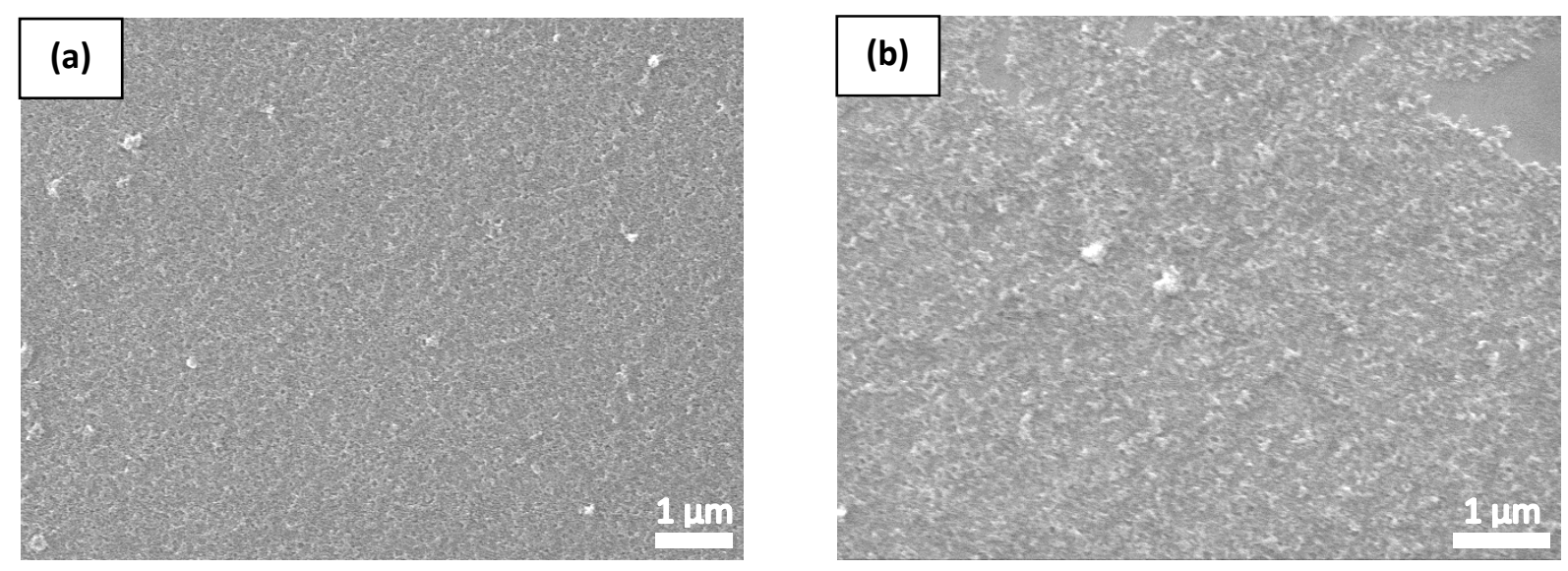

Figure 6.7: Comparison of the two types of nanoparticle on an $\mathrm{SiO}_{2}$ substrate, with (a) the $3 \mathrm{~nm}$ core and (b) the $4.5 \mathrm{~nm}$ core nanoparticle. A greater amount of inhomogeneity was seen for the $4.5 \mathrm{~nm}$ core NPs, resulting in the $3 \mathrm{~nm}$ core NPs being chosen for subsequent heterostructure creation. 
It was observed that the larger nanoparticles in fig 6.7a showed a more homogenous coverage, with the smaller nanoparticle film exhibiting many regions where the film was evidently not well ordered; this can be inferred by the greater contrast changes in fig $6.7 \mathrm{~b}$. In both films, large imperfections in the film were seen, thought to arise from leftover PMMA from the graphene transfer process. Due to this observation, the production of heterostructures was completed using the larger NPs.
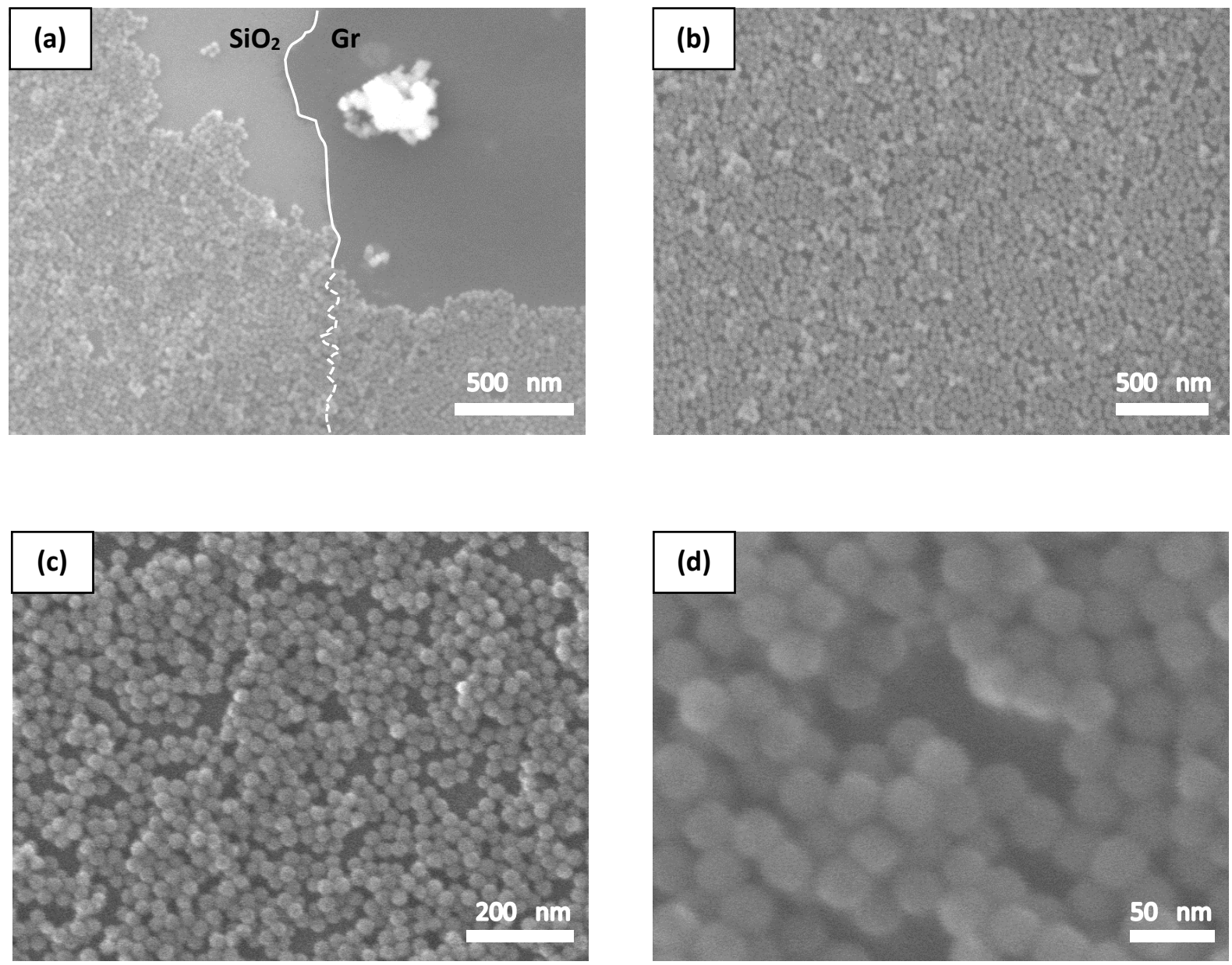

Figure 6.8: Investigations into the nanoparticle film showing (a) the uniformity of the transferred film across the $\mathrm{SiO}_{2}$ /graphene interface, confirming a negligible effect of the substrate on the NP film (the interface is highlighted), (b) a NP film transferred by pulling the substrate from the subphase at an angle of $110^{\circ}$, (c-d) $110,000 x$ and $350,000 x$ magnification, respectively, allowing exploration of gaps and size distributions of the NP film.

As suggested from the optical images in fig 6.5, the difference in deposition of material across the interface between silicon and graphene is negligible, with NP coverages being equal within errors on the measurement. This has been confirmed in the SEM measurements, highlighted in fig 6.8a. Here, 
the grey/black transition in the top-right of the image is the silicon/graphene interface. To guide the reader, a line that highlights this interface has been shown. The interface cannot clearly be discerned below the nanoparticles, and the dashed line provides an estimate for the continuation of the graphene sheet. The nanoparticles spanning across the interface have been observed to show no disorder even though there is a ridge at the edge of the graphene sheet formed during transfer. This is extremely important for the creation of heterostructures, as the NPs need to be well adhered to the graphene for a device to be fabricated.

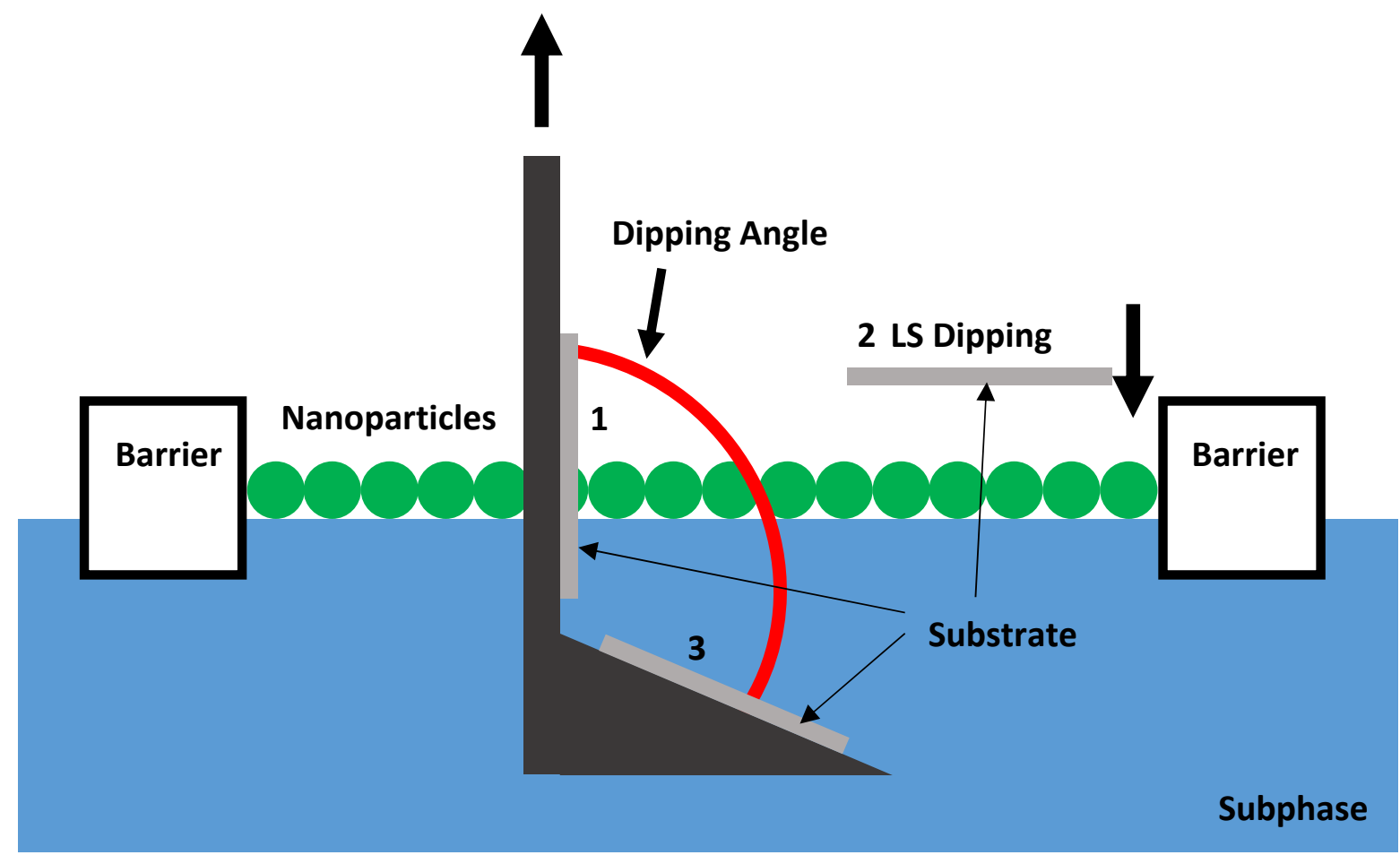

Figure 6.9: Alternate methods of transferring NP films, highlighting; 1 . The standard LangmuirBlodgett (LB) method, 2. The Langmuir- Schæfer (LS) method and 3. The angled LB method.

To test the quality of the film transfer under different transfer conditions, the dipping angle in which the substrate was pulled up through the water interface was changed. A number of angles were tried between $90^{\circ}$ and $180^{\circ}$ to the normal; with $180^{\circ}$ being the conventional vertical dipping orientation. This is illustrated in fig 6.9, along with conventional LB. This seemed to have very little effect on the formation of the film on the substrate. For example, fig $6.8 \mathrm{~b}$ shows an example of a film deposited using an angle of $110^{\circ}$ to the normal, which has been shown to give better film qualities for similar 
types of nanoparticle ${ }^{27}$. However, it can be seen that the deposited film shows negligible difference to the one deposited vertically at the same pressure in fig 6.7a. Therefore, for all further film depositions, the simpler orientation of a vertical dipping angle was used. It should also be noted that the LangmuirSchæfer (LS) method was also attempted for deposition of nanoparticles. The LS technique corresponds to taking a 'stamp' of the film by bringing the substrate down in parallel to the air-water interface until it comes into contact, and is subsequently pulled upward (see fig 6.9). However, this gave highly non-uniform films which is suggested to be due to the large amount of excess water left behind following this dipping method.

Figs $6.8 \mathrm{c}$ and $6.8 \mathrm{~d}$ show the NP film at higher magnifications. It was observed that the film is highly disordered, with no clear areas where the nanoparticles have begun to close pack over areas larger than a few NPs in size. The gaps in the film on this scale are thought to arise from adsorbed molecules that are attached to the surface during deposition which are subsequently removed, leaving openings in the film. Furthermore, it can be seen that although only one dip was performed, there are many regions that are greater than a monolayer. The mechanism behind this in not yet fully understood, one suggestion is that the particles could be ordering in a stacked manner on the water surface due to over-compression. Another possibility is the rapid accretion of NPs onto the substrate as it is being

(a)

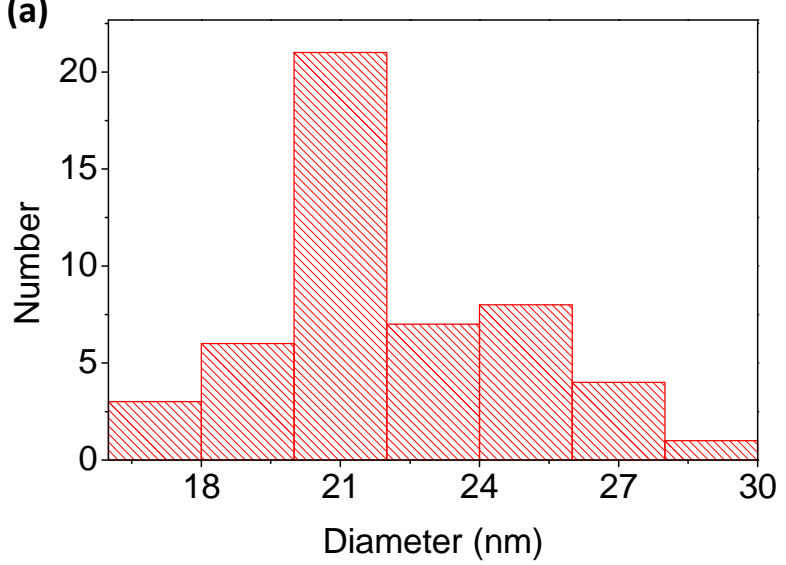

(b)

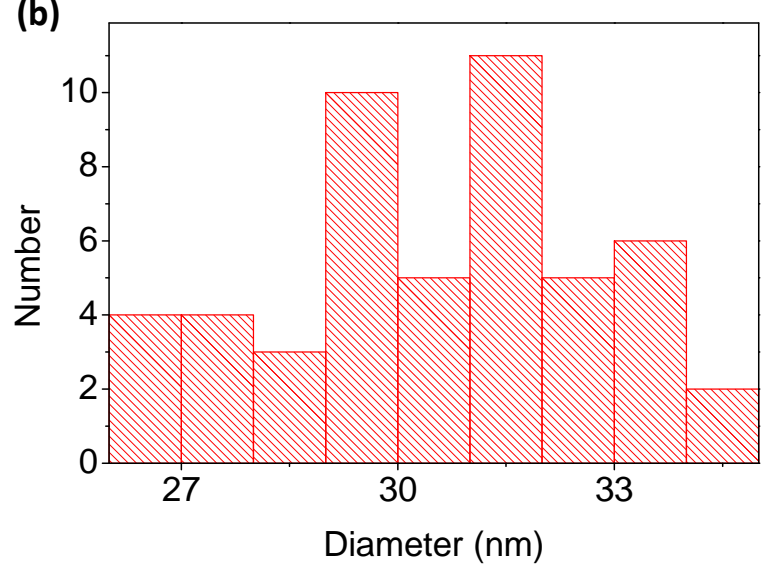

Figure 6.10: Histograms of the nanoparticle diameter extracted from SEM images, showing (a) $4.5 \mathrm{~nm}$ core and (b) $3 \mathrm{~nm}$ core nanoparticles. The average NP diameter was found to be $22.2 \pm 0.4 \mathrm{~nm}$ and 30.5 $\pm 0.3 \mathrm{~nm}$, respectively, in good agreement with the estimated core and shell values predicted from the chemical synthesis. 
pulled out of the subphase, due to the slow dipping speed. The appearance of these higher regions could actually be of benefit to fabricated optoelectronic heterostructures. To this end, they could prevent the graphene contacts from short-circuiting due to the gaps that appear in the NP film. If it is found that this is the case, the LB method could be used to deposit multiple layers of NPs with ease.

Using the SEM images, the diameter of the nanoparticles was estimated for the two species used thus far. This was done by measuring the diameter of 50 different nanoparticles for both species by fitting circular shapes to nanoparticles in images where the edges could easily be discerned (such as in fig $6.8 \mathrm{c} / \mathrm{d})$. Although the nanoparticles are not perfectly spherical, this allowed a good estimation of the nanoparticle diameter. Fig 6.10 shows histograms of the measured sizes of nanoparticles for the two species. The average nanoparticle size was calculated to be $22.2 \pm 0.4 \mathrm{~nm}$ for the small particles and $30.5 \pm 0.3 \mathrm{~nm}$ for the large particles. This is in good agreement with the estimated values for the cores plus the silicon shells, predicted by the chemical synthesis as $4.5 \mathrm{~nm}+(2 \times 8 \mathrm{~nm})=20.5 \mathrm{~nm}$, and $3 \mathrm{~nm}$ $+(2 \times 12 \mathrm{~nm})=27 \mathrm{~nm}$. However, these values do not take into account the lengths of the DDT ligands attached between the core and the shell, and can account for the discrepancy between measurements. Furthermore, the larger nanoparticles seem to have a much larger spread in diameters, which would be of particular interest in unique identification as this would change the emission wavelength.

\subsubsection{Stacking of the Film}

To understand the thicknesses of the deposited films, atomic force microscopy (AFM) was employed. It was found that the nanoparticles were not well adhered to the substrate or the graphene, likely due to the minimal interaction between the surfaces due to their spherical shape. This made measurements extremely difficult and slow scan speeds had to be used such that the nanoparticles weren't dragged around by the tip, which destroys the measurement. Furthermore, this made the use of contact-mode AFM almost impossible, therefore tapping-mode was used which resulted in a decreased resolution but allowed for an estimation of the film thickness. The settings required for 
such measurements were optimised and a scan speed of $0.3 \mathrm{~Hz}$ and a drive amplitude of $6.71 \mathrm{mV}$ were found to give the best results; these values are significantly less than those used in the previous chapter for measuring $\mathrm{MoS}_{2}$. The other parameters were similar, with an amplitude set-point of $335.42 \mathrm{mV}$ and samples per line of 384 . The AFM results can be seen in fig 6.11 . Here the smaller nanoparticles were imaged whilst on silicon, which as discussed should give a representative estimation of the thickness of NPs on the graphene (due to their coverage similarities). This provides a much simpler measurement as the uncoated silicon has a low surface roughness in comparison to the graphene so allows a 'zero thickness' to be withdrawn effectively.
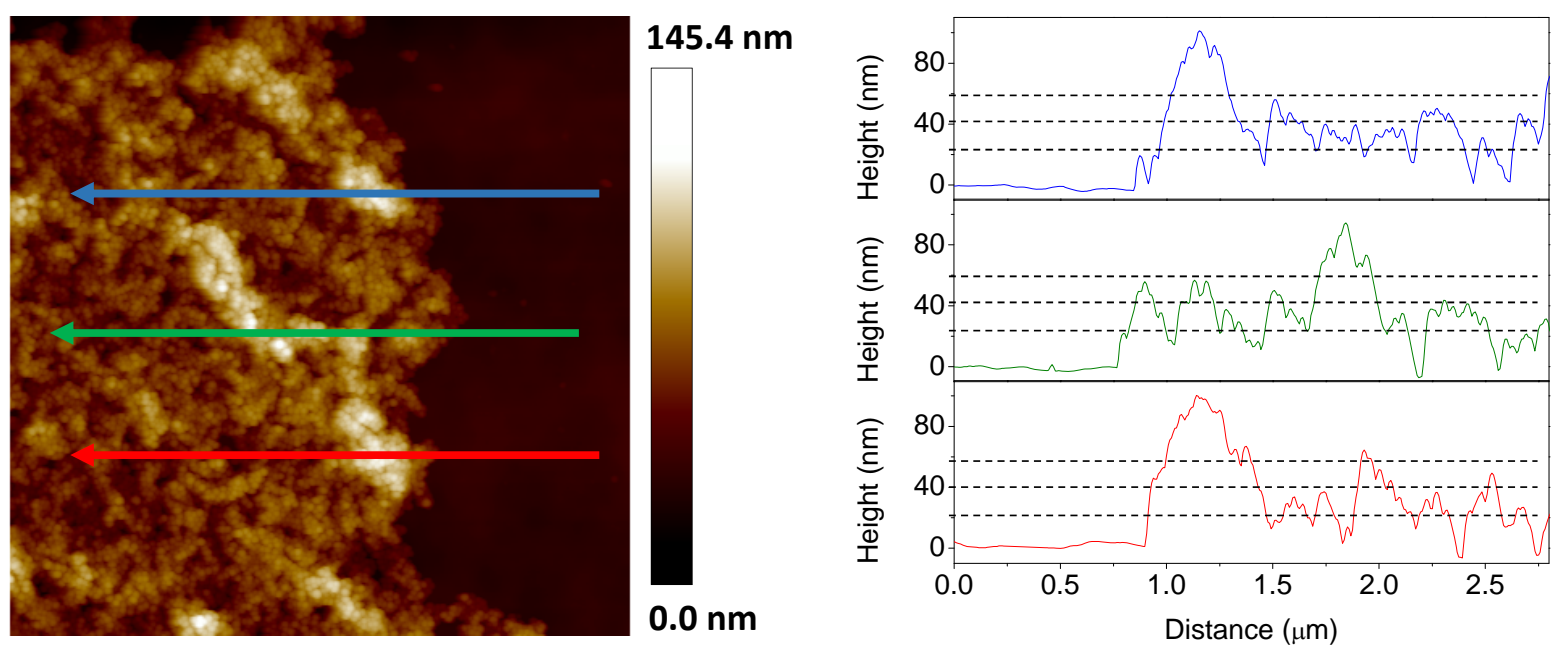

Figure 6.11: AFM topography image of a typical nanoparticle film deposited at $40 \mathrm{mN} / \mathrm{m}$ showing height variations throughout the film. Three height profiles were taken along the depicted lines, denoted by blue, green and red. The dashed lines in the profiles indicate the expected height of a nanoparticle film of 1, 2 and 3 monolayers if stacked via FCC or HCP.

The profiles in fig 6.11 are extracted from the lines drawn on the AFM image once the silicon area has been set as 'flat'. In particular, these profiles were drawn to look at the regions that are clearly higher than the rest of the film. Although many regions of approximately $20 \mathrm{~nm}$ can be seen, according to one monolayer of nanoparticles, there is a large range of heights across the film, with the maximum height above the silicon surface being measured as $\sim 105 \mathrm{~nm}$ in this particular image.

To approximate how high these films are in terms of nanoparticle layers, a close-packing nature for the stacking of the nanoparticles is assumed. In particular, face-centred-cubic (FCC) or hexagonal 
close-packed (HCP) packing is presumed, as the order of the first layer is observed to display this type of packing nature in some regions (see fig 6.8c). Using this approximation and the calculated diameter of the nanoparticles, $22.2 \pm 0.4 \mathrm{~nm}$, allows the heights of the different numbers of layers in the film to be estimated; this would be the same regardless of whether the film stacks in the FCC or HCP method. This gives values for $2,3,4,5$ and 6 monolayer stacks of $40.8 \pm 0.7 \mathrm{~nm}, 58.5 \pm 1.1 \mathrm{~nm}, 77.6 \pm$ $1.4 \mathrm{~nm}, 94.8 \pm 1.7 \mathrm{~nm}$ and $112.8 \pm 2.0 \mathrm{~nm}$, respectively. Fig 6.11 and these estimations suggest that in some areas of the film, the particles are stacking as high as 6 layers tall, an interesting observation whose mechanism is as yet not fully understood. However, these values do not show particularly good agreement with the heights across the AFM height profiles, highlighted by the dashed lines in the height profiles corresponding to 1, 2 and 3 monolayers using the above values. Thus, it is suggested that the stacking of the films of NPs is highly disordered with no simple way of understanding the nature of the stacked nanoparticle layers that are attached to the substrate and how it has ordered. However, through analysis of a number of images of NPs on silicon, looking at the regions where nanoparticles are present, an average height of $38.4 \pm 2.4 \mathrm{~nm}$ was found for the as-deposited films. This value tends to point towards the average coverage being that of two layers of nanoparticles.

\subsection{Creation of Heterostructures}

\subsubsection{Adding the Top Graphene Contact}

Using the methodology described in fig 6.6, a top graphene contact was added to a sample consisting of graphene and nanoparticles on a silicon substrate. The graphene was placed onto the sample such that the two sheets were offset from one another, so that the individual graphene sheets could be addressed individually. SEM images of the final heterostructure can be seen in fig 6.12. In (a) the sample was tilted to an angle of $70^{\circ}$ whilst in the SEM chamber, to allow different the offset graphene sheets on the sample to be shown, as labelled. Both transferred graphene sheets show large perforations in the surface, which is a result of the water layer trapped underneath the graphene after transfer. 
This causes the graphene to be well-adhered in some places whilst not in others, with the resulting movement causing ripping in the sheet due to the addition of acetone (to remove PMMA). Fig 6.12b highlights the four different regions in the film of the heterostructure in more detail, easily discernible in the SEM images. To closely inspect the success of this transferred top graphene layer, highly magnified SEM images are shown in fig 6.13. This was important as due to the large height differences that have been pre-determined through AFM measurements, there was a cause for concern that the resulting large height changes for the graphene sheet could enhance small-scale ripping within the sheet as it spans the nanoparticle surface.

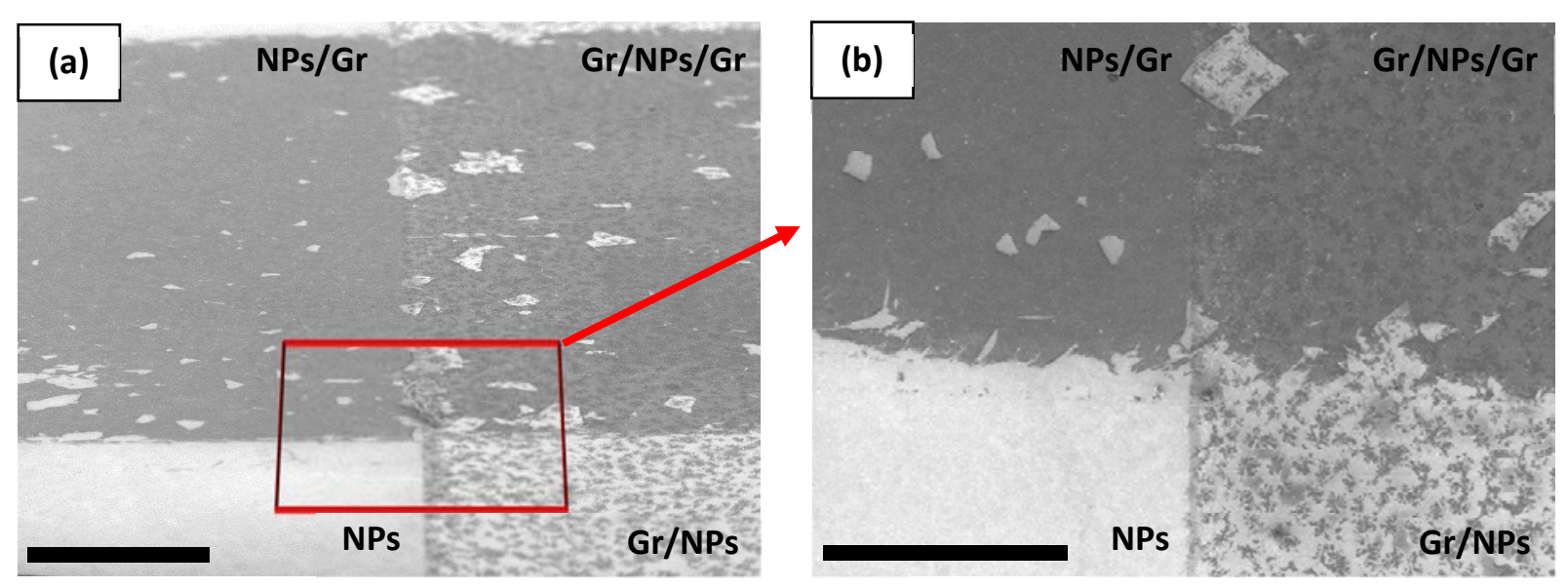

Figure 6.12: $\mathrm{SEM}$ images of a graphene/CQD/graphene heterostructure on $\mathrm{SiO}_{2}$, showing (a) a $70^{\circ}$ tilted image to inspect the overall sample and (b) a top view of the intersection of 4 separate regions. Different regions of the heterostructure are labelled in the figure. Scale bars $500 \mathrm{~nm}$.

As can be seen in the images, this top graphene sheet seems to conform to the nanoparticle layer extremely well, and no clearly visible tears were observed anywhere on the sample. The quality of the graphene transfer is further evidenced by the lack of rippling or undulations in the graphenegraphene regions at the edge of the deposited nanoparticle film.

An interesting observation was the directionality of the rippling of the graphene, which seems to follow a general trend, approximately horizontally across the image in this orientation. The mechanism behind this observation is thought to be a result of the dipping process, as the substrate is pulled vertically upwards through the nanoparticle film on the subphase. It is suggested that this 
results in a height variation in the nanoparticle film that is deposited in a direction that is approximately parallel to the subphase surface, due to the substrate being pulled out slowly and allowing more than a single monolayer to deposit in this preferential direction. These height fluctuations then correspond to the directionality of ripples in the top graphene layer. It should be noted that it was initially thought that this could be due to an artefact of the sample tilt during image capture, but by rotating the sample, the same directionality was observed.
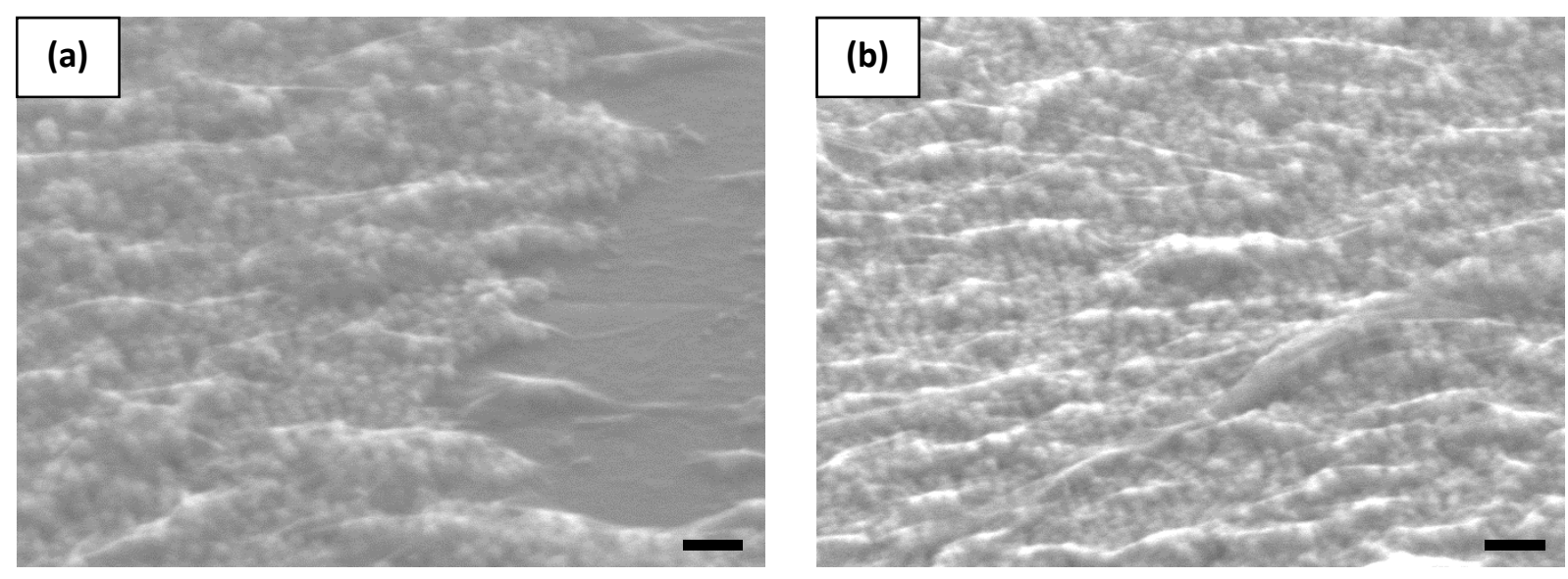

Figure 6.13: Highly magnified SEM images of the $\mathrm{Gr} / \mathrm{NP} / \mathrm{Gr}$ heterostructure, taken at $70^{\circ}$ sample tilt. This allowed any perforations in the top graphene layer to be investigated, and demonstrated a high quality conformation of the top graphene contact to the NP film, although an interesting directionality effect was observed. Scale bars are $100 \mathrm{~nm}$.

\subsubsection{Analysis of Graphene/NP/Graphene Heterostructures}

To investigate the mechanical and conducting properties of the fabricated heterostructure, a number of surface probe techniques were utilised simultaneously. The use of atomic force microscopy allowed the topography of the heterostructures to be investigated, whilst UFM and conductive AFM allowed the local mechanical and electrical conductive properties to be explored and correlated directly to the topographical image. The resolution of the images here is slightly reduced due to the need to use a diamond-tip, which are typically $\approx 100 \mathrm{~nm}$ in size. The addition of the top graphene contact provided some ability to the NPs which meant that contact mode could be again used. In these images, the drive amplitude was increased to $100 \mathrm{mV}$, scanning 512 samples per line with a rate of $0.9 \mathrm{~Hz}$. These values would have resulted in an extremely noisy image if not for the presence of this graphene layer. 
In fig 6.14, a region that spans the edge of a deposited nanoparticle film is shown in in the left-panel, this allows a direct comparison of the graphene/graphene interface and graphene/NP/graphene heterostructure to be explored. Whilst in the right-panel, an entire region comprising the heterostructure was imaged. As expected, the UFM images show the anticipated signal, where the graphene/graphene interface is extremely stiff as witnessed by the bright contrast in these regions (top-left area of left-panel). Taking the average of the collected signal in the regions with nanoparticles and no nanoparticles gave $-0.04 \pm 0.06 \mathrm{~V}$ and $2.17 \pm 0.06 \mathrm{~V}$, respectively. This gives an approximate contrast difference between the two areas of $55.3 \pm 4.8 \%$, corresponding to the heterostructure region being 'half as stiff' as the graphene/graphene region. This difference was anticipated as the height variations in the nanoparticle deposition observed in the SEM allows for movement of the nanoparticles when probed with the UFM, and it is anticipated that if the process is tuned to give a single monolayer deposition then this contrast should be vastly reduced. It was also observed that within the regions comprising nanoparticles, the stiffness showed lots of variation. This can also be explained through the height differences across the nanoparticle film, with higher regions of the film being less stiff, a result of the non-uniform stacking allowing the nanoparticles more movement when compressed with the AFM tip. It is expected that in the right-panel, the brightest contrast regions within the UFM measurement are regions where the graphene is dipping in between gaps in the nanoparticle film to touch the bottom graphene and becoming stiffer than the remainder of the film. However, the contrast difference here is difficult to obtain due to the large amount of fluctuation, and it was estimated to be $\sim 20 \%$ less than the value reported above. This discrepancy between values is believed to arise from the semi-contact of these dipped graphene layers, which would not be as stiff as a well-adhered graphene/graphene region; although the imprecision of the second result may also be able to account for this discrepancy. The small particles seen within the graphene layer is thought to arise from excess PMMA leftover after the transfer process. 
Gr/Gr (top-left) and Gr/NP/Gr (bottom-right) region
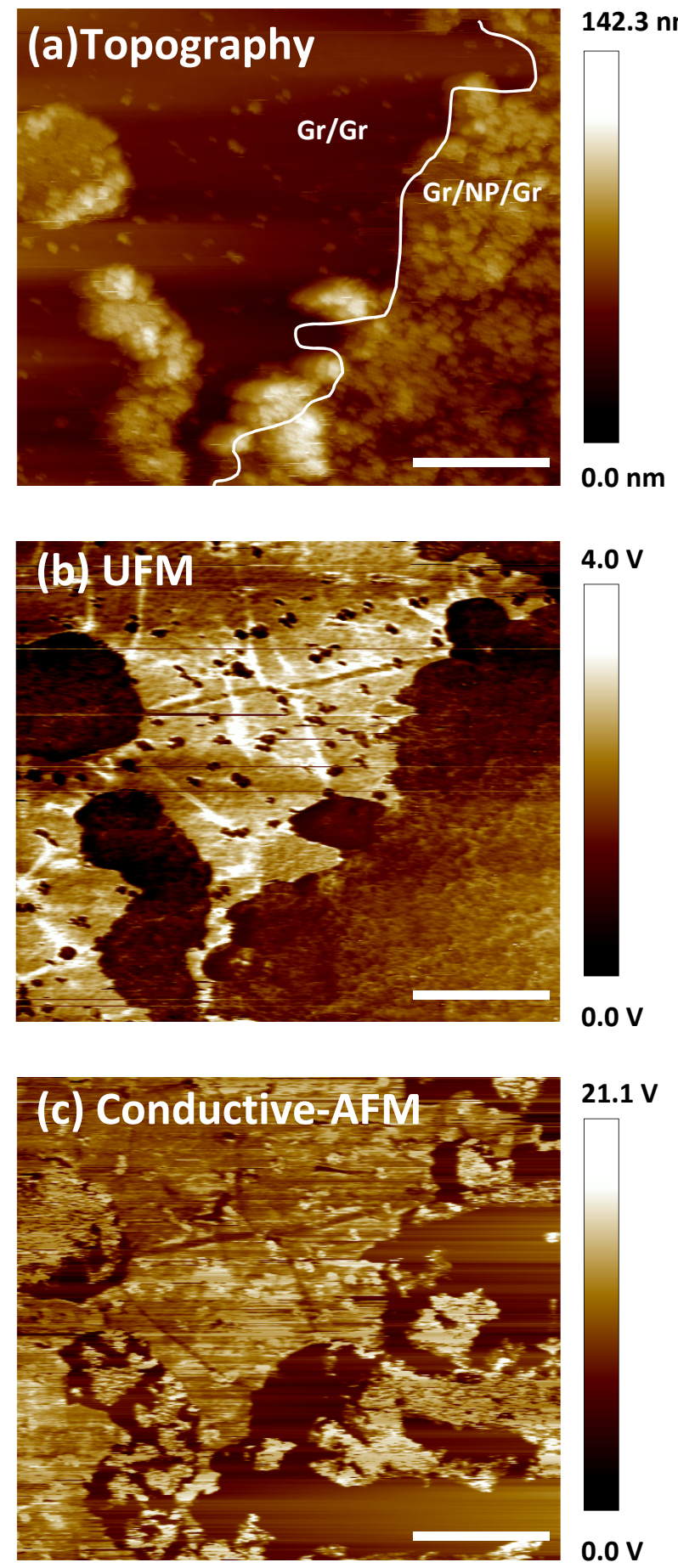
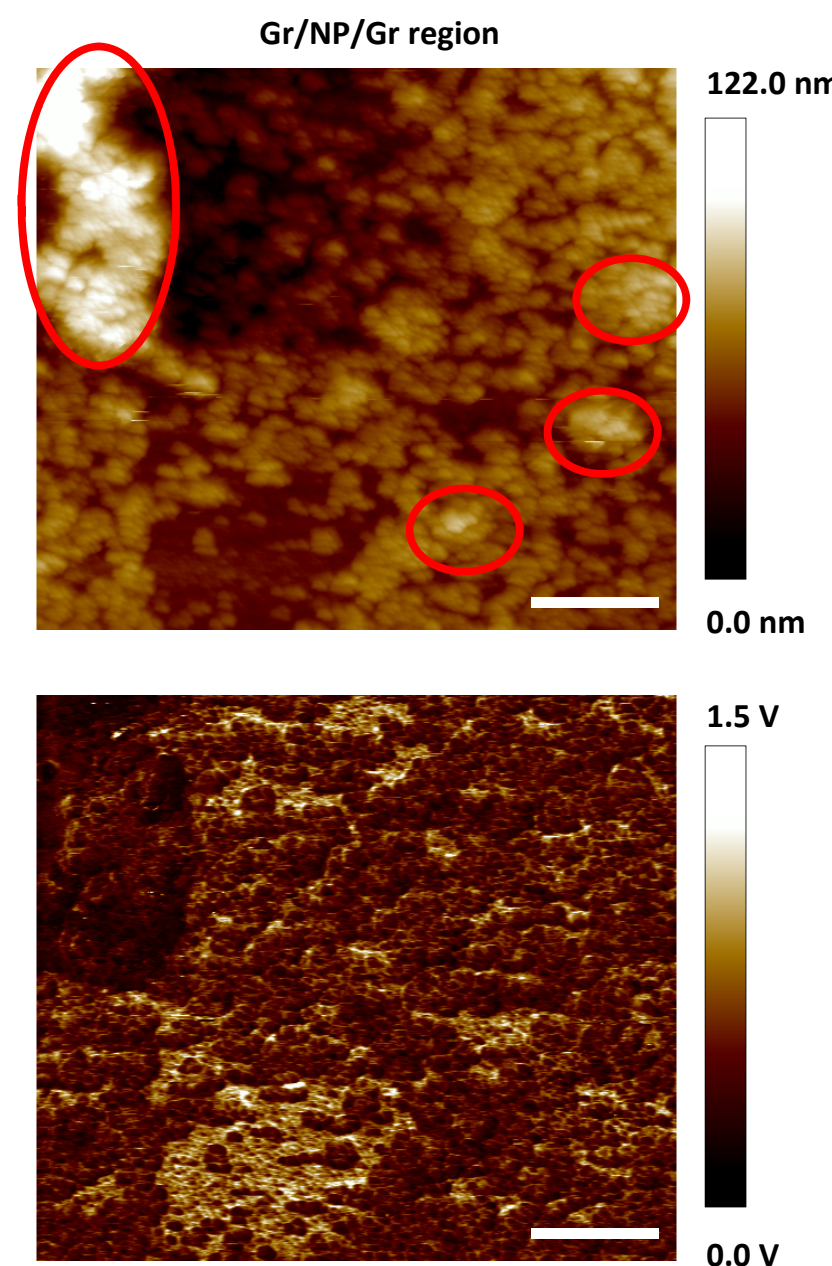

$1.5 \mathrm{~V}$
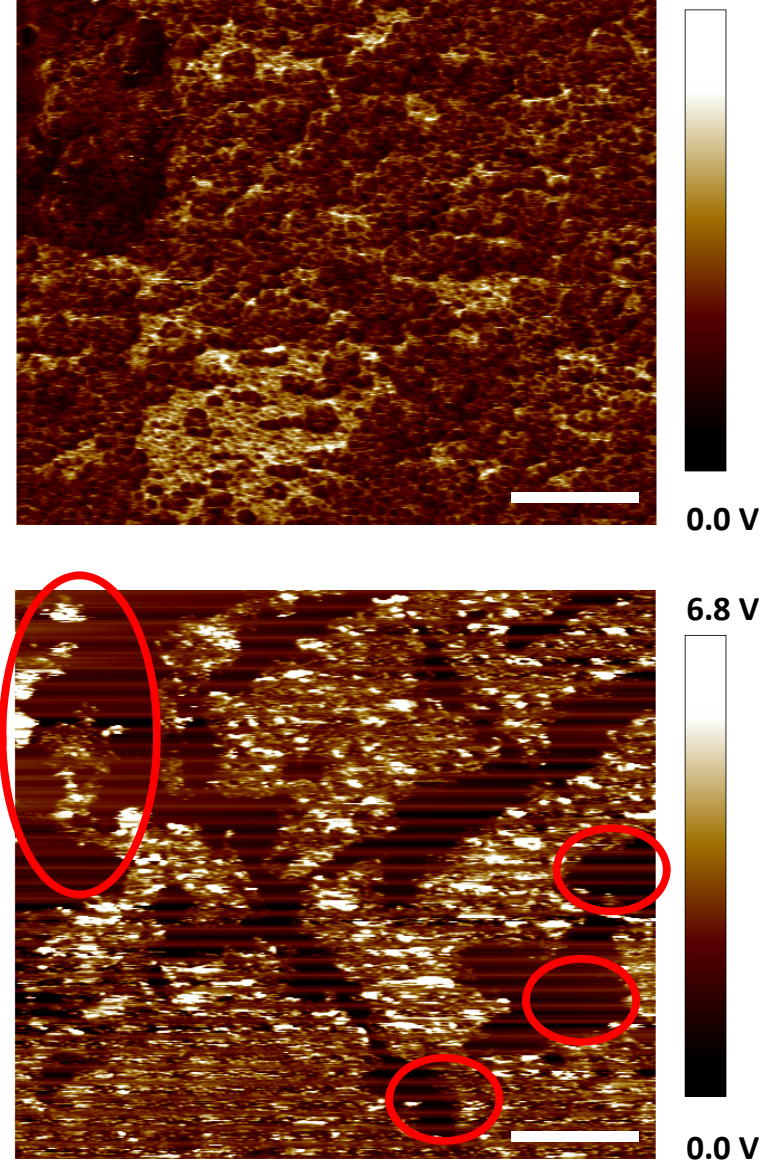

Figure 6.14: (a) Topography, (b) UFM and (c) Conductive AFM of Gr/NP/Gr heterostructures. Left panel shows a $\mathrm{Gr} / \mathrm{Gr}$ region next to the complete heterostructure to highlight the differences. The right panel shows just the Gr/NP/Gr heterostructure, to enable enhanced contrast. The UFM showed a reduction in contrast for the heterostructure region, compared to the $\mathrm{Gr} / \mathrm{Gr}$ region. The C-AFM images demonstrated a perplexing signal, with areas of high conductivity being observed almost randomly in the heterostructure region. The red highlighted circles show a relation between high regions of NPs and lower conductivity. All scale bars $1 \mu \mathrm{m}$. 
The conductive-AFM measurements are still not fully understood and measurements are ongoing to understand the conductivity through the heterostructure. In the regions where the graphene layers are connected, a bright signal corresponding to large currents is measured, as expected. However, when the nanoparticle film is present an insulating barrier between the two graphene sheets is expected, allowing current to flow only through tunneling ${ }^{20}$. By averaging a number of regions in the C-AFM image (left panel), the difference in measured voltage between graphene/graphene and graphene/NP/graphene was found to be $7.03 \pm 0.86 \mathrm{~V}$, corresponding to a difference in current of $70.3 \pm 8.6 \mu \mathrm{A}$. The measured voltage at a single point in the C-AFM map actually corresponds to the tip sampling an area similar to the size of the tip ( $100 \mathrm{~nm}$ diameter). By using the average voltage measured across a large area of the graphene/graphene region, these areal variations produced by the finite tip size are taken into account. Subsequently, by using the applied bias voltage of $6 \mathrm{mV}$ and the external resistance of $100,000 \Omega$, allows the resistance of the graphene/graphene to be calculated as $79.3 \pm 7.7 \Omega$, in agreement with previous out-of-plane measurements of bilayer graphene ${ }^{28}$. Whereas the resistance of the low-conducting regions in the nanoparticle film is $1132.1 \pm 277.7 \Omega$; where the larger percentage error comes from the large fluctuation of current in these regions. This value is still particularly low, as an insulating barrier like this should produce a resistance change of approximately three orders of magnitude ${ }^{20}$. Interestingly, the data collected here shows areas of low and high conductivity as different areas of the nanoparticle film is probed. The mechanism behind this is not fully understood, although there are a couple of possible explanations. First, the excess solvent/water leftover following deposition and transfer of the top graphene is thought to be trapped in between the graphene layers, this could create current conducting pathways resulting in areas of conductivity within the heterostructure where water is present. However, it has been observed that the presence of such leftover solvent and moisture act to reduce the vertical conductivity ${ }^{29}$.

In these measurements, the current has been observed to increase past the average graphene/graphene current in some regions, which suggests another mechanism than trapped water/solvent. Other processes that can reduce the out-of-plane conductivity include leftover 
polymer residue and air pockets ${ }^{29}$. However, it can be seen that the PMMA regions show negligible change in measured current whilst air pockets should have a higher probability of arising in the heterostructure region, so cannot explain the larger current values here. An alternative possibility is the conformation of the graphene to the nanoparticles being extremely tight, such that wherever there is the smallest gap in the film, the graphene layers contact one another (especially when force is applied by the AFM tip). This could create current carrying pathways even in regions where the graphene is above a nanoparticle, as the current could effectively 'flow around' to the bottom graphene contact. This could explain the local increases of measured current, where these areas have a simple current path as opposed to the overall average on the graphene/graphene region which takes into account the air/water/PMMA traps over a large area. There is some evidence for this in the CAFM in the right-panel, as the larger regions highlighted in the topography image seem to show some correlation with regions of lower conductivity. This would agree with the proposed scenario, as the graphene suspended over higher regions of the film would have to travel further to reach the bottom contact layer, thus showing lower conductivity. This is supported by the low resistance measurement stated above, which should be much higher if these regions were truly insulating. Finally, it is not expected that all the current would flow directly to the bottom graphene sheet in connected areas, as the sheet resistance of bilayer graphene has been found to be $287-288 \Omega \mathrm{sq}^{-1}$, so over larger length scales the path of least resistance would be through the $\mathrm{NPs}^{30,31}$.

\subsection{Light Output from Devices}

\subsubsection{Fluorescence from Heterostructures}

The nanoparticles used for the creation of heterostructures had a band gap corresponding to green light, at approximately $580 \mathrm{~nm}$. This can be seen in fig 6.15 which shows the fluorescence collected from the nanoparticles suspended in solution, and drop-cast onto an $\mathrm{SiO}_{2}$ substrate. It was observed that the drop-cast sample showed $a \approx 2.5 x$ increase in the fluorescent intensity. 


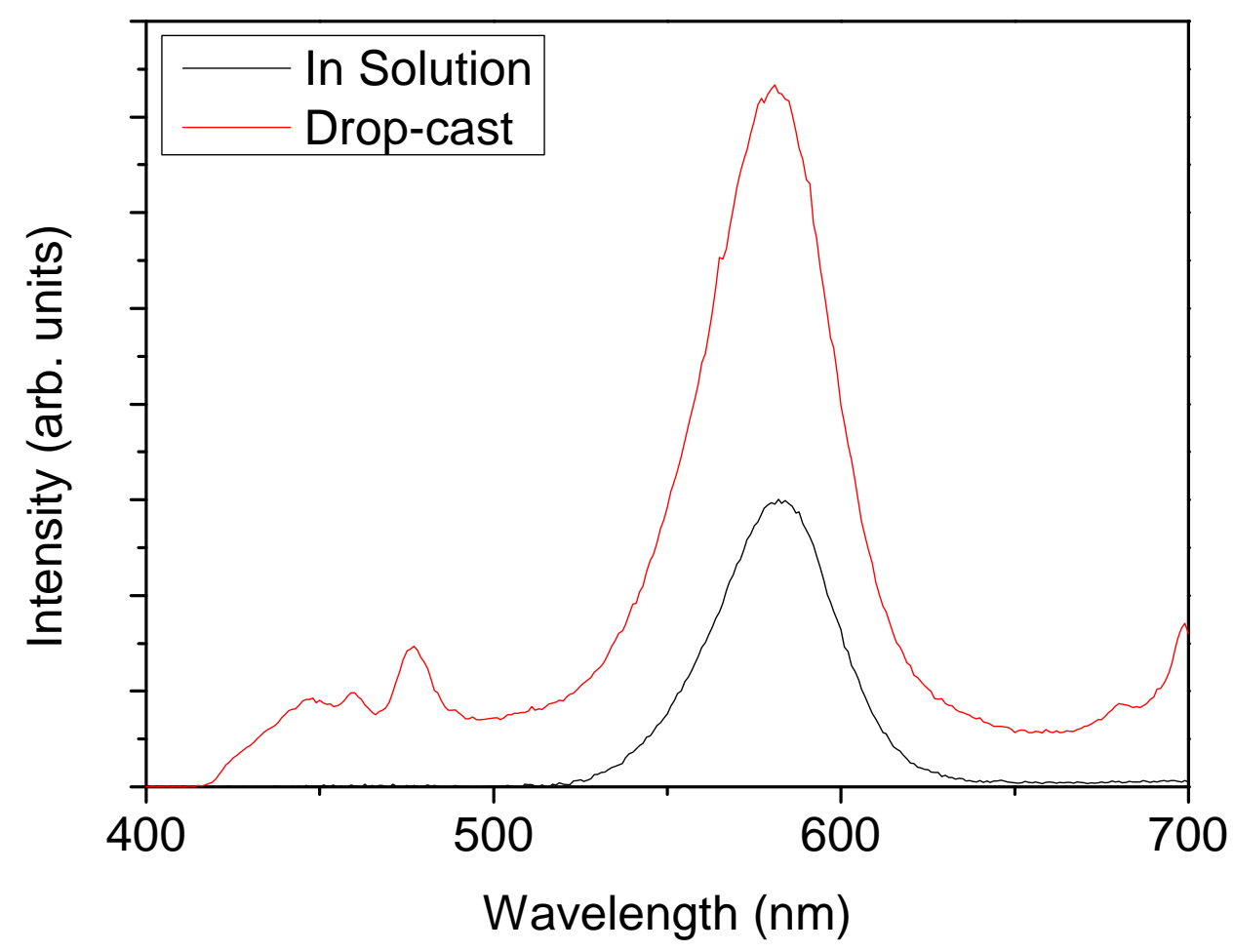

Figure 6.15: Fluorescence from CdSe-Si nanoparticles in ethanol solution (black) and drop-cast onto $\mathrm{SiO}_{2}$ (red). The drop-cast samples increased intensity is a result of reflection from the substrate and the high density of NPs once deposited.

This enhancement is thought to be due to both the reflection from the substrate as the NP emission is omnidirectional, and the high density of nanoparticles on the surface. Furthermore, the collection of signal at $450-500 \mathrm{~nm}$ and $700 \mathrm{~nm}$ is due to emission from the silicon substrate itself. However, when a fabricated heterostructure sample was investigated with the fluorescence spectrometer, the signal was completely quenched. A number of possible reasons were suggested to explain this, such as: charging of nanoparticles through the electrospray, interaction with the water subphase, a graphenenanoparticle interaction, and thin nanoparticle layers providing limited intensity. Through creation of a number of samples, each of these mechanisms were investigated as the reason for quenching and it was concluded that the nanoparticles suffered from the interaction with the water subphase over a long period of time. The nanoparticles continue to fluoresce if only in contact with the water for a brief period of time (5 mins), but quench when left over a number of hours (as required by the electrospray, Langmuir-Blodgett process). The process causing this quenching is thought to be the 
capture of free radicals within the water, such as $-\mathrm{OH}$ acceptors ${ }^{32}$, which create traps for charge carriers and prevent radiative recombination.

\subsubsection{Changing the Subphase}

Following the application in previous reports, the nanoparticles were deposited using the chemical dimethyl sulfoxide (DMSO) as the subphase ${ }^{27}$. However, in this case, the subphase and spreading solvent were still miscible so electrospray spreading was still employed. Moreover, the use of less polar subphases allow better spreading, whilst freeing up the entire surface for nanoparticle film formation ${ }^{27}$. In particular, it is hypothesised that the lower polarity results in free, unattached ligands present in the solution to dissolve into the DMSO, leaving the surface completely available for NP film formation. In fig 6.16, an optical and SEM image of the deposited nanoparticle film can be seen.

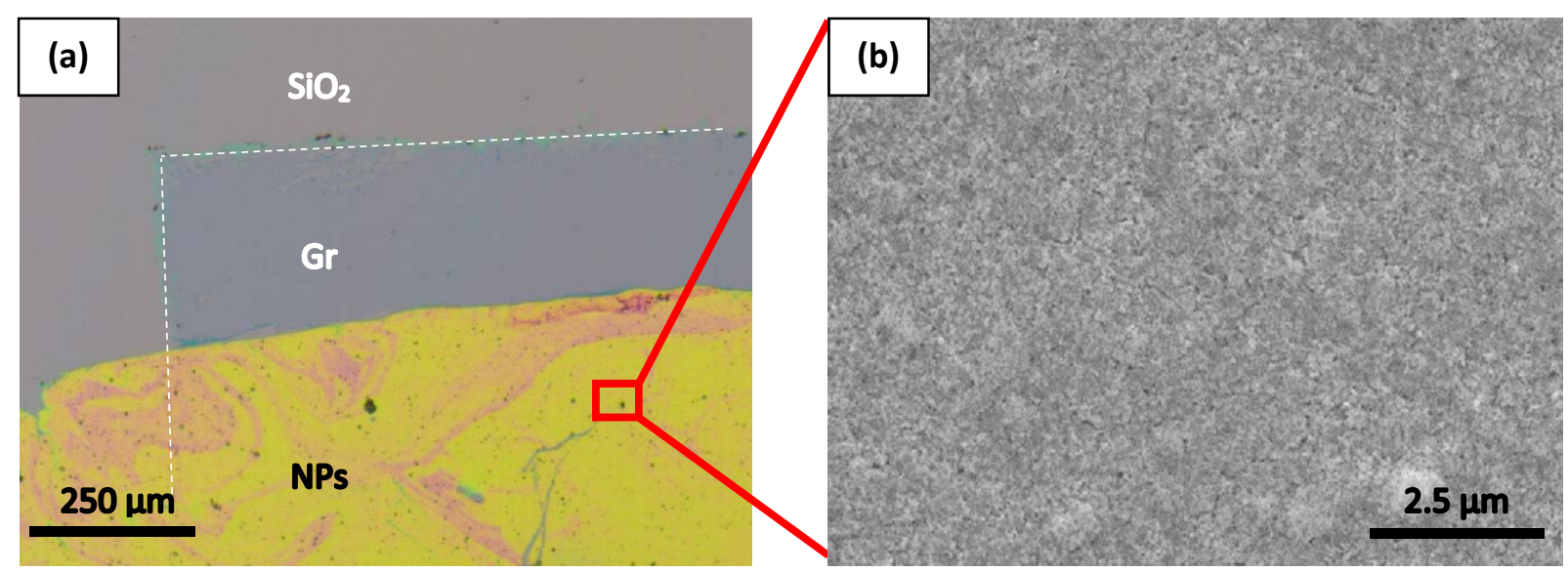

Figure 6.16: Preparation of a NP film through the use of a DMSO subphase, showing (a) an optical image which highlights the $\mathrm{SiO}_{2}$, graphene and NP regions. The NPs, like before, show uniform coverage over both regions. The graphene edge is highlighted. (b) SEM image of the region located on the graphene, showing a much higher packing with no visible gaps in the film.

It was observed immediately in the optical image (fig 6.16a) that the existence of gaps in the nanoparticle film are vastly reduced, as opposed to the films created from an air-water interface (see fig 6.5a).

As was observed for the deposition from the air-water interface, it can again clearly be seen that the nanoparticles have formed on both $\mathrm{SiO}_{2}$ and graphene with negligible differences. To investigate this 
film, the SEM was employed and a representative image is shown in fig $6.16 \mathrm{~b}$, the close-packing of the nanoparticles in the film is readily apparent.

The gaps that were witnessed previously seem to have been reduced, indicating that the nanoparticles spread much more efficiently on the DMSO than on the water. Furthermore, the area on the SEM is large enough to form a $5 \times 5 \mu \mathrm{m}^{2}$ device and should prevent graphene/graphene short circuits due to sagging between gaps in the nanoparticles.

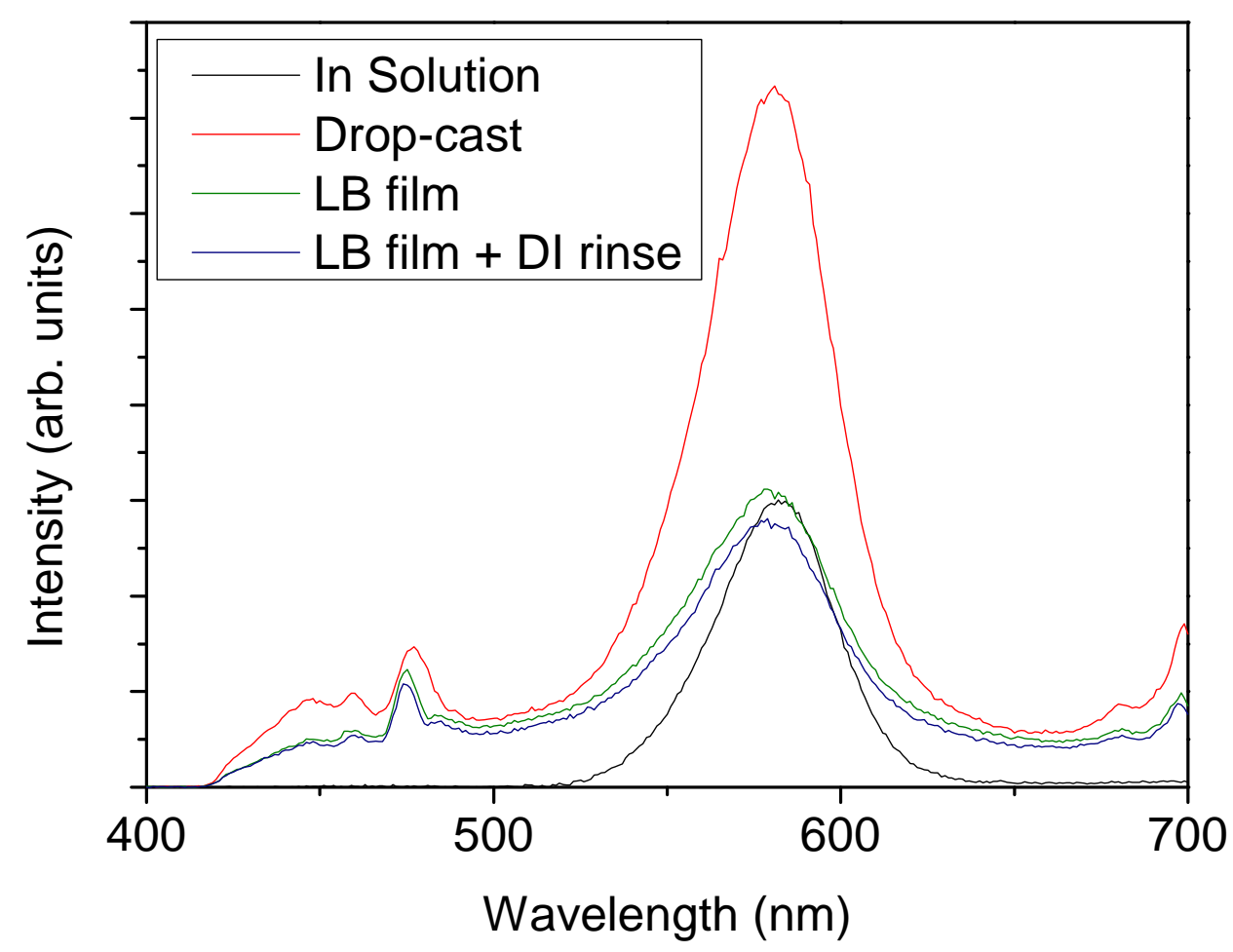

Figure 6.17: Comparison of fluorescence of LB-deposited NP films, drop-cast NPs and NPs in solution. Through the use of DMSO as the subphase, the fluorescent intensity remains high. Furthermore, exposure to water for 5 minutes does not quench the fluorescence, a key requirement for transfer of the top graphene contact.

To elucidate whether the nanoparticle film still emits following deposition from DMSO, fluorescence spectroscopy was again employed. The data for this is shown in fig 6.17 , it was observed that the fluorescent signal is at the same order of magnitude as that measured in solution, evidencing that the quenching has been removed, or at least reduced. The intensity is much less than the drop-cast sample, suggesting that the high intensity observed in that case was principally due to the high density 
of nanoparticles rather than reflection from the substrate. To test the effect of water on the deposited film, the sample was rinsed in deionised water for 5 minutes. This was necessary as the creation of double heterostructures requires the sample to be placed in an aqueous environment for approximately this period of time. Advantageously, the nanoparticles still emit at approximately the same level of intensity (although there is a slight reduction), providing evidence that the quenching effect in the case of water is due to the long time period between deposition and transfer.

Interestingly, a small blue-shift and peak broadening has been observed in the deposited film which is thought to arise due to dense packing of the nanoparticles and intermolecular interactions, respectively ${ }^{33}$. Using this subphase should allow for the creation of graphene/NP/graphene heterostructures that contain a truly insulating barrier, whilst emitting bright visible fluorescence, and will be the subject of future work.

\subsection{Summary}

In the work presented here, heterostructures combining two-dimensional and zero-dimensional quantum systems have been fabricated by the Langmuir-Blodgett technique, for the first time. In particular, CdSe nanoparticles with silica coatings have been used, allowing non-toxic chemicals to be used for suspension and offering a much greater integration potential. This facile method could allow the scalable creation of optoelectronic devices capable of emitting at a range of wavelengths to be produced, and large steps have been taken in this work to achieve this goal.

It has been shown that through the application of electrospray for the spreading of miscible liquids, nanoparticle films could be created at the air-water interface and deposited onto $\mathrm{SiO}_{2}$ and graphene, with coverage percentages of $63.55 \pm 3.20 \%$ and $64.83 \pm 2.75 \%$, respectively (for a given type of NP). Through study of the nanoparticle films using scanning electron microscopy, an average value for the diameter of nanoparticles used for creation of heterostructures was found to be $30.5 \pm 0.3 \mathrm{~nm}$, which agrees with the estimated thickness of the nanoparticle given the core and shell sizes; the order of this film was also observed to be uniform but highly discontinuous. Furthermore, the use of atomic 
force microscopy allowed the thickness of the deposited films to be determined and it was found that the heights varied from 1-6 layers of nanoparticles. The average height of a film was calculated as 38.4 $\pm 2.4 \mathrm{~nm}$, indicating a double layer of nanoparticles has been deposited on average.

Graphene/NP/graphene heterostructures were created using a wet transfer process and ultrasonic and conductive force microscopy was applied to investigate their properties. It was found that the stiffness of the heterostructure region reduced by $55.3 \pm 4.8 \%$, compared to the graphene/graphene region, indicating that the nanoparticles were not tightly packed under the top graphene layer. Furthermore, the conductivity measurements yielded a resistance for the graphene/graphene and graphene/NP/graphene regions of $79.3 \pm 7.7 \Omega$ and $1132.1 \pm 277.7 \Omega$, respectively. These values inherently include the average over a local area of the film, dictated by the size of the probe tip. The first value agreed with previous reports for bilayer graphene but the heterostructure region should display an increase by three orders of magnitude. Thus, it was concluded that the graphene was dipping in between nanoparticles to create short-circuits, which needs to be remedied before efficient optoelectronic structures can be made.

The fluorescence measurements showed that the creation of a Langmuir-Blodgett film at the air-water interface seemed to quench the emission, as compared to the emission from nanoparticles in solution and drop-cast onto an $\mathrm{SiO}_{2}$ substrate. This was investigated and concluded as being due to the large period of time in which the nanoparticles were present on the water surface. A dimethyl sulfoxide subphase was then employed to deposit nanoparticles and it was shown that the fluorescence signal has not been quenched whilst the created film is much more close-packed.

In future work, the creation of Gr/NP/Gr heterostructures deposited from dimethyl sulfoxide will be created. Their conductivity/mechanical properties will be explored using alike methods and compared to the previously studied samples. Furthermore, upon the fabrication of successful heterostructures without short-circuits, individual devices will be isolated and electrical contacts defined. This will allow the characterisation via transport measurements and electroluminescence measurements. 


\section{Bibliography}

${ }^{1}$ Z. Alfassi et al., "Photochemistry of Colloidal Metal Sulfides. 3. Photoelectron Emission from Cadmium Sulfide and Cadmium Sulfide-Zinc Sulfide Cocolloids", J. Phys. Chem., 86, 24 (1982).

${ }^{2}$ A. L. Efros et al., "Interband Absorption of Light in a Semiconductor Sphere", Sov. Phys. Semicond., 16, 7 (1982).

${ }^{3}$ L. E. Brus, "Electron-electron and Electron-hole Interactions in Small Semiconductor Crystallites: The Size Dependence of the Lowest Excited Electronic State", J. Chem. Phys., 80, 4403 (1984).

${ }^{4}$ Y. Yin et al., "Colloidal Nanocrystal Synthesis and the Organic-inorganic Interface", Nature, 437, 664 (2005).

${ }^{5}$ D. V. Talapin et al., "Seeded Growth of Highly Luminescent CdSe/CdS Nanoheterostructures with Rod and Tetrapod Morphologies", Nano. Lett., 7, 10 (2007).

${ }^{6}$ S. Doose, “Optical Amplification from Single Excitons in colloidal Quantum Dots”, Small, 3, 1856 (2007).

${ }^{7}$ D. Schooss et al., "Quantum-dot Quantum Well CdS/HgS/CdS: Theory and Experiment", Phys. Rev. B., 49, 17072 (1994).

8 J. M. Pietryga et al., "Utilizing the Lability of Lead Selenide to Produce Heterostructured Nanocrystals with Bright, Stable Infrared Emission", J. Am. Chem. Soc., 130, 14 (2008).

${ }^{9}$ K. L. Janssens et al., "Single and Vertically Coupled Type-II Quantum Dots in a Perpendicular Magnetic Field: Exciton Ground-state Properties", Phys. Rev. B., 66, 075314 (2002).

${ }^{10}$ V. I. Klimov et al., "Optical Gain and Stimulated Emission in Nanocrystal Quantum Dots", Science, 290, 5490 (2000).

${ }^{11}$ S. T. Selvan et al., "Synthesis of Tunable, Highly Luminescent QD-glasses Through Sol-gel Processing", Adv. Mater., 13, 985 (2001).

${ }^{12}$ C. B. Murray et al., "Synthesis and Characterisation of Nearly Monodisperse CdE (E= Sulfur, Selenium, Tellerium) Semiconductor Crystals", J. Am. Chem. Soc., 115, 19 (1993).

${ }^{13}$ M. A. Hines et al., "Synthesis and Characterisation of Strongly Luminescing ZnS-capped CdSe Nanocrystals", J. Phys. Chem., 100, 2 (1996).

${ }^{14}$ L. Qu et al., "Control of Photoluminescence Properties of CdSe Nanocrystals in Growth", J. Am. Chem. Soc., 124, 9 (2002).

${ }^{15}$ T. Pellegrino et al., "Hydrophobic Nanocrystals Coated with an Amphiphilic Polymer Shell: A General Route to Water Soluble Nanocrystals", Nano. Lett., 4, 4 (2004).

${ }^{16}$ B. Dubertret et al., "In Vivo Imaging of Quantum Dots Encapsulated in Phospholipid Micelles", Science, 298, 5599 (2002).

${ }^{17}$ W. C. W. Chan et al., "Quantum Dot Bioconjugates for Ultrasensitive Nonisotopic Detection", Science, 281, 5385 (1998).

18 P. Mulvaney et al., "Silica Encapsulation of Quantum Dots and Metal Clusters", J. Mater. Chem., 10, 1259 (2000).

${ }^{19}$ C. Graf et al., "Metallodielectric Colloidal Core-shell Particles for Photonic Applications”, Langmuir, 18, 2 (2002). 
${ }^{20}$ C. Lu et al., "Electronic Transport in Nanoparticle Monolayers Sandwiched Between Graphene Electrodes", Nanoscale, 6, 14158 (2014).

${ }^{21}$ M. Acebrón et al., "Protective Ligand Shells for Luminescent $\mathrm{SiO}_{2}$-coated Alloyed Semiconductor Nanocrystals", ACS Appl. Meter. Interfaces, 7, 6935 (2015).

${ }^{22}$ G. Mao et al., "Oleic Acid Disorders Stratum Corneum Lipids in Langmuir Monolayers", Langmuir, 29, 4857 (2013).

${ }^{23}$ X. Li et al., "Large-area Synthesis of High-quality and Uniform Graphene Films on Copper Foils", Science, 324, 5932 (2009).

${ }^{24}$ X. Li et al., "Evolution of Graphene Growth on Ni and Cu by Carbon Isotope Labelling", Nano. Lett., 9, 12 (2009).

${ }^{25}$ S. M. Kim et al., "The Effect of Copper Pre-cleaning on Graphene Synthesis", Nanotechnology, 24, 365602 (2013).

${ }^{26}$ www.microchem.com

${ }^{27}$ V. Aleksandrovic et al., "Preparation and Electrical Properties of Cobalt-Platinum Nanoparticle Monolayers Deposited by the Langmuir-Blodgett Technique", ACS Nano, 2, 6 (2008).

${ }^{28}$ J. Chen et al., "Layer-by-layer Assembly of Vertically Conducting Graphene Devices", Nat. Comms., 4, 1921 (2013).

${ }^{29}$ Y. Liu et al., "Giant Enhancement in Vertical Conductivity of Stacked CVD Graphene Sheets by Self-assembled Molecular Layers", Nat. Comms., 5, 5461 (2014).

${ }^{30}$ M. J. Madito et al., "Raman Analysis of Bilayer Graphene Film Prepared on Commercial Cu (0.5 at\% Ni) Foil", J. Raman. Spectrosc., 47, 5 (2015).

${ }^{31} \mathrm{~S}$. Chen et al., "Synthesis and Characterisation of Large-area Graphene and Graphite Silms on Commercial Cu-Ni Alloy Foils", Nano. Lett., 11, 3519 (2011).

${ }^{32} \mathrm{E}$. M. Samsonova et al., "Fluorescence Quenching Mechanism for Water-dispersible $\mathrm{Nd}^{3+}: \mathrm{KYF}_{4}$ Nanoparticles Synthesized by Microwave-hydrothermal Technique", Journal of Luminescence, 169, 722 (2016).

${ }^{33}$ L. Skardžiūtè et al., "Optical Study of the Formation of Pyrrolo[2,3-d]pyrimidine-based Fluorescent Nanoaggregates", Tetrahedron, 69, 9566 (2013). 


\section{Chapter 7}

\section{Conclusions and Future Work}

\subsection{Conclusions}

In this thesis, three example unique identification devices based on the atomically imperfect structure of their corresponding semiconductor systems have been created for the first time. Semiconducting systems displaying the appropriate properties for electrical, optical and optoelectronic measurements of identity have been specially picked and studied. Electronic measurements of identity were achieved using Resonant Tunneling Diodes (RTDs), where the imperfections in the quantum well results in fluctuations in the current-voltage spectra, which was used to identify the device. The twodimensional material $\mathrm{MoS}_{2}$ was chosen as the convenient optical device. A major bottleneck to the scalability of $2 \mathrm{D}$ materials for practical applications is the serial nature of standard fabrication methods. Here, the Langmuir-Blodgett technique was employed to create macroscopic thin films of $\mathrm{MoS}_{2}$, for the first time. The optoelectronic device architecture consisted of a graphene/nanoparticle/graphene heterostructure, where the nanoparticles were deposited using the Langmuir-Blodgett technique to create large-area heterostructures with silica capped nanoparticles, for the first time.

The electronic measurement of an RTD results in a spectrum containing a peak. This peak corresponds to a resonance with the confined energy level contained within the quantum well, this energy level is extremely sensitive to structural and compositional defects and imperfections in the well and the barriers. It was suggested in this work to use this peak as a definition of identity, for the first time. To provide a robust measurement of identity, the measurement parameters of the RTDs first had to be 
optimised such that repeated readings gave accurate results. Once the optimum parameters were found, the selection of available sizes of RTDs were explored to enable determination of the RTD geometry with the largest robustness; this was found to be the smallest RTD, containing a $2 \times 2 \mu \mathrm{m}^{2}$ mesa. Using the defined conditions and these RTDs, a total of 26 identical devices were measured electronically in exactly the same way. It was found that the peak positions of each of these devices lay in a unique location, after averaging 100 spectra to improve precision. Inspection of the closest lying peak positions elucidated that the peak positions do not overlap with a $99.997 \%$ precision, indicating the high degree of uniqueness in these devices. The 100 individual spectra from each device was compared to the average value and it was found that the individual peak positions were always within 2 standard errors of the average, demonstrating a high calibre of robustness in these devices. Although temperature will play a role in the resulting properties of the current-voltage characteristic, what is demonstrated here is a proof-of-principle and it is anticipated that such effects can be calibrated to add a further element of security to the device. Due to the unique, repeatable measurement, it was suggested that these devices could be used for unique identification, and would be incredibly difficult to clone whilst containing a secure bit density of $2.5 \mathrm{bits} / \mu \mathrm{m}^{2}$, bettering the state-of-the-art by a factor of two.

To create an optical analogue of a unique identification device, the use of optically active twodimensional materials was pursued. By inspecting individual monolayers, it was found that the emission spectra across a given flake varied measurably. The inefficiency of using single flakes lies in their need to be individually isolated and characterised each time, so the work presented here focussed on the fabrication of macroscopic thin films of 2D materials, namely $\mathrm{MoS}_{2}$. To this end, a facile method known as the Langmuir-Blodgett technique was used to create thin films of $\mathrm{MoS}_{2}$ for the first time. To achieve this, electrospray mediated spreading of solution was employed to minimise mixing of miscible solvents at the air-water interface. The presence of a thin film of $\mathrm{MoS}_{2}$ on the water surface was confirmed by pressure-area isotherms and Brewster angle microscopy. These films were then successfully transferred onto solid substrates to allow characterisation of their emission across 
the film. It was found that the photoluminescence from the $\mathrm{MoS}_{2}$ flakes had been quenched, and the mechanism behind this was explored to ensure it was not the Langmuir-Blodgett deposition that was the fundamental limitation. Scanning electron microscopy, atomic and ultrasonic force microscopy and Raman spectroscopy techniques were employed to probe the nature of the film. It was found that the film had an average thickness of $7.6 \pm 0.6 \mathrm{~nm}$, representing $\approx 12$ atomic layers. This was later corrected to $\approx 8$ layers as the apparent height of a monolayer was larger than that of a typical mechanically exfoliated monolayer. Thus, the lack of emission was attributed to the low monolayer content of the starting solution. To circumnavigate this problem, a new source was used containing 75\% monolayer content and preliminary results have shown Langmuir-Blodgett deposition of a thin film that displays large-area emission.

To complete the complimentary set of devices that can provide unique identification, an optoelectronic device was fabricated that based its design on graphene and semiconducting nanoparticles (NPs). The work presented here used the aforementioned Langmuir-Blodgett technique to build novel heterostructures combining these $2 \mathrm{D}$ and $\mathrm{OD}$ quantum systems; this $2 \mathrm{D}-\mathrm{OD}$ interface should readily enhance the uniqueness of such devices. The fabrication of graphene/NP/graphene heterostructures using silica-capped NPs, an advantageous non-toxic system, was demonstrated in this work for the first time. Here, the electrospray technique was again employed and it was found that deposited NP films had coverage percentages of $63.55 \pm 3.20 \%$ and $64.83 \pm 2.75 \%$ on $\mathrm{SiO}_{2}$ and graphene, respectively. The use of scanning electron microscopy allowed the diameter of various nanoparticles to be determined, and was $30.5 \pm 0.3 \mathrm{~nm}$ for the NPs used to create heterostructures. This was found to be in excellent agreement with the estimation of the core and shell sizes. The order and topography of the film was explored with atomic force microscopy and a mean height of $38.4 \pm$ $2.4 \mathrm{~nm}$ was found for the films, corresponding to a double layer of nanoparticles being deposited on average. To finalise the heterostructure, top graphene contacts were defined and ultrasonic (UFM) and conductive force microscopy (C-AFM) was employed to measure the mechanical and electrical properties of the heterostructure. It was found that the NPs were not well ordered when deposited 
and displayed a low stiffness when probed with the UFM. The resistance was measured as $79.3 \pm 7.7$ $\Omega$ and $1132.1 \pm 277.7 \Omega$ for the $\mathrm{Gr} / \mathrm{Gr}$ and $\mathrm{Gr} / \mathrm{NP} / \mathrm{Gr}$ regions, respectively. The former value agreed well with the out-of-plane resistance for bilayer graphene, but the heterostructure's resistance should have increased by three orders of magnitude if a completely insulating layer was formed. This indicated the presence of short circuits, where the graphene contacts have warped around the NPs to provide current allowing pathways. Finally, it was found that the fluorescent signal of the NPs in the heterostructure had diminished, so an alternative subphase, dimethyl sulfoxide, was employed. This remedied the fluorescence, whilst creating NP layers with a much larger packing on the surface; this should enable electroluminescent devices to be created.

\subsection{Future Work}

The measurements displayed in this thesis have involved the creation of semiconductor systems that would allow their atomic-scale uniqueness to be probed through measurements of macroscopic features. To this end, three device architectures have been suggested that would allow a measurement of identity electronically, optically, and optoelectronically. To increase the security and practicality of such devices, a number of methods can be explored for each system and will be discussed in this section.

\section{(a) Resonant Tunneling Diodes}

It has been shown that unique identities can be derived from RTDs. To increase the security of such a system requires the incorporation of more unique information within the device, and can be achieved by imposing a larger number of features into the device's I-V characteristic. In this work, a standard double-barrier quantum well structure was used, which displays a single peak relating to the confined state. Alternatively, the production of a device with multiple peaks can be achieved in a variety of ways, where each added peak would increase the secure information stored in the device. The first method to achieve this involves the direct linking of RTDs in circuitry, by connecting more than 1 RTD in series or in parallel, for example. This can result in an I-V spectrum that displays an equal number 
of peaks to the number of devices that have been combined in the circuit. This has been confirmed by preliminary studies, where the spectra in fig 7.1a shows the result obtained from connecting three RTDs in series.

Another method of increasing the number of unique features requires the use of nanostructures exhibiting higher orders of quantum confinement. If an RTD was fabricated with quantum dots in the active region, the number of unique peaks should increase dramatically. For example, the microphotoluminescence spectra shown in fig $7.1 \mathrm{~b}$ was extracted by exciting a $\sim 1 \mu \mathrm{m}^{2}$ area of a quantum dot sample, where the large number of unique peaks arises from a large density of quantum dots of different sizes in the sample. If these were used in an RTD with an area of $2 \times 2 \mu \mathrm{m}^{2}$, these unique peaks corresponding to different quantum dots should appear in the I-V and enable the electronic measurement of a large range of peaks which would vastly increase the security.
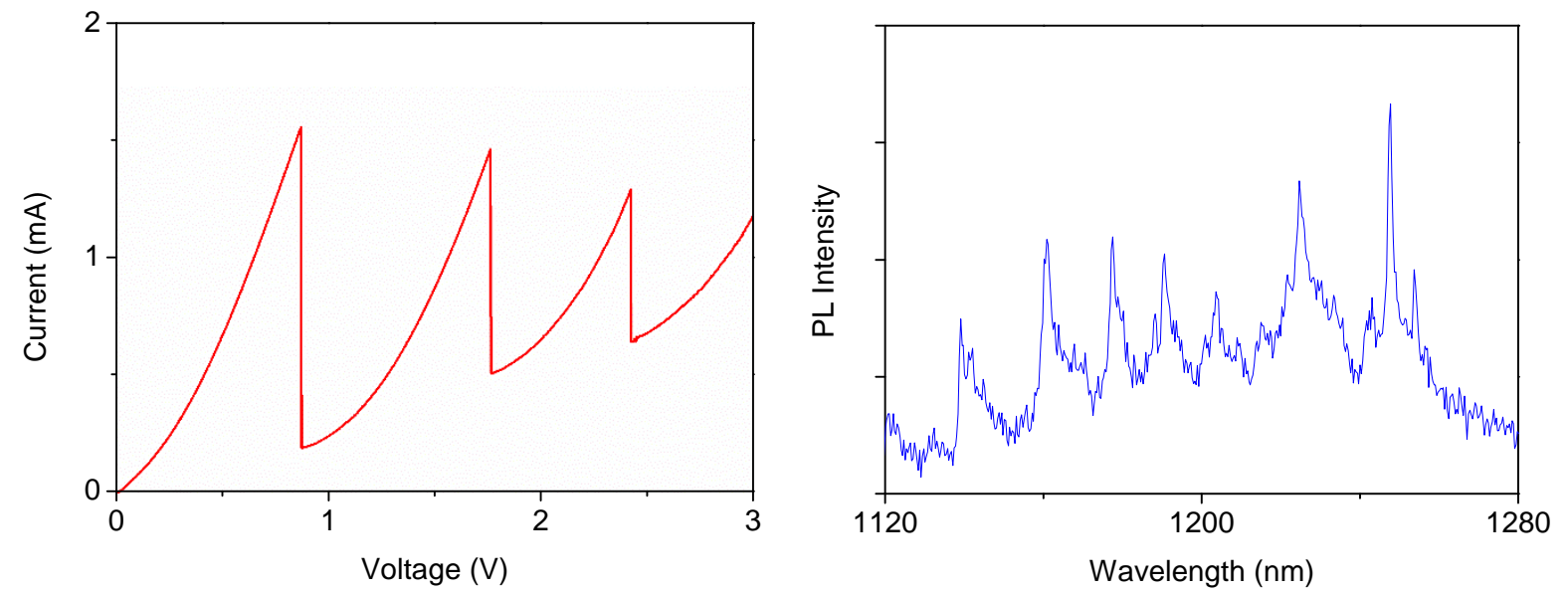

Figure 7.1: Increasing the security of RTD-based devices using methods to increase the number of features. (a) 3 RTDs connected in series in a circuit, showing three corresponding unique peaks, (b) spectrum of a quantum dot sample that should display these features in an RTD, if incorporated.

Another method of interest that could enable maximum information extraction includes using the other features present in a typical I-V characteristic. These include, the full-width half-maximum of the peak, the position of the valley, the peak-to-valley ratio and the value of all the above features in the opposing bias direction, although it is also required for these features to be robust. 
To increase the fluctuations of the well width and enhance the effect of imperfections in the measurement, it should be simple to deliberately add randomness to the RTD during the manufacture of its nanostructure. For example, by interrupting an epitaxial growth step during the formation of the double-barrier quantum well structure, much larger interfacial fluctuations would be observed which are normally undesirable, but would provide great advantages for this application. This explicit randomness could be added in all areas of the RTD, including the doping profiles.

It is also of interest to increase the practicality of such devices to allow incorporation into embedded systems. Obvious conditions such as the local temperature and humidity could affect the operation of the RTD, and it is important to discover whether the variations of the I-V due to these effects can be calibrated. These conditions could vary considerably if the device was present on an electronic circuit board, for example.

For practical implementations, the device needs to be robust over large time periods, so the longevity of RTDs needs to be explored. The exposure to the environment could enhance degradation over large time scales and encapsulation of RTDs in a non-conducting coating could be useful here.

A probe station is not the most practical setup for realistic implementation and was used here for simplicity. Thus, it is a requirement to embed RTDs into a chip-like architecture and add some external circuitry that can automatically measure the RTDs resonance. If anything, these results should display an improved robustness due to the direct bonding of devices, as opposed to probes which can cause intermittent contacts. Preliminary studies have begun here with the spectrum in fig 7.1 arising from the measurement of a bonded RTD within a custom circuit.

An interesting condition that can be explored is the controlled application of heat or current to a device. If the atomic-structure can be purposely changed without destroying the RTD, then this should provide a good way of 'resetting' the device if the unique fingerprint is discovered. Finally, before a true implementation, an extensive and robust security analysis must be carried out on a large array of devices. 


\section{(b) Macroscopic Deposition of $\mathrm{MoS}_{2}$}

It has been shown in this work that the successful fabrication of macroscopic thin films of $\mathrm{MoS}_{2}$ can be achieved via the Langmuir-Blodgett technique. By careful control of the properties of the starting solution, these thin films can be fabricated such that they emit over the entire surface. Now this is possible, to understand the usability of these thin films as individual devices providing unique identification of an object, the emission spectra needs to be explored. Specifically, using a large number of devices, complex optical maps of the films need to be taken to highlight the variations in emission. For these maps, the correlation of device dependent properties shall be explored to understand the uniqueness of such a system. This can be done through standard image processing techniques. The individual tags also need to provide a robust measurement of their emission map, therefore each map will be measured a number of times to ensure repeatability of the unique fingerprint.

The complexity of these systems can enhance the security of the system by creating a larger barrier to cloning. This can be achieved through creation of layered films with the Langmuir-Blodgett technique. By utilising films that emit at different wavelengths (using different materials), the optical emission of a device should be able to be expanded over the entire visible range. This would also assist in increasing the practicality of such a device, as the emission profile needs to be measured efficiently, so spreading the signal over the visible range would provide some scope for this to be achieved.

Although the initially deposited films displayed here showed film thicknesses corresponding to a number of layers, this could be used as an advantage. For example, the Langmuir-Blodgett films could be utilised in other applications which require the existence of a macroscopic film of material. An alternative suggestion is to utilise two-dimensional materials that emit light due to quantum confinement when there layer number is greater than 1 . An example of this type of system would be InSe. By creating large-area films of InSe, the strict requirements of monolayer deposition would be removed and would relieve some of the difficulties with the device architecture. Furthermore, the 
fabrication of thicker films of material would allow for the production of larger lateral flake sizes, resulting in a decreased probability of quenching due to edge effects. This is because there is a general correlation between size and thickness when synthesising liquid exfoliated nanosheets.

To increase the efficiency of emission and the quantum yield from liquid exfoliated monolayer sheets, a large number of techniques could be explored.

Through careful selection of the subphase, the controlled deposition of material via the LangmuirBlodgett method should still be achievable. However, by choosing the subphase, a reduced interaction with the $\mathrm{MoS}_{2}$ (or another material) could be achieved giving an increased photoluminescent signal. Thus, it of interest to explore a number of subphases to determine which results in the least quenching of the optical properties. Dimethyl sulfoxide could also be of interest here.

The underlying substrate that was chosen for deposition, $\mathrm{SiO}_{2}$, was chosen such that the fabricated films could be directly compared to other two-dimensional material systems where $\mathrm{SiO}_{2}$ is the substrate of choice. In principle, the Langmuir-Blodgett method should enable the deposition onto an arbitrary substrate; this can be facilitated by pre-treatment of the substrate in some cases. Thus, the use of alternate substrates such as mica that can magnify the photoluminescence should be explored.

Metal nanoparticles have displayed the ability to enhance the photoluminescence of fluorescent species, with the most commonly used system being Au nanoparticles. With the Langmuir-Blodgett technique, the creation of a double or triple heterostructure of $\mathrm{Au} / \mathrm{MoS}_{2}$ or $\mathrm{Au} / \mathrm{MoS}_{2} / \mathrm{Au}$, respectively, could be straightforwardly accomplished. The plasmonic effect resulting from the presence of these nanoparticles should enhance the emission properties of the $\mathrm{MoS}_{2}$, making measurements of unique maps much simpler. To this end, preliminary experiments of coupled $\mathrm{MoS}_{2} /$ Au nanoparticles systems are underway.

To enhance the quantum yield from monolayer sheets, the deposited material can be treated with specific solutions. Here, the monolayer flakes become functionalised with molecules that can reduce 
the effects of non-radiative recombination. Preliminary experiments have been performed and shown enhancements of deposited films of $\sim 100 x$, as demonstrated in fig 7.2. A redshift in wavelength thought to arise from covalent bonding of molecules to the monolayer sheets has also been observed, but this should only add to the uniqueness, as these molecules should attach in a random manner.

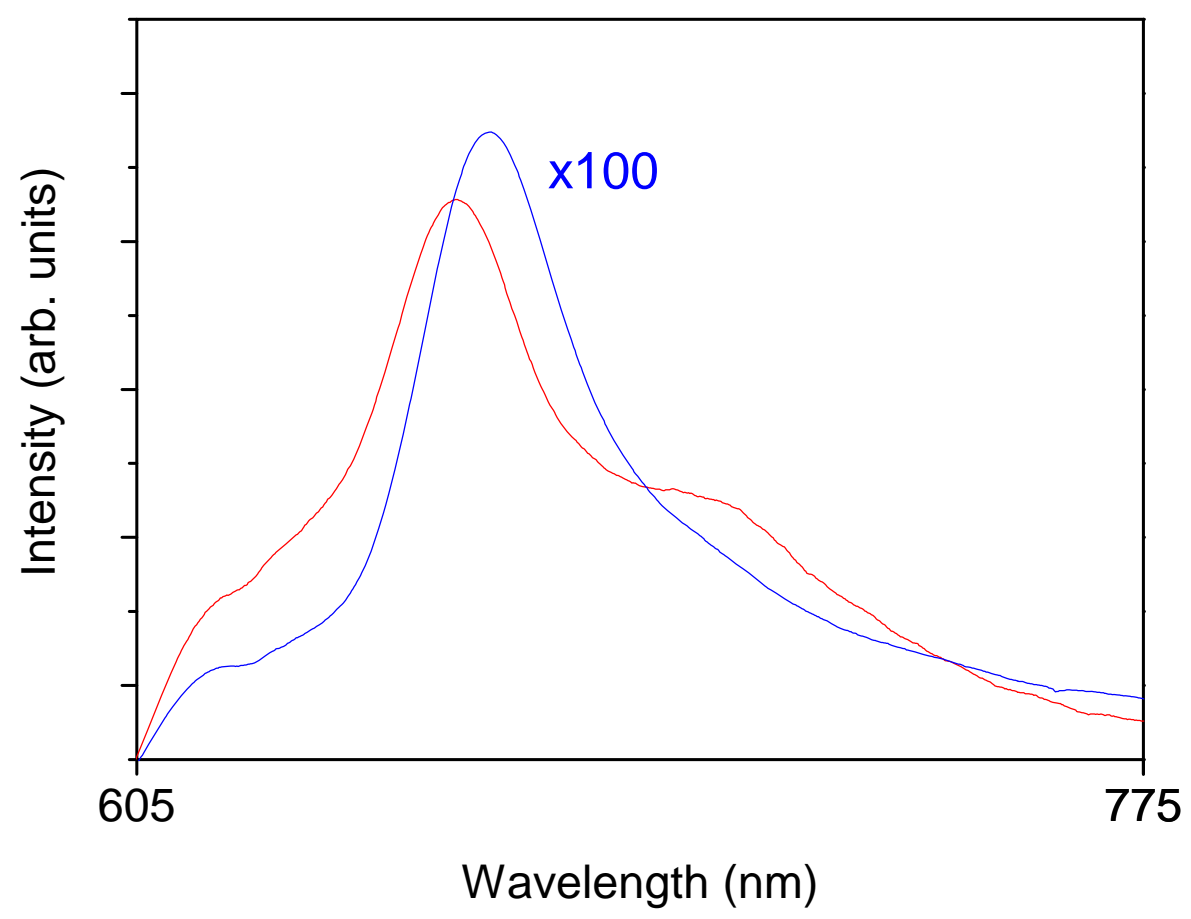

Figure 7.2: The effect of treating a deposited film of $\mathrm{MoS}_{2}$ with a specific solution, known as superacid. The photoluminescent intensity has increased by $\sim 100 x$.

As with the RTDs, the emission from optical tags would need to robust to aging and temperature, so that the fingerprint does not change. The longevity and environmental effects of these devices will therefore have to be explored. Annealing of deposited films can increase the longevity in this regard and also, encapsulation could be employed to protect the thin film. Here, the Langmuir-Blodgett technique could be used to deposit a 2D insulator, such as Boron Nitride, over the thin film. Finally, if the existence of imperfections needs to be enhanced, techniques such as exposure to oxygen plasma can be used to promote the number of defects in the monolayers. 


\section{(c) Graphene/Nanoparticle/Graphene Heterostructures}

The Langmuir-Blodgett technique has been used to create heterostructures capable of linking 0-D and 2-D quantum systems. Furthermore, by altering the subphase to dimethyl sulfoxide, the optical properties of the nanoparticles were shown to remain intact. This has laid the foundations to create an electronically controllable, unique identification device that makes use of an optical measurement.

The subphase, dimethyl sulfoxide (DMSO), has only been employed in a few preliminary results and it should first be experimentally shown that this method of creating closely-packed nanoparticle films that are not quenched, can be repeated.

The fabrication of heterostructures following nanoparticle deposition from the DMSO-air interface has been somewhat elusive. Following the successful transfer of the nanoparticle film and extensive drying, the top graphene contact has showed only minor adhesion, and was completely decoupled from most areas of the film. This is thought to be due to the DMSO being difficult to remove, due to its extremely low vapour pressure. The presence of DMSO (which is hygroscopic) on the surface results in the binding with water upon graphene transfer, this means that the water does not get removed
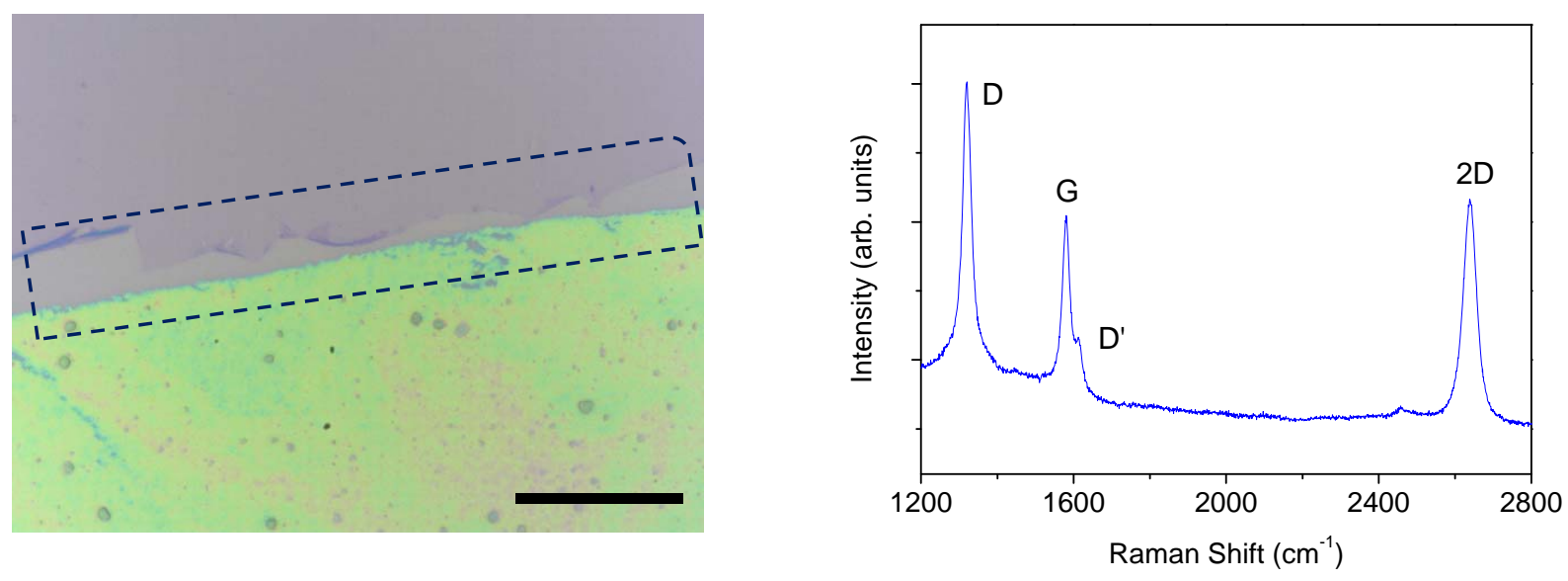

Figure 7.3: Creating heterostructures of nanoparticles following deposition from DMSO. (a) Due to presence of DMSO, graphene layer is decoupled from the nanoparticle film, as highlighted at the film edge where the graphene layer is ripped. Scale bar is $250 \mu \mathrm{m}$. (b) Raman spectroscopy signal of top graphene/NPs. The standard 2D/G peaks are visible whilst the $D^{\prime}$ and $D$ peak indicate a defective nature for the graphene. 
effectively leading to the graphene not being well adhered over the nanoparticles. Preliminary results highlighting this can be seen in fig 7.3a. To circumnavigate this problem, amplified drying in ultra-high vacuum followed by copious rinsing in acetone has been used, and it has been shown that a graphene/nanoparticle/graphene heterostructure has been created. Due to the colour of the nanoparticle film, the graphene was difficult to observe optically, and as such Raman spectroscopy was used to confirm its presence with the preliminary results displayed in fig $7.3 \mathrm{~b}$. The existence of the $G, D$ and 2D peaks are a clear confirmation that graphene is present (where there should only be NPs), although the ratio of their intensities is extremely different to pristine monolayer graphene, indicating a highly defective layer.

The creation of nanoparticle films at the air-water interface displayed a height of approximately 2 layers. By optimising the DMSO deposition, the creation of a single continuous monolayer should be explored. To achieve this, the deposition at a range of surface pressures should be attempted, to optimise the onset of solid film formation. Furthermore, the time between compression of the barriers and deposition of the film should be investigated to observe whether the nanoparticles reorder themselves on the surface if provided adequate time. The dipping rate of the substrate on the formed film morphology should also be examined.

Using heterostructures that are tightly-packed, the conductive-AFM measurements should be remeasured to try and elucidate the mechanisms behind the perplexing conductivity measurements observed in the heterostructure region of the previous samples. Ideally, the close-packing nature of the films deposited from DMSO should prevent any short-circuits and provide an insulating layer that only allows current to pass through tunneling, as expected.

If the creation of improved heterostructures display characteristics that suggest there is no shortcircuits, then individual devices should be defined, isolated and tested. For example, typical devices of $5 \times 5 \mu \mathrm{m}^{2}$ could easily be fabricated for initial tests. The measurement of the I-V characteristics through the devices should be investigated to see if there is any observation of tunneling. 
For creation of unique identities, the defined devices should be electrically contacted so that electroluminescence measurements can be performed. This should be performed at a range of voltages that could potentially act as different challenges in a PUF-like system. Electroluminescence maps of different heterostructures should be compared to investigate the uniqueness of fabricated devices. Interestingly, there is a possibility that the optical spectrum and the I-V characteristic could be combined to form a compound unique signature of the device.

The repeatability of the maps and I-Vs that give the unique identity should also be examined to ensure the system is robust to multiple measurements.

As before, the fabricated devices should be inspected for variations to environmental conditions and aging, for the same reasons. In the case of nanoparticles, over-exposure to light can often result in bleaching of the quantum dot within, which quenches the luminescence. So this additional investigation must be performed for nanoparticles.

Finally, the colloidal synthesis of nanoparticles offers a wide tunability of their band gap. The Langmuir-Blodgett deposition of a range of these nanoparticles should be performed to investigate whether the size and shape have any effect on the deposited films. Different sizes of nanoparticles could also be incorporated into a single device, this would allow efficient emission over a wide wavelength range and should increase the practicality of these devices in unique identification. Also, this could provide a suitable device for broadband solar cells. 